Old Dominion University

ODU Digital Commons

Physics Theses \& Dissertations

Physics

Winter 2005

\title{
Inclusive and Exclusive Compton Processes in Quantum Chromodynamics
}

\author{
A. Psaker \\ Old Dominion University
}

Follow this and additional works at: https://digitalcommons.odu.edu/physics_etds

Part of the Elementary Particles and Fields and String Theory Commons, and the Nuclear Commons

\section{Recommended Citation}

Psaker, A.. "Inclusive and Exclusive Compton Processes in Quantum Chromodynamics" (2005). Doctor of Philosophy (PhD), Dissertation, Physics, Old Dominion University, DOI: 10.25777/ahsy-dv57

https://digitalcommons.odu.edu/physics_etds/72

This Dissertation is brought to you for free and open access by the Physics at ODU Digital Commons. It has been accepted for inclusion in Physics Theses \& Dissertations by an authorized administrator of ODU Digital Commons. For more information, please contact digitalcommons@odu.edu. 


\title{
INCLUSIVE AND EXCLUSIVE COMPTON PROCESSES \\ IN QUANTUM CHROMODYNAMICS
}

by

\author{
A. Psaker \\ B.S. March 1997, University of Ljubljana \\ M.S. May 2001, Old Dominion University
}

A Dissertation Submitted to the Faculty of

Old Dominion University in Partial Fulfillment of the

Requirement for the Degree of

DOCTOR OF PHILOSOPHY

PHYSICS

OLD DOMINION UNIVERSITY

December 2005

Approved by:

Anatolv Radvushkin (Birectdr)

Ian Balitskv

Charles Hyde-Wright

Mark Havey

John Adam 


\title{
ABSTRACT \\ INCLUSIVE AND EXCLUSIVE COMPTON PROCESSES IN QUANTUM CHROMODYNAMICS
}

\author{
A. Psaker \\ Old Dominion University, 2005 \\ Director: Dr. Anatoly Radyushkin
}

In our work, we describe two types of Compton processes. As an example of an inclusive process, we consider the high-energy photoproduction of massive muon pairs off the nucleon. We analyze the process in the framework of the QCD parton model, in which the usual parton distributions emerge as a tool to describe the nucleon in terms of quark and gluonic degrees of freedom. To study its exclusive version, a new class of phenomenological functions is required, namely, generalized parton distributions. They can be considered as a generalization of the usual parton distributions measured in deeply inelastic lepton-nucleon scattering. Generalized parton distributions (GPDs) may be observed in hard exclusive reactions such as deeply virtual Compton scattering. We develop an extension of this particular process into the weak interaction sector. We also investigate a possible application of the GPD formalism to wide-angle real Compton scattering. 
(C)Copyright, 2006, by A. Psaker, All Rights Reserved. 


\section{ACKNOWLEDGMENTS}

I would like to express sincere and deep gratitude to my advisor Anatoly Radyushkin for his generous support and guidance during my graduate student years. I would like to extend my gratitude to all the members of my committee for their helpful comments and suggestions. In particular, I'm grateful to Charles Hyde-Wright and Wally Melnitchouk for various stimulating and valuable discussions. I would like to thank my colleagues at the Physics Department of Old Dominion University and the Theory Group of Jefferson Lab. I'm thankful to my family for their support, and to my wife Mateja for her patience and continuous encouragement. This work was supported by the US Department of Energy DE-FG02-97ER41028 and by the contract DE-AC05-84ER40150 under which the Southeastern Universities Research Association (SURA) operates the Thomas Jefferson Accelerator Facility. 


\section{TABLE OF CONTENTS}

Page

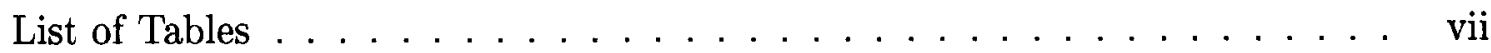

List of Figures $\ldots \ldots \ldots \ldots \ldots \ldots$ viii

\section{CHAPTER}

I Introduction . . . . . . . . . . . . . . . . . 1

II Deeply Inelastic Lepton Scattering . . . . . . . . . . . . . . 5

II.1 Introduction . . . . . . . . . . . . . . . 5

II.2 Kinematics . . . . . . . . . . . . . . . . . 5

II.3 Bjorken Scaling and the Parton Model . . . . . . . . . . . . . . 12

III Phenomenological Functions . . . . . . . . . . . . . . . 17

III.1 Introduction . . . . . . . . . . . . . . . . 17

III.2 Form Factors . . . . . . . . . . . . . . . . . 17

III.3 Parton Distribution Functions . . . . . . . . . . . . . . . . . . 19

III.4 Distribution Amplitudes . . . . . . . . . . . . . . . . . . . 22

III.5 Generalized Parton Distributions . . . . . . . . . . . . . . 23

III.5.1 Skewed Parton Distributions . . . . . . . . . . . . . . . 24

III.5.2 Double Distributions . . . . . . . . . . . . . . . . 29

IV Deeply Virtual Compton Scattering . . . . . . . . . . . . . . . 32

IV.1 Introduction . . . . . . . . . . . . . . . . . . . . 32

IV.2 Virtual Compton Scattering Amplitude . . . . . . . . . . . . . . 33

IV.3 Kinematics . . . . . . . . . . . . . . . . . . . . 41

IV.4 Toy Model . . . . . . . . . . . . . . . . . . . . 48

IV.5 Cross Section . . . . . . . . . . . . . . . 50

V Inclusive Photoproduction of Lepton Pairs . . . . . . . . . . . 57

V.1 Introduction . . . . . . . . . . . . . . . . . 57

V.2 Compton Subprocess . . . . . . . . . . . . . . 58

V.3 Bethe-Heitler Subprocess . . . . . . . . . . . . . . . . 64

V.4 Interference Terms . . . . . . . . . . . . . . . . . . 70

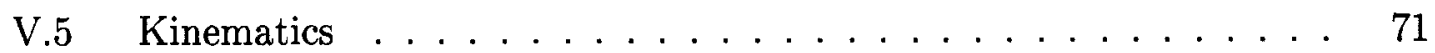

VI Exclusive Photoproduction of Lepton Pairs . . . . . . . . . . . . 75

VI.1 Introduction . . . . . . . . . . . . . . 75

VI.2 Kinematics . . . . . . . . . . . . . . . . . 76

VI.3 Cross Section . . . . . . . . . . . . . . . . 77

VI.3.1 Compton Process . . . . . . . . . . . . . . . . 79

VI.3.2 Bethe-Heitler Process . . . . . . . . . . . . . . . . 83

VII Weak Deeply Virtual Compton Scattering . . . . . . . . . . . . . . 89

VII.1 Introduction . . . . . . . . . . . . . . . . . . . . . . . 89

VII.2 Weak Virtual Compton Scattering Amplitude . . . . . . . . . . 90

VII.2.1 Weak Neutral Amplitude . . . . . . . . . . . . . 91

VII.2.2 Weak Charged Amplitude . . . . . . . . . . . 94 
VII.3 Weak DVCS Processes . . . . . . . . . . . . . . . . . . 96

VII.3.1 Weak Neutral Neutrino-Proton Scattering . . . . . . . . 97

VII.3.2 Weak Neutral Electron-Proton Scattering . . . . . . . . . 99

VII.3.3 Weak Charged Neutrino-Neutron Scattering . . . . . . . . 105

VIII Wide-Angle Real Compton Scattering . . . . . . . . . . . . 111

VIII.1 Introduction . . . . . . . . . . . . . . . . . . . . 111

VIII.2 Twist Decomposition and Parametrization . . . . . . . . . . . 113

VIII.3 Compton Scattering Amplitude . . . . . . . . . . . . . . . . 117

VIII.3.1 Twist-2 Amplitude . . . . . . . . . . . . . . . . . 118

VIII.3.2 Twist-3 Amplitude . . . . . . . . . . . . . . . . . . . . 118

VIII.3.3 Electromagnetic Gauge Invariance . . . . . . . . . . . . . 120

VIII.3.4 Helicity Amplitudes . . . . . . . . . . . . . . . . 121

VIII.3.5 Model . . . . . . . . . . . . . . . . . . . . . . 123

VIII.4 Compton Scattering on a Pion in QED . . . . . . . . . . 130

IX Conclusions and Outlook . . . . . . . . . . . . . . . . . 132

BIBLIOGRAPHY $\ldots \ldots \ldots \ldots \ldots \ldots \ldots \ldots \ldots \ldots \ldots$

\section{APPENDICES}

A Glossary . . . . . . . . . . . . . . . . . . . . 142

B List of Integrals and Scalar Functions That Appear in the Bethe-Heitler Subprocess of the Inclusive Photoproduction of Lepton Pairs . . . . . . 143

C Modified Parametrization of the Nonforward Matrix Element in the Scalar

Toy Model . . . . . . . . . . . . . . . . . . . . 147

$\operatorname{VITA} \ldots \ldots \ldots \ldots \ldots \ldots \ldots \ldots \ldots \ldots \ldots \ldots$ 


\section{LIST OF TABLES}

Page

I Lower and upper limits of the invariant momentum transfer $t$ within the kinematically allowed region illustrated in Fig. 14 for two different lepton beam energies $\omega . \ldots \ldots \ldots$. . . . . . . . . .

II Polar angles $\phi$ and $\phi^{\prime}$ of the incoming and scattered leptons, respectively, in the target rest frame for $Q_{1}^{2}=2.5 \mathrm{GeV}^{2}$ and $x_{B}=0.35$ with two different lepton beam energies $\omega . \ldots . \ldots . . . . .$.

III Polar angles $\phi$ and $\phi^{\prime}$ of the incoming and scattered leptons, respectively, in the target rest frame for $Q_{1}^{2}=2.5 \mathrm{GeV}^{2}$ and $x_{B}=0.35$ with two different lepton beam energies $\omega . \ldots . . . . . . . . .$.

IV Orders of magnitude (in nbarns) for the unpolarized total weak neutral $\sigma_{\nu p}$, Compton weak neutral $\sigma_{C e p}$, Compton weak charged $\sigma_{C \nu n}$ and Compton electromagnetic $\sigma_{C e p}$ cross sections for two different kinematical regions of the Bjorken scaling variable with two different lepton beam energies $\omega . \ldots \ldots$. . . . . . . . . . . . 110 


\section{LIST OF FIGURES}

1 Deeply inelastic electron-nucleon scattering in the one-photon exchange

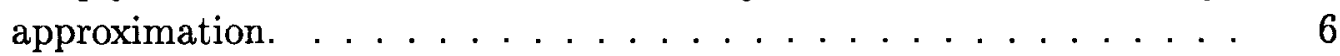

2 Forward virtual Compton scattering amplitude. . . . . . . . . . . 9

3 Kinematics of deeply inelastic electron-nucleon scattering in the parton model. . . . . . . . . . . . . . . . . . .

4 Elastic electron-nucleon scattering in the one-photon exchange approximation. . . . . . . . . . . . . . . . . 20

5 Deeply inelastic electron-nucleon scattering cross section as the imaginary part of the forward virtual Compton scattering amplitude. . . . 22

6 The handbag contribution to the process $\gamma^{*} \gamma \rightarrow \pi^{0} \ldots \ldots \ldots$. . . .

7 Comparison between the descriptions in terms of nonforward and offforward parton distribution functions. . . . . . . . . . . . . .

8 Handbag contribution to the nonforward virtual Compton scattering amplitude. . . . . . . . . . . . . . . . . . 27

9 Description in terms of double distributions. . . . . . . . . . . . 30

10 Conversion from a double distribution to an off-forward parton distribution function with a zero and nonzero skewness. . . . . . . . . .

11 DVCS (a) and Bethe-Heitler (b and c) diagrams contributing to electroproduction of a real photon. . . . . . . . . . . . . . .

12 Handbag diagrams ( $s$ - and $u$-channel) in the virtual Compton scattering amplitude. . . . . . . . . . . . . . . . . 34

13 Kinematics of the generalized DVCS process in the target rest frame.

14 Kinematically allowed region for $\hat{s} \geq 4 \mathrm{GeV}^{2}$ and $Q_{1}^{2} \geq 2.5 \mathrm{GeV}^{2}$ with $\omega=5.75 \mathrm{GeV}$ (solid line) and $\omega=11 \mathrm{GeV}$ (dashed line) lepton beam.

15 Invariant momentum transfer $t$ plotted as a function of the angle $\theta_{\gamma \gamma}$ between the incoming virtual and outgoing real photon in the target rest frame for $Q_{1}^{2}=2.5 \mathrm{GeV}^{2}$ and $x_{B}=0.35 \ldots \ldots \ldots$. . . . .

16 Compton cross section $\sigma_{C}$ plotted as a function of the angle $\theta_{\gamma \gamma}$ between the incoming virtual and outgoing real photon in the target rest frame for $Q_{1}^{2}=2.5 \mathrm{GeV}^{2}$ and $x_{B}=0.35$ with $\omega=5.75 \mathrm{GeV}$ (solid line) and $\omega=11 \mathrm{GeV}$ (dashed line) electron beam. . . . . . . . . . . . . .

17 Bethe-Heitler cross section $\sigma_{B H}$ plotted as a function of the angle $\theta_{\gamma \gamma}$ between the incoming virtual and outgoing real photon in the target rest frame for $Q_{1}^{2}=2.5 \mathrm{GeV}^{2}$ and $x_{B}=0.35$ with $\omega=5.75 \mathrm{GeV}$ (solid line) and $\omega=11 \mathrm{GeV}$ (dashed line) electron beam. . . . . . . . . .

18 Bethe-Heitler cross section $\sigma_{B H}$ plotted as a function of the angle $\theta_{\gamma \gamma}$ between the incoming virtual and outgoing real photon in the target rest frame for $Q_{1}^{2}=2.5 \mathrm{GeV}^{2}$ and $x_{B}=0.35$ with $\omega=5.75 \mathrm{GeV}$ (solid line) and $\omega=11 \mathrm{GeV}$ (dashed line) electron beam. . . . . . . . . 
19 Compton cross section $\sigma_{C}$ (solid line) and Bethe-Heitler cross section $\sigma_{B H}$ (dashed line) plotted as a function of the angle $\theta_{\gamma \gamma}$ between the incoming virtual and outgoing real photon in the target rest frame for $Q_{1}^{2}=2.5 \mathrm{GeV}^{2}$ and $x_{B}=0.35$ with $\omega=5.75 \mathrm{GeV}$ electron beam. . . .

20 Inclusive photoproduction of lepton pairs. . . . . . . . . . . . 55

21 Compton contribution to the inclusive photoproduction of lepton pairs in the parton model. . . . . . . . . . . . . . . . .

22 Bethe-Heitler contribution to the inclusive photoproduction of lepton pairs in the parton model. . . . . . . . . . . . . .

23 Bjorken scaling variable $x_{B}$ plotted as a function of the angle $\theta_{\gamma \gamma}$ between the incoming real and outgoing virtual photon in the target rest frame for $\omega^{\prime}=10 \mathrm{GeV}$ and $M_{\text {pair }}=3 \mathrm{GeV}$ with $\omega=40 \mathrm{GeV}$ photon beam. . . . . . . . . . . . . . . . . .

24 Invariant momentum transfer $t$ plotted as a function of the angle $\theta_{\gamma \gamma}$ between the incoming real and outgoing virtual photon in the target rest frame for $\omega^{\prime}=10 \mathrm{GeV}$ and $M_{\text {pair }}=3 \mathrm{GeV}$ with $\omega=40 \mathrm{GeV}$

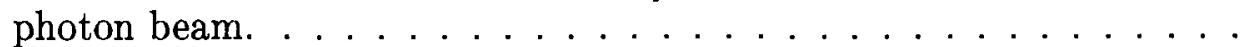
Unpolarized valence quark distributions for $u$-flavor (bold solid line) and $d$-flavor (solid line), and the sea quark distribution (dashed line) in the proton. . . . . . . . . . . . . . . . .

26 Compton cross section $\sigma_{C}$ (solid line) and Bethe-Heitler cross section $\sigma_{B H}$ (dashed line) plotted as a function of the angle $\theta_{\gamma \gamma}$ between the incoming real and outgoing virtual photon in the target rest frame for $\omega^{\prime}=10 \mathrm{GeV}$ and $M_{\text {pair }}=3 \mathrm{GeV}$ with $\omega=40 \mathrm{GeV}$ photon beam. . . .

27 Exclusive photoproduction of lepton pairs. . . . . . . . . . . . .

28 DVCS (a) and Bethe-Heitler (b and c) diagrams contributing to the exclusive photoproduction of a lepton pair. . . . . . . . . . . .

29 Invariant momentum transfer $t$ plotted as a function of the angle $\theta_{\gamma \gamma}$ between the incoming real and outgoing virtual photon in the target rest frame for the invariant mass of the lepton pair $q_{2}^{2}=3 \mathrm{GeV}^{2}$ and $\chi=0.32$ with $\nu_{1}=5 \mathrm{GeV}$ photon beam. . . . . . . . . . . . . .

30 Energy of the scattered nucleon $E_{2}$ plotted as a function of the angle $\theta_{\gamma \gamma}$ between the incoming real and outgoing virtual photon in the target rest frame for the invariant mass of the lepton pair $q_{2}^{2}=3 \mathrm{GeV}^{2}$ and $\chi=0.32$ with $\nu_{1}=5 \mathrm{GeV}$ photon beam. . . . . . . . . . .

31 Contributions from $u$ quarks (solid line) and $d$ quarks (dashed line) to the real part of $\mathcal{H}_{T C S}^{+} \ldots \ldots \ldots \ldots \ldots$. . . . . . . . . .

32 Contributions from $u$ quarks (solid line) and $d$ quarks (dashed line) to

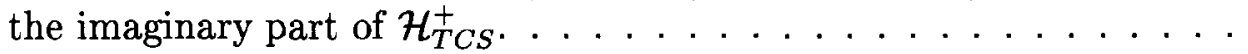

33 Contributions from $u$ quarks (solid line) and $d$ quarks (dashed line) to the real part of $\tilde{\mathcal{H}}_{T C S}^{+}$divided by $g_{A}(t) / g_{A}(t=0)$. . . . . . . . . .

34 Contributions from $u$ quarks (solid line) and $d$ quarks (dashed line) to the imaginary part of $\tilde{\mathcal{H}}_{T C S}^{+}$divided $g_{A}(t) / g_{A}(t=0) \ldots \ldots . .$. 
35 Compton cross section $\sigma_{C}$ plotted as a function of the angle $\theta_{\gamma \gamma}$ between the incoming real and outgoing virtual photon in the target rest frame for $M_{\text {pair }}^{2}=3 \mathrm{GeV}^{2}$ and $\chi=0.32$ with $\nu_{1}=5 \mathrm{GeV}$ photon beam. . . .

36 Bethe-Heitler cross section $\sigma_{B H}$ plotted plotted as a function of the angle $\theta_{\gamma \gamma}$ between the incoming real and outgoing virtual photon in the target rest frame for $M_{\text {pair }}^{2}=3 \mathrm{GeV}^{2}$ and $\chi=0.32$ with $\nu_{1}=5 \mathrm{GeV}$ photon beam. . . . . . . . . . . . . . . . .

37 Compton cross section $\sigma_{C}$ (solid line) and Bethe-Heitler cross section $\sigma_{B H}$ (dashed line) plotted as a function of the angle $\theta_{\gamma \gamma}$ between the incoming real and outgoing virtual photon in the target rest frame for $M_{\text {pair }}^{2}=3 \mathrm{GeV}^{2}$ and $\chi=0.32$ with $\nu_{1}=5 \mathrm{GeV}$ photon beam. . . . .

38 Handbag diagrams ( $s$ - and $u$-channel) in the weak virtual Compton scattering amplitude. . . . . . . . . . . . . . . . . . DVCS (a) and Bethe-Heitler (b and c) diagrams in the weak deeply virtual Compton scattering process. . . . . . . . . . . . . .

40 Weak neutral DVCS cross section $\sigma_{\nu p}$ plotted as a function of the angle $\theta_{B \gamma}$ between the incoming weak virtual boson and outgoing real photon in the target rest frame for $Q_{1}^{2}=2.5 \mathrm{GeV}^{2}$ and $x_{B}=0.35$ with $\omega=5 \mathrm{GeV}$ (solid line) and $\omega=20 \mathrm{GeV}$ (dashed line) neutrino beam.

Compton cross section $\sigma_{C e p}$ for the weak neutral DVCS process plotted as a function of the angle $\theta_{B \gamma}$ between the incoming weak virtual boson and outgoing real photon in the target rest frame for $Q_{1}^{2}=2.5 \mathrm{GeV}^{2}$ and $x_{B}=0.35$ with $\omega=5 \mathrm{GeV}$ (solid line) and $\omega=20 \mathrm{GeV}$ (dashed line) electron beam. . . . . . . . . . . . . . . . . . . . Bethe-Heitler cross section $\sigma_{B H e p}$ for the weak neutral DVCS process plotted as a function of the angle $\theta_{B \gamma}$ between the incoming weak virtual boson and outgoing real photon in the target rest frame for $Q_{1}^{2}=2.5 \mathrm{GeV}^{2}$ and $x_{B}=0.35$ with $\omega=5 \mathrm{GeV}$ (solid line) and $\omega=20 \mathrm{GeV}$ (dashed line) electron beam. . . . . . . . . . . . . . .

43 Bethe-Heitler cross section $\sigma_{B H e p}$ for the weak neutral DVCS process plotted as a function of the angle $\theta_{B \gamma}$ between the incoming weak virtual boson and outgoing real photon in the target rest frame for $Q_{1}^{2}=2.5 \mathrm{GeV}^{2}$ and $x_{B}=0.35$ with $\omega=5 \mathrm{GeV}$ (solid line) and $\omega=20 \mathrm{GeV}$ (dashed line) electron beam. . . . . . . . . . . . . .

44 Compton cross section $\sigma_{C e p}$ (solid line) and Bethe-Heitler cross section $\sigma_{B H e p}$ (dashed line) for the weak neutral DVCS process plotted as a function of the angle $\theta_{B \gamma}$ between the incoming weak virtual boson and outgoing real photon in the target rest frame for $Q_{1}^{2}=2.5 \mathrm{GeV}^{2}$ and $x_{B}=0.35$ with $\omega=5 \mathrm{GeV}$ electron beam. . . . . . . . . . . .

Compton cross section $\sigma_{C \nu n}$ for the weak charged DVCS process plotted as a function of the angle $\theta_{B \gamma}$ between the incoming weak virtual boson and outgoing real photon in the target rest frame for $Q_{1}^{2}=2.5 \mathrm{GeV}^{2}$ and $x_{B}=0.35$ with $\omega=5 \mathrm{GeV}$ (solid line) and $\omega=20 \mathrm{GeV}$ (dashed line) neutrino beam. 
46 Bethe-Heitler cross section $\sigma_{B H \nu n}$ for the weak charged DVCS process plotted as a function of the angle $\theta_{B \gamma}$ between the incoming weak virtual boson and outgoing real photon in the target rest frame for $Q_{1}^{2}=2.5 \mathrm{GeV}^{2}$ and $x_{B}=0.35$ with $\omega=5 \mathrm{GeV}$ (solid line) and $\omega=20 \mathrm{GeV}$ (dashed line) neutrino beam. . . . . . . . . . . . . .

47 Bethe-Heitler cross section $\sigma_{B H \nu n}$ for the weak charged DVCS process plotted as a function of the angle $\theta_{B \gamma}$ between the incoming weak virtual boson and outgoing real photon in the target rest frame for $Q_{1}^{2}=2.5 \mathrm{GeV}^{2}$ and $x_{B}=0.35$ with $\omega=5 \mathrm{GeV}$ (solid line) and $\omega=20 \mathrm{GeV}$ (dashed line) neutrino beam. . . . . . . . . . . . .

48 Compton cross section $\sigma_{C \nu n}$ (solid line) and Bethe-Heitler cross section $\sigma_{B H \nu n}$ (dashed line) for the weak charged DVCS process plotted as a function of the angle $\theta_{B \gamma}$ between the incoming weak virtual boson and outgoing real photon in the target rest frame for $Q_{1}^{2}=2.5 \mathrm{GeV}^{2}$ and $x_{B}=0.35$ with $\omega=5 \mathrm{GeV}$ neutrino beam. . . . . . . . . . . .

49 Handbag contribution ( $a$ and $b$ ) and the configuration (c) with the exchange of two hard gluons. . . . . . . . . . . . . . . . . .

50 Real part of the helicity amplitudes $H_{\text {twist }-2}^{(1,1)}$ (solid line) and $H_{t w i s t-2}^{(2,2)}$ (dashed line) plotted as a function of the center-of-mass scattering angle $\theta_{c m}$ for the invariant $s=5 \mathrm{GeV}^{2} \ldots \ldots \ldots \ldots$

51 Imaginary part of the helicity amplitudes $H_{t w i s t-2}^{(1,1)}$ (solid line) and $H_{\text {twist-2 }}^{(2,2)}$ (dashed line) plotted as a function of the center-of-mass scattering angle $\theta_{c m}$ for the invariant $s=5 \mathrm{GeV}^{2}$. . . . . . . . . . . .

52 Real part of the helicity amplitudes $H_{\text {twist }-2}^{(1,1)}$ (solid line) and $H_{\text {twist-2 }}^{(2,2)}$ (dashed line) plotted as a function of the center-of-mass scattering angle $\theta_{c m}$ for the invariant $s=10 \mathrm{GeV}^{2}$. . . . . . . . . . .

53 Imaginary part of the helicity amplitudes $H_{\text {twist-2 }}^{(1,1)}$ (solid line) and $H_{\text {twist-2 }}^{(2,2)}$ (dashed line) plotted as a function of the center-of-mass scattering angle $\theta_{c m}$ for the invariant $s=10 \mathrm{GeV}^{2} \ldots \ldots \ldots$. . . . . . .

54 Real part of the helicity amplitudes $H_{\text {twist }-2}^{(1,1)}$ (solid line) and $H_{\text {twist-2 }}^{(2,2)}$ (dashed line) plotted as a function of the center-of-mass scattering angle $\theta_{c m}$ for the invariant $s=20 \mathrm{GeV}^{2}$. . . . . . . . . . .

55 Imaginary part of the helicity amplitudes $H_{t w i s t-2}^{(1,1)}$ (solid line) and $H_{\text {twist-2 }}^{(2,2)}$ (dashed line) plotted as a function of the center-of-mass scattering angle $\theta_{c m}$ for the invariant $s=20 \mathrm{GeV}^{2} \ldots \ldots \ldots$. . . . .

56 Twist-2 cross section $\sigma^{(1,1)}$ plotted as a function of the center-of-mass scattering angle $\theta_{c m}$ for the invariant $s=5 \mathrm{GeV}^{2}$ (bold solid line), $s=10 \mathrm{GeV}^{2}$ (solid line) and $s=20 \mathrm{GeV}^{2}$ (dashed line). . . . . . . .

57 Twist-2 cross section $\sigma^{(2,2)}$ plotted as a function of the center-of-mass scattering angle $\theta_{c m}$ for the invariant $s=5 \mathrm{GeV}^{2}$ (bold solid line), $s=10 \mathrm{GeV}^{2}$ (solid line) and $s=20 \mathrm{GeV}^{2}$ (dashed line). . . . . . . .

58 Combination $s^{4.6} d \sigma^{(2,2)} / d t$ plotted as a function of the center-of-mass scattering angle $\theta_{c m}$ for the invariant $s=5 \mathrm{GeV}^{2}$ (bold solid line), $s=10 \mathrm{GeV}^{2}$ (solid line) and $s=20 \mathrm{GeV}^{2}$ (dashed line). . . . . . . 
59 Feynman diagrams for Compton scattering on a pion in QED. . . . 131 


\section{CHAPTER I}

\section{INTRODUCTION}

Strong interaction physics, the study of hadron structure in general, is one of the most fascinating frontiers of modern science. The underlying theory is the universally accepted nonAbelian gauge field theory called quantum chromodynamics (QCD). In principle, QCD embraces all phenomena of hadronic physics. It postulates that hadrons (baryons and mesons) are composite objects made up of quarks, and that the color interaction between quarks is mediated by gluons as the gauge bosons. The main difficulty of this elegant theory lies in the fact that it is formulated in terms of colored degrees of freedom (quarks and gluons) while the physical hadrons are colorless. How the hadrons are built out of quarks and gluons has yet to be answered, and it represents a challenging task.

Historically, QCD originated from the constituent quark model [1, 2]. In the 1960 's, the development of high-energy accelerators made it possible to resolve the structure of hadrons for the first time. The studies of the hadron spectroscopy led to the concept of quarks as the fundamental building blocks of hadrons, in particular, three quarks for baryons and a quark-antiquark pair for mesons. The natural step that followed, was the search for quarks in the experiments with the momentum transfer large enough to look inside the hadrons. The first series of such high-resolution experiments was performed at SLAC by probing the proton structure. The process was known as deeply inelastic electron-proton scattering (DIS). It is probably the most studied QCD process. The data on DIS have strongly supported the parton model, a physical picture given by Feynman $[3,4,5]$, in which the proton, when observed with high spatial resolution, is built out of almost-free and point-like constituents. They were called partons. Thus the dynamics of partons described by the QCD parton model should have the property of asymptotic freedom. In other words, the coupling constant of interaction between partons is vanishing at small distances (or large momentum transfers). In addition, the DIS experiments also suggested that charged partons should be spin-1/2 particles that were later identified with quarks. Another important result from these experiments was the first evidence for the presence of

This dissertation follows the style of The Physical Review. 
electrically neutral gluons. It turns out that quarks carry only about a half of the total nucleon momentum.

The fact that the theory of quark dynamics should exhibit the desired property of the asymptotic freedom led physicists to consider the nonAbelian gauge field theory, originally constructed by Yang and Mills [6], as the best candidate [7, 8, 9]. In order to resolve several difficulties in the constituent quark model, a new quantum number, color, was introduced to quarks $[10,11,12]$. By identifying color as the $\mathrm{SU}(3)$ symmetry of the nonAbelian gauge theory $[13,14]$, QCD was finally established.

The description of all inclusive hard reactions in QCD is possible due to the socalled factorization. According to this property, the cross section of a particular QCD process splits (factorizes) into the hard (short-distance) and the soft (long-distance) parts. Introducing the factorization scale as a point of separation can only be possible by assuring the presence of a large invariant, such as the virtuality of the probe or the momentum transfer $[15,16,17,18,19,20,21,22]$. The clear separation of scales is of the crucial importance. The asymptotically free nature of QCD allows to discuss the short-distance interactions by means of perturbation theory. This is, however, not the case for the soft (nonperturbative) stages of interactions. They are expressed in terms of the hadronic matrix elements of well-defined quark-gluon operators, i.e. the QCD operators taken on the light-cone and sandwiched between the hadronic states. These matrix elements accumulate information about long-distance dynamics. They emerge as a result of the description of the hard hadronic processes using a powerful tool called the light-cone operator product expansion (OPE) [23, 24]. Originally applied to DIS $[25,26,27]$, the technique is based on the statement that, in the particular kinematical regime, the asymptotic behavior of the relevant scattering amplitudes is governed by the singularities on the light-cone which, in the framework of a light-cone expansion, can be described in terms of contributions of definite twist [24] (see [28] for a recent review). In other words, one performs a systematic expansion in the inverse powers of a characteristic momentum scale, and extracts the leading and higher twist contributions, the latter containing information about quark-quark and quark-gluon correlations inside the hadron.

The hadronic matrix elements are process-independent nonperturbative objects measured with the help of different probes, such as photons and weak interaction bosons. For that reason, these objects are parametrized by universal functions, allowing to relate various light-cone dominated scattering processes to each other. The 
well-known example of such phenomenological functions is given by the usual parton distribution functions (PDFs). They parametrize forward hadronic matrix elements and, since these elements are related to inclusive cross sections, PDFs enter the description of hard inclusive reactions. Additional information on quark and gluon structure of hadrons is provided by measuring the nonforward hadronic matrix elements. They correspond to nondiagonal transitions between the hadronic states in momentum, flavor and spin spaces, and hence are described in terms of more general phenomenological functions known as the generalized parton distributions (GPDs) $[29,30,31,32,33,34]$. These distributions can be accessed in hard exclusive reactions [35, 36], such as deeply virtual Compton scattering (DVCS) [30, 31, 32, 33, 34] and deeply exclusive production of mesons (DMP) $[33,34,37]$. In particular, the DVCS process attracted a lot of attention, both theoretical and experimental.

The thesis is organized as follows. In Chapter II, we give a short review on the DIS process and discuss its relation to the forward virtual Compton scattering amplitude (VCA). In addition, we introduce parton distribution functions within the context of the parton model. The commonly used phenomenological functions, such as form factors, PDFs, distribution amplitudes (DAs) and GPDs are the subject of study in Chapter III. We list the definitions and discuss some of theoretical aspects, i.e. the basic properties of these functions and their relations to one another. Typically, GPDs can be measured in hard exclusive leptoproduction processes. DVCS, as the simplest process in this respect, is discussed in Chapter IV. The VCA is calculated at the leading twist within the framework of the nonlocal light-cone expansion of the product of currents in QCD string operators. Moreover, a simple model for the nucleon valence GPDs (i.e. those, which do not include the contribution from sea quarks) is introduced. In Chapter V, we apply the parton model to study the inclusive photoproduction of lepton pairs. Chapter VI is devoted to the exclusive version of the same process. It is related to time-like Compton scattering (TCS), that is the inverse of the DVCS process. With the help of a different set of GPDs, which cannot be accessible in the standard electromagnetic DVCS process, the extension into the weak interaction sector is made possible. This work is presented in Chapter VII. In Chapter VIII, we turn our attention to wide-angle real Compton scattering (WACS). Our approach is based on the handbag dominance, in other words, the light-cone singularities of the Compton scattering amplitude again play an important role $[38,39,40]$. Here we use the formalism of double distributions (DDs) to describe 
nonforward matrix elements of light-cone operators. We study both the leading and next-to-leading twist contributions. Finally, we draw conclusions and outline future research plans in Chapter IX. 


\section{CHAPTER II}

\section{DEEPLY INELASTIC LEPTON SCATTERING}

\section{II.1 INTRODUCTION}

We start with phenomenologically most important of the hard processes, inclusive scattering of high-energy (charged and neutral) leptons on hadrons (to be specific, we will consider here and throughout this thesis, except in Chapter VIII, a spin-1/2 hadronic target, e.g. a nucleon) with the exchange of vector bosons (photons, $W^{ \pm}$ or $\left.Z^{0}\right)$. It played the key role in revealing the quark structure of hadrons, and it is, in addition to electron-positron annihilation into hadrons, the simplest process involving strongly interacting particles. Since leptons do not possess a resolvable internal structure, the reaction cross section depends solely on the internal structure of hadrons.

In Section II.2, we discuss the kinematics and the relation between the hadron tensor and the forward VCA. Both the electron and neutrino-induced DIS cross sections are given in terms of the relevant structure functions. The parton model is introduced in Section II.3. With the help of the parton model master formula, we calculate the DIS cross section, and express the structure functions in terms of PDFs.

\section{II.2 KINEMATICS}

Consider the electron scattering process off the nucleon target through a single photon exchange,

$$
e^{-}(k)+N(P) \longrightarrow e^{-}\left(k^{\prime}\right)+X,
$$

as illustrated in Fig. 1. In other words, we consider only the quantum electrodynamic (or shortly QED) interaction between the electron and the nucleon, and further keep only its lowest order. We denote the nucleon four-momentum with $P$ and the initial and final momenta of electrons with $k$ and $k^{\prime}$, respectively. In the laboratory frame, in which the target is at rest, the nucleon four-momentum is $P=(M, \overrightarrow{0})$, where $M$ stands for the nucleon mass. The electron four-momentum in the initial state is $k=(\omega, \vec{k})$, and in the final state is $k^{\prime}=\left(\omega^{\prime}, \vec{k}^{\prime}\right)$. The momentum transfer to the 


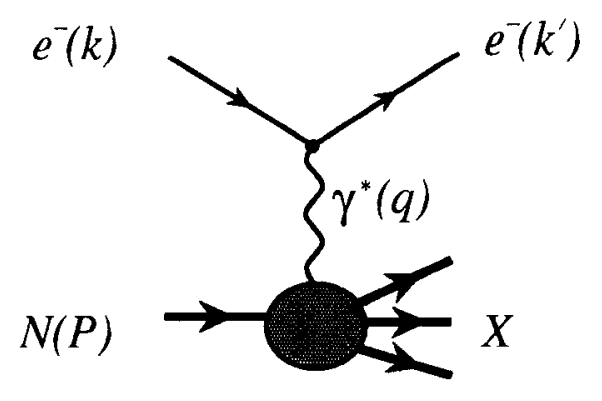

FIG. 1: Deeply inelastic electron-nucleon scattering in the one-photon exchange approximation.

target, carried by the virtual photon, is $q=k-k^{\prime}=(\nu, \vec{q})$. In this scattering process, due to the large $q$, the nucleon breaks up and forms an infinite number of possible hadronic final states labeled by $X$, which remain unobserved. Thus only the outgoing electron is detected. One measures its energy $\omega^{\prime}$ and the scattering angle $\theta$ relative to the incident beam of fixed energy $\omega$.

There are two characteristic Lorentz-invariant quantities of the process, namely, the virtuality of the photon (since it is space-like, $q^{2}<0$, one conventionally defines a positive quantity),

$$
q^{2} \equiv-Q^{2}
$$

which fixes the spatial resolution of the scattering process, $\lambda \sim 1 / \sqrt{Q^{2}}$, and the invariant mass of the final hadronic state,

$$
W^{2} \equiv(P+q)^{2}=M^{2}+2(P \cdot q)-Q^{2} .
$$

In the laboratory frame, they are given by

$$
Q^{2}=4 \omega \omega^{\prime} \sin ^{2}(\theta / 2)
$$

and

$$
W^{2}=M^{2}+2 M \nu-Q^{2} .
$$

Note that in Eq. (4) we have neglected the electron mass compared to its energy. Unlike elastic scattering, for which $W^{2}=M^{2}$ and the momentum transfer squared $Q^{2}$ is connected by the relation $\nu=Q^{2} / 2 M$ to the energy loss of the electron $\nu$, we 
now have two independent variables, $\nu$ and $Q^{2}$. The inclusive differential cross section (see, e.g. $[5,41,42,43]$ ) factors into the electron tensor $L_{\mu \nu}^{(e)}$ and the hadron tensor $W^{\mu \nu}$,

$$
\frac{d^{2} \sigma_{(e N)}}{d \Omega d \omega^{\prime}}=\frac{\alpha^{2}}{Q^{4}} \frac{\omega^{\prime}}{\omega} L_{\mu \nu}^{(e)} W^{\mu \nu},
$$

where $d \Omega$ is the solid angle into which the electron is scattered and $\alpha=e^{2} / 4 \pi \simeq$ $1 / 137$ is the electromagnetic fine structure constant (in the following we use the Heaviside-Lorentz convention together with the natural choice of units, frequently used in particle physics, in which $\hbar=c=1$ ). The electron tensor can be computed exactly in QED. For unpolarized electron scattering, the expression for the spinaveraged electron tensor is

$$
\begin{aligned}
L_{\mu \nu}^{(e)} & =\frac{1}{2} \sum_{s, s^{\prime}} \bar{u}(k, s) \gamma_{\mu} u\left(k^{\prime}, s^{\prime}\right) \bar{u}\left(k^{\prime}, s^{\prime}\right) \gamma_{\nu} u(k, s) \\
& =2\left[k_{\mu} k_{\nu}^{\prime}+k_{\nu} k_{\mu}^{\prime}-g_{\mu \nu}\left(k \cdot k^{\prime}\right)\right] .
\end{aligned}
$$

The hadron tensor, on the other hand, describes the response of the nucleon and therefore, it should include all possible transitions of the nucleon from its ground state $|N(P, S)\rangle$ to any hadronic final state $\left|X\left(P^{\prime}\right)\right\rangle$. It is worth noting that at present, the nonperturbative nature of strong interactions prevents us from calculating the hadron tensor directly within the framework of QCD. Formally, it can be written as

$$
\begin{aligned}
W^{\mu \nu}= & \frac{1}{4 \pi M} \sum_{X}(2 \pi)^{4} \delta^{(4)}\left(P^{\prime}-P-q\right) \frac{1}{2} \sum_{S}\left\langle N(P, S)\left|J_{E M}^{\mu}(0)\right| X\left(P^{\prime}\right)\right\rangle \\
& \times\left\langle X\left(P^{\prime}\right)\left|J_{E M}^{\nu}(0)\right| N(P, S)\right\rangle
\end{aligned}
$$

where $J_{E M}^{\mu}$ is the quark electromagnetic current. Using the translation invariance,

$$
\left\langle N(P, S)\left|J_{E M}^{\mu}(z)\right| X\left(P^{\prime}\right)\right\rangle=\left\langle N(P, S)\left|J_{E M}^{\mu}(0)\right| X\left(P^{\prime}\right)\right\rangle e^{-i\left(P^{\prime}-P\right) \cdot z},
$$

and the completeness condition of the states $\left|X\left(P^{\prime}\right)\right\rangle$,

$$
\sum_{X}\left|X\left(P^{\prime}\right)\right\rangle\left\langle X\left(P^{\prime}\right)\right|=\mathbf{1}
$$

the hadron tensor can be presented in a more compact form, namely,

$$
W^{\mu \nu}=\frac{1}{4 \pi M} \int d^{4} z e^{i(q \cdot z)} \frac{1}{2} \sum_{S}\left\langle N(P, S)\left|J_{E M}^{\mu}(z) J_{E M}^{\nu}(0)\right| N(P, S)\right\rangle .
$$

Adopting a simplifying notation,

$$
\langle N(P)|\mathcal{O}| N(P)\rangle \equiv \frac{1}{2} \sum_{S}\langle N(P, S)|\mathcal{O}| N(P, S)\rangle
$$


where $\mathcal{O}$ is an arbitrary operator we have

$$
W^{\mu \nu}=\frac{1}{4 \pi M} \int d^{4} z e^{i(q \cdot z)}\left\langle N(P)\left|J_{E M}^{\mu}(z) J_{E M}^{\nu}(0)\right| N(P)\right\rangle .
$$

Interchanging the currents inside the matrix element leads to a delta function $\delta^{(4)}\left(P^{\prime}-P+q\right)$ which, in the laboratory frame, requires that $E^{\prime}=M-\nu<M$. Note that such a state $\left|X\left(P^{\prime}\right)\right\rangle$ does not exist since the nucleon $|N(P, S)\rangle$ is the ground state baryon. Hence the delta function cannot be satisfied and the expression with the interchanged currents vanishes. By adding this vanishing matrix element, one can also rewrite the hadron tensor as a current commutator,

$$
W^{\mu \nu}=\frac{1}{4 \pi M} \int d^{4} z e^{i(q \cdot z)}\left\langle N(P)\left|\left[J_{E M}^{\mu}(z), J_{E M}^{\nu}(0)\right]\right| N(P)\right\rangle .
$$

Next we discuss the relation between $W^{\mu \nu}$ and the object known as the forward virtual Compton scattering amplitude (VCA). The latter, shown in Fig. 2, is defined as a Fourier transform of the correlation function of two electromagnetic currents,

$$
T^{\mu \nu}=i \int d^{4} z e^{i(q \cdot z)}\left\langle N(P, S)\left|T\left\{J_{E M}^{\mu}(z) J_{E M}^{\nu}(0)\right\}\right| N(P, S)\right\rangle .
$$

The time-ordering symbol $T$ inside the matrix element instructs us to place the operators into chronological order with the operator having the later time argument to the left. The amplitude (15) is averaged over the nucleon spin. It corresponds to the scattering amplitude for the Compton process off the proton in the forward direction, when contracted with the polarization vectors of the off-shell photon of momentum $q, q^{2} \neq 0$

$$
i \mathrm{~T}(\gamma p \rightarrow \gamma p)=(-i|e|)^{2} \epsilon_{\mu}^{*}(q) \epsilon_{\nu}(q)\left(-i T^{\mu \nu}\right),
$$

or

$$
\mathrm{T}(\gamma p \rightarrow \gamma p)=e^{2} \epsilon_{\mu}^{*}(q) \epsilon_{\nu}(q) T^{\mu \nu} .
$$

Here $e$ denotes the electric charge of the electron. With the help of the optical theorem, the hadron tensor can be written as

$$
W^{\mu \nu}=\frac{1}{2 \pi M} \Im T^{\mu \nu}
$$

Lastly, it is customary to express the hadron tensor, see Eqs. (13) and (14), in terms of the so-called structure functions. The tensor expression is Lorentz-invariant, 


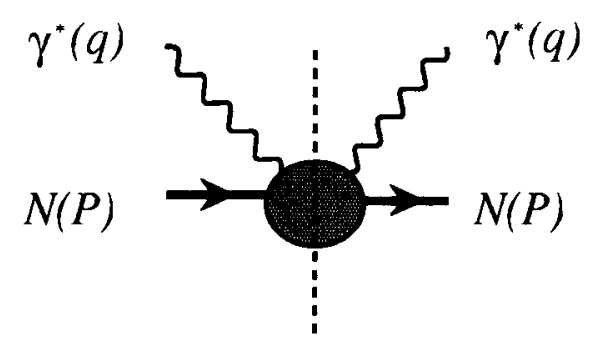

FIG. 2: Forward virtual Compton scattering amplitude. The dashed line denotes the cut, which gives the imaginary part, and it is further related to the hadron tensor through the optical theorem.

and it should also be invariant under parity and time reversal. Together with the electromagnetic current conservation, $\partial_{\mu} J_{E M}^{\mu}=0$, implying the electromagnetic gauge invariance,

$$
q_{\mu} W^{\mu \nu}=0 \text { and } W^{\mu \nu} q_{\nu}=0
$$

one arrives at the most general form for $W^{\mu \nu}$ in the case of unpolarized scattering. It is determined only by two independent scalar structure functions (or alternatively the response functions),

$$
\begin{aligned}
W^{\mu \nu}= & W_{1}\left(\nu, Q^{2}\right)\left[-g^{\mu \nu}+\frac{q^{\mu} q^{\nu}}{q^{2}}\right] \\
& +\frac{W_{2}\left(\nu, Q^{2}\right)}{M^{2}}\left[P^{\mu}-q^{\mu} \frac{(P \cdot q)}{q^{2}}\right]\left[P^{\nu}-q^{\nu} \frac{(P \cdot q)}{q^{2}}\right] .
\end{aligned}
$$

The hadron tensor (20) is symmetric with respect to Lorentz indices $\mu$ and $\nu$. In the case of polarized scattering the tensor has an extra term, which is antisymmetric with respect to $\mu$ and $\nu$ and contains two additional structure functions. We notice that both functions $W_{1}$ and $W_{2}$ depend on two invariant variables, $\nu=(P \cdot q) / M$ and $Q^{2}$. Substituting Eqs. (7) and (20) into Eq. (6) and using Eq. (4), gives the differential cross section in the laboratory frame in the form

$$
\frac{d^{2} \sigma_{(e N)}}{d \Omega d \omega^{\prime}}=\frac{\alpha^{2}}{4 \omega^{2} \sin ^{4}(\theta / 2)}\left[2 W_{1}\left(\nu, Q^{2}\right) \sin ^{2}(\theta / 2)+W_{2}\left(\nu, Q^{2}\right) \cos ^{2}(\theta / 2)\right] .
$$

Usually, $\nu$ is replaced by another Lorentz-invariant quantity, the Bjorken scaling 
variable,

$$
x_{B} \equiv \frac{Q^{2}}{2(P \cdot q)},
$$

and further the dimensionfull $W$-structure functions by their dimensionless partners defined as

$$
\begin{aligned}
& F_{1}\left(x_{B}, Q^{2}\right) \equiv M W_{1}\left(\nu, Q^{2}\right) \\
& F_{2}\left(x_{B}, Q^{2}\right) \equiv \nu W_{2}\left(\nu, Q^{2}\right) .
\end{aligned}
$$

Since the invariant mass of the unobserved hadronic final state is always larger than the nucleon mass, it follows from Eqs. (3) and (22) that

$$
x_{B}=\frac{Q^{2}}{W^{2}-M^{2}+Q^{2}}=\frac{1}{1+\left(W^{2}-M^{2}\right) / Q^{2}} \in(0,1] .
$$

In particular, the kinematical point $x_{B}=1$ corresponds to elastic electron-nucleon scattering. In addition to $x_{B}$, there is another dimensionless variable frequently used, the so-called inelasticity parameter,

$$
y \equiv \frac{(P \cdot q)}{(P \cdot k)}
$$

It specifies, in the laboratory frame, the fraction of the electron energy that is transferred to the nucleon, $y=1-\omega^{\prime} / \omega$, and hence it is also kinematically constrained to the region $(0,1]$. The invariants $Q^{2}, x_{B}$ and $y$ are related to each other through

$$
Q^{2}=x_{B} y\left(s-M^{2}\right) \text {, }
$$

where $s \equiv(k+P)^{2}$ is the usual Mandelstam invariant of the scattering process. The last formula is obtained by writing the Bjorken scaling variable in the laboratory frame, $x_{B}=Q^{2} / 2 M \nu$. Sometimes it is convenient to present the cross section in the invariant form. After some algebra, the cross section results, in terms of dimensionless structure functions, into

$$
\begin{aligned}
\frac{d^{2} \sigma_{(e N)}}{d x_{B} d y} & =\left(\frac{2 \pi M \omega y}{\omega^{\prime}}\right) \frac{d^{2} \sigma_{(e N)}}{d \Omega d \omega^{\prime}} \\
& =\frac{4 \pi \alpha^{2}}{Q^{2} y}\left[y^{2} F_{1}\left(x_{B}, Q^{2}\right)+\left(\frac{1-y}{x_{B}}-\frac{M^{2} y}{s-M^{2}}\right) F_{2}\left(x_{B}, Q^{2}\right)\right]
\end{aligned}
$$

For the sake of completeness, let us investigate the weak version of inclusive leptonnucleon scattering. In general, the weak boson is used as a probe to study the nucleon 
structure. To be specific, we shall consider only the charged current case using muon neutrinos. The latter convert into muons by the emission of a $W^{+}$boson. The reaction is

$$
\nu_{\mu}(k)+N(P) \longrightarrow \mu^{-}\left(k^{\prime}\right)+X
$$

Recall that the incident neutrino is always left-handed and for that reason, there is no averaging over the initial spin. Moreover, having the initial lepton (i.e. neutrino) state polarized yields an extra term in the lepton tensor that is antisymmetric in Lorentz indices $\mu$ and $\nu$,

$$
L_{\mu \nu}^{(\nu)}=8\left[k_{\mu} k_{\nu}^{\prime}+k_{\nu} k_{\mu}^{\prime}-g_{\mu \nu}\left(k \cdot k^{\prime}\right)+i \epsilon_{\mu \nu \sigma \tau} k^{\sigma} k^{\prime \tau}\right]
$$

In constructing the corresponding hadron tensor we have to respect that the parity in the weak interaction is no longer conserved. Thus, due to the presence of the parityviolating term $i \epsilon^{\mu \nu \alpha \beta} P_{\alpha} q_{\beta}$ in the hadron tensor, a third response function $W_{3}\left(\nu, Q^{2}\right)$ arises in the expression for the cross section. In analogy with Eq. (6), along with the factor replacement $\alpha / Q^{2} \rightarrow G_{F} / 4 \pi \sqrt{2}$, where $G_{F} \equiv g^{2} / 4 \sqrt{2} M_{W}^{2} \simeq 1.166 \cdot 10^{-5} \mathrm{GeV}^{-2}$ is the so-called Fermi constant with $g$ being the weak interaction constant and $M_{W}$ the mass of the $W$-boson (note that one chooses the coupling constant of the $W$ boson to the nucleon as $g / 2 \sqrt{2}$, and that the factor $1 / M_{W}^{2}$ in the definition of $G_{F}$ comes from the $W$-boson propagator considered in the limit $Q^{2} \ll M_{W}^{2}$, in other words, one deals with the Fermi contact interaction), the laboratory differential cross section is

$$
\begin{aligned}
\frac{d^{2} \sigma_{(\nu N)}}{d \Omega d \omega^{\prime}}= & \frac{G_{F}^{2} \omega^{\prime 2}}{2 \pi^{2}}\left[2 W_{1}^{(\nu N)}\left(\nu, Q^{2}\right) \sin ^{2}(\theta / 2)+W_{2}^{(\nu N)}\left(\nu, Q^{2}\right) \cos ^{2}(\theta / 2)\right. \\
& \left.+\frac{\omega+\omega^{\prime}}{2 M} W_{3}^{(\nu N)}\left(\nu, Q^{2}\right) \sin ^{2}(\theta / 2)\right] .
\end{aligned}
$$

Finally, in terms of the Lorentz invariant variables $x_{B}$ and $y$, the invariant cross section reads

$$
\begin{aligned}
\frac{d^{2} \sigma_{(\nu N)}}{d x_{B} d y}= & \frac{G_{F}^{2}\left(s-M^{2}\right)}{2 \pi}\left[x_{B} y^{2} F_{1}^{(\nu N)}\left(x_{B}, Q^{2}\right)+(1-y) F_{2}^{(\nu N)}\left(x_{B}, Q^{2}\right)\right. \\
& \left.+y\left(1-\frac{y}{2}\right) F_{3}^{(\nu N)}\right] .
\end{aligned}
$$

Similarly to the electromagnetic case, the dimensionless structure functions in the weak interaction sector are defined as follows

$$
F_{1}^{(\nu N)}\left(x_{B}, Q^{2}\right) \equiv M W_{1}^{(\nu N)}\left(\nu, Q^{2}\right)
$$




$$
\begin{aligned}
& F_{2}^{(\nu N)}\left(x_{B}, Q^{2}\right) \equiv \nu W_{2}^{(\nu N)}\left(\nu, Q^{2}\right) \\
& F_{3}^{(\nu N)}\left(x_{B}, Q^{2}\right) \equiv \nu W_{3}^{(\nu N)}\left(\nu, Q^{2}\right)
\end{aligned}
$$

\section{II.3 BJORKEN SCALING AND THE PARTON MODEL}

The nucleon structure with the size of the order $\lambda<0.2 \mathrm{fm}$ can only be resolved at sufficiently large $Q^{2} \geq 1 \mathrm{GeV}^{2}$. Having large $Q^{2}$ also implies large values of $\nu$, more precisely $\nu^{2} \gg Q^{2}$, since the ratio $x_{B}=Q^{2} / 2 M \nu$ is finite and bounded between 0 and 1. Accordingly, a massive hadronic state $X$ is produced with the invariant mass equal to

$$
W^{2}=Q^{2}\left(1-x_{B}\right) / x_{B}+M^{2} \gg M^{2}
$$

It lies well above the resonance region, $W \geq 2 \mathrm{GeV}$. In this kinematical regime, known as the deeply inelastic region, the structure functions $F\left(x_{B}, Q^{2}\right)$ extracted from the measured inelastic cross sections do not depend significantly on $Q^{2}$ but rather only on $x_{B}$. This interesting feature is termed Bjorken scaling [44, 45, 46, 47]. It can be stated in the following way: in the Bjorken limit, where both $Q^{2} \rightarrow \infty$ and $\nu \rightarrow \infty$ whereas $x_{B}$ is kept fixed, one experimentally observes

$$
F\left(x_{B}, Q^{2}\right) \longrightarrow F\left(x_{B}\right)
$$

The fact that, for sufficiently large values of $Q^{2}$, the structure functions are independent of $Q^{2}$ implies that the nucleon, or any other hadron, is made of point-like constituents. Recall that a finite size object must have a form factor and hence introduce some dependence on $Q^{2}$. Furthermore, since the structure functions are Lorentz invariant, one can study the scattering process in any reference frame. However, the description is considerably simplified, if we look at the nucleon in a very fast moving system with its momentum approaching to infinity along the $z$-direction, i.e. in the so-called infinite momentum frame (the physics of the process is, of course, independent of this choice). Then the transverse momenta, the rest masses of the nucleon constituents and, for consistency, the nucleon mass can be neglected and accordingly, the nucleon momentum is $P^{\mu}=\left(P_{z}, \overrightarrow{0}_{\perp}, P_{z}\right)$. In other words, the structure of the nucleon is described only in terms of the longitudinal momenta of its constituents. This is the basis of the parton model of Feynman [4], which gives the clearest physical interpretation of scaling. In the parton picture, the nucleon is viewed as a collection of noninteracting, point-like constituents, the partons. The interaction of the electron 
with the nucleon can be then viewed as the incoherent sum of interactions between the electron and the individual partons. This approximation is valid as long as the duration of the electron-parton interaction, which is regarded as elastic scattering, is so short that the interaction between the partons themselves can be safely neglected. Thus we consider the scattering process in the impulse approximation by picking up only the lowest-order electromagnetic contribution, and neglect all the QCD corrections associated with the exchange or emission of gluons.

In the infinite momentum frame, a given parton is characterized by the longitudinal momentum fraction $x \in[0,1]$ of the total nucleon momentum $P$,

$$
p=x P
$$

In addition, for each parton species $a$, we define the parton distribution function (PDF) $f_{a / N}(x)$. It describes the probability of finding a parton of type $a$ at the longitudinal momentum fraction $x$ inside the target nucleon $N$. Note that these functions cannot be computed using QCD perturbation theory. All fractions $x$ have to add up to 1 and hence the normalization

$$
\sum_{a} \int_{0}^{1} d x x f_{a / N}(x)=1
$$

holds. The scattered parton has the momentum $p^{\prime}=p+q$, given by the fourmomentum conservation, where $q$ is the momentum of the virtual photon. Since the parton is on its mass shell, it follows that

$$
\begin{aligned}
p^{2} & =(x P+q)^{2} \\
& =x^{2} M^{2}+2 x(P \cdot q)-Q^{2} \\
& \approx 0 .
\end{aligned}
$$

Neglecting $x^{2} M^{2}$ compared to $Q^{2}$ and $\nu$ (recall that $(P \cdot q)=M \nu$ ) yields

$$
x=\frac{Q^{2}}{2(P \cdot q)} \equiv x_{B} .
$$

The longitudinal momentum fraction is found to be identical to the Bjorken scaling variable. It means that the parton must have the fraction $x_{B}$ of the nucleon momentum in order to absorb the virtual photon. It should be emphasized, however, that the variable $x_{B}$ has this simple meaning only in the infinite momentum frame.

Due to the fact that the partons are point-like and noninteracting, the DIS cross section for nucleon-electron scattering in the parton picture is simply given by the 


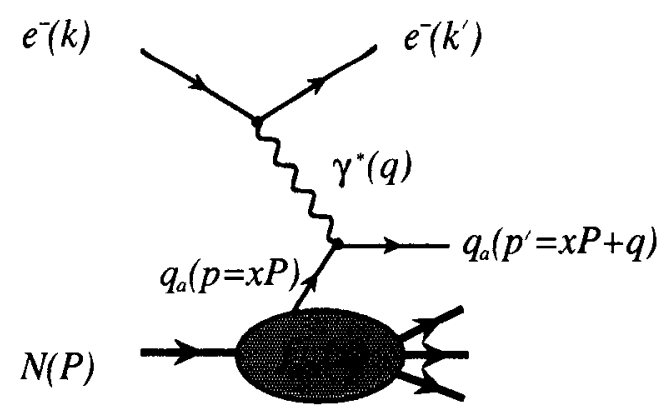

FIG. 3: Kinematics of deeply inelastic electron-nucleon scattering in the parton model.

incoherent sum over all the contributing partons (i.e. the partonic cross sections for elastic scattering of an electron from the individual partons), weighted with the proper distribution functions. The fundamental relation, known as the parton model master formula, reads

$$
\begin{aligned}
\sigma\left[e^{-}(k) N(P) \rightarrow e^{-}\left(k^{\prime}\right) X\right]= & \sum_{a} \int_{0}^{1} d x f_{a / N}(x) \\
& \times \sigma\left[e^{-}(k) q_{a}(x P) \rightarrow e^{-}\left(k^{\prime}\right) q_{a}\left(p^{\prime}\right)\right],
\end{aligned}
$$

where the sum runs over quarks and antiquarks, in order words, over all charged partons because they are the ones that interact with the virtual photon. Schematically, see Fig. 3, the hadronic interaction is broken into a PDF part, represented by the blob, and hard (parton-electron) scattering part. According to the factorization, PDFs do not interfere with the hard scattering part. For that reason, they are universal in a sense that they are same for all inclusive hard scattering processes, not only for electromagnetic DIS [48].

Let us work out the explicit leading-order formula for the DIS cross section using Eq. (39). First we calculate, at the most elementary level, the cross section for electron scattering from a single parton. For $M^{2} \ll-u, s, Q^{2}$, where $u \equiv\left(k^{\prime}-P\right)^{2}$ is the Mandelstam variable, one has

$$
Q^{2}=x_{B} y s \text { and } y=\frac{s+u}{s},
$$

and the invariant form of the partonic double differential cross section is (see, e.g. $[41,49])$

$$
\frac{d^{2} \sigma\left(e^{-} q_{a} \rightarrow e^{-} q_{a}\right)}{d x_{B} d y}=\frac{2 \pi \alpha^{2} Q_{a}^{2} s}{Q^{4}}\left[1+(1-y)^{2}\right] x_{B} \delta\left(x-x_{B}\right)
$$


The electric charge of the parton of type $a$ in units of $|e|$ is denoted by $Q_{a}$. Substituting Eq. (41) into Eq. (39) results in

$$
\frac{d^{2} \sigma\left(e^{-} N \rightarrow e^{-} X\right)}{d x_{B} d y}=\frac{2 \pi \alpha^{2} s}{Q^{4}}\left[1+(1-y)^{2}\right] \sum_{a} Q_{a}^{2} x_{B} f_{a / N}\left(x_{B}\right) .
$$

Comparing now Eqs. (42) and (27), under the assumption that $M^{2} \ll s$, gives

$$
2\left[x_{B} y^{2} F_{1}\left(x_{B}, Q^{2}\right)+(1-y) F_{2}\left(x_{B}, Q^{2}\right)\right]=\left[1+(1-y)^{2}\right] \sum_{a} Q_{a}^{2} x_{B} f_{a / N}\left(x_{B}\right) .
$$

We notice that the right-hand side of Eq. (43) depends only on $x_{B}$ and $y$. Therefore,

$$
x_{B} y^{2} F_{1}\left(x_{B}, Q^{2}\right)+(1-y) F_{2}\left(x_{B}, Q^{2}\right)=\text { const. for all } Q^{2}
$$

or,

$$
\begin{aligned}
& F_{1}\left(x_{B}, Q^{2}\right)=F_{1}\left(x_{B}\right), \\
& F_{2}\left(x_{B}, Q^{2}\right)=F_{2}\left(x_{B}\right) .
\end{aligned}
$$

Hence the structure functions in the parton model exhibit the scaling property, i.e. they do not depend on $Q^{2}$. Conversely, Bjorken scaling suggests the asymptotically free quark dynamics, the property that the quark interaction gets weaker at short distances. In addition, by comparing powers of $y$ in Eq. (43), we can express the $F$-structure functions in terms of PDFs, namely,

$$
\begin{aligned}
& F_{1}\left(x_{B}\right)=\frac{1}{2} \sum_{a} Q_{a}^{2} f_{a / N}\left(x_{B}\right), \\
& F_{2}\left(x_{B}\right)=\sum_{a} Q_{a}^{2} x_{B} f_{a / N}\left(x_{B}\right) .
\end{aligned}
$$

Finally, from Eq. (46) one can read off the following simple relation specific to the scattering of electrons from massless fermions

$$
F_{2}\left(x_{B}\right)=2 x_{B} F_{1}\left(x_{B}\right) .
$$

It is known as the Callan-Gross relation [50], and it is an important evidence that the partons detected in DIS are indeed the spin-1/2 quarks of hadron spectroscopy [48]. It is worth noting that a more descriptive notation is commonly used, in which $f_{a / N}(x)$ is replaced by the flavor labels $u_{N}(x), d_{N}(x), s_{N}(x), \bar{u}_{N}(x)$, etc. Then the proton structure function $F_{1}$ is

$$
F_{1 p}(x)=\frac{1}{2}\left\{\frac{4}{9}\left[u_{p}(x)+\bar{u}_{p}(x)\right]+\frac{1}{9}\left[d_{p}(x)+\bar{d}_{p}(x)\right]+\frac{1}{9}\left[s_{p}(x)+\bar{s}_{p}(x)\right]\right\}
$$


and similarly for $F_{2 p}(x)$.

In summary, the parton model serves both to explain scaling and to identify partons as quarks. However, it is important to add that ultimately, the success of this simple model must be justified in quantum field theory, in particular within the framework of the QCD operator product expansion (OPE). It turns out that, in the Bjorken regime, the dominant contribution to $W^{\mu \nu}$, see Eq. (13), comes from the integration region $0 \leq z^{2} \leq$ const. $/ Q^{2}$. Accordingly, the DIS cross section is dominated by the light-cone region, $z^{2} \rightarrow 0$, of the space-time integration in Eq. (13) [51]. In fact, the scattering amplitudes of other hard inclusive and exclusive processes are also governed by the product of currents $J^{\mu}(z) J^{\nu}(0)$ near the light-cone. Thus OPE is a powerful tool which, not only recovers the parton model predictions but also allows to analyze, in a systematic way, the terms in the light-cone expansion contributing to a given power of $1 / Q^{2}$. 


\section{CHAPTER III}

\section{PHENOMENOLOGICAL FUNCTIONS}

\section{III.1 INTRODUCTION}

The fundamental particles, from which hadrons are built, are known. They are quarks and gluons. The interactions between them are described by the QCD Lagrangian, which is also established. Unfortunately, knowing the first principles is not sufficient. We still need to understand how QCD works, in other words, how to translate the information obtained from experiments on the hadronic level into the language of quark and gluon fields. One may, for example, consider projecting these fields onto hadronic states. The resulting matrix elements can be interpreted as hadronic wave functions $[52,53]$. An alternative approach to describe the hadronic structure is to use different phenomenological functions. The well-known examples are form factors, usual parton distributions functions and distribution amplitudes. Since they have been around for a long time, they are termed the old phenomenological functions. We discuss them separately in Sections III.2, III.3 and III.4. On the other hand, the concept of generalized parton distributions [29, 30, 31, 32, 33, 34] (for reviews, see $[54,36,55]$ and recently [56]) is new. These new phenomenological functions are hybrids of the old ones and therefore, provide a unified and more detailed description of the hadronic structure. Different species of generalized parton distributions are presented in Section III.5 together with some of their general properties.

\section{III.2 FORM FACTORS}

Form factors are defined through the matrix elements of electromagnetic and weak (neutral and charged) currents between the hadronic states. In particular, the matrix element of the electromagnetic current (for the weak currents, see Section VII.3),

$$
J_{E M}^{\mu}(0)=\sum_{f} Q_{f} \bar{\psi}_{f}(0) \gamma^{\mu} \psi_{f}(0)
$$

where $Q_{f}$ is the electric charge (in units of $|e|$ ) of the quark of flavor $f$, between the nucleon states $N\left(p_{1}, s_{1}\right)$ and $N\left(p_{2}, s_{2}\right)$ is parametrized in terms of two independent 
nucleon electromagnetic form factors known as the Dirac and Pauli form factors. Namely,

$$
\begin{aligned}
\left\langle N\left(p_{2}, s_{2}\right)\left|J_{E M}^{\mu}(0)\right| N\left(p_{1}, s_{1}\right)\right\rangle= & \bar{u}\left(p_{2}, s_{2}\right)\left[\gamma^{\mu} F_{1}(t)-i \sigma^{\mu \nu} \frac{r_{\nu}}{2 M} F_{2}(t)\right] \\
& \times u\left(p_{1}, s_{1}\right),
\end{aligned}
$$

where $\bar{u}\left(p_{2}, s_{2}\right)$ and $u\left(p_{1}, s_{1}\right)$ are the Dirac spinors, $r=p_{1}-p_{2}$ is the overall momentum transfer and the invariant $t=r^{2}$. The elastic form factors should not be confused with the dimensionless structure functions $F_{1}\left(x_{B}, Q^{2}\right)$ and $F_{2}\left(x_{B}, Q^{2}\right)$ introduced in the preceding chapter. Note also that for elastic scattering, we have $Q^{2}=-t$. The nucleon mass $M$ in Eq. (50) is introduced only for dimensional convenience. Similarly to the flavor decomposition of the electromagnetic current given by Eq. (49), the Dirac and Pauli form factors can also be expressed in terms of their flavor components,

$$
\begin{aligned}
& F_{1}(t)=\sum_{f} Q_{f} F_{1 f}(t), \\
& F_{2}(t)=\sum_{f} Q_{f} F_{2 f}(t) .
\end{aligned}
$$

Their limiting values at $t=0$ are known. The Dirac form factor gives the total electric charge of the nucleon $N, F_{1}(t=0)=Q_{N}$, and the Pauli form factor gives its anomalous magnetic moment, $F_{1}(t=0)=\kappa_{N}$. For the proton, we have $\kappa_{p}=1.793$, and for the neutron $\kappa_{n}=-1.913$. The $Q^{2}$-dependence of form factors is a clear evidence for the extended structure of the nucleon, in particular the charge and current distributions.

Note that writing the matrix element $\left\langle N\left(p_{2}, s_{2}\right)\left|J_{E M}^{\mu}(0)\right| N\left(p_{1}, s_{1}\right)\right\rangle$ in the most general form, one might expect to include, in addition to terms $\gamma^{\mu}$ and $\sigma^{\mu \nu} r_{\nu}$, also terms like $\left(p_{1}+p_{2}\right)^{\mu}, r^{\mu}$ and $\sigma^{\mu \nu}\left(p_{1}+p_{2}\right)_{\nu}$. However, with the help of the Gordon identity,

$$
\bar{u}\left(p_{2}, s_{2}\right) \gamma^{\mu} u\left(p_{1}, s_{1}\right)=\frac{1}{2 M} \bar{u}\left(p_{2}, s_{2}\right)\left[\left(p_{1}+p_{2}\right)^{\mu}-i \sigma^{\mu \nu} r_{\nu}\right] u\left(p_{1}, s_{1}\right),
$$

one can express the terms $\left(p_{1}+p_{2}\right)^{\mu}$ as the linear combination of terms $\gamma^{\mu}$ and $\sigma^{\mu \nu} r_{\nu}$. Moreover, the electromagnetic current conservation, $r_{\mu} J_{E M}^{\mu}=0$, implies that the term $r^{\mu}$ should vanish and hence the most general form reduces to the expression $(50)$. 
Instead of $F_{1}$ and $F_{2}$, we often introduce the so-called Sachs electric and magnetic form factors, $G_{E}$ and $G_{M}$, respectively. Then the matrix element takes the form

$$
\begin{aligned}
\left\langle N\left(p_{2}, s_{2}\right)\left|J_{E M}^{\mu}(0)\right| N\left(p_{1}, s_{1}\right)\right\rangle= & \bar{u}\left(p_{2}, s_{2}\right)\left[\gamma^{\mu} \frac{G_{E}(t)-\left(t / 4 M^{2}\right) G_{M}(t)}{1-t / 4 M^{2}}\right. \\
& \left.-i \sigma^{\mu \nu} \frac{r_{\nu}}{2 M} \frac{G_{M}(t)-G_{E}(t)}{1-t / 4 M^{2}}\right] u\left(p_{1}, s_{1}\right),
\end{aligned}
$$

where

$$
G_{E}(t)=F_{1}(t)+\frac{t}{4 M^{2}} F_{2}(t)
$$

and

$$
G_{M}(t)=F_{1}(t)+F_{2}(t)
$$

These form factors are normalized at $Q^{2}=0$ in the following way

$$
\begin{aligned}
G_{E p}(t=0) & =1 \\
G_{M p}(t=0) & =\mu_{p}=2.793
\end{aligned}
$$

for the proton and

$$
\begin{aligned}
G_{E n}(t=0) & =0 \\
G_{M n}(t=0) & =\mu_{n}=-1.913
\end{aligned}
$$

for the neutron. Their magnetic moments are given in terms of the nuclear magneton, $\mu=|e| / 2 M_{p}=5.051 \cdot 10^{-27} \mathrm{Am}^{2}$ in the SI units.

The nucleon electromagnetic form factors can be measured through elastic electron-nucleon scattering,

$$
e^{-}(k)+N\left(p_{1}\right) \longrightarrow e^{-}\left(k^{\prime}\right)+N\left(p_{2}\right)
$$

The process is shown in the one-photon exchange approximation in Fig. 4.

\section{III.3 PARTON DISTRIBUTION FUNCTIONS}

Parton distribution functions (PDFs) are defined through the forward matrix elements of the light-like correlation functions, i.e. the quark and gluon fields separated by 


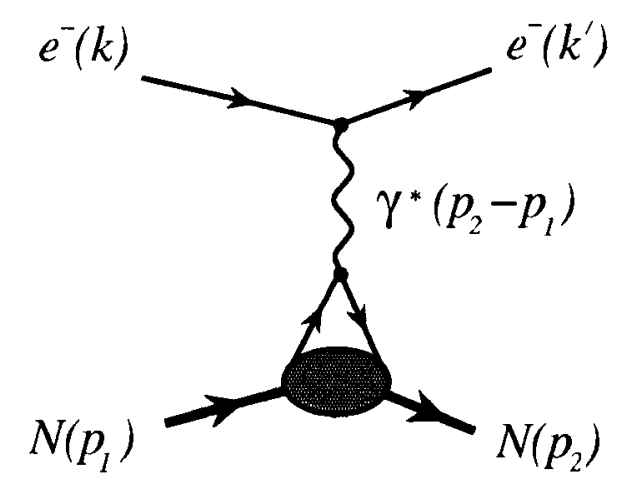

FIG. 4: Elastic electron-nucleon scattering in the one-photon exchange approximation.

light-like distances. For the unpolarized case we have

$$
\begin{array}{r}
\left\langle N(P, S)\left|\bar{\psi}_{f}(-z / 2) \gamma^{\mu} \psi_{f}(z / 2)\right| N(P, S)\right\rangle_{z^{2}=0}=\bar{u}(P, S) \gamma^{\mu} u(P, S) \\
\times \int_{0}^{1} d x\left[e^{i x(P \cdot z)} f_{N}(x)-e^{-i x(P \cdot z)} \bar{f}_{N}(x)\right],
\end{array}
$$

and for the polarized one

$$
\begin{array}{r}
\left\langle N(P, S)\left|\bar{\psi}_{f}(-z / 2) \gamma^{\mu} \gamma_{5} \psi_{f}(z / 2)\right| N(P, S)\right\rangle_{z^{2}=0}=\bar{u}(P, S) \gamma^{\mu} \gamma_{5} u(P, S) \\
\times \int_{0}^{1} d x\left[e^{i x(P \cdot z)} \Delta f_{N}(x)+e^{-i x(P \cdot z)} \Delta \bar{f}_{N}(x)\right]
\end{array}
$$

To make connection with GPDs, which are usually discussed in the region $-1 \leq x \leq 1$ (the variable $x$ runs from 0 to 1 for quarks and from -1 to 0 for antiquarks), it is convenient to introduce new distribution functions,

$$
\tilde{f}_{N}(x)=\left\{\begin{array}{cc}
f_{N}(x) & x>0 \\
-\bar{f}_{N}(-x) & x<0
\end{array}\right.
$$

and

$$
\Delta \tilde{f}_{N}(x)=\left\{\begin{array}{cc}
\Delta f_{N}(x) & x>0 \\
\Delta \bar{f}_{N}(-x) & x<0
\end{array}\right.
$$

and alternatively, write the integrals over $x$ in Eqs. (59) and (60) as

$$
\begin{aligned}
\int_{0}^{1} d x\left[e^{i x(P \cdot z)} f_{N}(x)-e^{-i x(P \cdot z)} \bar{f}_{N}(x)\right] & =\int_{-1}^{1} d x e^{i x(P \cdot z)} \tilde{f}_{N}(x) \\
\int_{0}^{1} d x\left[e^{i x(P \cdot z)} \Delta f_{N}(x)+e^{-i x(P \cdot z)} \Delta \bar{f}_{N}(x)\right] & =\int_{-1}^{1} d x e^{i x(P \cdot z)} \Delta \tilde{f}_{N}(x) .
\end{aligned}
$$


Furthermore, we observe that the definition of PDFs has the form of the planewave decomposition. Thus it allows to give the momentum-space interpretation. For example, $f_{N}(x)\left(\bar{f}_{N}(x)\right)$ is the probability to find the quark (antiquark) of flavor $f$ carrying the momentum $x P$ inside a fast-moving nucleon $N$ having the momentum $P$.

Parton distribution functions have been intensively studied in hard inclusive processes for the last three decades. The classic example in this respect is the deeply inelastic scattering process shown in Fig. 1. Its structure functions are directly expressed in terms of PDFs. By substituting Eq. (18) into the expression (6), it is easy to see that the DIS cross section is given, via the optical theorem, by the imaginary part of the forward virtual Compton scattering amplitude.

Let us consider this amplitude in the specific kinematics known as the Bjorken limit. Here the invariant momentum transfer to the nucleon system is sufficiently large, $-q^{2} \equiv Q^{2} \rightarrow \infty$, together with large total center-of-mass energy of the virtual photon-nucleon system, $s \equiv(P+q)^{2} \rightarrow \infty$, while the Bjorken ratio $x_{B} \equiv Q^{2} / 2(P \cdot q)$ is finite. In the deeply inelastic region discussed in Section II.3, we have, for instance, $Q^{2} \geq 1 \mathrm{GeV}^{2}$ and $W^{2} \equiv s \geq 4 \mathrm{GeV}^{2}$. Hence in this particular regime, the behavior of the forward VCA is dominated by short distances, i.e. when the separation between the two point-like photon-quark vertices (note that the photons couple to the quarks of the nucleon) in the amplitude is light-like. As a result, QCD factorization works and the amplitude factorizes into a convolution of a perturbatively calculable hard scattering process at the level of quarks and gluons, and process independent matrix elements, containing the soft nonperturbative information about the nucleon structure. These matrix elements are parametrized in terms of PDFs. Schematically, factorization allows to write the leading-order amplitude in the form of the so-called handbag diagrams. In these diagrams, two photons couple to the same quark line, as illustrated in Fig. 5. It is worth noting at this point that throughout this thesis we work only to the leading order in the strong coupling $\alpha_{s}$. Perturbative corrections produce logarithmic dependence of PDFs on the scale $Q^{2}$, in other words, they define the evolution of parton distributions, which can be calculated in QCD [57, 58, 59].

Now taking the imaginary part of the forward VCA generates the delta function,

$$
\Im \frac{1}{(x P \pm q)^{2}+i \epsilon}=-\frac{\pi}{2(P \cdot q)} \delta\left(x \mp x_{B}\right)
$$

which selects two points, $x= \pm x_{B}$, after the integration over the momentum fraction $x$. Thus in the DIS process we measure parton distribution functions $\tilde{f}_{N}(x)$ and 

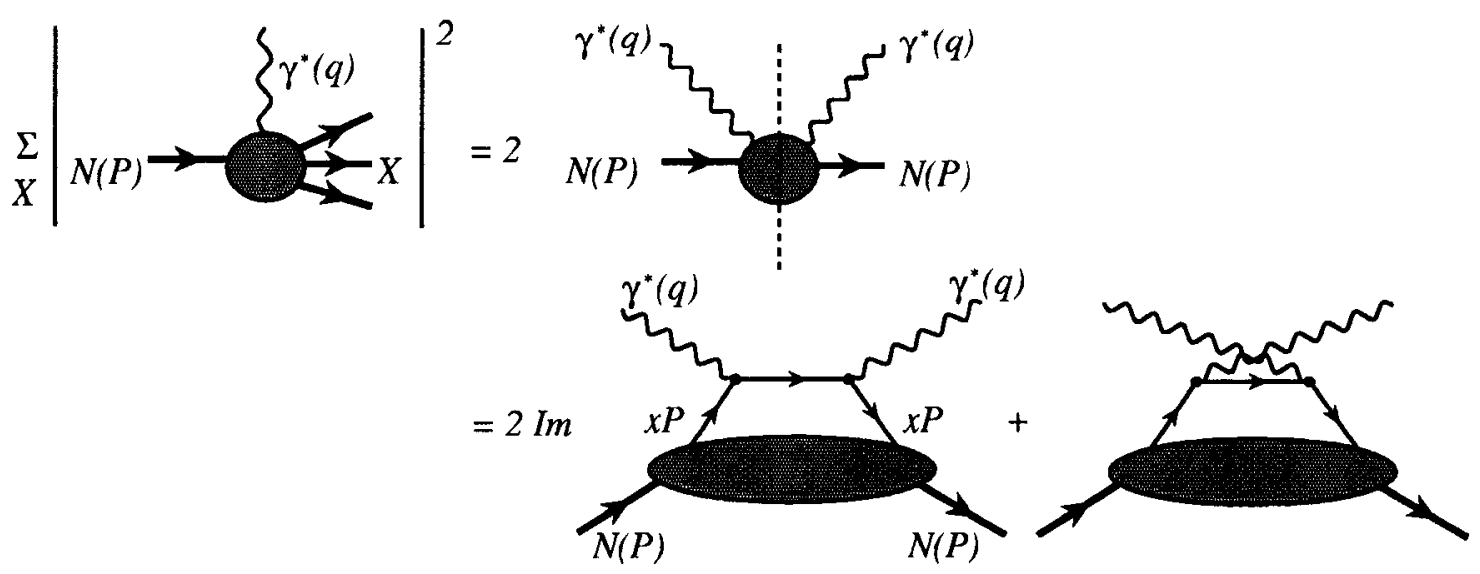

FIG. 5: Deeply inelastic electron-nucleon scattering cross section as the imaginary part of the forward virtual Compton scattering amplitude. The summation over $X$ reflects the inclusive nature of the nucleon structure description by parton distribution functions.

$\Delta \tilde{f}_{N}(x)$ at $x=x_{B}$ corresponding to the quark PDFs, and at $x=-x_{B}$ for those of antiquarks. Unlike the form factors, one deals, in the case of the parton distribution functions, with a light-like separation instead of a point vertex, and also the initial and final nucleon momenta are equal.

In summary, the unpolarized DIS experiments have mapped out the quark and gluon distributions in the nucleon while the polarized DIS experiments have shown that quarks carry a small fraction of the nucleon spin. As a result, new investigations to understand the nucleon spin became necessary.

\section{III.4 DISTRIBUTION AMPLITUDES}

Distribution amplitudes (DAs) (sometimes also referred to as the hadronic wave functions) $[60,61,62,63,64]$ describe hadrons in hard exclusive scattering processes and therefore, in addition to usual parton distribution functions, provide complementary information about the hadronic structure. They are defined through the vacuum-tohadron matrix elements $\langle 0|\ldots| P\rangle$ of light-cone operators. For example, in the pion case we write

$$
\left\langle 0\left|\bar{\psi}_{d}(-z / 2) \gamma^{\mu} \gamma_{5} \psi_{u}(z / 2)\right| \pi^{+}(P)\right\rangle_{z^{2}=0}=i P^{\mu} f_{\pi} \int_{-1}^{1} d \alpha e^{i \alpha(P \cdot z) / 2} \varphi_{\pi^{+}}(\alpha)
$$




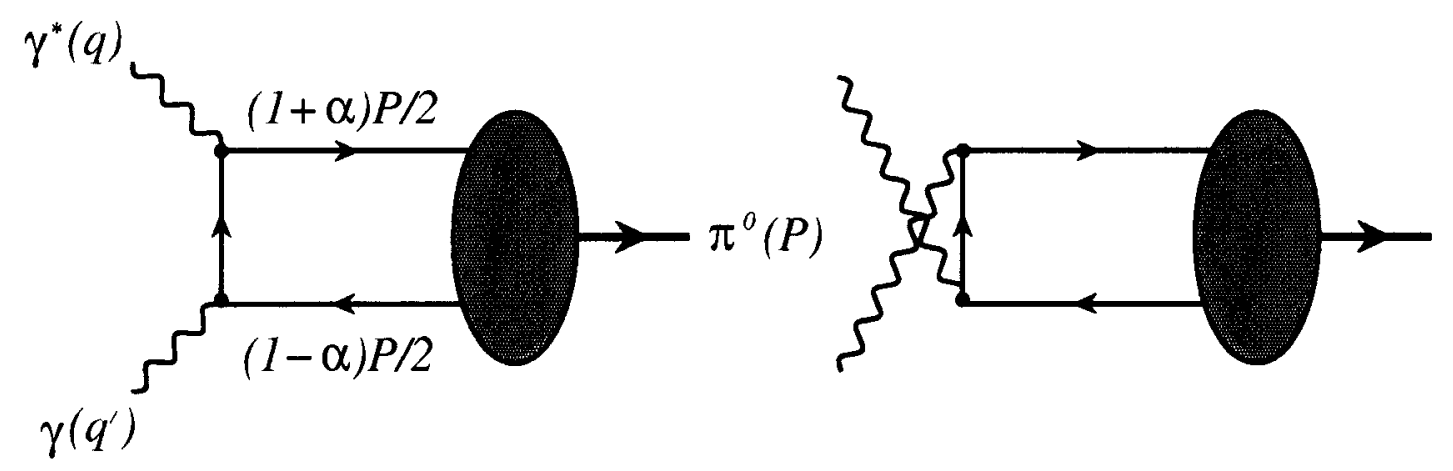

FIG. 6: The handbag contribution to the process $\gamma^{*} \gamma \rightarrow \pi^{0}$. The light-cone dominance is secured by the high virtuality of the incoming photon $\gamma^{*}$.

The fractions of the pion momentum carried by the quarks are $(1 \pm \alpha) / 2$. One can interpret $\varphi_{\pi^{+}}(\alpha)$ as the probability amplitude to find a positive fast-moving pion $\pi^{+}$ in a quark-antiquark state $\bar{u} d$, with the longitudinal pion momentum $P$ shared in fractions $(1+\alpha) / 2$ and $(1-\alpha) / 2$. The distribution $\varphi_{\pi^{+}}(\alpha)$ is an even function in the relative fraction $\alpha[54]$.

The simplest and cleanest process, in which the pion DA can be accessed, is the transition $[64,65]$

$$
\gamma^{*}(q)+\gamma\left(q^{\prime}\right) \longrightarrow \pi^{0}(P)
$$

For large virtuality $Q^{2}$, the leading-order contribution to the amplitude is given by the handbag diagrams depicted in Fig. 6.

\section{III.5 GENERALIZED PARTON DISTRIBUTIONS}

The key idea of the GPD approach is the so-called hybridization. As hybrids of form factors, PDFs and DAs, generalized parton distributions provide the most complete information about the hadronic structure. Parton distributions parametrize the longitudinal momentum distributions (in an infinite momentum frame) of partons in the hadron while the Fourier transforms of form factors in impact parameter space (impact parameter measures the transverse distance of the struck parton from the hadron center) describe the transverse coordinate distributions of the hadron's constituents $[66,67]$. Generalized parton distributions, on the other hand, encapsulate at the same time the longitudinal momentum and transverse coordinate distributions, 
thereby providing a much more detailed and comprehensive, three-dimensional snapshot of the substructure of the hadron. In addition, the universality of GPDs allows to develop a unified description of wide variety of different hard processes, both inclusive and exclusive.

Generalized parton distributions can be, in general, divided into two groups, namely, the skewed parton distributions (SPDs) and the double distributions (DDs).

\section{III.5.1 Skewed Parton Distributions}

There are two implementations of the SPD formalism, as illustrated in Fig. 7. The nonforward parton distributions (NFPDs) [34] have the advantage of using the variables similar to those of the usual PDFs. The distributions depend on $X$, the fraction of the plus component of the light-cone momentum $P^{+}$of the hadron carried by the parton (the plus and minus light-cone components are defined by $a^{ \pm} \equiv\left(a^{0} \pm a^{3}\right) / \sqrt{2}$ for any Lorentz four-vector $a$ ), or alternatively, the longitudinal momentum fraction with respect to the initial hadron momentum $P$; on the skewness parameter $\zeta$ specifying the difference between the initial and final hadron plus momenta, $r^{+}=\zeta P^{+}$; and on the invariant momentum transfer $t=r^{2}$. For instance, the nonforward parton distribution $F_{\zeta}^{f}(X, t)$ is the probability amplitude that the initial fast-moving hadron, having longitudinal momentum $P^{+}$, emits a parton of flavor $f$ carrying the momentum $X P^{+}$while the final hadron, having longitudinal momentum $(1-\zeta) P^{+}$, absorbs a parton of flavor $f$ carrying the momentum $(X-\zeta) P^{+}$. In this particular scheme, the initial and final hadron momenta are not treated symmetrically. The off-forward parton distributions (OFPDs) [30, 31], on the other hand, use symmetric variables expressed in terms of the average hadron momentum, $p=\left(p_{1}+p_{2}\right) / 2$, with $p_{1}$ being the momentum of the initial hadron and $p_{2}$ the momentum of the final one. In the symmetric scheme the hadron longitudinal (the plus component) momenta are $(1 \pm \xi) p^{+}$and accordingly, those of the active partons become $(x \pm \xi) p^{+}$. Similarly to NFPDs, the off-forward distributions are defined for each quark flavor, and are the functions of three variables, namely, the light-cone momentum fraction $x$, the skewness, $\xi \equiv r^{+} / 2 p^{+}$, (here the skewness is introduced as the coefficient of proportionality between the light-cone plus components of the momentum transfer and the average hadron momentum, and like $\zeta$ varies between 0 and 1) and the invariant $t$. In fact, one should bear in mind that both the nonforward and off-forward parton distributions also depend weakly (i.e. logarithmically) on the probing scale $Q^{2}$. 

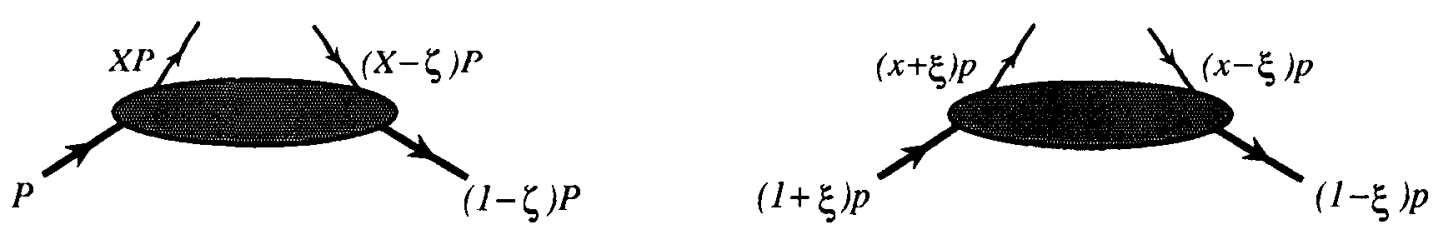

FIG. 7: Comparison between the descriptions in terms of nonforward and off-forward parton distribution functions.

The two pairs of variables $(X, \zeta)$ and $(x, \xi)$ are related to each other. The conversions between them are

$$
X=\frac{x+\xi}{1+\xi}
$$

and

$$
\zeta=\frac{2 \xi}{1+\xi}
$$

We emphasize that these variables solely characterize the plus, or the longitudinal, components of the momenta of the partons involved, however, their transverse momenta are related to the $t$-dependence of GPDs. The latter is driven by the $t$ dependence of the corresponding elastic form factors. Thus it is possible to access simultaneously the longitudinal momentum and transverse position of the parton in the infinite momentum frame [66, 67]. Moreover, by removing the parton with the light-cone momentum fraction $x+\xi$ from the hadron and replacing it, at some later point on the light-cone, with the parton of the momentum fraction $x-\xi$, one can say that SPDs (or GPDs, in general) measure the coherence between two different parton momentum states of the hadron whereas usual PDFs yield only the probability that a parton carries a fraction $x$ of the hadron momentum. In addition to the parton momentum as well as the spin correlations, we can even consider the matrix elements corresponding to different hadrons in the initial and final states, e.g. the proton-toneutron transition accessible through the exclusive charged pion electroproduction, the proton-to- $\Lambda$ transition in the kaon electroproduction, the nucleon-to-delta transition, and hence study flavor nondiagonal GPDs.

Let us focus on the off-forward parton distributions. At the leading, twist-2 level, the hadron structure information can be parametrized in terms of two unpolarized OFPDs, $H_{f}(x, \xi, t)$ and $E_{f}(x, \xi, t)$, and two polarized OFPDs, $\tilde{H}_{f}(x, \xi, t)$ 
and $\tilde{E}_{f}(x, \xi, t)$. Since $-1 \leq x \leq 1$ in Fig. 7 , the momentum fractions $x \pm \xi$ of the active partons can be either positive or negative. Positive and negative momentum fractions correspond to quarks and antiquarks, respectively. Therefore, each OFPD has three distinct regions. When $\xi \leq x \leq 1$, both partons represent quarks while for $-1 \leq x \leq-\xi$, they are both antiquarks. In these two regions, the distributions are just a generalization of the usual PDFs. In the central region, $-\xi \leq x \leq \xi$, which is often referred to as the mesonic region, the parton with a positive momentum $(x+\xi) p^{+}$ is going out from the blob and represents a quark. The returning parton has a negative momentum and therefore, should be treated as an outgoing antiquark with the momentum $(\xi-x) p^{+}$. The total momentum of the quark-antiquark pair, $r^{+}=2 \xi p^{+}$, is then shared in fractions $(x+\xi) p^{+}=(1+x / \xi) r^{+} / 2$ and $(\xi-x) p^{+}=(1-x / \xi) r^{+} / 2$. In this region of $x$, which is not present in deeply inelastic scattering, OFPDs behave like meson distribution amplitudes with $\alpha=x / \xi$.

Clearly, in the nonforward kinematics, SPDs uncover much richer information about hadronic structure, which is not accessible in the DIS process. This new information can be extracted with the study of hard exclusive processes, such as deeply exclusive photon or meson electroproduction (one refers to the former as deeply virtual Compton scattering or shortly DVCS), which turns out to be a much more difficult task due to the small cross sections. Nevertheless, high-energy and high-luminosity electron accelerators combined with large acceptance spectrometers give a unique opportunity to perform precision studies of such reactions.

The factorization into short and long distance dynamics is more general. Having large space-like virtuality of the initial photon is sufficient for QCD factorization to work $[31,34,68,69]$. In particular, in the DVCS process, which will be studied in detail in Chapter IV, the initial photon is highly virtual, $-q_{1}^{2} \rightarrow \infty$, while the final photon is on shell, $q_{2}^{2}=0$. In the leading-twist handbag approximation (DVCS is a handbag-dominated process for $-q_{1}^{2}$ as low as $2 \mathrm{GeV}^{2}$ ) illustrated in Fig. 8, the hard short-distance part of the so-called nonforward virtual Compton scattering amplitude factorizes from the nonperturbative long-distance part. The latter is represented by the lower blob and contains now the nonforward matrix elements $\left\langle N\left(p_{2}\right)|\ldots| N\left(p_{1}\right)\right\rangle$ of the same quark and gluon operators as in the forward case. These matrix elements, describing the nucleon structure, are parametrized in terms of GPDs. Even though the parton picture of DVCS, see Fig. 8, looks similar to that of DIS, see Fig. 5, there are three crucial differences. In DVCS, one deals with the skewed kinematics, 


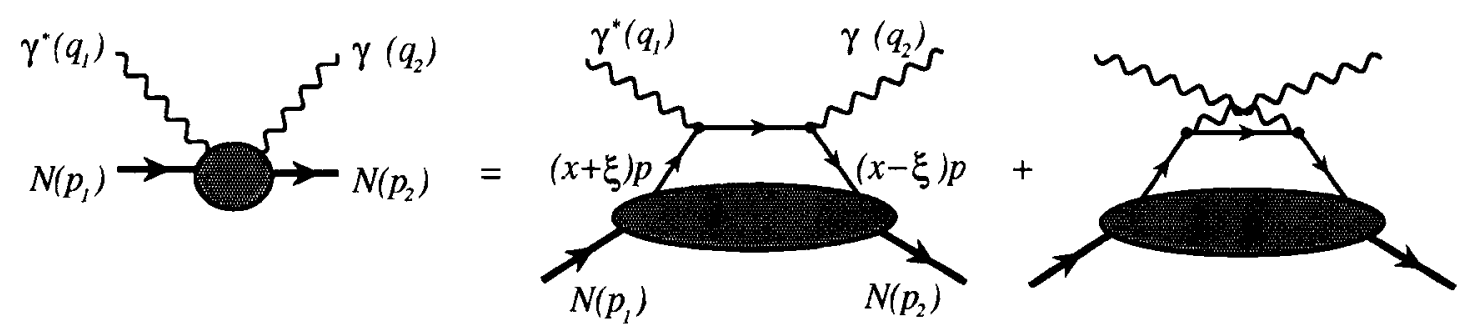

FIG. 8: Handbag contribution to the nonforward virtual Compton scattering amplitude. The hard quark propagator (this particular quark propagates along the light-cone) in the $s$ - and $u$-channel diagrams is convoluted with generalized parton distributions.

in which the plus momenta of the initial and final hadrons as well as partons are not equal. Furthermore, the invariant momentum transfer $t$ in DVCS is small but not zero. These two extra degrees of freedom, $\zeta$ (or $\xi$ ) and $t$, make the dynamics of DVCS rich and diverse. Finally, in DVCS (or in any other hard exclusive process) the virtual Compton scattering amplitude described by GPDs appears at the amplitude level whereas in DIS (or in any other inclusive process) the amplitude described by PDFs enters through the optical theorem at the level of the cross section.

Skewed parton distributions have interesting properties linking them to usual PDFs and form factors. In the forward limit, $p_{1}=p_{2}$ and $r=0, \xi=0, t=0$, they reduce to PDFs obtained from the DIS process. In particular, the OFPDs $H_{f}$ and $\tilde{H}_{f}$ coincide with the quark density distribution $f_{N}(x)$ and the quark helicity distribution $\Delta f_{N}(x)$ given by Eqs. (61) and (62). We write the so-called reduction formulas,

$$
H_{f}(x, 0,0)=\left\{\begin{array}{cc}
f_{N}(x) & x>0 \\
-\bar{f}_{N}(-x) & x<0
\end{array}\right.
$$

and

$$
\tilde{H}_{f}(x, 0,0)=\left\{\begin{array}{cc}
\Delta f_{N}(x) & x>0 \\
\Delta \bar{f}_{N}(-x) & x<0,
\end{array}\right.
$$

while the OFPDs $E_{f}$ and $\tilde{E}_{f}$ have no connections to PDFs. They are always accompanied with the momentum transfer $r$ and therefore, simply not visible in DIS. One can say that $E_{f}$ and $\tilde{E}_{f}$ encode completely new information on the hadron structure, which is not accessible in inclusive measurements. Even though they have no analogue in the forward limit like $H_{f}$ and $\tilde{H}_{f}$, their limits do exist. 
In the local limit, $z=0$, off-forward parton distributions reduce to the standard vector and axial vector form factors. In other words, the first moments of OFPDs, obtained by integrating the distributions over $x$, are equal to the nucleon elastic form factors (i.e. the Dirac, Pauli, axial and pseudoscalar form factors). For any $\xi$ one has the following relations for a particular quark flavor

$$
\begin{aligned}
\int_{-1}^{1} d x H_{f}(x, \xi, t) & =F_{1 f}(t), \\
\int_{-1}^{1} d x E_{f}(x, \xi, t) & =F_{2 f}(t), \\
\int_{-1}^{1} d x \tilde{H}_{f}(x, \xi, t) & =g_{A f}(t), \\
\int_{-1}^{1} d x \tilde{E}_{f}(x, \xi, t) & =g_{P f}(t) .
\end{aligned}
$$

We call these relations the sum rules. It is important to note that these sum rules are model and $\xi$-independent (the dependence on $\xi$ drops out after integration over $x)$.

Generalized parton distributions are also relevant for the nucleon spin structure and have received considerable attention in recent years in connection with the socalled proton spin puzzle (for reviews, see, e.g. [70, 71, 72]). Namely, certain low moments of GPDs can be related to the total angular momentum carried by quarks and gluons (or generically, partons) in the nucleon [30]. In particular, in terms of the off-forward distributions, the second moment of the unpolarized OFPDs at $t=0$ gives the quark total angular momentum,

$$
J_{q}=\frac{1}{2} \sum_{f} \int_{-1}^{1} d x x\left[H_{f}(x, \xi, t=0)+E_{f}(x, \xi, t=0)\right] .
$$

The above equation is independent of $\xi$ (again the $\xi$-dependence of $H_{f}$ and $E_{f}$ is removed by integration over $x$ ). The quark angular momentum, on the other hand, decomposes into the quark intrinsic spin $\Delta \Sigma$ and the quark orbital angular momentum $L_{q}$

$$
J_{q}=\frac{1}{2} \Delta \Sigma+L_{q}
$$

where $\Delta \Sigma$ is measured through the polarized DIS process. Substituting Eq. (72) into Eq. (73) we can determine $L_{q}$. Moreover, the total spin of the nucleon comes from quarks and gluons,

$$
\frac{1}{2}=J_{q}+J_{g}
$$


where $J_{g}$ is the total angular momentum carried by gluons. Thus we can also extract the gluon contribution to the nucleon spin. Note that $\Delta \Sigma / 2$ accounts only for approximately $30 \%$ of the nucleon spin. In summary, by measuring GPDs we obtain information about the angular momentum distributions of quarks and gluons in the nucleon. Therefore, the DVCS process, combined with measurements of the quark helicity distributions from inclusive deeply inelastic scattering, can unravel the orbital angular momentum carried by partons, on which little or no information is currently available.

\section{III.5.2 Double Distributions}

Two approaches are used to model generalized parton distributions:

- A direct calculation of GPDs in specific dynamical models, such as the bag model [73], the chiral soliton model [74], the light-cone formalism [39], etc.

- A phenomenological construction [75, 76, 77] based on reduction formulas relating GPDs to PDFs $f_{N}(x)$ and $\Delta f_{N}(x)$ and form factors $F_{1}(t), F_{2}(t), g_{A}(t)$ and $g_{P}(t)$.

The most convenient way to construct models in the second approach, and further study the interplay between $x, \xi$ and $t$ dependencies of GPDs is performed using the formalism of double distributions (DDs) $f(\beta, \alpha, t)$. Here we only consider the socalled $\alpha$-DDs corresponding to the symmetric description with respect to the initial and final hadron momenta. In the parton picture of double distributions shown in Fig. 9 , the active parton momentum, $k^{+}=\beta p^{+}+(1+\alpha) r^{+} / 2$, is represented as the sum of two components $\beta p^{+}$and $(1+\alpha) r^{+} / 2$. The former specifies the momentum flow in the $s$-channel due to the plus component of the average hadron momentum $p^{+}$, and the latter specifies the momentum flow in the $t$-channel due to the plus component of the momentum transfer $r^{+}$. Despite the proportionality between $r^{+}$and $p^{+}$, they correspond to the momentum fluxes in two different channels. Their superposition is the main feature of double distributions. In addition, it is important to note another characteristic feature, i.e. the absence of the $\xi$-dependence in $f(\beta, \alpha, t)$.

Thus double distributions are hybrids, which look like usual parton distributions with respect to the variable $\beta$, and like distribution amplitudes with respect to $\alpha$. Therefore, when modeling DDs we usually represent a double distribution in the factorized form as the product of a usual PDF in the $\beta$-direction and a distribution 


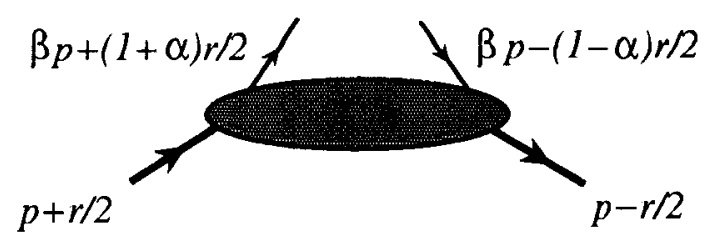

FIG. 9: Description in terms of double distributions.

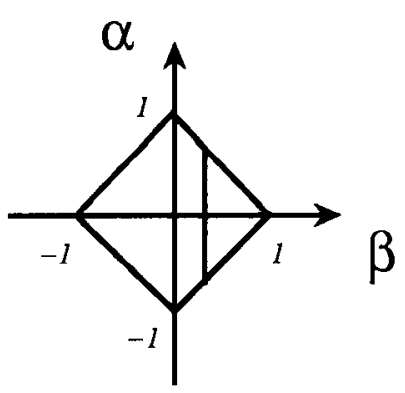

a

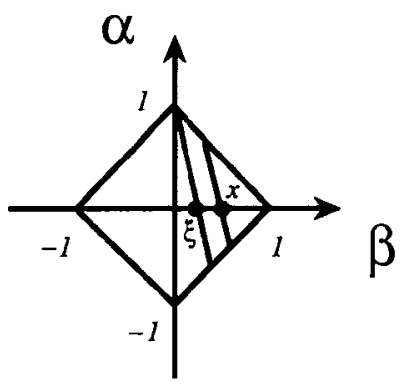

b

FIG. 10: Conversion from a double distribution to an off-forward parton distribution function with a zero and nonzero skewness.

amplitude, which drives the $\alpha$-profile. The connection between the DD variables $\alpha$ and $\beta$ and the OFPD variables $x$ and $\xi$ can be established through the formula $r^{+}=2 \xi p^{+}$, namely, $x=\beta+\xi \alpha$.

The support area of double distributions is the rhombus $|\alpha|+|\beta| \leq 1$. Moreover, due to the hermiticity properties and time-reversal invariance of the nonforward matrix elements, they are even functions in $\alpha, f(\beta, \alpha, t)=f(\beta,-\alpha, t)$. This property is termed as the Munich symmetry [76]. Now, in order to obtain the usual PDFs from DDs (recall that the former correspond to the forward limit, $\xi=0$ and $t=0$, of OFPDs), one should simply integrate them along the vertical lines $\beta=x$, see the rhombus (a) in Fig. 10. On the other hand, to get OFPDs $H(x, \xi, t)$ (we skip the flavor index for convenience) with a nonzero skewness (e.g. for $\xi=1$, they behave like meson distribution amplitudes), one should integrate DDs $f(\beta, \alpha, t)$ along the parallel lines $\alpha=(x-\beta) / \xi$ with a $\xi$-dependent slope,

$$
H(x, \xi, t)=\int_{-1}^{1} d \beta \int_{-1+|\beta|}^{1-|\beta|} d \alpha \delta(\beta+\xi \alpha-x) f(\beta, \alpha, t)
$$


as illustrated in the rhombus (b) in Fig. 10. We call this process of integration or scanning, the DD-tomography. To summarize, double distributions are primary objects producing SPDs after appropriate integration. 


\section{CHAPTER IV}

\section{DEEPLY VIRTUAL COMPTON SCATTERING}

\section{IV.1 INTRODUCTION}

In recent years, significant effort was made to access GPDs through the measurement of hard exclusive leptoproduction processes, such as deeply virtual Compton scattering or deeply exclusive meson production. The simplest process in this respect is DVCS. It can be accessed through the following reaction

$$
e^{-}(k)+N\left(p_{1}\right) \longrightarrow e^{-}\left(k^{\prime}\right)+N\left(p_{2}\right)+\gamma\left(q_{2}\right),
$$

and it is illustrated in Fig. 11. There are three relevant diagrams. The nucleon blob with two photon legs, see the diagram (a), represents the virtual Compton scattering amplitude, which will eventually become the subject of our study. This diagram is referred to as the DVCS diagram or the Compton contribution. Unfortunately, the final real photon can be emitted not only by the nucleon, but also by the electron. The latter is presented by the remaining two diagrams. They are referred to as the Bethe-Heitler contribution. Here lower part, the nucleon blob, stands for the electromagnetic form factor while the upper part can be exactly calculated in QED. Despite this disadvantage (in measuring of the VCA by extracting it from the cross section, the pure DVCS process is always in competition with the Bethe-Heitler process), in addition to a small cross section, deeply virtual Compton scattering is still regarded to be the cleanest tool to access the underlying GPDs.

At this point, we focus only on the Compton part. Hence an electron (or muon) scatters off a nucleon via the exchange (in the leading-order QED) of a space-like photon with virtuality $q_{1}^{2}=\left(k-k^{\prime}\right)^{2}<0$, producing an intact nucleon (with altered momentum) and a real photon in the final state. Since we turn a virtual photon into a real one there is always a nonzero momentum transfer. At the quark level, in leading twist, the electromagnetic current couples to different quark species with strength proportional to the squares of the quark charges, selecting specific linear combinations of GPDs. Flavor-specific GPDs can be reconstructed by considering DVCS from different hadrons (protons and neutrons, for instance), and using isospin or flavor symmetry to relate GPDs in the proton to those in the neutron. 


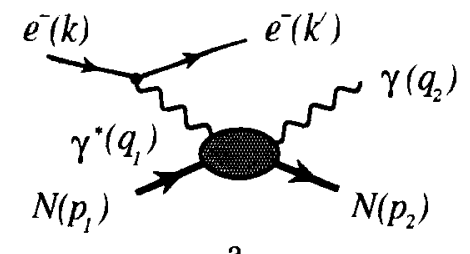

a

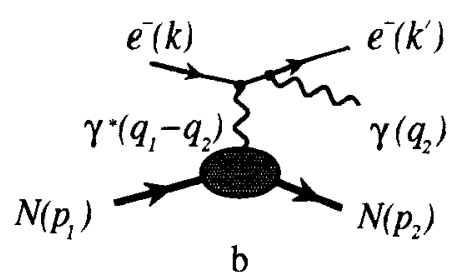

$\mathrm{b}$

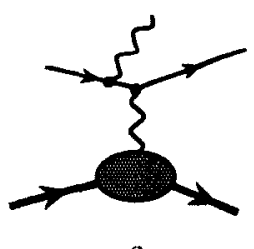

$\mathrm{c}$

FIG. 11: DVCS (a) and Bethe-Heitler (b and c) diagrams contributing to electroproduction of a real photon.

In the Bjorken regime (recall that in addition to the large invariant mass of the photon-nucleon system, $\left(p_{1}+q_{1}\right)^{2} \rightarrow \infty$, the initial photon is also highly virtual, nevertheless, their ratio $x_{B} \equiv-q_{1}^{2} / 2\left(p_{1} \cdot q_{1}\right)$ is finite), the VCA is dominated by light-like distances. The dominant light-cone singularities, which generate the leading power contributions in $1 /\left|q_{1}^{2}\right|$ to the amplitude, are represented by two handbag diagrams shown in Fig. 12, in which the (hard) quark propagator is convoluted with the soft function parametrized in terms of GPDs. In addition, keeping the momentum transfer squared to the nucleon, $t \equiv\left(p_{1}-p_{2}\right)^{2}$, as small as possible, one arrives at the DVCS kinematics, $s>-q_{1}^{2} \gg-t$. This particular kinematics implies, on one hand, that $-q_{1}^{2}$ should be large enough to ensure scaling regime for the amplitude and, on the other hand, it implies small $t$.

One of the methods to study the virtual Compton scattering amplitude in the DVCS kinematics is the approach based on the nonlocal light-cone expansion of the product of currents in QCD string operators in coordinate space [78]. It will be employed in the present work.

A detailed derivation of the leading-twist (and to the lowest order in $\alpha_{s}$ ) VCA is provided in Section IV.2. In Section IV.3, we discuss the kinematics, common to all DVCS-like reactions. After introducing the simple model for nucleon GPDs, see Section IV.4, both the Compton and Bethe-Heitler cross sections are estimated in Section IV.5.

\section{IV.2 VIRTUAL COMPTON SCATTERING AMPLITUDE}

We begin with an analysis of some general aspects of the VCA. In the most general nonforward case, the amplitude is given by a Fourier transform of the correlation 

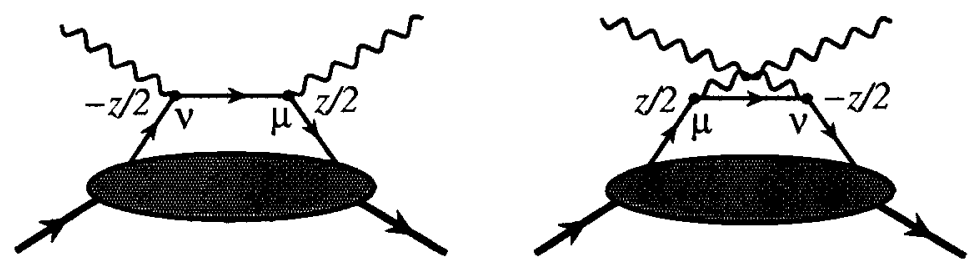

FIG. 12: Handbag diagrams ( $s$ - and $u$-channel) in the virtual Compton scattering amplitude.

function of two electroweak currents. In particular, for the standard virtual Compton process on the nucleon, where both currents are electromagnetic one has [54]

$$
T_{E M}^{\mu \nu}=i \int d^{4} x \int d^{4} y e^{-i\left(q_{1} \cdot x\right)+i\left(q_{2} \cdot y\right)}\left\langle N\left(p_{2}, s_{2}\right)\left|T\left\{J_{E M}^{\mu}(y) J_{E M}^{\nu}(x)\right\}\right| N\left(p_{1}, s_{1}\right)\right\rangle .
$$

Due to the current conservation, the amplitude is transverse with respect to the incoming virtual and outgoing real photon momenta,

$$
T_{E M}^{\mu \nu} q_{1 \nu}=0 \text { and } q_{2 \mu} T_{E M}^{\mu \nu}=0 .
$$

It will be convenient in the analysis to use symmetric coordinates, defined by introducing center and relative coordinates of the points $x$ and $y, X \equiv(x+y) / 2$ and $z \equiv y-x$. Accordingly, the amplitude takes the form

$$
\begin{aligned}
T_{E M}^{\mu \nu}= & i \int d^{4} X \int d^{4} z e^{-i\left(q_{1}-q_{2}\right) \cdot X+i\left(q_{1}+q_{2}\right) \cdot z / 2} \\
& \times\left\langle N\left(p_{2}, s_{2}\right)\left|T\left\{J_{E M}^{\mu}(X+z / 2) J_{E M}^{\nu}(X-z / 2)\right\}\right| N\left(p_{1}, s_{1}\right)\right\rangle .
\end{aligned}
$$

Furthermore, in order to treat the initial and final nucleons in a symmetric manner, we introduce, as independent momentum variables, the averages of the photon and nucleon momenta, $q \equiv\left(q_{1}+q_{2}\right) / 2$ and $p \equiv\left(p_{1}+p_{2}\right) / 2$, and the overall momentum transfer to the nucleon, $r \equiv p_{1}-p_{2}=q_{2}-q_{1}$. Then we can write

$$
q^{2}=q_{1}^{2} / 2-t / 4 \text { with } t=r^{2} .
$$

From the on-mass-shell condition, $p_{1}^{2}=p_{2}^{2}=M^{2}$, it follows that

$$
p^{2}=M^{2}-t / 4 \text { and }(p \cdot r)=0
$$

where $M$ denotes the mass of the nucleon. After performing the translation in $x$ in Eq. (79), $\left\langle p_{2}\left|J^{\mu}(x)\right| p_{1}\right\rangle=\left\langle p_{2}\left|J^{\mu}(0)\right| p_{1}\right\rangle e^{-i\left(p_{1}-p_{2}\right) \cdot x}$, and integrating over the center 
coordinate, one finds

$$
T_{E M}^{\mu \nu}=(2 \pi)^{4} \delta^{(4)}\left(p_{1}+q_{1}-p_{2}-q_{2}\right) \mathcal{T}_{E M}^{\mu \nu},
$$

where

$$
\mathcal{T}_{E M}^{\mu \nu}=i \int d^{4} z e^{i(q \cdot z)}\left\langle N\left(p-r / 2, s_{2}\right)\left|T\left\{J_{E M}^{\mu}(z / 2) J_{E M}^{\nu}(-z / 2)\right\}\right| N\left(p+r / 2, s_{1}\right)\right\rangle .
$$

We call the amplitude (83) the reduced virtual Compton scattering amplitude. It appears in the invariant matrix element (or alternatively the T-matrix) of the standard electromagnetic DVCS process.

Having introduced the reduced VCA we demonstrate its calculation at the twist-2 level in the DVCS kinematics, which amounts to neglecting contributions of the order $M^{2} / q^{2}$ and $t / q^{2}$ in the hard part of the amplitude, and keeping the $t$-dependence only in the soft part. It was noticed that a straightforward use of the twist-2 result for $t \neq 0$ leads to inconsistencies, e.g. the VCA is not electromagnetic gauge invariant [35]. The invariance is restored through inclusion of the twist-3 corrections to the amplitude. They are power suppressed in $q^{2}$, and have been calculated by several groups $[79,80,81,82,83,84,85,86,87,88,89]$ using different approaches.

Since the final state photon is on shell, $q_{2}^{2}=0$, it follows in this particular kinematics that $\left(r \cdot q_{1}\right) \simeq-q_{1}^{2} / 2=x_{B}\left(p_{1} \cdot q_{1}\right)$. Hence the momentum transfer should have a large component in the direction of the average nucleon momentum,

$$
r=2 \eta p+\Delta
$$

characterized by the skewness parameter,

$$
\eta \equiv \frac{(r \cdot q)}{2(p \cdot q)}
$$

In the DVCS kinematics, the remainder $\Delta$ in Eq. (84) is transverse to both $p$ and $q,(\Delta \cdot p)=-2 \eta p^{2} \rightarrow 0$ and $(\Delta \cdot q)=-t / 4 \rightarrow 0[82]$. In the language of the parton model, the component $2 \eta p$ of the momentum transfer would be identified with the longitudinal component $r_{\|}$while $\Delta$ would be a transverse component $r_{\perp}$ [83]. Moreover, $\eta$ coincides with another scaling variable $\xi$ defined as [30]

$$
\xi \equiv-\frac{q^{2}}{2(p \cdot q)}
$$


For that reason, $\xi$ in DVCS is frequently also referred to as skewness. It is easy to verify that $\xi=x_{B} /\left(2-x_{B}\right)$ in the limit $t / q^{2} \rightarrow 0$. In DIS, on the other hand, the asymptotic region, which is also characterized by the large virtuality of the incoming photon, corresponds to the situation in which $q_{1}=q_{2}, p_{1}=p_{2}, r=0$ and accordingly, $\eta=0$ and $\xi=x_{B}$.

Let us now turn to the formal light-cone expansion of the time-ordered product $T\left\{J_{E M}^{\mu}(z / 2) J_{E M}^{\nu}(-z / 2)\right\}$ in the coordinate representation. The expansion is performed in terms of QCD string operators, as discussed in Ref. [78]. The string operators have gauge links along the straight line between the fields (the gauge link connecting the two space-time points corresponds to the summation over twist- 0 longitudinal gluons, and it disappears, e.g. in the Fock-Schwinger gauge, $z^{\alpha} A_{\alpha}(z)=0$ ), however, for brevity we will not write them explicitly. The leading light-cone singularity, $z^{2} \rightarrow 0$, is given by the sum of two ( $s$ - and $u$-channel) handbag diagrams shown in Fig. 12. The hard part of each of the diagrams begins at zeroth order in $\alpha_{s}$ with the purely tree level diagrams, in which the virtual and real photons interact with the (massless) quarks. We have

$$
\begin{aligned}
i T\left\{J_{E M}^{\mu}(z / 2) J_{E M}^{\nu}(-z / 2)\right\}= & i \sum_{f} Q_{f}^{2}\left\{\bar{\psi}_{f}(z / 2) \gamma^{\mu} i \not \triangleright(z) \gamma^{\nu} \psi_{f}(-z / 2)\right. \\
& \left.+\bar{\psi}_{f}(-z / 2) \gamma^{\nu} i \not \supset(-z) \gamma^{\mu} \psi_{f}(z / 2)\right\}
\end{aligned}
$$

where $Q_{f}$ denotes the electric charge of the quark with flavor $f$ in units of $|e|$. Note that the vertices, in fact, contribute the factor $\left(-i|e| Q_{f}\right)^{2}$ rather than $Q_{f}^{2}$. For convenience, we do not include an extra factor of $-e^{2}$ in the expression for the VCA, however, it will be included in the T-matrix. The free quark propagator between the initial and final quark fields in the coordinate representation is given by

$$
\not(z)=\frac{\not}{2 \pi^{2}\left(z^{2}-i 0\right)^{2}}=\int \frac{d^{4} l}{(2 \pi)^{4}} e^{-i(l \cdot z)} \frac{l}{l^{2}+i 0} .
$$

Using the $\gamma$-matrix formula,

$$
\gamma^{\mu} \gamma^{\rho} \gamma^{\nu}=\left(s^{\mu \rho \nu \eta}+i \epsilon^{\mu \rho \nu \eta} \gamma_{5}\right) \gamma_{\eta}
$$

where $s^{\mu \rho \nu \eta} \equiv g^{\mu \rho} g^{\nu \eta}+g^{\mu \eta} g^{\rho \nu}-g^{\mu \nu} g^{\rho \eta}$ is the symmetric and $\epsilon^{\mu \rho \nu \eta}$ is the antisymmetric tensor in the Lorentz indices $\mu$ and $\nu$, we express the original bilocal quark operators in Eq. (87) in terms of operators with only one Lorentz index,

$$
i T\left\{J_{E M}^{\mu}(z / 2) J_{E M}^{\nu}(-z / 2)\right\}=
$$




$$
\begin{aligned}
\frac{z_{\rho}}{2 \pi^{2} z^{4}} \sum_{f} Q_{f}^{2}\left\{s^{\mu \rho \nu \eta}\left[\bar{\psi}_{f}(-z / 2) \gamma_{\eta} \psi_{f}(z / 2)-\bar{\psi}_{f}(z / 2) \gamma_{\eta} \psi_{f}(-z / 2)\right]\right. \\
\left.+i \epsilon^{\mu \rho \nu \eta}\left[\bar{\psi}_{f}(-z / 2) \gamma_{\eta} \gamma_{5} \psi_{f}(z / 2)+\bar{\psi}_{f}(z / 2) \gamma_{\eta} \gamma_{5} \psi_{f}(-z / 2)\right]\right\}
\end{aligned}
$$

It is customary to write these new QCD bilocal operators (the vector and axial vector string operators) as

$$
\begin{aligned}
\mathcal{O}_{\eta}^{f}(z \mid 0) & =\left[\bar{\psi}_{f}(-z / 2) \gamma_{\eta} \psi_{f}(z / 2)-\bar{\psi}_{f}(z / 2) \gamma_{\eta} \psi_{f}(-z / 2)\right] \\
\mathcal{O}_{5 \eta}^{f}(z \mid 0) & =\left[\bar{\psi}_{f}(-z / 2) \gamma_{\eta} \gamma_{5} \psi_{f}(z / 2)+\bar{\psi}_{f}(z / 2) \gamma_{\eta} \gamma_{5} \psi_{f}(-z / 2)\right]
\end{aligned}
$$

and accordingly, Eq. (90) turns into

$$
i T\left\{J_{E M}^{\mu}(z / 2) J_{E M}^{\nu}(-z / 2)\right\}=\frac{z_{\rho}}{2 \pi^{2} z^{4}} \sum_{f} Q_{f}^{2}\left\{s^{\mu \rho \nu \eta} \mathcal{O}_{\eta}^{f}(z \mid 0)+i \epsilon^{\mu \rho \nu \eta} \mathcal{O}_{5 \eta}^{f}(z \mid 0)\right\} .
$$

The string operators in Eq. (91) do not have a definite twist. To isolate their twist-2 part one uses a Taylor series expansion of $\mathcal{O}_{\eta}^{f}(z \mid 0)$ and $\mathcal{O}_{5 \eta}^{f}(z \mid 0)$ in the relative coordinate $z$. This gives local operators, $\bar{\psi}_{f}(0) \gamma_{\eta} D_{\mu_{1}} \ldots D_{\mu_{n}} \psi_{f}(0)$ and $\bar{\psi}_{f}(0) \gamma_{\eta} \gamma_{5} D_{\mu_{1}} \ldots D_{\mu_{n}} \psi_{f}(0)$, where $D_{\mu}$ is the covariant derivative, which are not symmetric in their indices. To get the twist- 2 contribution, one should keep only the totally symmetric traceless parts of the coefficients in the expansion. As it was shown in Ref. [78], the totally symmetric parts can be carried out by the following operation

$$
\begin{aligned}
{\left[\mathcal{O}_{\eta}^{f}(z \mid 0)\right]_{s y m}=} & \partial_{\eta} \int_{0}^{1} d \beta \\
& \times\left[\bar{\psi}_{f}(-\beta z / 2) \not \psi_{f}(\beta z / 2)-\bar{\psi}_{f}(\beta z / 2) \not z \psi_{f}(-\beta z / 2)\right], \\
{\left[\mathcal{O}_{5 \eta}^{f}(z \mid 0)\right]_{s y m}=} & \partial_{\eta} \int_{0}^{1} d \beta \\
& \times\left[\bar{\psi}_{f}(-\beta z / 2) \not z \gamma_{5} \psi_{f}(\beta z / 2)+\bar{\psi}_{f}(\beta z / 2) \not z \gamma_{5} \psi_{f}(-\beta z / 2)\right],
\end{aligned}
$$

where $\partial_{\eta} \equiv \partial / \partial z^{\eta}$ is the derivative with respect to the relative coordinate. It becomes clear now why the term string is used: the argument of $\psi_{f}$ and $\bar{\psi}_{f}$ takes all the values on the string from $-z / 2$ to $z / 2$. The subtraction of traces can be achieved by imposing the harmonic condition on the contracted vector and axial vector string operators,

$$
\begin{aligned}
& \mathcal{O}^{f+}(z) \equiv z^{\eta} \mathcal{O}_{\eta}^{f}(z \mid 0)=\left[\bar{\psi}_{f}(-z / 2) \not z \psi_{f}(z / 2)+(z \rightarrow-z)\right], \\
& \mathcal{O}_{5}^{f-}(z) \equiv z^{\eta} \mathcal{O}_{5 \eta}^{f}(z \mid 0)=\left[\bar{\psi}_{f}(-z / 2) \not z \gamma_{5} \psi_{f}(z / 2)-(z \rightarrow-z)\right],
\end{aligned}
$$


which appear on the right-hand-side of Eq. (93). In other words, these two operators should satisfy d'Alembert equation with respect to $z$

$$
\partial^{2}\left[\mathcal{O}^{f+}(z)\right]_{t w i s t-2}=0
$$

and similarly for the twist-2 part of $\mathcal{O}_{5}^{f-}(z)$. Note that we have assigned an extra superscript \pm to the operators (94) because they possess the symmetry with respect to the change $z \rightarrow-z$.

To compute the amplitude (83), the contracted twist-2 operators have to be sandwiched between the initial and final nucleon states, and ultimately integrated over $z$. Thus we need to construct a parametrization for these nonforward nucleon matrix elements. The most convenient way is a decomposition into plane waves, i.e. a spectral representation, where the relevant spectral functions correspond to GPDs (to be specific, here and in the following, we use the off-forward parton distributions). In principle, we need to provide a parametrization valid everywhere in $z$ since the coordinate $z$ runs over the whole four-dimensional space. However, it turns out that the inclusion of the $z^{2}$ terms in the matrix elements generate $M^{2} / q^{2}$ and $t / q^{2}$ corrections to the amplitude (such corrections are analogous to the well-known target mass corrections in DIS $[90,91])$ and hence will be neglected. It is, therefore, sufficient to provide a parametrization only on the light-cone [83], namely,

$$
\begin{aligned}
\left\langle N\left(p_{2}, s_{2}\right)\left|\mathcal{O}^{f+}(z)\right| N\left(p_{1}, s_{1}\right)\right\rangle_{z^{2}=0}= & \bar{u}\left(p_{2}, s_{2}\right) \not u\left(p_{1}, s_{1}\right) \\
& \times \int_{-1}^{1} d x e^{i x(p \cdot z)} H_{f}^{+}(x, \xi, t) \\
& +\bar{u}\left(p_{2}, s_{2}\right) \frac{(\not f \gamma-\not f z)}{4 M} u\left(p_{1}, s_{1}\right) \\
& \times \int_{-1}^{1} d x e^{i x(p \cdot z)} E_{f}^{+}(x, \xi, t), \\
\left\langle N\left(p_{2}, s_{2}\right)\left|\mathcal{O}_{5}^{f-}(z)\right| N\left(p_{1}, s_{1}\right)\right\rangle_{z^{2}=0}= & \bar{u}\left(p_{2}, s_{2}\right) \not z \gamma_{5} u\left(p_{1}, s_{1}\right) \\
& \times \int_{-1}^{1} d x e^{i x(p \cdot z)} \tilde{H}_{f}^{+}(x, \xi, t) \\
& -\bar{u}\left(p_{2}, s_{2}\right) \frac{(r \cdot z)}{2 M} \gamma_{5} u\left(p_{1}, s_{1}\right) \\
& \times \int_{-1}^{1} d x e^{i x(p \cdot z)} \tilde{E}_{f}^{+}(x, \xi, t) .
\end{aligned}
$$

The flavor dependent OFPDs in the parametrization (96) refer to the corresponding quark flavor $f$ in the nucleon $N$. Apart from the scale $Q^{2} \equiv-q^{2}$, there are three variables necessary to specify OFPDs, namely, the usual light-cone momentum fraction $x$, the invariant momentum transfer $t$ to the target and the skewness parameter 
$\xi$, which corresponds to the light-cone momentum fraction transferred to the target and characterizes the momentum asymmetry. Recall that the variables $x$ and $\xi$ only define the longitudinal momenta of the partons involved, that is their plus components. Schematically, the parton going out of the parent nucleon in Fig. 12 carries the fraction $(x+\xi)$ of the average nucleon momentum $p$ while the momentum of the returning parton is $(x-\xi) p$.

The OFPDs $H_{f}(x, \xi, t), E_{f}(x, \xi, t), \tilde{H}_{f}(x, \xi, t)$ and $\tilde{E}_{f}(x, \xi, t)$, introduced in Section III.5, parametrize the matrix elements of operators $\bar{\psi}_{f}(-z / 2) \not k \psi_{f}(z / 2)$ and $\bar{\psi}_{f}(-z / 2) \not k \gamma_{5} \psi_{f}(z / 2)$, do not have symmetry with respect to the change $x \rightarrow-x$. In particular, the function $H_{f}(x)$ in the forward limit, see Eq. (69), corresponds for positive $x$ to the quark distribution while for negative $x$, it corresponds to the minus antiquark distribution. On the other hand, the plus distributions, introduced through the matrix elements of (anti)symmetrized operators given by Eq. (94), do have well defined symmetry properties with respect the scaling variable $x$. Simply by transforming $z \rightarrow-z$ and $x \rightarrow-x$ in Eq. (96), we can establish the following

$$
\begin{aligned}
H_{f}^{+}(x) & =-H_{f}^{+}(-x), \\
E_{f}^{+}(x) & =-E_{f}^{+}(-x), \\
\tilde{H}_{f}^{+}(x) & =\tilde{H}_{f}^{+}(-x), \\
\tilde{E}_{f}^{+}(x) & =\tilde{E}_{f}^{+}(-x) .
\end{aligned}
$$

It is easy to notice that the plus OFPDs are determined by the sum of quark and antiquark distributions. Since the quark distribution includes both the valence and sea contributions, and further the antiquark distribution only the sea contribution, the plus distribution turns into the sum of the valence and twice the sea quark distributions.

After substitution of Eq. (96) into the right-hand side of Eq. (93), we first take the derivative with respect to $z$. Then we perform integration by parts over the parameter $\beta$ and keep only the surface terms with the arguments $\bar{\psi}_{f}( \pm z / 2)$ and $\psi_{f}( \pm z / 2)$. Finally, the integral over $z$ is carried out with the help of the inversion formula for $\$(z)$,

$$
\int d^{4} z e^{i(l \cdot z)} \frac{z_{\rho}}{2 \pi^{2}\left(z^{2}-i 0\right)^{2}}=\frac{l_{\rho}}{\left(l^{2}+i 0\right)} .
$$

Here the momentum $l$ is given by $l=(x p+q)$, so that $l^{2}$ in the denominator of Eq. 
(98) becomes $l^{2}=2(p \cdot q)(x-\xi)$. The expression for the reduced VCA reads

$$
\begin{array}{r}
\mathcal{T}_{\text {EMtwist }-2}^{\mu \nu}=\frac{1}{2(p \cdot q)} \sum_{f} Q_{f}^{2} \int_{-1}^{1} \frac{d x}{(x-\xi+i 0)} \\
\times\left\{H _ { f } ^ { + } ( x , \xi , t ) \left[l^{\mu} \bar{u}\left(p_{2}, s_{2}\right) \gamma^{\nu} u\left(p_{1}, s_{1}\right)\right.\right. \\
\left.+l^{\nu} \bar{u}\left(p_{2}, s_{2}\right) \gamma^{\mu} u\left(p_{1}, s_{1}\right)-g^{\mu \nu} \bar{u}\left(p_{2}, s_{2}\right) \not u\left(p_{1}, s_{1}\right)\right] \\
+E_{f}^{+}(x, \xi, t)\left[l^{\mu} \bar{u}\left(p_{2}, s_{2}\right) \frac{\gamma^{\nu} \gamma-\not \gamma^{\nu}}{4 M} u\left(p_{1}, s_{1}\right)\right. \\
\left.+l^{\nu} \bar{u}\left(p_{2}, s_{2}\right) \frac{\gamma^{\mu} \gamma-\not \gamma^{\mu}}{4 M} u\left(p_{1}, s_{1}\right)-g^{\mu \nu} \bar{u}\left(p_{2}, s_{2}\right) \frac{(\not \gamma-\gamma \not l)}{4 M} u\left(p_{1}, s_{1}\right)\right] \\
-i \epsilon^{\mu \nu \rho \eta}\left[\tilde{H}_{f}^{+}(x, \xi, t) l_{\rho} \bar{u}\left(p_{2}, s_{2}\right) \gamma_{\eta} \gamma_{5} u\left(p_{1}, s_{1}\right)\right. \\
\left.\left.-\tilde{E}_{f}^{+}(x, \xi, t) l_{\rho} \bar{u}\left(p_{2}, s_{2}\right) \frac{r_{\eta}}{2 M} \gamma_{5} u\left(p_{1}, s_{1}\right)\right]\right\}
\end{array}
$$

and can be further simplified as follows. Let us perform the light-cone decomposition of $\gamma$-matrix,

$$
\gamma^{\mu}=a^{\mu} \not n_{1}+b^{\mu} \not n_{2}+\gamma_{\perp}^{\mu}
$$

The four-vectors $n_{1}$ and $n_{2}$ in Eq. (100) are light-like, $n_{1}^{2}=n_{2}^{2}=0$, and satisfy the condition $\left(n_{1} \cdot n_{2}\right)=1$. Identifying $n_{1} \rightarrow p, n_{2} \rightarrow q_{2}$ and neglecting the transverse component $\gamma_{\perp}^{\mu}$, since it corresponds to the higher-twist contributions, Eq. (100) takes the form

$$
\gamma^{\mu}=\frac{1}{\left(p \cdot q_{2}\right)}\left(q_{2}^{\mu} \not p+p^{\mu} \phi_{2}\right)
$$

Using the above decomposition, the Dirac equation, $p_{1} u\left(p_{1}, s_{1}\right)=p_{2} u\left(p_{2}, s_{2}\right)=0$ (recall that we neglect the nucleon mass), and the fact that $H_{f}^{+}$and $E_{f}^{+}$are odd functions in $x$, the reduced VCA in the leading twist transforms into

$$
\begin{array}{r}
\mathcal{T}_{E M t w i s t-2}^{\mu \nu}=\frac{1}{2(p \cdot q)} \sum_{f} Q_{f}^{2} \int_{-1}^{1} \frac{d x}{(x-\xi+i 0)} \\
\times\left\{H_{f}^{+}(x, \xi, t)\left[\frac{1}{\left(p \cdot q_{2}\right)}\left(p^{\mu} q_{2}^{\nu}+p^{\nu} q_{2}^{\mu}\right)-g^{\mu \nu}\right] \bar{u}\left(p_{2}, s_{2}\right) q_{2} u\left(p_{1}, s_{1}\right)\right. \\
+E_{f}^{+}(x, \xi, t)\left[\frac{1}{\left(p \cdot q_{2}\right)}\left(p^{\mu} q_{2}^{\nu}+p^{\nu} q_{2}^{\mu}\right)-g^{\mu \nu}\right] \bar{u}\left(p_{2}, s_{2}\right) \frac{\left(d_{2} \gamma-\not q_{2}\right)}{4 M} u\left(p_{1}, s_{1}\right) \\
-\tilde{H}_{f}^{+}(x, \xi, t)\left[\frac{1}{\left(p \cdot q_{2}\right)} i \epsilon^{\mu \nu \rho \eta} q_{2 \rho} p_{\eta}\right] \bar{u}\left(p_{2}, s_{2}\right) d_{2} \gamma_{5} u\left(p_{1}, s_{1}\right) \\
\left.+\tilde{E}_{f}^{+}(x, \xi, t)\left[\frac{1}{\left(p \cdot q_{2}\right)} i \epsilon^{\mu \nu \rho \eta} q_{2 \rho} p_{\eta}\right] \frac{\left(q_{2} \cdot r\right)}{2 M} \bar{u}\left(p_{2}, s_{2}\right) \gamma_{5} u\left(p_{1}, s_{1}\right)\right\} .
\end{array}
$$


The amplitude has both real and imaginary parts. Since OFPDs are real functions, the imaginary part of the amplitude comes only from the singularity of the expression $1 /(x-\xi+i 0)$. The latter can be calculated by applying the formula

$$
\frac{1}{(x \pm i 0)} \equiv P\left(\frac{1}{x}\right) \mp i \pi \delta(x),
$$

where $P$ denotes the principal value. Thus the real part of $\mathcal{T}_{E M}^{\mu \nu}$ is obtained using the principal value prescription. The imaginary part, on the other hand, generates the delta function $\delta(x-\xi)$, which assures taking OFPDs at the specific point, $x=\xi$. In other words, the imaginary part of the amplitude is directly proportional to OFPDs evaluated along the line $x=\xi$. Moreover, from the tensor structure of the VCA in Eq. (102), it is easy to see that the amplitude exactly satisfies the electromagnetic gauge invariance with respect to the final real photon,

$$
q_{2 \mu} \mathcal{T}_{E M t w i s t-2}^{\mu \nu}=0
$$

The gauge invariant condition with respect to the initial virtual photon that has the momentum $q_{1 \nu}=q_{2 \nu}-r_{\nu}$ will only be satisfied if $\mathcal{T}_{E M t w i s t-2}^{\mu \nu} r_{\nu}=0$. However, for the part of $\mathcal{T}_{E M t w i s t-2}^{\mu \nu}$, which is symmetric with respect to $\mu \leftrightarrow \nu$, we find with the help of Eqs. (84) and (85),

$$
\begin{aligned}
\mathcal{T}_{E M t w i s t-2(s y m)}^{\mu \nu} r_{\nu} & \sim r^{\mu}-\frac{\left(r \cdot q_{2}\right)}{\left(p \cdot q_{2}\right)} p^{\mu} \\
& =\Delta^{\mu}-\mathcal{O}\left(r^{2}\right) p^{\mu}
\end{aligned}
$$

Since the components of $\Delta$ are all of the order $\sqrt{|t|}$, see Ref. [83], it follows that the leading-twist VCA is gauge invariant to accuracy $\mathcal{O}(\sqrt{|t|})$. This violation of electromagnetic gauge invariance is a higher-twist (i.e. twist-3) level effect.

\section{IV.3 KINEMATICS}

In the generalized DVCS process,

$$
l_{1}(k)+N_{1}\left(p_{1}\right) \longrightarrow l_{2}\left(k^{\prime}\right)+N_{2}\left(p_{2}\right)+\gamma\left(q_{2}\right)
$$

there are a lepton $l_{1}$ with the four-momentum $k$ and a nucleon $N_{1}\left(p_{1}\right)$ in the initial state, and a lepton $l_{2}\left(k^{\prime}\right)$, a nucleon $N_{2}\left(p_{2}\right)$ and a real photon $\gamma\left(q_{2}\right)$ in a final state. The process has two contributions that are represented by two types of diagrams, see Fig. 11. The DVCS diagram corresponds to the emission of the real photon from 
the nucleon blob. In the Bethe-Heitler diagram, the real photon is emitted from a lepton leg. We denote the four-momenta as $k=(\omega, \vec{k}), p_{1}=(M, \overrightarrow{0}), k^{\prime}=\left(\omega^{\prime}, \vec{k}^{\prime}\right)$, $p_{2}=\left(E_{2}, \vec{p}_{2}\right)$ and $q_{2}=\left(\nu_{2}, \vec{q}_{2}\right)$. The differential cross section for lepton scattering off a nucleon, to produce a final state with a lepton, nucleon and a real photon is

$$
d \sigma=\frac{1}{2 s}|\mathrm{~T}|^{2} \frac{1}{(2 \pi)^{5}} \delta^{(4)}\left(k+p_{1}-k^{\prime}-p_{2}-q_{2}\right) \frac{d^{3} k^{\prime}}{2 \omega^{\prime}} \frac{d^{3} p_{2}}{2 E_{2}} \frac{d^{3} q_{2}}{2 \nu_{2}},
$$

where $\mathrm{T}$ represents the scattering amplitude. It contains both the Compton and Bethe-Heitler contributions,

$$
\mathrm{T}=\mathrm{T}_{C}+\mathrm{T}_{B H}
$$

In the laboratory frame, as the target rest frame, the total lepton-nucleon center-ofmass energy squared is $s \equiv\left(k+p_{1}\right)^{2}=2 \omega M+M^{2}$. Note that in the following, we neglect both lepton masses. Integrating Eq. (107) over the photon momentum gives

$$
d \sigma=\frac{1}{2 s}|\mathrm{~T}|^{2} \frac{1}{(2 \pi)^{5}} \delta\left[\left(k+p_{1}-k^{\prime}-p_{2}\right)^{2}\right] \frac{\omega^{\prime} d \omega^{\prime} d \Omega^{\prime}}{2} \frac{d^{3} p_{2}}{2 E_{2}}
$$

The delta function in the cross section (109) provides the constraint $\hat{s}+M^{2}-$ $2\left[\left(\nu_{1}+M\right) E_{2}-\vec{q}_{1} \cdot \vec{p}_{2}\right]=0$, where the invariant $\hat{s} \equiv\left(p_{1}+q_{1}\right)^{2}$ and $q_{1}=k-k^{\prime}=$ $\left(\nu_{1}, \vec{q}_{1}\right)$ is the four-momentum of the virtual photon. Furthermore, we choose the coordinate system so that the $z$-axis is in the direction of the incident lepton in the plane formed by the lepton momenta. After integrating Eq. (109) over the magnitude of $\vec{p}_{2}$, the differential cross section reads

$$
d \sigma=\frac{1}{16 s(2 \pi)^{5}} \frac{\omega^{\prime}\left|\vec{p}_{2}\right|^{2}}{\left|\left(\nu_{1}+M\right)\right| \vec{p}_{2}|-| \vec{q}_{1}\left|E_{2} \cos \phi_{12}\right|}|\mathrm{T}|^{2} d \omega^{\prime} d \Omega^{\prime} d \Omega_{2},
$$

where $\phi_{12}$ is the angle between vectors $\vec{q}_{1}$ and $\vec{p}_{2}$.

Alternatively, one can choose the coordinate system depicted in Fig. 13, in which the virtual photon four-momentum has no transverse components, $q_{1}=\left(\nu_{1}, 0,0,\left|\vec{q}_{1}\right|\right)$, and the incoming and outgoing lepton four-momenta are $k=\omega(1, \sin \phi, 0, \cos \phi)$ and $k^{\prime}=\omega^{\prime}\left(1, \sin \phi^{\prime}, 0, \cos \phi^{\prime}\right)$, respectively. In this reference frame, the azimuthal angle of the recoiled nucleon corresponds to the angle $\varphi$ between the lepton and nucleon scattering planes. Using now the delta function $\delta\left[\left(k+p_{1}-k^{\prime}-p_{2}\right)^{2}\right]$ in Eq. (109) to integrate over the polar angle $\phi_{2}$ of the outgoing nucleon, we find

$$
\cos \phi_{2}=\frac{2 E_{2}\left(\omega-\omega^{\prime}\right)-q_{1}^{2}\left(1-1 / x_{B}\right)-t}{2 \sqrt{E_{2}^{2}-M^{2}} \sqrt{\left(\omega-\omega^{\prime}\right)^{2}-q_{1}^{2}}},
$$




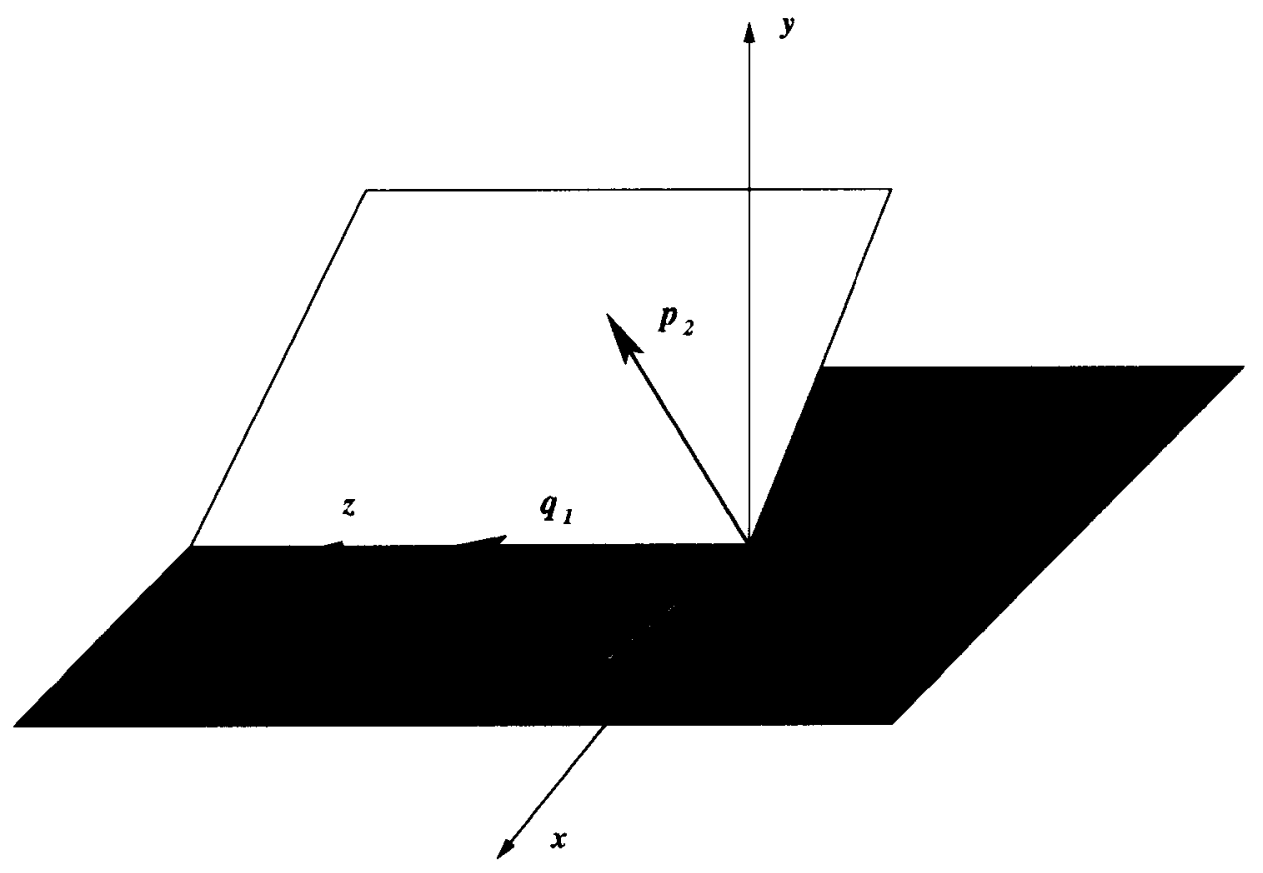

FIG. 13: Kinematics of the generalized DVCS process in the target rest frame.

and the cross section assumes the form

$$
d \sigma=\frac{1}{16 s}|\mathrm{~T}|^{2} \frac{1}{(2 \pi)^{4}} \frac{\omega^{\prime}}{\sqrt{\left(\omega-\omega^{\prime}\right)^{2}-q_{1}^{2}}} d \omega^{\prime} d E_{2} d\left(\cos \phi^{\prime}\right) d \varphi
$$

with the angle $\phi^{\prime}$ denoting the polar angle of the scattered lepton.

Instead of the kinematical variables $\omega^{\prime}, E_{2}$ and $\cos \phi^{\prime}$, it is convenient to express the differential cross section in terms of the invariant variables, e.g. $\left(y, t, x_{B}\right)$ or $\left(Q_{1}^{2}, t, x_{B}\right)$, where $Q_{1}^{2} \equiv-q_{1}^{2}$ and $y \equiv\left(p_{1} \cdot q_{1}\right) /\left(p_{1} \cdot k\right)$. In the laboratory frame, these invariants are given by

$$
\begin{aligned}
Q_{1}^{2} & =2 M \omega y x_{B}, \\
y & =\frac{\omega-\omega^{\prime}}{\omega} \\
t & =2 M^{2}-2 M E_{2} .
\end{aligned}
$$

The last invariant can also be written as $t \equiv\left(q_{1}-q_{2}\right)^{2}=-Q_{1}^{2}-$ $2 \nu_{2}\left(\omega y-\sqrt{\omega^{2} y^{2}+Q_{1}^{2}} \cos \theta_{\gamma \gamma}\right)$, where the energy of the outgoing real photon is given by the energy conservation, $\nu_{2}=M+\omega y-E_{2}$. By combining both expressions for $t$, we can express it as a function of the angle $\theta_{\gamma \gamma}$ between the incoming virtual and 
outgoing real photon, namely,

$$
t=2 M^{2}-M \frac{2 M^{2}+Q_{1}^{2}+2(M+\omega y)\left(\omega y-\sqrt{\omega^{2} y^{2}+Q_{1}^{2}} \cos \theta_{\gamma \gamma}\right)}{M+\omega y-\sqrt{\omega^{2} y^{2}+Q_{1}^{2}} \cos \theta_{\gamma \gamma}} .
$$

Moreover, from the three-momentum conservation, $\vec{q}_{1}=\vec{k}-\vec{k}^{\prime}$, and alternative expression for the invariant $Q_{1}^{2}$ in the laboratory frame, $Q_{1}^{2}=-\left(k-k^{\prime}\right)^{2}=$ $2 \omega \omega^{\prime}\left(1-\cos \phi_{l e p}\right)$, we get for the polar angles of the incoming and scattered leptons,

$$
\cos \phi=\frac{1}{\omega}\left(\sqrt{\omega^{2} y^{2}+Q_{1}^{2}}+\omega(1-y) \cos \phi^{\prime}\right)
$$

and

$$
\cos \phi^{\prime}=\frac{2 \omega^{2} y(1-y)-Q_{1}^{2}}{2 \omega(1-y) \sqrt{\omega^{2} y^{2}+Q_{1}^{2}}}
$$

respectively. The Jacobian is then equal to

$$
J \equiv\left|\frac{\partial\left(\omega^{\prime}, E_{2}, \cos \phi^{\prime}\right)}{\partial\left(y, t, x_{B}\right)}\right|=\frac{\omega^{2} y^{2}\left(\omega+M x_{B}\right)}{2(1-y)\left(\omega^{2} y^{2}+Q_{1}^{2}\right)^{3 / 2}}
$$

and accordingly, the differential cross section turns into

$$
\begin{aligned}
\frac{d^{4} \sigma}{d x_{B} d y d t d \varphi} & =\frac{d^{4} \sigma}{d\left(\cos \phi^{\prime}\right) d \omega^{\prime} d E_{2} d \varphi} J \\
& =\frac{1}{32 s} \frac{1}{(2 \pi)^{4}} \frac{1+x_{B}(M / \omega)}{\left[y+2 x_{B}(M / \omega)\right]^{2}}|T|^{2} .
\end{aligned}
$$

Note that using the invariant $Q_{1}^{2}$ instead of $y$ yields

$$
\frac{d^{4} \sigma}{d x_{B} d Q_{1}^{2} d t d \varphi}=\frac{1}{64 s} \frac{1}{(2 \pi)^{4}} \frac{1+x_{B}(M / \omega)}{M \omega x_{B}\left[y+2 x_{B}(M / \omega)\right]^{2}}|T|^{2} .
$$

In the following, we determine the kinematically allowed region for the generalized DVCS process. In other words, one needs to find the upper and lower limits on $x_{B}$, $y$ and $t$. We require the following constraints:

- The energy of the incoming lepton beam is fixed. In particular, we consider two examples that are both relevant to Jefferson Lab, namely, $\omega=5.75 \mathrm{GeV}$ and $\omega=11 \mathrm{GeV}$.

- The invariant mass of the virtual photon-nucleon system should be above the resonance region, $\hat{s} \equiv\left(p_{1}+q_{1}\right)^{2} \geq \hat{s}_{\text {min }}=4 \mathrm{GeV}^{2}$. 
- The virtuality of the incoming photon has to be large enough to secure the light-cone dominance, $Q_{1}^{2} \geq Q_{1 \min }^{2}=2.5 \mathrm{GeV}^{2}$.

- The momentum transfer squared to the nucleon should be kept as small as possible, $t / q^{2} \ll 1$.

The second constraint, $M^{2}-Q_{1}^{2}\left(1-1 / x_{B}\right) \geq \hat{s}_{\text {min }}$, together with $Q_{1}^{2}=x_{B} y\left(s-M^{2}\right)$ implies that

$$
y_{\min }=\frac{\hat{s}_{\min }-M^{2}}{\left(s-M^{2}\right)\left(1-x_{B}\right)} .
$$

On the other hand, the scaling variable $y$ reaches its maximum value,

$$
y_{\max }=\left(1+\frac{M^{2} x_{B}}{s-M^{2}}\right)^{-1},
$$

when the incoming lepton is aligned along the $z$-axis at the angle $\phi_{l e p}=180^{\circ}$. If one plots the region in the $x_{B} y$ plane, as illustrated in Fig. 14, then Eqs. (120), (121) and $y=Q_{1 m i n}^{2} / x_{B}\left(s-M^{2}\right)$ (the latter comes from the third constraint) correspond to its boundaries. Next, both lower and upper limits of the invariant $t$ can be found if we go in the virtual photon-nucleon center-of-mass frame. In this particular frame, at the scattering angles between the initial and final nucleons equal to $0^{\circ}$ and $180^{\circ}$, the invariant momentum attains, up to relative corrections of the order $x_{B} M^{2} / Q_{1}^{2}$, its kinematical limits,

$$
\begin{aligned}
t_{\text {min }} & =\frac{-M^{2} x_{B}^{2}}{1-x_{B}\left(1-M^{2} / Q_{1}^{2}\right)} \\
t_{\max } & =\frac{M^{2} x_{B}^{2}-2 M^{2} x_{B}+Q_{1}^{2}\left(x_{B}-1\right) / x_{B}}{1-x_{B}\left(1-M^{2} / Q_{1}^{2}\right)} .
\end{aligned}
$$

They are presented, within the kinematically allowed regions, in Table I. Since we require small $t$, the upper limit becomes irrelevant. In addition, having small $t$ yields a low-energy nucleon with $E_{2}=M\left(1-t / 2 M^{2}\right)$ and a high-energy real photon in the final state.

Finally, for each lepton energy we pick one kinematical point within the allowed region in the $x_{B} y$ plane. In particular, we take $Q_{1}^{2}=2.5 \mathrm{GeV}^{2}$ for the virtuality of the initial photon and $x_{B}=0.35$. In Fig. 15, the invariant $t$ is plotted against the angle $\theta_{\gamma \gamma}$. It is customary to present the plot for the values of $t$ up to $-1 \mathrm{GeV}^{2}$, which happens at $\theta_{\gamma \gamma} \simeq 12^{0}$, even though we require that, in principle, $-t$ should be much smaller than $Q_{1}^{2}$. The values of $t$ vary from $-0.15 \mathrm{GeV}^{2}$ to $-1.433 \mathrm{GeV}^{2}$ for angles 


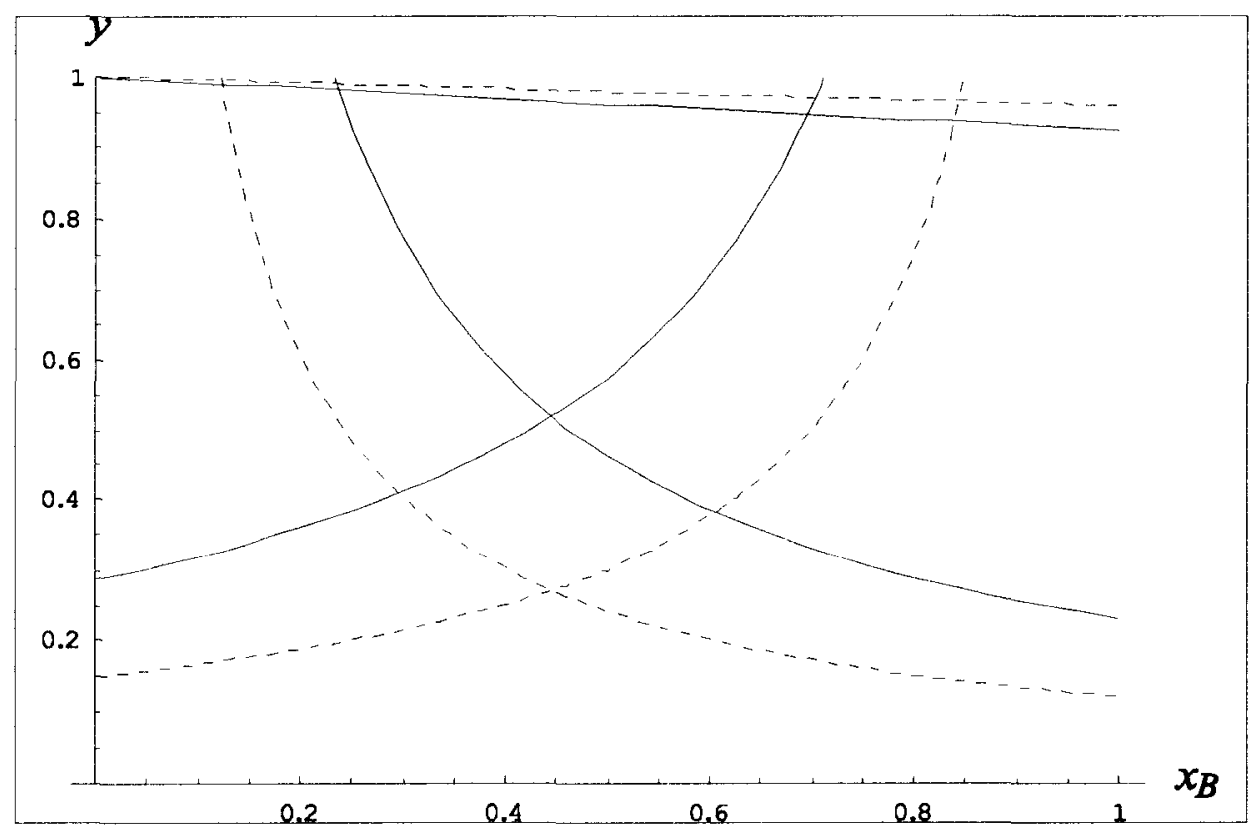

FIG. 14: Kinematically allowed region for $\hat{s} \geq 4 \mathrm{GeV}^{2}$ and $Q_{1}^{2} \geq 2.5 \mathrm{GeV}^{2}$ with $\omega=5.75 \mathrm{GeV}$ (solid line) and $\omega=11 \mathrm{GeV}$ (dashed line) lepton beam.

TABLE I: Lower and upper limits of the invariant momentum transfer $t$ within the kinematically allowed region illustrated in Fig. 14 for two different lepton beam energies $\omega$.

\begin{tabular}{lcc}
\hline \hline$\omega[\mathrm{GeV}]$ & $-t_{\min }\left[\mathrm{GeV}^{2}\right]$ & $-t_{\max }\left[\mathrm{GeV}^{2}\right]$ \\
\hline 5.75 & 0.058 & 10.019 \\
11 & 0.014 & 19.853 \\
\hline \hline
\end{tabular}




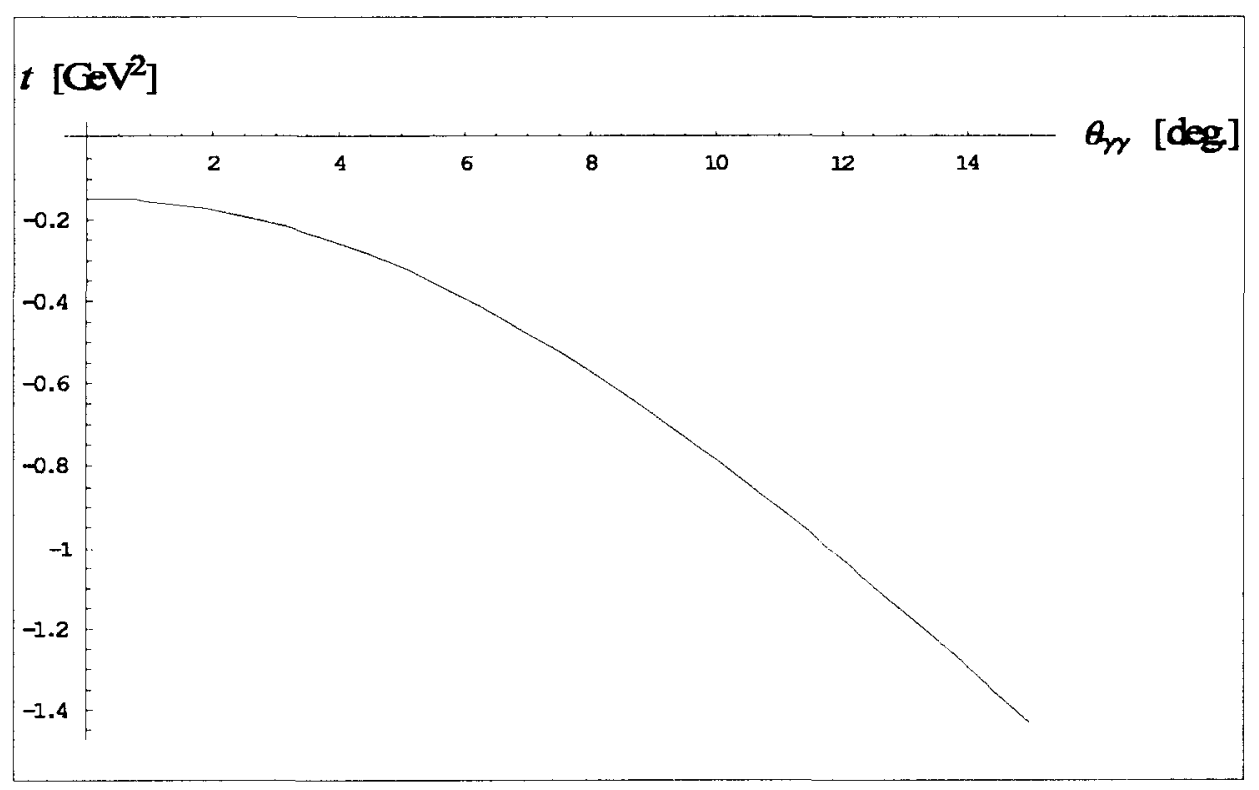

FIG. 15: Invariant momentum transfer $t$ plotted as a function of the angle $\theta_{\gamma \gamma}$ between the incoming virtual and outgoing real photon in the target rest frame for $Q_{1}^{2}=$ $2.5 \mathrm{GeV}^{2}$ and $x_{B}=0.35$.

TABLE II: Polar angles $\phi$ and $\phi^{\prime}$ of the incoming and scattered leptons, respectively, in the target rest frame for $Q_{1}^{2}=2.5 \mathrm{GeV}^{2}$ and $x_{B}=0.35$ with two different lepton beam energies $\omega$.

\begin{tabular}{lcc}
\hline \hline$\omega[\mathrm{GeV}]$ & $\phi[\mathrm{deg}]$. & $\phi^{\prime}[\mathrm{deg}]$. \\
\hline 5.75 & 12.6 & 39.9 \\
11 & 18 & 28.2 \\
\hline \hline
\end{tabular}


between $0 \leq \theta_{\gamma \gamma} \leq 15^{0}$. Note that by fixing $Q_{1}^{2}$ and $x_{B}$, the product $\omega y=Q_{1}^{2} / 2 M x_{B}$, which appears in Eq. (114) is also fixed and accordingly, we end up with the same $\theta_{\gamma \gamma}$-dependence for both beam energies. For convenience, we set the angle between the lepton and nucleon scattering planes to $\varphi=0$, and arrive at the so-called in-plane kinematics. The polar angles of both incoming and scattered leptons are then fixed and given in Table II.

\section{IV.4 TOY MODEL}

Our simple model has the following properties:

- We assume that the sea quark contribution is negligible. For that reason, the plus OFPDs in the parametrization (96) are equal to the valence OFPDs, $H_{f}^{+}=$ $H_{f}^{v a l}$ and $\tilde{H}_{f}^{+}=\tilde{H}_{f}^{v a l}$, with the quark flavor $f=u, d$, and similarly for $E_{f}^{+}$and $\tilde{E}_{f}^{+}$distributions.

- We assume that for all distributions the $t$-dependence factorize from the dependence on other two scaling variables. The dependence of GPDs on the invariant $t$ is then characterized by the corresponding form factors.

- The $\xi$-dependence of OFPDs appears only in the $\tilde{E}_{f}^{+}$distribution.

The parametrization of the unpolarized quark OFPDs is taken from Ref. [92]. Namely,

$$
\begin{aligned}
H_{u}^{v a l}(x, \xi, t) & =u_{N}^{v a l}(x) F_{1 u}(t) / 2 \\
H_{d}^{v a l}(x, \xi, t) & =d_{N}^{\text {val }}(x) F_{1 d}(t) \\
E_{u}^{v a l}(x, \xi, t) & =u_{N}^{v a l}(x) F_{2 u}(t) / 2 \\
E_{d}^{v a l}(x, \xi, t) & =d_{N}^{v a l}(x) F_{2 d}(t)
\end{aligned}
$$

In particular, for the proton target the unpolarized valence quark distributions in the proton are given by [38]

$$
\begin{aligned}
& u_{p}^{v a l}(x)=1.89 x^{-0.4}(1-x)^{3.5}(1+6 x) \\
& d_{p}^{v a l}(x)=0.54 x^{-0.6}(1-x)^{4.2}(1+8 x)
\end{aligned}
$$

They closely reproduce corresponding GRV parametrizations [93] at a low normalization point, $-q_{1}^{2} \simeq 1 \mathrm{GeV}^{2}[94]$. 
The $u$ - and $d$-quark form factors in Eq. (123) can be extracted from the proton and neutron Dirac and Pauli form factors according to $F_{1 p(n)}=Q_{u} F_{1 u(d)}+Q_{d} F_{1 d(u)}$ and $F_{2 p(n)}=Q_{u} F_{2 u(d)}+Q_{d} F_{2 d(u)}$. Furthermore, the proton and neutron form factors are related to the Sachs electric and magnetic form factors, see Eqs. (54) and (55), through

$$
\begin{aligned}
& F_{1 p(n)}(t)=\left[G_{E p(n)}(t)-\frac{t}{4 M^{2}} G_{M p(n)}(t)\right]\left(1-\frac{t}{4 M^{2}}\right)^{-1}, \\
& F_{2 p(n)}(t)=\left[G_{M p(n)}(t)-G_{E p(n)}(t)\right]\left(1-\frac{t}{4 M^{2}}\right)^{-1},
\end{aligned}
$$

where the nucleon mass is $M \simeq 0.94 \mathrm{GeV}$. In the region of small $t$, both Sachs form factors are well described by a dipole fit,

$$
G_{E p}(t)=\frac{G_{M p}(t)}{1+\kappa_{p}}=\frac{G_{M n}(t)}{\kappa_{n}}=\left(1-\frac{t}{\Lambda^{2}}\right)^{-2} \text { and } G_{E n}(t)=0,
$$

with the parameter $\Lambda^{2}=0.71 \mathrm{GeV}^{2}$ and $\kappa_{p}=1.793$ and $\kappa_{n}=-1.913$ are the proton and neutron anomalous magnetic moments, respectively. In the polarized case, we take for the valence distributions [95]

$$
\begin{aligned}
& \tilde{H}_{u}^{v a l}(x, \xi, t)=\Delta u_{p}^{v a l}(x)\left(1-\frac{t}{m_{A}^{2}}\right)^{-2}, \\
& \tilde{H}_{d}^{v a l}(x, \xi, t)=\Delta d_{p}^{v a l}(x)\left(1-\frac{t}{m_{A}^{2}}\right)^{-2},
\end{aligned}
$$

with the mass parameter $m_{A}=1.03 \mathrm{GeV}$. The $t$-dependence in Eq. (127) corresponds to the ratio $g_{A}(t) / g_{A}(t=0)$. The polarized valence quark distributions in the proton can be expressed in terms of the unpolarized distributions in the following way [96]

$$
\begin{aligned}
& \Delta u_{p}^{v a l}=\cos \theta_{D}\left(u_{p}^{v a l}-\frac{2}{3} d_{p}^{v a l}\right) \\
& \Delta d_{p}^{v a l}=\cos \theta_{D}\left(-\frac{1}{3} d_{p}^{\text {val }}\right)
\end{aligned}
$$

where $\cos \theta_{D}=\left[1+\mathrm{H}_{0}\left(1-x^{2}\right) / \sqrt{x}\right]^{-1}$ with $\mathrm{H}_{0}=0.06$. Finally, for the $\tilde{E}_{f}$ distribution we accept the pion pole dominated ansatz,

$$
\begin{aligned}
\tilde{E}_{u}^{v a l}(x, \xi, t) & =\frac{1}{2} F_{\pi}(t) \frac{\theta(|x|<\xi)}{2 \xi} \phi_{\pi}\left(\frac{x+\xi}{2 \xi}\right) \\
\tilde{E}_{d}^{v a l}(x, \xi, t) & =-\tilde{E}_{u}^{v a l}(x, \xi, t) .
\end{aligned}
$$

The function $F_{\pi}(t)$ is taken in the form valid for $-t \ll M^{2}[97]$,

$$
F_{\pi}(t)=4 g_{A}(t=0) M^{2}\left[\frac{1}{\left(m_{\pi}^{2}-t\right) / \mathrm{GeV}^{2}}-\frac{1.7}{\left(1-t / 2 \mathrm{GeV}^{2}\right)^{2}}\right],
$$


where $m_{\pi} \simeq 0.14 \mathrm{GeV}$ denotes the pion mass and $g_{A}(t=0)=1.267$. For the pion distribution amplitude in Eq. (129) we choose, for simplicity, its asymptotic form

$$
\phi_{\pi}(u)=6 u(1-u)
$$

\section{IV.5 CROSS SECTION}

Having defined the kinematics and described the simple model, in the following we compute separately the unpolarized cross sections for the Compton and Bethe-Heitler contributions to the DVCS process on a proton target using an electron beam. In fact, since the scattering amplitude consists of two parts, see Eq. (108), its modulus squared (and, accordingly the cross section) is given by the sum of three terms, namely, the Compton term, the Bethe-Heitler term and the interference term. Here we only consider the first two terms. Their cross sections are plotted for the in-plane kinematics against the angle $\theta_{\gamma \gamma}$ by taking the same kinematical point, $Q_{1}^{2}=2.5 \mathrm{GeV}^{2}$ and $x_{B}=0.35$, however, for two beam energies, $\omega=5.75 \mathrm{GeV}$ and $11 \mathrm{GeV}$.

With the help of the momentum-space Feynman rules for QED we can immediately write down the T-matrix for the pure Compton process. Denoting the polarization vector of the final real photon by $\epsilon_{\mu}^{*}\left(q_{2}\right)$, we have

$$
i \mathrm{~T}_{C}=\bar{u}\left(k^{\prime}\right)\left(i|e| \gamma^{\lambda}\right) u(k)\left(\frac{-i g_{\nu \lambda}}{q_{1}^{2}}\right)\left(-e^{2}\right) \epsilon_{\mu}^{*}\left(q_{2}\right)\left(-i \mathcal{T}_{E M}^{\mu \nu}\right)
$$

or

$$
\mathrm{T}_{C}=\frac{|e|^{3}}{q_{1}^{2}} \bar{u}\left(k^{\prime}\right) \gamma_{\nu} u(k) \epsilon_{\mu}^{*}\left(q_{2}\right) \mathcal{T}_{E M}^{\mu \nu}
$$

where $\mathcal{T}_{E M}^{\mu \nu}$ is given by the expression (102). Then one should average the square of Eq. (133) over the initial proton and electron spins, and further sum it over the final proton and electron spins and photon polarizations. Note that this particular summation is performed using the Feynman gauge prescription, i.e. one can replace

$$
\sum_{\gamma \text { polar. }} \epsilon_{\mu}^{*}\left(q_{2}\right) \epsilon_{\alpha}\left(q_{2}\right) \longrightarrow-g_{\mu \alpha}
$$

by virtue of the Ward identity. As a result, we get a factorized expression,

$$
\overline{\left|\mathrm{T}_{C}\right|^{2}}=\frac{(4 \pi \alpha)^{3}}{q_{1}^{4}} L_{\nu \beta}^{C} H_{C}^{\nu \beta},
$$


in terms of the electron and hadron tensors. Neglecting the electron mass, the electron tensor simply reads

$$
L_{\nu \beta}^{C}=2\left[k_{\nu} k_{\beta}^{\prime}+k_{\beta} k_{\nu}^{\prime}-g_{\nu \beta}\left(k \cdot k^{\prime}\right)\right]
$$

The hadron tensor requires some algebra, however, if we define the convolution integrals of OFPDs as a new set of functions,

$$
\begin{aligned}
\mathcal{H}^{+}(\xi, t) & \equiv \sum_{f} Q_{f}^{2} \int_{-1}^{1} \frac{d x}{(x-\xi+i 0)} H_{f}^{+}(x, \xi, t), \\
\mathcal{E}^{+}(\xi, t) & \equiv \sum_{f} Q_{f}^{2} \int_{-1}^{1} \frac{d x}{(x-\xi+i 0)} E_{f}^{+}(x, \xi, t), \\
\tilde{\mathcal{H}}^{+}(\xi, t) & \equiv \sum_{f} Q_{f}^{2} \int_{-1}^{1} \frac{d x}{(x-\xi+i 0)} \tilde{H}_{f}^{+}(x, \xi, t), \\
\tilde{\mathcal{E}}^{+}(\xi, t) & \equiv \sum_{f} Q_{f}^{2} \int_{-1}^{1} \frac{d x}{(x-\xi+i 0)} \tilde{E}_{f}^{+}(x, \xi, t),
\end{aligned}
$$

and, in addition, neglect terms $\mathcal{O}\left(t / q_{1}^{2}\right)$ and $\mathcal{O}\left(M^{2} / q_{1}^{2}\right)$, then $H_{C}^{\nu \beta}$ can be written in a compact form as

$$
\begin{aligned}
H_{C}^{\nu \beta}= & -\frac{1}{2} \mathcal{T}_{E M}^{\mu \nu}\left(\mathcal{T}_{\mu E M}^{\beta}\right)^{*} \\
= & -\left\{\left[\left(1-\xi^{2}\right)\left(\left|\mathcal{H}^{+}\right|^{2}+\left|\tilde{\mathcal{H}}^{+}\right|^{2}\right)-\left(\xi^{2}+\frac{t}{4 M^{2}}\right)\left|\mathcal{E}^{+}\right|^{2}\right.\right. \\
& \left.-\xi^{2} \frac{t}{4 M^{2}}\left|\tilde{\mathcal{E}}^{+}\right|^{2}-2 \xi^{2} \Re\left(\mathcal{H}^{+*} \mathcal{E}^{+}+\tilde{\mathcal{H}}^{+*} \tilde{\mathcal{E}}^{+}\right)\right] \\
& \times\left[g^{\nu \beta}-\frac{1}{\left(p \cdot q_{2}\right)}\left(p^{\nu} q_{2}^{\beta}+p^{\beta} q_{2}^{\nu}\right)+\frac{M^{2}}{\left(p \cdot q_{2}\right)^{2}}\left(1-\frac{t}{4 M^{2}}\right) q_{2}^{\nu} q_{2}^{\beta}\right] .
\end{aligned}
$$

The initial overall factor of $1 / 2$ in Eq. (138) comes from averaging over the initial proton spin. Substituting now Eq. (135) into Eq. (119) gives the unpolarized differential cross section for the Compton contribution to the standard electromagnetic DVCS process

$$
\frac{d^{4} \sigma_{C}}{d x_{B} d Q_{1}^{2} d t d \varphi}=\frac{\alpha^{3}}{16 \pi Q_{1}^{4}} \frac{1+x_{B}(M / \omega)}{M^{2} \omega^{2}[2+(M / \omega)] x_{B}\left[y+2 x_{B}(M / \omega)\right]^{2}} L_{\nu \beta}^{C} H_{C}^{\nu \beta} .
$$

Its dependence on the angle $\theta_{\gamma \gamma}$ is shown in Fig. 16.

Apart from the Compton part, the Bethe-Heitler contribution can be fully calculable in QED with the knowledge on the nucleon form factors. The amplitude, which 


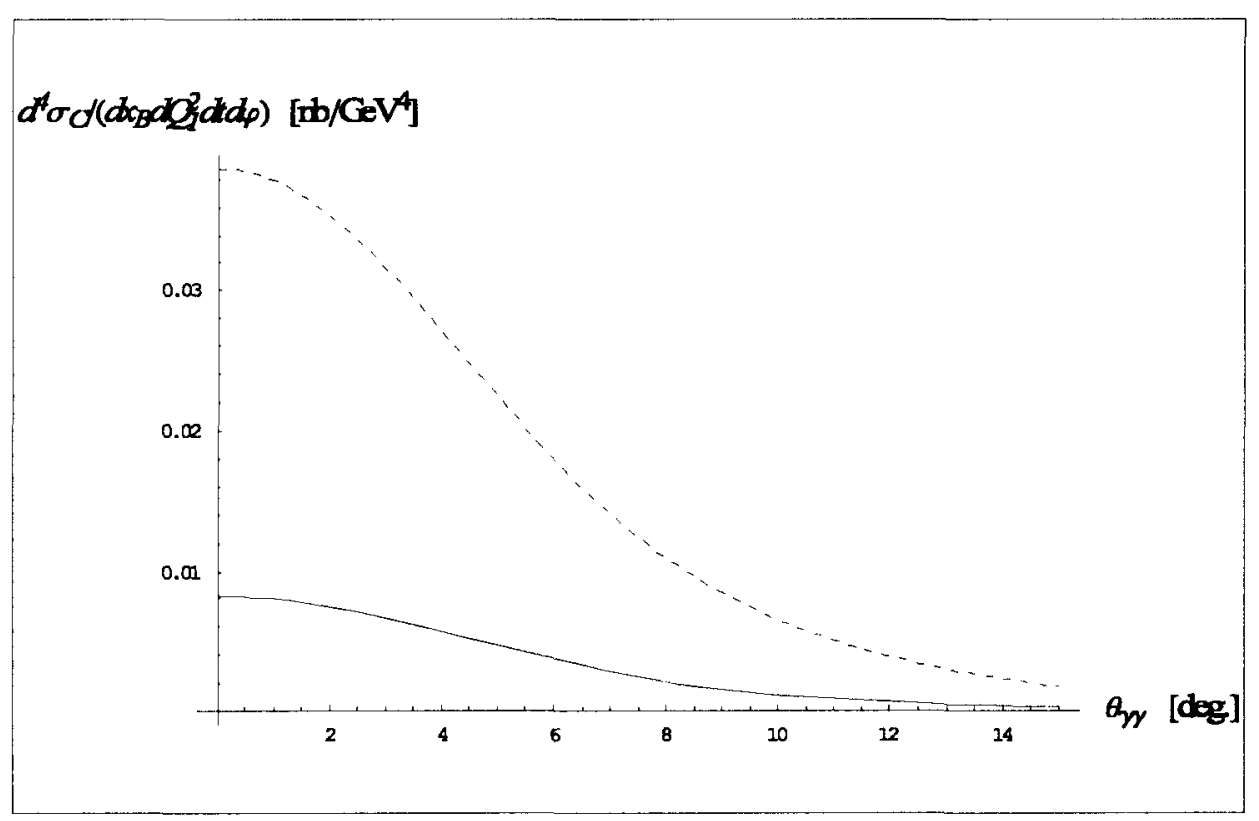

FIG. 16: Compton cross section $\sigma_{C}$ plotted as a function of the angle $\theta_{\gamma \gamma}$ between the incoming virtual and outgoing real photon in the target rest frame for $Q_{1}^{2}=2.5 \mathrm{GeV}^{2}$ and $x_{B}=0.35$ with $\omega=5.75 \mathrm{GeV}$ (solid line) and $\omega=11 \mathrm{GeV}$ (dashed line) electron beam.

in contrast to $\mathrm{T}_{C}$ is purely real, emerges from two Bethe-Heitler diagrams shown in Fig. 11,

$$
\begin{aligned}
i \mathrm{~T}_{B H}= & \bar{u}\left(k^{\prime}\right)\left(i|e| \gamma^{\mu}\right) \epsilon_{\mu}^{*}\left(q_{2}\right) \frac{i\left(\not k^{\prime}+\not q_{2}\right)}{\left(k^{\prime}+q_{2}\right)^{2}}\left(i|e| \gamma^{\nu}\right) u(k)\left(\frac{-i g_{\nu \lambda}}{\left(q_{1}-q_{2}\right)^{2}}\right) \\
& \times(-i|e|)\left\langle p\left(p_{2}, s_{2}\right)\left|J_{E M}^{\lambda}(0)\right| p\left(p_{1}, s_{1}\right)\right\rangle \\
& +\bar{u}\left(k^{\prime}\right)\left(i|e| \gamma^{\nu}\right) \frac{i\left(\not k-\not q_{2}\right)}{\left(k-q_{2}\right)^{2}}\left(i|e| \gamma^{\mu}\right) \epsilon_{\mu}^{*}\left(q_{2}\right) u(k)\left(\frac{-i g_{\nu \lambda}}{\left(q_{1}-q_{2}\right)^{2}}\right) \\
& \times(-i|e|)\left\langle p\left(p_{2}, s_{2}\right)\left|J_{E M}^{\lambda}(0)\right| p\left(p_{1}, s_{1}\right)\right\rangle .
\end{aligned}
$$

Using the relations, $\bar{u}\left(k^{\prime}\right) \gamma^{\mu} \not^{\prime}=2 \bar{u}\left(k^{\prime}\right) k^{\prime \mu}$ and $\not \gamma^{\mu} u(k)=2 k^{\mu} u(p)$, we simplify the numerators of both fermion propagators in Eq. (140), and obtain for the T-matrix

$$
\begin{aligned}
\mathrm{T}_{B H}= & \frac{|e|^{3}}{t} \epsilon_{\mu}^{*}\left(q_{2}\right) \bar{u}\left(k^{\prime}\right)\left[\frac{\gamma^{\mu} q_{2} \gamma^{\nu}+2 k^{\prime \mu} \gamma^{\nu}}{2\left(k^{\prime} \cdot q_{2}\right)}+\frac{-\gamma^{\nu} \not q_{2} \gamma^{\mu}+2 \gamma^{\nu} k^{\mu}}{-2\left(k \cdot q_{2}\right)}\right] u(k) \\
& \times\left\langle p\left(p_{2}, s_{2}\right)\left|J_{\nu}^{E M}(0)\right| p\left(p_{1}, s_{1}\right)\right\rangle .
\end{aligned}
$$

The matrix element of the proton transition current, see Section III.2, is parametrized in terms of the usual Dirac and Pauli proton form factors,

$$
\left\langle p\left(p_{2}, s_{2}\right)\left|J_{\nu}^{E M}(0)\right| p\left(p_{1}, s_{1}\right)\right\rangle=\bar{u}\left(p_{2}, s_{2}\right)\left[F_{1 p}(t) \gamma_{\nu}-F_{2 p}(t) \frac{i \sigma_{\nu \lambda} r^{\lambda}}{2 M}\right] u\left(p_{1}, s_{1}\right)
$$


The spin-averaged square of Eq. (141) is again written in terms of two tensors,

$$
\overline{\left|\mathrm{T}_{B H}\right|^{2}}=\frac{(4 \pi \alpha)^{3}}{t^{2}} L_{B H}^{\nu \beta} H_{\nu \beta}^{B H} .
$$

Here the hadron tensor reduces to a simple expression, namely,

$$
\begin{aligned}
H_{\nu \beta}^{B H}= & \frac{1}{2} \sum_{s_{1}, s_{2}}\left\langle p\left(p_{2}, s_{2}\right)\left|J_{\nu}^{E M}(0)\right| p\left(p_{1}, s_{1}\right)\right\rangle\left\langle p\left(p_{2}, s_{2}\right)\left|J_{\beta}^{E M}(0)\right| p\left(p_{1}, s_{1}\right)\right\rangle^{*} \\
= & t\left[g_{\nu \beta}-\frac{r_{\nu} r_{\beta}}{t}\right]\left[F_{1 p}(t)+F_{2 p}(t)\right]^{2} \\
& +4\left[p_{1 \nu}+\frac{r_{\nu}}{2}\right]\left[p_{1 \beta}+\frac{r_{\beta}}{2}\right]\left[F_{1 p}^{2}(t)-\frac{t}{4 M^{2}} F_{2 p}^{2}(t)\right]
\end{aligned}
$$

and the lepton tensor is

$$
\begin{aligned}
L_{B H}^{\nu \beta}= & -\frac{1}{2} \operatorname{Tr}\left\{\not k^{\prime}\left[\frac{\gamma^{\mu} q_{2} \gamma^{\nu}+2 k^{\prime \mu} \gamma^{\nu}}{2\left(k^{\prime} \cdot q_{2}\right)}+\frac{\gamma^{\nu} \not_{2} \gamma^{\mu}-2 \gamma^{\nu} k^{\mu}}{2\left(k \cdot q_{2}\right)}\right]\right. \\
& \left.\times \not k\left[\frac{\gamma^{\beta} q_{2} \gamma_{\mu}+2 \gamma^{\beta} k_{\mu}^{\prime}}{2\left(k^{\prime} \cdot q_{2}\right)}+\frac{\gamma_{\mu} q_{2} \gamma^{\beta}-2 k_{\mu} \gamma^{\beta}}{2\left(k \cdot q_{2}\right)}\right]\right\} \\
= & \frac{2}{\left(k^{\prime} \cdot q_{2}\right)}\left[k^{\nu} q_{2}^{\beta}+k^{\beta} q_{2}^{\nu}-g^{\nu \beta}\left(k \cdot q_{2}\right)\right]+\frac{2}{\left(k \cdot q_{2}\right)}\left[k^{\prime \nu} q_{2}^{\beta}+k^{\prime \beta} q_{2}^{\nu}-g^{\nu \beta}\left(k^{\prime} \cdot q_{2}\right)\right] \\
& +\frac{2}{\left(k^{\prime} \cdot q_{2}\right)\left(k \cdot q_{2}\right)}\left[\left(k \cdot q_{2}\right)\left[k^{\nu} k^{\prime \beta}+k^{\beta} k^{\prime \nu}+2 k^{\nu} k^{\prime \beta}\right]\right. \\
& -\left(k^{\prime} \cdot q_{2}\right)\left[k^{\nu} k^{\prime \beta}+k^{\beta} k^{\prime \nu}+2 k^{\nu} k^{\beta}\right] \\
& +\left(k \cdot k^{\prime}\right)\left[k^{\nu} q_{2}^{\beta}+k^{\beta} q_{2}^{\nu}-k^{\prime \nu} q_{2}^{\beta}-k^{\prime \beta} q_{2}^{\nu}+2 k^{\nu} k^{\prime \beta}+2 k^{\beta} k^{\prime \nu}\right] \\
& \left.+2 g^{\nu \beta}\left(k \cdot k^{\prime}\right)\left[\left(k^{\prime} \cdot q_{2}\right)-\left(k \cdot q_{2}\right)-\left(k \cdot k^{\prime}\right)\right]\right] .
\end{aligned}
$$

Finally, the unpolarized differential cross section reads

$$
\frac{d^{4} \sigma_{B H}}{d x_{B} d Q_{1}^{2} d t d \varphi}=\frac{\alpha^{3}}{16 \pi t^{2}} \frac{1+x_{B}(M / \omega)}{M^{2} \omega^{2}[2+(M / \omega)] x_{B}\left[y+2 x_{B}(M / \omega)\right]^{2}} L_{B H}^{\nu \beta} H_{\nu \beta}^{B H} .
$$

The Bethe-Heitler contribution is illustrated on a logarithmic scale in Figs. 17 and 18. We plot the latter for a wider range in $\theta_{\gamma \gamma}$ to be able to see both poles at the angles $\phi$ and $\phi^{\prime}$, given in Table II. The poles corresponds to the situation, when the outgoing real photon is collinear either with the incoming or scattered electron. In Fig. 19, a logarithmic plot of both contributions together is shown for $\omega=5.75 \mathrm{GeV}$. The comparison of the results shows that the Bethe-Heitler signal is well above the 


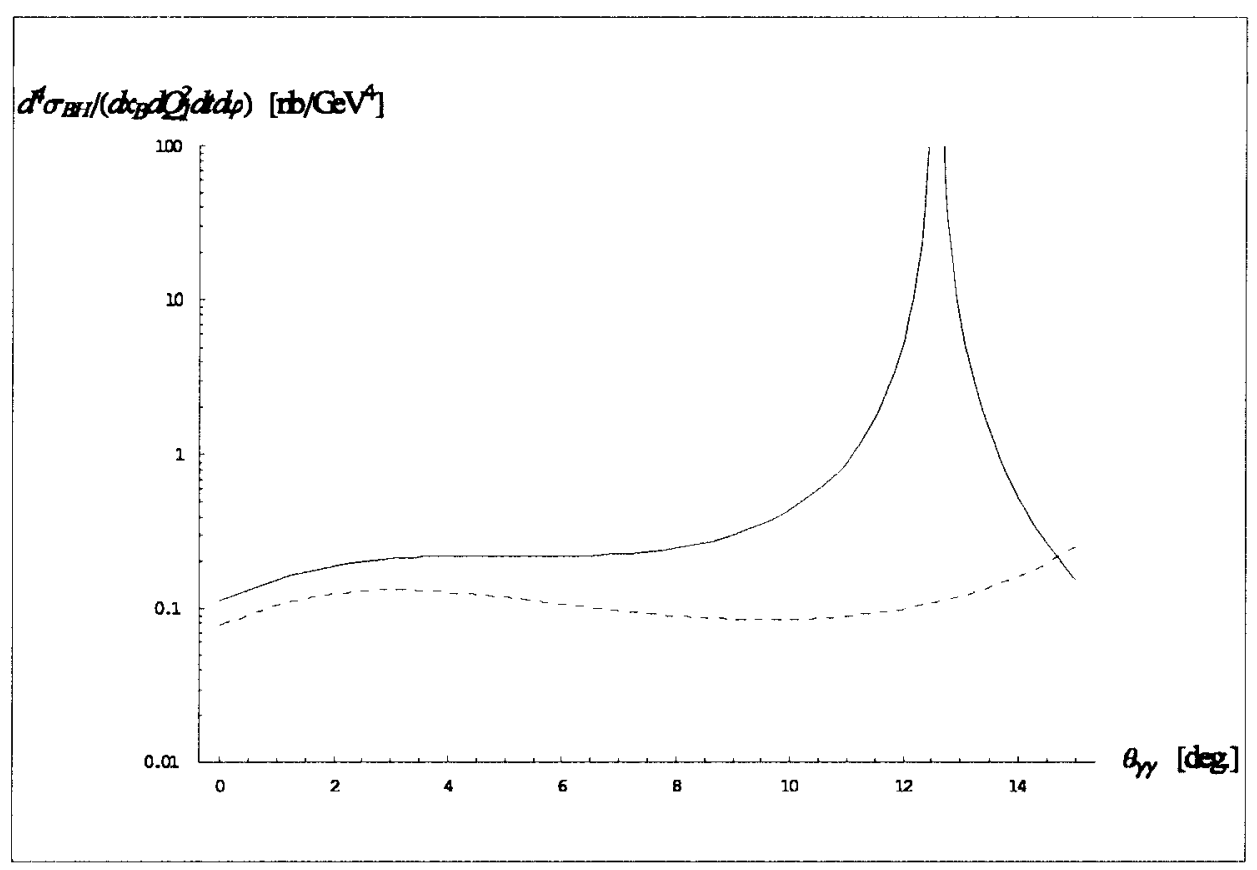

FIG. 17: Bethe-Heitler cross section $\sigma_{B H}$ plotted as a function of the angle $\theta_{\gamma \gamma}$ between the incoming virtual and outgoing real photon in the target rest frame for $Q_{1}^{2}=$ $2.5 \mathrm{GeV}^{2}$ and $x_{B}=0.35$ with $\omega=5.75 \mathrm{GeV}$ (solid line) and $\omega=11 \mathrm{GeV}$ (dashed line) electron beam.

Compton one. This is easy to see just by comparing the factorized expressions (135) and (143), and recalling that $t \ll q_{1}^{2}$. The presence of $1 / t^{2}$ in the Bethe-Heitler part enhances its contribution with respect to the Compton part, which is proportional to $1 / q_{1}^{4}$.

One way to minimize the contamination with the dominating Bethe-Heitler process is to find the kinematical regions, where the Bethe-Heitler contribution is suppressed, or at least comparable with the Compton contribution. Another way is to exploit the interference between the two processes. This approach is based on the fact that $\mathrm{T}_{C}$ has both real and imaginary parts while $\mathrm{T}_{B H}$ is purely real. By incorporating the interference terms between the Compton and Bethe-Heitler amplitudes, we can disentangle $\Re \mathrm{T}_{C}$ and $\Im \mathrm{T}_{C}$. In particular, using the positron beam in addition to the electron beam, one can measure the so-called beam-charge asymmetry, which is sensitive to the real part of $\mathrm{T}_{C}$. Furthermore, measuring the single-spin (or alternatively the beam-spin asymmetry) by considering electrons with opposite helicities gives access to the imaginary part of $\mathrm{T}_{C}$. First experiments of this kind were performed at Jefferson Lab [98] and at Hermes [99]. As a result, we can project out independently 


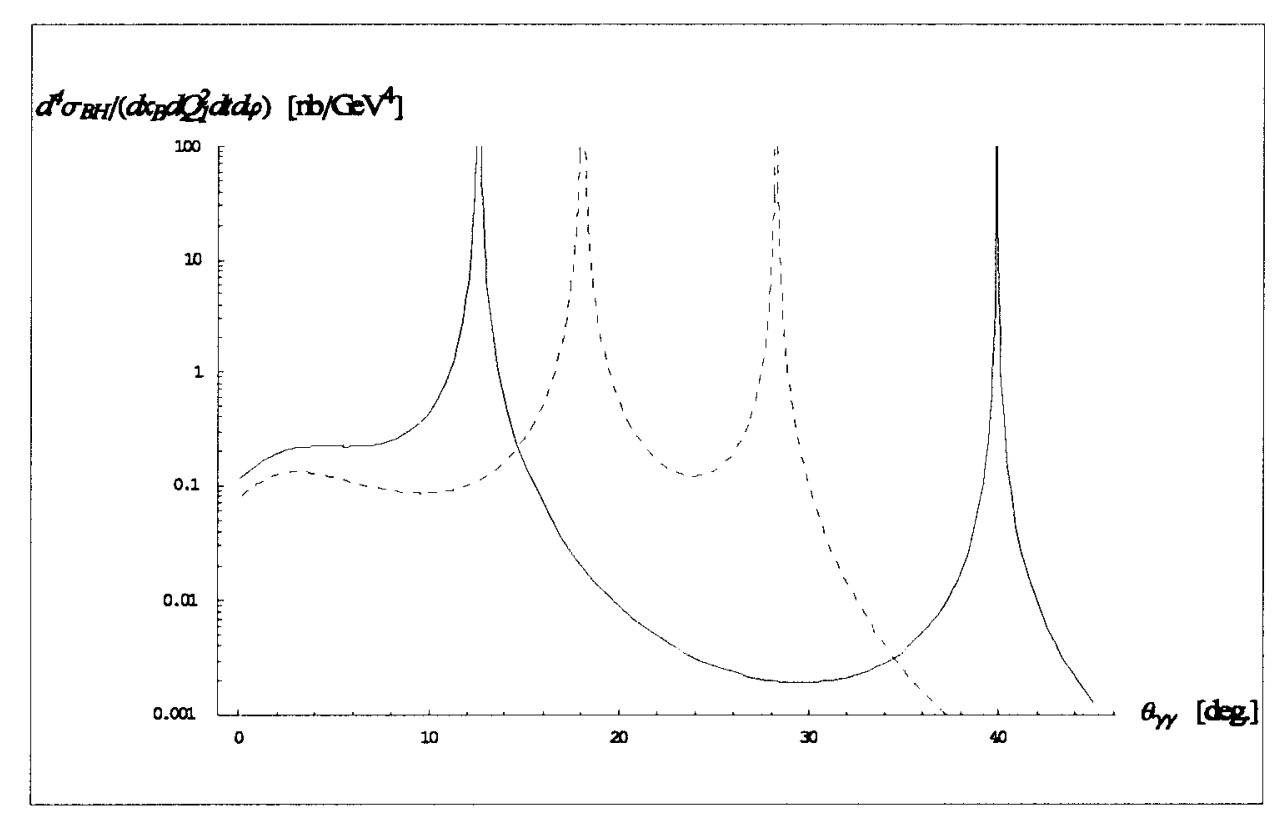

FIG. 18: Bethe-Heitler cross section $\sigma_{B H}$ plotted as a function of the angle $\theta_{\gamma \gamma}$ between the incoming virtual and outgoing real photon in the target rest frame for $Q_{1}^{2}=$ $2.5 \mathrm{GeV}^{2}$ and $x_{B}=0.35$ with $\omega=5.75 \mathrm{GeV}$ (solid line) and $\omega=11 \mathrm{GeV}$ (dashed line) electron beam.

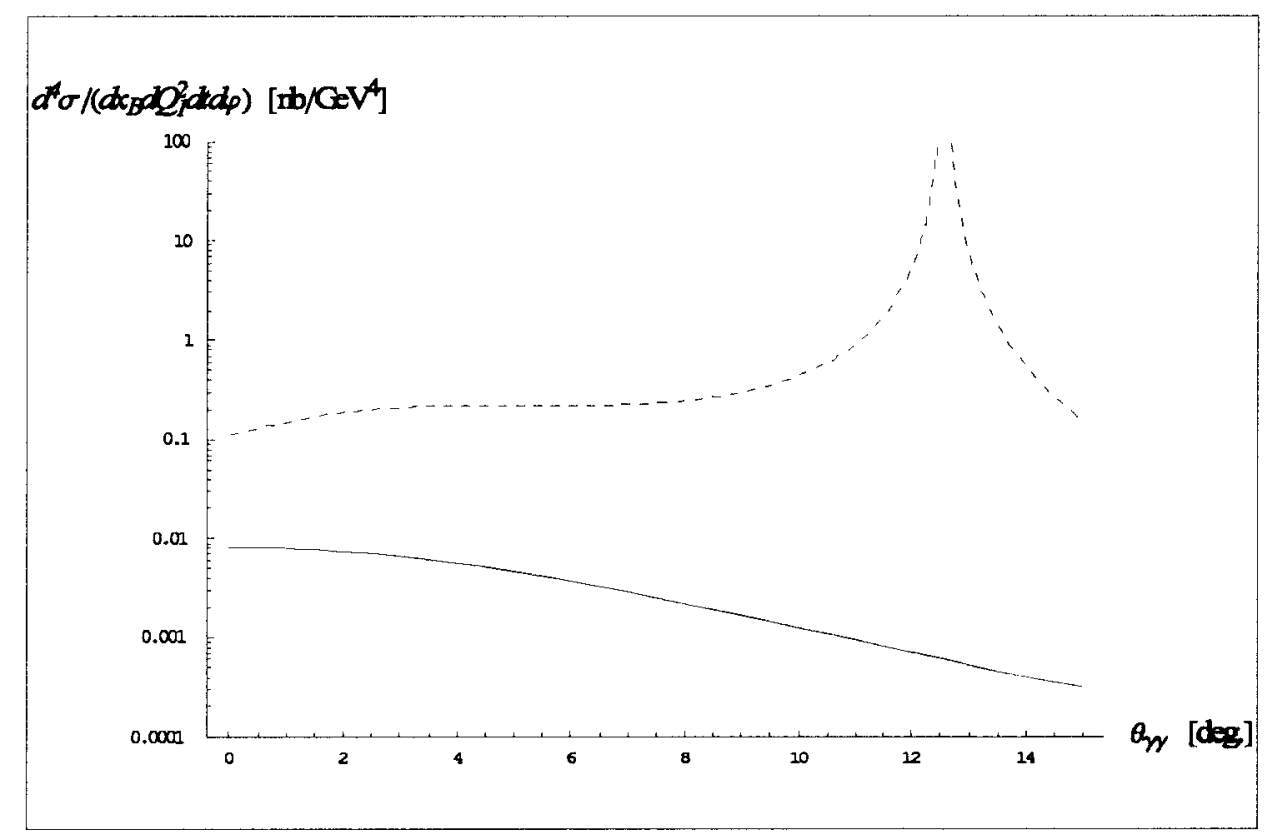

FIG. 19: Compton cross section $\sigma_{C}$ (solid line) and Bethe-Heitler cross section $\sigma_{B H}$ (dashed line) plotted as a function of the angle $\theta_{\gamma \gamma}$ between the incoming virtual and outgoing real photon in the target rest frame for $Q_{1}^{2}=2.5 \mathrm{GeV}^{2}$ and $x_{B}=0.35$ with $\omega=5.75 \mathrm{GeV}$ electron beam. 
both parts of the amplitude and accordingly, probe different linear combinations of OFPDs. 


\section{CHAPTER V}

\section{INCLUSIVE PHOTOPRODUCTION OF LEPTON PAIRS}

\section{V.1 INTRODUCTION}

By the inclusive photoproduction of lepton pairs, see Ref. [100], we refer to the reaction, in which a high-energy real photon $\gamma$ (with the four-momentum $q$ ) scatters inelastically from a nucleon $N(P)$ emitting a pair of leptons (electrons or muons) with momenta $k$ and $k^{\prime}$,

$$
\gamma(q)+N(P) \longrightarrow l^{-}(k)+l^{+}\left(k^{\prime}\right)+X .
$$

The process is shown in Fig. 20, where again $X$ labels a system of hadrons produced through inelastic processes. The reaction (147) is a crossed channel to inclusive virtual Compton scattering,

$$
e^{-}+N \longrightarrow e^{-}+\gamma+X
$$

which was originally studied in the parton model in Ref. [101].

In the framework of the QCD parton model introduced in Chapter II, the elementary photon-parton scattering subprocess can be viewed in two different ways. The incident photon can either scatter off a parton or split into a lepton pair. Thus we have two contributions to the process at the amplitude level. According to the first scenario, known as the Compton contribution, a heavy time-like photon, $\gamma^{*}\left(q^{\prime}\right)$ with $q^{\prime 2}>0$, is produced and decays eventually into a pair of leptons, as shown in Fig.

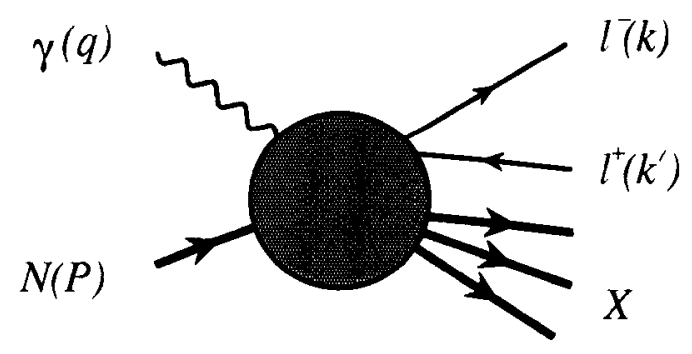

FIG. 20: Inclusive photoproduction of lepton pairs. 


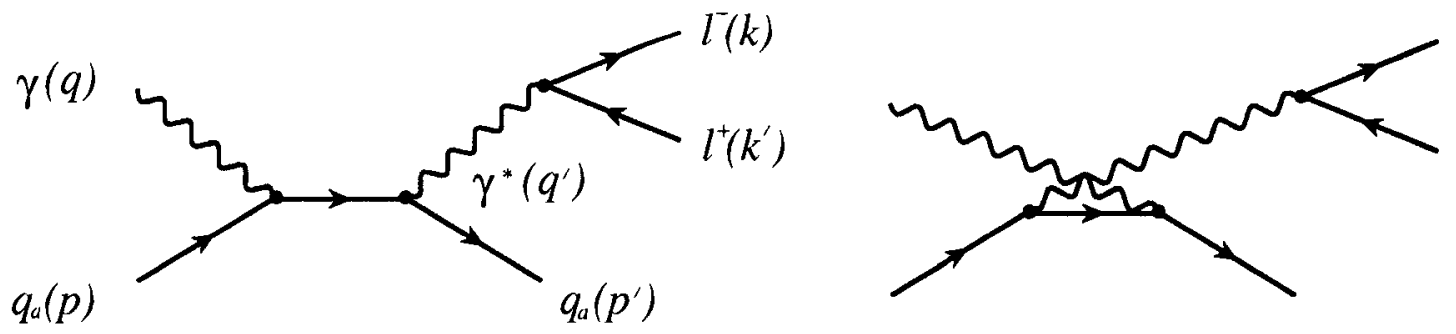

FIG. 21: Compton contribution to the inclusive photoproduction of lepton pairs in the parton model.
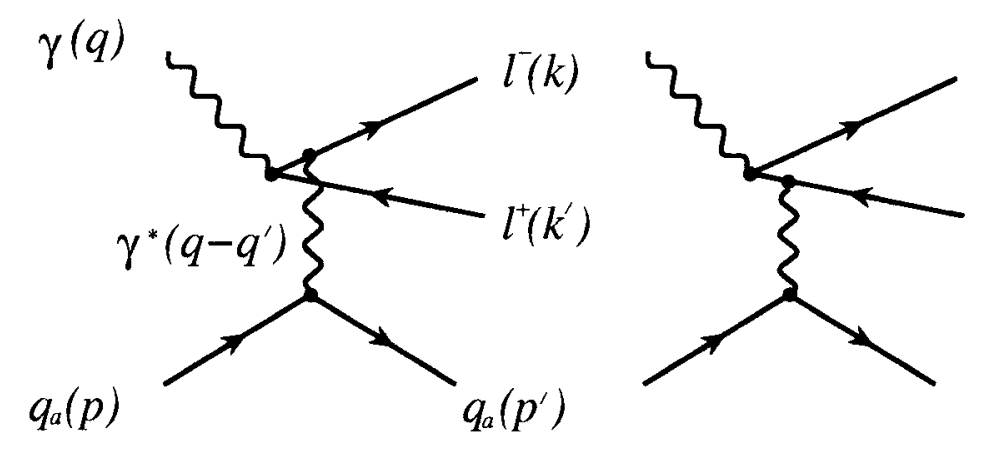

FIG. 22: Bethe-Heitler contribution to the inclusive photoproduction of lepton pairs in the parton model.

21. We discuss this particular subprocess (see also [102]) in Section V.2. The second scenario is illustrated illustrated in Fig. 22 and corresponds to the Bethe-Heitler mechanism. It will be studied in Section V.3. The real and virtual photons can be interchanged, and hence both the Compton and Bethe-Heitler contributions consist of two Feynman diagrams.

\section{V.2 COMPTON SUBPROCESS}

The invariant matrix element for the Compton part comes from two Feynman diagrams shown in Fig. 21. Neglecting the parton masses by taking $p^{2}=p^{2}=0$, we have

$$
i \mathrm{~T}_{C}=\bar{u}(k)\left(i|e| \gamma^{\lambda}\right) v\left(k^{\prime}\right)\left(\frac{-i g_{\mu \lambda}}{q^{2}}\right) \bar{u}\left(p^{\prime}\right)\left(-i Q_{a}|e| \gamma^{\mu}\right) \frac{i(\not p+\not q)}{(p+q)^{2}}
$$




$$
\begin{aligned}
& \times\left(-i Q_{a}|e| \gamma^{\nu}\right) \epsilon_{\nu}(q) u(p) \\
& +\bar{u}(k)\left(i|e| \gamma^{\lambda}\right) v\left(k^{\prime}\right)\left(\frac{-i g_{\mu \lambda}}{q^{2}}\right) \bar{u}\left(p^{\prime}\right)\left(-i Q_{a}|e| \gamma^{\nu}\right) \epsilon_{\nu}(q) \frac{i\left(\not p-\not q^{\prime}\right)}{\left(p-q^{\prime}\right)^{2}} \\
& \times\left(-i Q_{a}|e| \gamma^{\mu}\right) u(p)
\end{aligned}
$$

where $\epsilon_{\nu}(q)$ with $q^{2}=0$ denotes the polarization vector of the initial real photon. By simplifying the numerator of each propagator in Eq. (149), we obtain for the T-matrix

$$
\begin{aligned}
\mathrm{T}_{C}= & -\frac{|e|^{3} Q_{a}^{2}}{q^{2}} \bar{u}(k) \gamma_{\mu} v\left(k^{\prime}\right) \epsilon_{\nu}(q) \bar{u}\left(p^{\prime}\right)\left[\frac{\gamma^{\mu} d \gamma^{\nu}+2 \gamma^{\mu} p^{\nu}}{2(p \cdot q)}\right. \\
& \left.+\frac{-\gamma^{\nu} q^{\prime} \gamma^{\mu}+2 \gamma^{\nu} p^{\mu}}{-2\left(p^{\prime} \cdot q\right)}\right] u(p)
\end{aligned}
$$

We will assume that both the nucleon target and the photon beam are unpolarized. Then the square of Eq. (150) has to be averaged over the initial parton and photon polarizations, and further summed over the final parton and lepton polarizations. It can be written in a factorized form in the following way

$$
\overline{\left|\mathrm{T}_{C}\right|^{2}}=\frac{(4 \pi \alpha)^{3} Q_{a}^{4}}{2 q^{\prime 4}} L_{\mu \rho}^{C} H_{C}^{\mu \rho} .
$$

The lepton tensor is

$$
L_{\mu \rho}^{C}=4\left[k_{\mu} k_{\rho}^{\prime}+k_{\rho} k_{\mu}^{\prime}-g_{\mu \rho}\left[\left(k \cdot k^{\prime}\right)+m^{2}\right]\right]
$$

where $m$ denotes the lepton mass. The hadron tensor, on the other hand, is more complicated. In the Feynman gauge, it reads

$$
\begin{aligned}
H_{C}^{\mu \rho}= & 2\left[\frac{A_{C}}{(p \cdot q)^{2}}+\frac{B_{C}}{\left(p^{\prime} \cdot q\right)^{2}}+\frac{C_{C}}{(p \cdot q)\left(p^{\prime} \cdot q\right)}\right] \\
A_{C}= & (p \cdot q)\left[p^{\prime \mu} q^{\rho}+p^{\prime \rho} q^{\mu}-g^{\mu \rho}\left(p^{\prime} \cdot q\right)\right] \\
B_{C}= & \left(p \cdot q^{\prime}\right)\left[p^{\prime \mu} p^{\rho}+p^{\rho} p^{\mu}\right]+\left(p^{\prime} \cdot q^{\prime}\right)\left[p^{\mu} q^{\prime \rho}+p^{\rho} q^{\prime \mu}-2 p^{\mu} p^{\rho}\right] \\
& +\left(p \cdot p^{\prime}\right)\left[2 p^{\mu} p^{\rho}-p^{\mu} q^{\prime \rho}-p^{\rho} q^{\prime \mu}\right]+\frac{q^{\prime 2}}{2}\left[-p^{\mu} p^{\rho}-p^{\prime \rho} p^{\mu}+g^{\mu \rho}\left(p \cdot p^{\prime}\right)\right] \\
& -g^{\mu \rho}\left(p \cdot q^{\prime}\right)\left(p^{\prime} \cdot q^{\prime}\right), \\
C_{C}= & (p \cdot q)\left[p^{\prime \mu} q^{\rho}+p^{\prime \rho} q^{\prime \mu}-p^{\prime \mu} p^{\rho}-p^{\prime \rho} p^{\mu}\right]+\left(p^{\prime} \cdot q\right)\left[2 p^{\mu} p^{\rho}-p^{\mu} q^{\prime \rho}-p^{\rho} q^{\prime \mu}\right] \\
& +\left(p \cdot p^{\prime}\right)\left[-p^{\mu} q^{\rho}-p^{\rho} q^{\mu}-p^{\mu} q^{\prime \rho}-p^{\rho} q^{\prime \mu}\right] \\
& +g^{\mu \rho}\left[2\left(p \cdot p^{\prime}\right)\left(p \cdot q^{\prime}\right)+\left(q \cdot q^{\prime}\right)\left(p \cdot p^{\prime}\right)+\left(p \cdot q^{\prime}\right)\left(p^{\prime} \cdot q\right)-(p \cdot q)\left(p^{\prime} \cdot q^{\prime}\right)\right]
\end{aligned}
$$


At this point, it is convenient to introduce the Mandelstam variables for the scattering subprocess at the parton level, namely,

$$
\begin{aligned}
& \hat{s}=(p+q)^{2}=\left(p^{\prime}+q^{\prime}\right)^{2}, \\
& \hat{t}=\left(p^{\prime}-p\right)^{2}=\left(q^{\prime}-q\right)^{2}, \\
& \hat{u}=\left(q^{\prime}-p\right)^{2}=\left(p^{\prime}-q\right)^{2} .
\end{aligned}
$$

The four-momentum conservation implies that $\hat{s}+\hat{t}+\hat{u}=q^{2}$. With the help of Eq. (154) one can immediately verify the gauge invariance of the hadron tensor,

$$
\begin{aligned}
q_{\mu}^{\prime} q_{\rho}^{\prime} H_{C}^{\mu \rho}= & 2\left[\frac{\hat{s} q^{\prime 2}-q^{\prime 4}-\hat{s} \hat{t}+q^{2} \hat{t}+q^{\prime 2} \hat{u}}{\hat{s}}+\frac{\left(q^{2}-\hat{u}\right)\left(\hat{u} \hat{t}+q^{2} \hat{t}\right)-q^{\prime 4} \hat{t}}{\hat{u}^{2}}\right. \\
& \left.-\frac{\hat{s} \hat{u}\left(\hat{s}-q^{\prime 2}\right)+\hat{u}^{2}\left(q^{2}-\hat{u}\right)-\hat{u} \hat{t}\left(q^{\prime 2}-\hat{u}\right)}{\hat{s} \hat{u}}\right] \\
= & 2[-\hat{t}-\hat{t}+2 \hat{t}] \\
= & 0 .
\end{aligned}
$$

In addition, by setting $q^{2}=0$ in Eq. (153), in other words, the outgoing photon now becomes real, and also replacing the lepton tensor with the metric tensor $-g_{\mu \rho}$, we easily recover the high-energy limit of the usual Compton scattering process,

$$
\overline{\left|\mathrm{T}_{C}\right|^{2}} \sim-\left[\frac{\hat{u}}{\hat{s}}+\frac{\hat{s}}{\hat{u}}\right] \text {. }
$$

To calculate the unpolarized scattering cross section, we should integrate Eq. (151) over the Lorentz-invariant phase space defined as

$$
d \Pi=(2 \pi)^{4} \delta^{(4)}\left(p+q-p^{\prime}-k-k^{\prime}\right)\left[\frac{d^{3} p^{\prime}}{(2 \pi)^{3} 2 E_{p^{\prime}}}\right]\left[\frac{d^{3} k}{(2 \pi)^{3} 2 \omega}\right]\left[\frac{d^{3} k^{\prime}}{(2 \pi)^{3} 2 \omega^{\prime}}\right]
$$

in a specific frame of reference, and divide the result of integration by the flux factor. The latter is written in the invariant form as $2 \hat{s}$. Since the brute-force contraction of tensors $L_{\mu \rho}^{C}$ and $H_{C}^{\mu \rho}$ is somewhat tedious, we use a little trick instead. One notices that $L_{\mu \rho}^{C}$ depends only on the momenta of the final leptons. For that reason, we can first integrate the lepton tensor over $\vec{k}$ and $\vec{k}^{\prime}$, then contract it with $H_{C}^{\mu \rho}$, and finally perform integration over the remaining momentum $\vec{p}^{\prime}$. Switching to the symmetric momentum variables (i.e. the lepton pair four-momentum, $q^{\prime}=k+k^{\prime}$, and the relative four-momentum, $\left.\kappa=\left(k-k^{\prime}\right) / 2\right)$ and, with the help of the formula

$$
\frac{d^{3} k}{2 \omega}=\int d^{4} k \delta^{+}\left(k^{2}-m^{2}\right)
$$


where $\delta^{+}\left(k^{2}-m^{2}\right) \equiv \delta\left(k^{2}-m^{2}\right) \theta\left(k_{0}\right)$, writing the three-dimensional Lorentzinvariant volume in the momentum space as the four-dimensional integral, we find

$$
\begin{aligned}
\int \frac{d^{3} k}{2 \omega} \int \frac{d^{3} k^{\prime}}{2 \omega^{\prime}} \delta^{(4)}\left(p+q-p^{\prime}-k-k^{\prime}\right) L_{\mu \rho}^{C}= \\
\int d^{4} q^{\prime} \delta^{(4)}\left(p+q-p^{\prime}-q^{\prime}\right)\left\{\int d^{4} \kappa \delta^{+}\left[\left(q^{\prime} / 2+\kappa\right)^{2}-m^{2}\right]\right. \\
\left.\times \delta^{+}\left[\left(q^{\prime} / 2-\kappa\right)^{2}-m^{2}\right] L_{\mu \rho}^{C}\right\} .
\end{aligned}
$$

The lepton tensor is now equal to

$$
L_{\mu \rho}^{C}=4\left[\frac{1}{2} q_{\mu}^{\prime} q_{\rho}^{\prime}-2 \kappa_{\mu} \kappa_{\rho}-g_{\mu \rho} \frac{q^{\prime 2}}{2}\right] .
$$

The integral over the relative momentum on the right-hand-side of Eq. (159) has a tensor structure, and it can be constructed out of the tensors $g_{\mu \rho}$ and $q_{\mu}^{\prime} q_{\rho}^{\prime}$ and scalar functions of the invariant mass of the lepton pair $q^{\prime 2}$. Moreover, the Ward identity implies that this integral must assume the form

$$
\int d^{4} \kappa \delta^{+}\left[\left(q^{\prime} / 2+\kappa\right)^{2}-m^{2}\right] \delta^{+}\left[\left(q^{\prime} / 2-\kappa\right)^{2}-m^{2}\right] L_{\mu \rho}^{C}=\Phi\left(q^{\prime 2}\right)\left[q^{\prime 2} g_{\mu \rho}-q_{\mu}^{\prime} q_{\rho}^{\prime}\right]
$$

where the scalar function $\Phi\left(q^{\prime 2}\right)$ is regular (i.e. it has no pole) at $q^{2}=0$. Next we calculate $\Phi\left(q^{2}\right)$. It is a function of a Lorentz scalar and thus frame independent. As a reference frame, it is convenient to choose the center-of-mass frame of the lepton pair. In this particular frame, the symmetric momenta are simply given by $q^{\prime}=\left(\sqrt{q^{2}}, \overrightarrow{0}\right)$ and $\kappa=(0, \vec{\kappa})$. After contracting both sides of Eq. (161) with $g^{\mu \rho}$, then using

$$
\int d x \int d y \delta(x) \delta(y)=2 \int d x \int d y \delta(x+y) \delta(x-y)
$$

and finally integrating over $\kappa$ (the angular integration simply gives $4 \pi$ ), we get for the scalar function

$$
\begin{aligned}
\Phi\left(q^{2}\right)= & -\frac{4}{3 q^{2}} \int d^{4} \kappa \delta^{+}\left[\left(q^{\prime} / 2+\kappa\right)^{2}-m^{2}\right] \delta^{+}\left[\left(q^{\prime} / 2-\kappa\right)^{2}-m^{2}\right]\left[3 q^{2} / 2+2 \kappa^{2}\right] \\
= & -\frac{16 \pi}{3 q^{\prime 2}} \int d\left(|\vec{\kappa}|^{2}\right) \int d \kappa_{0} \delta^{+}\left[2 \sqrt{q^{\prime 2}} \kappa_{0}\right] \delta^{+}\left[q^{2} / 2+2 \kappa_{0}^{2}-2|\vec{\kappa}|^{2}-2 m^{2}\right] \\
& \times|\vec{\kappa}|\left[3 q^{\prime 2} / 2+2 \kappa_{0}^{2}-2|\vec{\kappa}|^{2}\right] \\
= & -\frac{2 \pi}{3} \frac{q^{2}+2 m^{2}}{q^{\prime 2}} \sqrt{1-\frac{4 m^{2}}{q^{2}}} .
\end{aligned}
$$


Note that the scalar function $\Phi\left(q^{2}\right)<0$. For the sake of completeness, it is worth showing that generalizing the calculation of $\Phi\left(q^{2}\right)<0$ to any reference frame indeed yields the same result. For instance, integration over $\kappa_{0}$ gives

$$
\begin{aligned}
\Phi\left(q^{\prime 2}\right) \sim & \int d(\cos \vartheta) \int d\left(|\vec{\kappa}|^{2}\right)|\vec{\kappa}| \delta^{+}\left[q^{\prime 2} / 2-2|\vec{\kappa}|^{2}\left(1-\left|\vec{q}^{\prime}\right|^{2} \cos ^{2} \vartheta / q_{0}^{\prime 2}\right)-2 m^{2}\right] \\
& \times\left[3 q^{\prime 2} / 2-2|\vec{\kappa}|^{2}\left(1-\left|\vec{q}^{\prime}\right|^{2} \cos ^{2} \vartheta / q_{0}^{\prime 2}\right)\right],
\end{aligned}
$$

where $\vartheta$ is the angle between vectors $\vec{\kappa}$ and $\vec{q}^{\prime}$ in the lepton scattering plane. Furthermore, integrating over $|\vec{\kappa}|^{2}$ together with the substitution $z=\left|\vec{q}^{\prime}\right| \cos \vartheta / q_{0}^{\prime}$ produces the integral of the type

$$
\int_{-\left|\vec{q}^{\prime}\right| / q_{0}^{\prime}}^{\left|\vec{q}^{\prime}\right| / q_{0}^{\prime}} \frac{d z}{\left(1-z^{2}\right)^{3 / 2}}
$$

which leads to the same result for $\Phi\left(q^{2}\right)$ given by Eq. (163).

Thus the double integral (159) reduces now to

$$
\begin{aligned}
\int \frac{d^{3} k}{2 \omega} \int \frac{d^{3} k^{\prime}}{2 \omega^{\prime}} \delta^{(4)}\left(p+q-p^{\prime}-k-k^{\prime}\right) L_{\mu \rho}^{C}= & \int d^{4} q^{\prime} \delta^{(4)}\left(p+q-p^{\prime}-q^{\prime}\right) \\
& \times \Phi\left(q^{\prime 2}\right)\left[q^{\prime 2} g_{\mu \rho}-q_{\mu}^{\prime} q_{\rho}^{\prime}\right] .
\end{aligned}
$$

Contracting the tensor in the integrand with $H_{C}^{\mu \rho}$ can be easily carried out. In terms of the subprocess Mandelstam variables, we obtain

$$
\left[q^{\prime 2} g_{\mu \rho}-q_{\mu}^{\prime} q_{\rho}^{\prime}\right] H_{C}^{\mu \rho}=4 q^{\prime 2}\left[\frac{\hat{u}}{\hat{s}}+\frac{\hat{s}}{\hat{u}}+\frac{2 q^{\prime 2} \hat{t}}{\hat{s} \hat{u}}\right]
$$

Note that the second term on the left-hand side of Eq. (167) gives no contribution due to the condition (155). After combining the result of contraction with Eqs. (151) and (157) and using Eq. (158), this time for the momentum $p^{\prime}$, the unpolarized cross section for the Compton subprocess reads

$$
\begin{aligned}
\sigma_{C}= & \int d^{4} q^{\prime} \int d^{4} p^{\prime} \delta^{(4)}\left(p+q-p^{\prime}-q^{\prime}\right) \delta^{+}\left(p^{2}\right) \frac{1}{2 \hat{s}} \frac{1}{(2 \pi)^{5}} \frac{(4 \pi \alpha)^{3} Q_{a}^{4}}{2 q^{\prime 4}} \\
& \times \Phi\left(q^{2}\right) 4 q^{2}\left[\frac{\hat{u}}{\hat{s}}+\frac{\hat{s}}{\hat{u}}+\frac{2 q^{\prime 2} \hat{t}}{\hat{s} \hat{u}}\right] .
\end{aligned}
$$

The delta function $\delta^{(4)}\left(p+q-p^{\prime}-q^{\prime}\right)$ can be trivially integrated over $p^{\prime}$. The final integral over $q^{\prime}$ is performed in the photon-parton center-of-mass frame. Since we want the cross section $\sigma_{C}$ to be differential in the invariant mass of the lepton pair, an extra substitution

$$
\int d^{4} q^{\prime}=\int d^{4} q^{\prime} \int d M_{p a i r}^{2} \delta\left(q^{2}-M_{p a i r}^{2}\right)
$$


is being made. Then

$$
\begin{aligned}
\sigma_{C}= & \pi \int d M_{\text {pair }}^{2} \int d\left(\cos \theta_{c m}\right) \int d\left(\left|\vec{q}^{\prime}\right|^{2}\right) \int d q_{0}^{\prime} \delta^{+}\left(M_{\text {pair }}^{2}+\hat{s}-2 \sqrt{\hat{s}} q_{0}^{\prime}\right) \\
& \times \delta\left(q_{0}^{\prime 2}-\left|\vec{q}^{\prime}\right|^{2}-M_{\text {pair }}^{2}\right)\left|\vec{q}^{\prime}\right| \frac{1}{\hat{s}} \frac{1}{(2 \pi)^{5}} \frac{(4 \pi \alpha)^{3} Q_{a}^{4}}{M_{p a i r}^{2}} \\
& \times \Phi\left(M_{\text {pair }}^{2}\right)\left[\frac{\hat{u}}{\hat{s}}+\frac{\hat{s}}{\hat{u}}+\frac{2 M_{\text {pair }}^{2} \hat{t}}{\hat{s} \hat{u}}\right],
\end{aligned}
$$

where $\theta_{c m}$ is the angle between the directions of the incoming real and outgoing virtual photons in the photon-parton center-of-mass frame. The center-of-mass energy $E_{c m}$ is equally distributed between the photon and the parton and hence the invariant $\hat{s}=E_{c m}^{2}$. In the differential form, after integrations over $q_{0}^{\prime}$ and $\left|\vec{q}^{\prime}\right|^{2}$, we have

$$
\begin{aligned}
\frac{d^{2} \sigma_{C}}{d M_{\text {pair }}^{2} d\left(\cos \theta_{c m}\right)}= & \pi\left(\frac{\hat{s}-M_{\text {pair }}^{2}}{4 \hat{s}}\right) \frac{1}{\hat{s}} \frac{1}{(2 \pi)^{5}} \frac{(4 \pi \alpha)^{3} Q_{a}^{4}}{M_{\text {pair }}^{2}} \\
& \times \Phi\left(M_{\text {pair }}^{2}\right)\left[\frac{\hat{u}}{\hat{s}}+\frac{\hat{s}}{\hat{u}}+\frac{2 M_{\text {pair }}^{2} \hat{t}}{\hat{s} \hat{u}}\right]
\end{aligned}
$$

Finally, replacing $\cos \theta_{c m}$ by the invariant $\hat{t}$ through the relation $\hat{t}=M_{\text {pair }}^{2}-$ $\sqrt{\hat{s}}\left(q_{0}^{\prime}-\sqrt{q_{0}^{\prime 2}-M_{\text {pair }}^{2}} \cos \theta_{c m}\right)$, where $q_{0}^{\prime}=\left(\hat{s}+M_{\text {pair }}^{2}\right) / 2 \sqrt{\hat{s}}$, we arrive at the partonic cross section

$$
\begin{aligned}
\frac{d^{2} \sigma_{C}}{d M_{\text {pair }}^{2} d \hat{t}}= & -\left(\frac{2 \alpha^{3} Q_{a}^{4}}{3}\right)\left(1+\frac{2 m^{2}}{M_{\text {pair }}^{2}}\right) \sqrt{1-\frac{4 m^{2}}{M_{p a i r}^{2}}} \frac{1}{\hat{s}^{2} M_{p a i r}^{2}} \\
& \times\left[\frac{\hat{s}^{2}+\hat{u}^{2}+2 M_{\text {pair }}^{2} \hat{t}}{\hat{s} \hat{u}}\right] .
\end{aligned}
$$

We calculate now the cross section for photon-nucleon inelastic scattering with the help of the parton model master formula, see Section II.3. The process cross section is obtained by summing Eq. (172) over all types $a$ of charged partons and all possible longitudinal momentum fractions $x$, where the single summands must be weighted with the proper PDFs $f_{a / N}(x)$. Namely,

$$
\begin{array}{r}
\sigma\left[\gamma(q) N(P) \rightarrow l^{-}(k) l^{+}\left(k^{\prime}\right) X\right]= \\
\sum_{a} \int_{0}^{1} d x f_{a / N}(x) \sigma\left[\gamma(q) q_{a}(x P) \rightarrow l^{-}(k) l^{+}\left(k^{\prime}\right) q_{a}\left(p^{\prime}\right)\right] .
\end{array}
$$

In addition, the subprocess Mandelstam variables are expressed in terms of the process variables. The invariant $\hat{t}$ is identical to $t \equiv\left(q^{\prime}-q\right)^{2}$. Neglecting the nucleon mass, the invariant $\hat{s}$ is simply given by $\hat{s}=x s$, where $s \equiv(P+q)^{2}$. Accordingly, the 
Mandelstam invariant $\hat{u}=M_{\text {pair }}^{2}-t-x s$. Recall that by virtue of the vanishing mass of the scattered parton, $p^{2}=\left(x P+q-q^{\prime}\right)^{2}=0$, the longitudinal momentum fraction coincides with the Bjorken scaling variable,

$$
x=-\frac{t}{2\left[P \cdot\left(q-q^{\prime}\right)\right]} \equiv x_{B}
$$

Substituting Eq. (172) into Eq. (173) ultimately yields the Compton differential cross section for the inclusive photoproduction of lepton pairs in the parton model

$$
\begin{aligned}
& \frac{d^{3} \sigma_{C}\left(\gamma N \rightarrow l^{-} l^{+} X\right)}{d M_{\text {pair }}^{2} d t d x_{B}}=\sum_{a} \int_{0}^{1} d x \delta\left(x-x_{B}\right) f_{a / N}(x)\left\{\frac{2 \alpha^{3} Q_{a}^{4}}{3}\left(1+\frac{2 m^{2}}{M_{p a i r}^{2}}\right)\right. \\
& \times \sqrt{1-\frac{4 m^{2}}{M_{p a i r}^{2}}} \frac{1}{(x s)^{2} M_{p a i r}^{2}} \\
& \left.\times\left[\frac{(x s)^{2}+\left(M_{\text {pair }}^{2}-t-x s\right)^{2}+2 M_{\text {pair }}^{2} t}{x s\left(x s+t-M_{\text {pair }}^{2}\right)}\right]\right\} \\
& =\frac{2 \alpha^{3}}{3}\left(1+\frac{2 m^{2}}{M_{\text {pair }}^{2}}\right) \sqrt{1-\frac{4 m^{2}}{M_{\text {pair }}^{2}}} \frac{1}{\left(x_{B} s\right)^{2} M_{\text {pair }}^{2}} \\
& \times\left[\frac{\left(x_{B} s\right)^{2}+\left(M_{p a i r}^{2}-t-x_{B} s\right)^{2}+2 M_{p a i r}^{2} t}{x_{B} s\left(x_{B} s+t-M_{p a i r}^{2}\right)}\right] \\
& \times \sum_{a} Q_{a}^{4} f_{a / N}\left(x_{B}\right) \text {. }
\end{aligned}
$$

\section{V.3 BETHE-HEITLER SUBPROCESS}

The Bethe-Heitler amplitude is calculated from the Feynman diagrams shown in Fig. 22. Adopting the notation of the preceding subsection, we write

$$
\begin{aligned}
i \mathrm{~T}_{B H}= & \bar{u}\left(p^{\prime}\right)\left(-i Q_{a}|e| \gamma^{\lambda}\right) u(p)\left(\frac{-i g_{\mu \lambda}}{\left(q-q^{\prime}\right)^{2}}\right) \bar{u}(k)\left(i|e| \gamma^{\mu}\right) \frac{i\left(\not-\not k^{\prime}+m\right)}{\left(q-k^{\prime}\right)^{2}-m^{2}} \\
& \times\left(i|e| \gamma^{\nu}\right) \epsilon_{\nu}(q) v\left(k^{\prime}\right) \\
& +\bar{u}\left(p^{\prime}\right)\left(-i Q_{a}|e| \gamma^{\lambda}\right) u(p)\left(\frac{-i g_{\mu \lambda}}{\left(q-q^{\prime}\right)^{2}}\right) \bar{u}(k)\left(i|e| \gamma^{\nu}\right) \epsilon_{\nu}(q) \frac{i(\not k-\not q+m)}{(k-q)^{2}-m^{2}} \\
& \times\left(i|e| \gamma^{\mu}\right) v\left(k^{\prime}\right)
\end{aligned}
$$

or

$$
\begin{aligned}
\mathrm{T}_{B H}= & \frac{|e|^{3} Q_{a}}{t} \bar{u}\left(p^{\prime}\right) \gamma_{\mu} u(p) \epsilon_{\nu}(q) \bar{u}(k)\left[\frac{\gamma^{\mu} \not \gamma^{\nu}-2 \gamma^{\mu} k^{\prime \nu}}{-2\left(k^{\prime} \cdot q\right)}\right. \\
& \left.+\frac{-\gamma^{\nu} \not \gamma^{\mu}+2 k^{\nu} \gamma^{\mu}}{-2(k \cdot q)}\right] v\left(k^{\prime}\right)
\end{aligned}
$$


Again, after averaging $\left|\mathrm{T}_{B H}\right|^{2}$ over the polarization states of the initial particles and summing over the polarization states of the final parton, we have for the spin-averaged square of the amplitude

$$
\overline{\left|\mathrm{T}_{B H}\right|^{2}}=\frac{(4 \pi \alpha)^{3} Q_{a}^{2}}{2 t^{2}} L_{\mu \rho}^{B H} H_{B H}^{\mu \rho}
$$

A straightforward calculation of traces gives

$$
\begin{aligned}
L_{B H}^{\mu \rho}= & 4\left[\frac{A_{B H}}{(k \cdot q)^{2}}+\frac{B_{B H}}{\left(k^{\prime} \cdot q\right)^{2}}+\frac{C_{B H}}{(k \cdot q)\left(k^{\prime} \cdot q\right)}\right] \\
A_{B H}= & (k \cdot q)\left[k^{\prime \mu} q^{\rho}+k^{\prime \rho} q^{\mu}-g^{\mu \rho}\left(k^{\prime} \cdot q\right)\right]-m^{2}\left[k^{\mu} k^{\prime \rho}+k^{\rho} k^{\prime \mu}-g^{\mu \rho}\left(k \cdot k^{\prime}\right)\right] \\
& +m^{2}\left[k^{\prime \mu} q^{\rho}+k^{\prime \rho} q^{\mu}-g^{\mu \rho}\left(k^{\prime} \cdot q\right)\right]-m^{2}(k \cdot q) g^{\mu \rho}+m^{4} g^{\mu \rho} \\
B_{B H}= & \left(k^{\prime} \cdot q\right)\left[k^{\mu} q^{\rho}+k^{\rho} q^{\mu}-g^{\mu \rho}(k \cdot q)\right]-m^{2}\left[k^{\mu} k^{\prime \rho}+k^{\rho} k^{\prime \mu}-g^{\mu \rho}\left(k \cdot k^{\prime}\right)\right] \\
& +m^{2}\left[k^{\mu} q^{\rho}+k^{\rho} q^{\mu}-g^{\mu \rho}(k \cdot q)\right]-m^{2}\left(k^{\prime} \cdot q\right) g^{\mu \rho}+m^{4} g^{\mu \rho} \\
C_{B H}= & 2\left(k \cdot k^{\prime}\right)\left[k^{\mu} k^{\prime \rho}+k^{\rho} k^{\prime \mu}-g^{\mu \rho}\left(k \cdot k^{\prime}\right)\right]-(k \cdot q)\left[k^{\mu} k^{\prime \rho}+k^{\rho} k^{\prime \mu}-2 k^{\prime \mu} k^{\prime \rho}\right] \\
& -\left(k^{\prime} \cdot q\right)\left[k^{\mu} k^{\prime \rho}+k^{\rho} k^{\prime \mu}-2 k^{\mu} k^{\rho}\right]-\left(k \cdot k^{\prime}\right)\left[k^{\mu} q^{\rho}+k^{\rho} q^{\mu}+k^{\prime \mu} q^{\rho}+k^{\prime \rho} q^{\mu}\right] \\
& +2 g^{\mu \rho}\left(k \cdot k^{\prime}\right)\left[(k \cdot q)+\left(k^{\prime} \cdot q\right)-m^{2}\right]-2 m^{2} q^{\mu} q^{\rho}
\end{aligned}
$$

for the lepton tensor and

$$
H_{\mu \rho}^{B H}=2\left[p_{\mu} p_{\rho}^{\prime}+p_{\rho} p_{\mu}^{\prime}-g_{\mu \rho}\left(p \cdot p^{\prime}\right)\right]
$$

for the hadron tensor.

Recall now that the expression (178) has to be integrated over the final-state momenta constrained by a four-momentum delta function, see Eq. (157). However, before contracting tensors, we perform integration of $L_{B H}^{\mu \rho}$ over both lepton threemomenta. One defines this particular integral as

$$
\mathcal{L}_{B H}^{\mu \rho} \equiv \int \frac{d^{3} k}{2 \omega} \int \frac{d^{3} k^{\prime}}{2 \omega^{\prime}} \delta^{(4)}\left(q^{\prime}-k-k^{\prime}\right) L_{B H}^{\mu \rho},
$$

which, with the help of the formula (158), turns into

$$
\mathcal{L}_{B H}^{\mu \rho} \equiv \int d^{4} k \int d^{4} k^{\prime} \delta^{(4)}\left(q^{\prime}-k-k^{\prime}\right) \delta^{+}\left(k^{2}-m^{2}\right) \delta^{+}\left(k^{2}-m^{2}\right) L_{B H}^{\mu \rho} .
$$

We evaluate $\mathcal{L}_{B H}^{\mu \rho}$ in the center-of-mass frame of the lepton pair. Unfortunately, due to presence of a large number of terms in the lepton tensor, the calculation requires 
more algebra. Let us demonstrate the method by calculating only the integral of the first term in the tensor (179), namely,

$$
\mathcal{L}_{B H(1)}^{\mu \rho}=\int d^{4} k \int d^{4} k^{\prime} \delta^{(4)}\left(q^{\prime}-k-k^{\prime}\right) \delta^{+}\left(k^{2}-m^{2}\right) \delta^{+}\left(k^{\prime 2}-m^{2}\right)\left[\frac{4 k^{\prime \mu} q^{\rho}}{(k \cdot q)}\right] .
$$

Integration over $k^{\prime}$ leads to

$$
\begin{aligned}
\mathcal{L}_{B H(1)}^{\mu \rho}= & 4 q^{\prime \mu} q^{\rho} \int d^{4} k \delta^{+}\left(k^{2}-m^{2}\right) \delta^{+}\left[\left(q^{\prime}-k\right)^{2}-m^{2}\right]\left[\frac{1}{(k \cdot q)}\right] \\
& -4 q^{\rho} \int d^{4} k \delta^{+}\left(k^{2}-m^{2}\right) \delta^{+}\left[\left(q^{\prime}-k\right)^{2}-m^{2}\right]\left[\frac{k^{\mu}}{(k \cdot q)}\right]
\end{aligned}
$$

For simplicity, we choose the incident photon momentum along the $z$-axis so that $q=\left(q_{0}, 0,0, q_{0}\right)$. The four-momentum of the outgoing lepton is $k=\left(k_{0}, \vec{k}_{\perp}, k_{z}\right)$. Accordingly, the calculation of the first integral in Eq. (184) (we will call it the function $I_{0}$ for later convenience) goes as follows

$$
\begin{aligned}
I_{0}= & \pi \int d k_{z} \int d\left(\left|\vec{k}_{\perp}\right|^{2}\right) \int d k_{0} \delta^{+}\left(k_{0}^{2}-\left|\vec{k}_{\perp}\right|^{2}-k_{z}^{2}-m^{2}\right) \delta^{+}\left(q^{\prime 2}-2 \sqrt{q^{2}} k_{0}\right) \\
& \times \frac{1}{q_{0}\left(k_{0}-k_{z}\right)} \\
= & -\frac{\pi}{2 \sqrt{q^{\prime 2}} q_{0}} \int_{-\sqrt{q^{\prime 2} / 4-m^{2}}}^{\sqrt{q^{\prime 2} / 4-m^{2}}} \frac{d k_{z}}{k_{z}-\sqrt{q^{\prime 2}} / 2} \\
= & \frac{\pi}{2\left(q \cdot q^{\prime}\right)} \ln \left[\frac{1+\sqrt{1-4 m^{2} / q^{\prime 2}}}{1-\sqrt{1-4 m^{2} / q^{\prime 2}}}\right] .
\end{aligned}
$$

Note that we have expressed the initial photon energy in the invariant form, $q_{0}=$ $\left(q \cdot q^{\prime}\right) / \sqrt{q^{\prime 2}}$. The integral over $k$ in the second term of Eq. (184) has a Lorentz vector-like structure and can be, in general, written as

$$
\int d^{4} k \delta^{+}\left(k^{2}-m^{2}\right) \delta^{+}\left[\left(q^{\prime}-k\right)^{2}-m^{2}\right]\left[\frac{k^{\mu}}{(k \cdot q)}\right]=I_{1} q^{\mu}+I_{2} q^{\prime \mu}
$$

The scalar functions $I_{1}$ and $I_{2}$ are determined by solving the system of two equations,

$$
\begin{aligned}
\int d^{4} k \delta^{+}\left(k^{2}-m^{2}\right) \delta^{+}\left[\left(q^{\prime}-k\right)^{2}-m^{2}\right] & =\left(q \cdot q^{\prime}\right) I_{2} \\
\int d^{4} k \delta^{+}\left(k^{2}-m^{2}\right) \delta^{+}\left[\left(q^{\prime}-k\right)^{2}-m^{2}\right] \frac{\left(k \cdot q^{\prime}\right)}{(k \cdot q)} & =\left[\left(q \cdot q^{\prime}\right) I_{1}+q^{2} I_{2}\right]
\end{aligned}
$$


obtained from contracting both sides of Eq. (186) with $q_{\mu}$ and $q_{\mu}^{\prime}$, respectively. After some algebra we find

$$
\begin{aligned}
I_{2} & =\frac{\pi}{2\left(q \cdot q^{\prime}\right)} \sqrt{1-\frac{4 m^{2}}{q^{2}}} \\
I_{1} & =\frac{q^{\prime 2}}{2\left(q \cdot q^{\prime}\right)}\left[I_{0}-2 I_{2}\right] \\
& =\frac{\pi q^{\prime 2}}{4\left(q \cdot q^{\prime}\right)^{2}}\left\{\ln \left[\frac{1+\sqrt{1-4 m^{2} / q^{\prime 2}}}{1-\sqrt{1-4 m^{2} / q^{\prime 2}}}\right]-2 \sqrt{1-\frac{4 m^{2}}{q^{2}}}\right\}
\end{aligned}
$$

The term (183) now becomes equal to

$$
\mathcal{L}_{B H(1)}^{\mu \rho}=-4 I_{1} q^{\mu} q^{\rho}+4\left(I_{0}-I_{2}\right) q^{\prime \mu} q^{\rho}
$$

We repeat this procedure for all the remaining terms in the lepton tensor (all the integrals with the corresponding scalar functions are presented in Appendix B), and combine them together according to their structure. The final expression for the lepton tensor then reads

$$
\mathcal{L}_{B H}^{\mu \rho}=\frac{2 \pi}{\left(q \cdot q^{\prime}\right)}\left[\mathcal{A}_{B H} q^{\mu} q^{\rho}+\mathcal{B}_{B H} q^{\prime \mu} q^{\prime \rho}+\mathcal{C}_{B H}\left(q^{\mu} q^{\prime \rho}+q^{\prime \mu} q^{\rho}\right)+\mathcal{D}_{B H} g^{\mu \rho}\right]
$$

where the coefficients

$$
\begin{aligned}
\mathcal{A}_{B H}= & {\left[\frac{2 q^{\prime 2}}{\left(q \cdot q^{\prime}\right)}-\frac{2 q^{\prime 4}}{\left(q \cdot q^{\prime}\right)^{2}}+\frac{q^{\prime 6}}{\left(q \cdot q^{\prime}\right)^{3}}-\frac{8 m^{2} q^{\prime 2}}{\left(q \cdot q^{\prime}\right)^{2}}-\frac{4 m^{4} q^{2}}{\left(q \cdot q^{\prime}\right)^{3}}+\frac{6 m^{2} q^{\prime 4}}{\left(q \cdot q^{\prime}\right)^{3}}+\frac{4 m^{2}}{\left(q \cdot q^{\prime}\right)}\right] } \\
& \times \ln \left[\frac{1-\sqrt{1-4 m^{2} / q^{\prime 2}}}{1+\sqrt{1-4 m^{2} / q^{\prime 2}}}\right] \\
& +\left[\frac{4 q^{\prime 2}}{\left(q \cdot q^{\prime}\right)}-\frac{8 q^{\prime 4}}{\left(q \cdot q^{\prime}\right)^{2}}+\frac{4 q^{\prime 6}}{\left(q \cdot q^{\prime}\right)^{3}}+\frac{2 m^{2} q^{\prime 4}}{\left(q \cdot q^{\prime}\right)^{3}}\right] \sqrt{1-\frac{4 m^{2}}{q^{\prime 2}}} \\
\mathcal{B}_{B H}= & \frac{4 m^{2}}{\left(q \cdot q^{\prime}\right)} \ln \left[\frac{1-\sqrt{1-4 m^{2} / q^{\prime 2}}}{1+\sqrt{1-4 m^{2} / q^{\prime 2}}}\right]+\frac{2 q^{2}}{\left(q \cdot q^{\prime}\right)} \sqrt{1-\frac{4 m^{2}}{q^{\prime 2}}} \\
\mathcal{C}_{B H}= & {\left[-2+\frac{2 q^{2}}{\left(q \cdot q^{\prime}\right)}-\frac{q^{\prime 4}}{\left(q \cdot q^{\prime}\right)^{2}}-\frac{6 m^{2} q^{\prime 2}}{\left(q \cdot q^{\prime}\right)^{2}}+\frac{4 m^{2}}{\left(q \cdot q^{\prime}\right)}+\frac{4 m^{4}}{\left(q \cdot q^{\prime}\right)^{2}}\right] } \\
& \times \ln \left[\frac{1-\sqrt{1-4 m^{2} / q^{\prime 2}}}{1+\sqrt{1-4 m^{2} / q^{\prime 2}}}\right] \\
& +\left[-2+\frac{6 q^{\prime 2}}{\left(q \cdot q^{\prime}\right)}-\frac{4 q^{\prime 4}}{\left(q \cdot q^{\prime}\right)^{2}}-\frac{2 m^{2} q^{2}}{\left(q \cdot q^{\prime}\right)^{2}}\right] \sqrt{1-\frac{4 m^{2}}{q^{2}}}
\end{aligned}
$$




$$
\begin{aligned}
\mathcal{D}_{B H}= & {\left[2\left(q \cdot q^{\prime}\right)-2 q^{2}+\frac{q^{\prime 4}}{\left(q \cdot q^{\prime}\right)}+\frac{2 m^{2} q^{\prime 2}}{\left(q \cdot q^{\prime}\right)}-\frac{4 m^{4}}{\left(q \cdot q^{\prime}\right)}\right] \ln \left[\frac{1-\sqrt{1-4 m^{2} / q^{\prime 2}}}{1+\sqrt{1-4 m^{2} / q^{\prime 2}}}\right] } \\
& +\left[2\left(q \cdot q^{\prime}\right)-4 q^{\prime 2}+\frac{2 q^{\prime 4}}{\left(q \cdot q^{\prime}\right)}+\frac{2 m^{2} q^{\prime 2}}{\left(q \cdot q^{\prime}\right)}\right] \sqrt{1-\frac{4 m^{2}}{q^{2}}}
\end{aligned}
$$

are scalar functions of the invariants $m^{2}, q^{2}$ and $\left(q \cdot q^{\prime}\right)=\left(q^{2}-t\right) / 2$.

For the sake of completeness, it is worth noting that the same method can be applied to compute the integral of the lepton tensor, $L_{\mu \rho}^{C}=4\left[k_{\mu} k_{\rho}^{\prime}+k_{\rho} k_{\mu}^{\prime}-g_{\mu \rho} q^{2} / 2\right]$, given by Eq. (152) (note that $\left(k \cdot k^{\prime}\right)+m^{2}=q^{2} / 2$ ), over the final lepton momenta for the Compton subprocess, namely,

$$
\mathcal{L}_{\mu \rho}^{C}=\int d^{4} k \int d^{4} k^{\prime} \delta^{(4)}\left(q^{\prime}-k-k^{\prime}\right) \delta^{+}\left(k^{2}-m^{2}\right) \delta^{+}\left(k^{2}-m^{2}\right) L_{\mu \rho}^{C}
$$

Using $\delta^{(4)}\left(q^{\prime}-k-k^{\prime}\right)$ to integrate over $k^{\prime}$ and then writing each term separately we get

$$
\begin{aligned}
\mathcal{L}_{\mu \rho}^{C}= & 4 q_{\rho}^{\prime} \int d^{4} k \delta^{+}\left(k^{2}-m^{2}\right) \delta^{+}\left[\left(q^{\prime}-k\right)^{2}-m^{2}\right] k_{\mu} \\
& +4 q_{\mu}^{\prime} \int d^{4} k \delta^{+}\left(k^{2}-m^{2}\right) \delta^{+}\left[\left(q^{\prime}-k\right)^{2}-m^{2}\right] k_{\rho} \\
& -8 \int d^{4} k \delta^{+}\left(k^{2}-m^{2}\right) \delta^{+}\left[\left(q^{\prime}-k\right)^{2}-m^{2}\right] k_{\mu} k_{\rho} \\
& -2 g_{\mu \rho} q^{2} \int d^{4} k \delta^{+}\left(k^{2}-m^{2}\right) \delta^{+}\left[\left(q^{\prime}-k\right)^{2}-m^{2}\right] .
\end{aligned}
$$

These integrals can only be constructed out of a four-vector $q^{\prime}$ and a metric tensor, accompanied with the proper scalar functions of $q^{2}$. One can immediately discard the first two integrals. They are both of the form $q_{\mu}^{\prime} q_{\rho}^{\prime}$ and, since Eq. (155) holds, they give vanishing contribution, when contracted with $H_{C}^{\mu \rho}$. Moreover, we write the third integral as

$$
\int d^{4} k \delta^{+}\left(k^{2}-m^{2}\right) \delta^{+}\left[\left(q^{\prime}-k\right)^{2}-m^{2}\right] k_{\mu} k_{\rho}=N_{1} q_{\mu}^{\prime} q_{\rho}^{\prime}+N_{2} g_{\mu \rho}
$$

Contracting both sides with $q^{\mu} q^{\rho}$ and $g^{\mu \rho}$ and evaluating the integral, we find the solutions for the scalar functions

$$
\begin{aligned}
& N_{1}=\frac{\pi}{2 q^{2}} \sqrt{1-\frac{4 m^{2}}{q^{2}}}\left(q^{2}-\frac{m^{2}}{3}\right) \\
& N_{2}=-\frac{\pi}{6} \sqrt{1-\frac{4 m^{2}}{q^{2}}}\left(\frac{q^{2}}{4}-m^{2}\right)
\end{aligned}
$$


Again, due to the gauge invariance of $H_{C}^{\mu \rho}$ only the term $N_{2} g_{\mu \rho}$ survives. Lastly, the final integral in Eq. (193) can be read off from Eqs. (187) and (188),

$$
\int d^{4} k \delta^{+}\left(k^{2}-m^{2}\right) \delta^{+}\left[\left(q^{\prime}-k\right)^{2}-m^{2}\right]=\frac{\pi}{2} \sqrt{1-\frac{4 m^{2}}{q^{2}}} .
$$

By collecting terms, $\mathcal{L}_{\mu \rho}^{C}$ reduces to

$$
\begin{aligned}
\mathcal{L}_{\mu \rho}^{C} & =\left(-8 N_{2}-2 q^{\prime 2}\right) g_{\mu \rho} \\
& =-\frac{2 \pi}{3}\left(q^{2}+2 m^{2}\right) \sqrt{1-\frac{4 m^{2}}{q^{2}}} g_{\mu \rho},
\end{aligned}
$$

which is identical, apart from the vanishing contribution $q_{\mu}^{\prime} q_{\rho}^{\prime}$, to the expression (161).

Next we contract Eq. (190) with the hadron tensor (180) and follow the steps from Eq. (168) to Eq. (172) leading to the Bethe-Heitler differential partonic cross section, expressed in terms of the subprocess Mandelstam invariants

$$
\frac{d^{2} \sigma_{B H}}{d M_{\text {pair }}^{2} d \hat{t}}=\left(\alpha^{3} Q_{a}^{2}\right) \frac{1}{\hat{s}^{2} \hat{t}^{2}\left(M_{\text {pair }}^{2}-\hat{t}\right)}\left[-\mathcal{E}_{B H} \hat{u} \hat{s}+\mathcal{F}_{B H} \hat{t}\right] .
$$

The new coefficients are given by $\mathcal{E}_{B H}=\mathcal{A}_{B H}+\mathcal{B}_{B H}+2 \mathcal{C}_{B H}$ and $\mathcal{F}_{B H}=2 \mathcal{D}_{B H}$ together with the replacement $q^{2} \rightarrow M_{\text {pair }}^{2}$, namely,

$$
\begin{aligned}
\mathcal{E}_{B H}= & {\left[-4+\frac{6 M_{\text {pair }}^{2}}{\left(q \cdot q^{\prime}\right)}-\frac{4 M_{\text {pair }}^{4}}{\left(q \cdot q^{\prime}\right)^{2}}+\frac{M_{\text {pair }}^{6}}{\left(q \cdot q^{\prime}\right)^{3}}-\frac{20 m^{2} M_{\text {pair }}^{2}}{\left(q \cdot q^{\prime}\right)^{2}}-\frac{4 m^{4} M_{\text {pair }}^{2}}{\left(q \cdot q^{\prime}\right)^{3}}+\frac{6 m^{2} M_{\text {pair }}^{4}}{\left(q \cdot q^{\prime}\right)^{3}}\right.} \\
& \left.+\frac{16 m^{2}}{\left(q \cdot q^{\prime}\right)}+\frac{8 m^{4}}{\left(q \cdot q^{\prime}\right)^{2}}\right] \times \ln \left[\frac{1-\sqrt{1-4 m^{2} / M_{\text {pair }}^{2}}}{1+\sqrt{1-4 m^{2} / M_{\text {pair }}^{2}}}\right], \\
\mathcal{F}_{B H}= & {\left[4\left(q \cdot q^{\prime}\right)-4 M_{\text {pair }}^{2}+\frac{2 M_{\text {pair }}^{4}}{\left(q \cdot q^{\prime}\right)}+\frac{4 m^{2} M_{\text {pair }}^{2}}{\left(q \cdot q^{\prime}\right)}-\frac{8 m^{4}}{\left(q \cdot q^{\prime}\right)}\right] } \\
& \times \ln \left[\frac{1-\sqrt{1-4 m^{2} / M_{\text {pair }}^{2}}}{1+\sqrt{1-4 m^{2} / M_{\text {pair }}^{2}}}\right] \\
& +\left[4\left(q \cdot q^{\prime}\right)-8 M_{\text {pair }}^{2}+\frac{4 M_{\text {pair }}^{4}}{\left(q \cdot q^{\prime}\right)}+\frac{4 m^{2} M_{\text {pair }}^{2}}{\left(q \cdot q^{\prime}\right)}\right] \sqrt{1-\frac{4 m^{2}}{M_{\text {pair }}^{2}}} .
\end{aligned}
$$

Finally, with the help of the master formula (173), we obtain the Bethe-Heitler contribution to the inclusive photoproduction of lepton pairs in the parton model

$$
\begin{aligned}
\frac{d^{3} \sigma_{B H}\left(\gamma N \rightarrow l^{-} l^{+} X\right)}{d M_{\text {pair }}^{2} d t d x_{B}}= & \alpha^{3} \frac{1}{\left(x_{B} s\right)^{2} t^{2}\left(M_{\text {pair }}^{2}-t\right)} \\
& \times\left[\mathcal{E}_{B H}\left(x_{B} s+t-M_{\text {pair }}^{2}\right) x_{B} s+\mathcal{F}_{B H} t\right] \\
& \times \sum_{a} Q_{a}^{2} f_{a / N}\left(x_{B}\right) .
\end{aligned}
$$




\section{V.4 INTERFERENCE TERMS}

We turn our attention to the interference between the Compton and Bethe-Heitler subprocesses. Four Feynman diagrams give rise to eight interference terms. They all are calculated in a similar way. For illustration, let us consider the first two terms. Up to a common factor we have

$$
\begin{aligned}
& \overline{\left|\mathrm{T}_{1}\right|^{2}} \sim \frac{1}{\left(k^{\prime} \cdot q\right)} \operatorname{Tr}\left[\not \not^{\prime} \gamma^{\mu}(\not \not p+\not) \gamma^{\nu} \not p \gamma_{\xi}\right] \operatorname{Tr}\left[(\not k+m) \gamma_{\mu}\left(\not k^{\prime}-m\right) \gamma_{\nu}\left(\not \not-\not k^{\prime}+m\right) \gamma^{\xi}\right], \\
& \overline{\left|\mathrm{T}_{2}\right|^{2}} \sim \frac{1}{(k \cdot q)} \operatorname{Tr}\left[\not \not^{\prime} \gamma^{\mu}(\not p+\not q) \gamma^{\nu} \not p \gamma_{\xi}\right] \operatorname{Tr}\left[(\not k+m) \gamma_{\mu}\left(\not \not^{\prime}-m\right) \gamma^{\xi}(\not k-\not h+m) \gamma_{\nu}\right] .
\end{aligned}
$$

After evaluating both second traces in the above expressions, we observe that they differ, apart from the sign, by the interchange $k \leftrightarrow k^{\prime}$. Thus integrating the sum of $\overline{\left|\mathrm{T}_{1}\right|^{2}}$ and $\overline{\left|\mathrm{T}_{2}\right|^{2}}$ over the final lepton four-momenta yields zero,

$$
\int d^{4} k \int d^{4} k^{\prime}\left(\overline{\left|\mathrm{T}_{1}\right|^{2}}+\overline{\left|\mathrm{T}_{2}\right|^{2}}\right)=0 .
$$

By the same token, the remaining six interference terms mutually cancel each other, after being integrated over $k$ and $k^{\prime}$.

In fact, there is a more elegant way (without cumbersome and explicit calculation of traces) to see the cancellation of the interference terms. Note that one needs to compare the following two double integrals

$$
\begin{gathered}
\int d^{4} k \int d^{4} k^{\prime} \delta^{(4)}\left(q^{\prime}-k-k^{\prime}\right) \frac{1}{\left(k^{\prime} \cdot q\right)} \operatorname{Tr}\left[(\not k+m) \gamma_{\mu}\left(\not k^{\prime}-m\right) \gamma_{\nu}\left(\not \not-\not k^{\prime}+m\right) \gamma^{\xi}\right], \\
\int d^{4} k \int d^{4} k^{\prime} \delta^{(4)}\left(q^{\prime}-k-k^{\prime}\right) \frac{1}{(k \cdot q)} \operatorname{Tr}\left[(\not k+m) \gamma_{\mu}\left(\not k^{\prime}-m\right) \gamma^{\xi}(\not k-\not q+m) \gamma_{\nu}\right] .
\end{gathered}
$$

Due to the presence of the delta function, one of the integrals, e.g. the integral over $k^{\prime}$, can be trivially carried out. In addition, using the fact that the trace does not change under the cyclic permutation, Eq. (203) can be rewritten into

$$
\begin{array}{r}
\int d^{4} k \frac{1}{\left[\left(q^{\prime}-k\right) \cdot q\right]} \operatorname{Tr}\left[\gamma_{\mu}\left(\not q^{\prime}-\not k-m\right) \gamma_{\nu}\left(\not d-\not q^{\prime}+\not k+m\right) \gamma^{\xi}(\not k+m)\right], \\
\int d^{4} k \frac{1}{(k \cdot q)} \operatorname{Tr}\left[\gamma_{\mu}\left(\not^{\prime}-\not k-m\right) \gamma^{\xi}(\not k-\not q+m) \gamma_{\nu}(\not k+m)\right] .
\end{array}
$$

Transposing now the trace in the first integrand, and then performing the momentum shift, $\tilde{k}=q^{\prime}-k$, this integral turns into

$$
\int d^{4} \tilde{k} \frac{1}{(\tilde{k} \cdot q)} \operatorname{Tr}\left[\left(\not q^{\prime}-\tilde{k}+m\right)^{\mathrm{T}} \gamma^{\xi^{\mathrm{T}}}(\not-\tilde{k}+m)^{\mathrm{T}} \gamma_{\nu}^{\mathrm{T}}(\tilde{k}-m)^{\mathrm{T}} \gamma_{\mu}^{\mathrm{T}}\right] \text {. }
$$


Using the property $\hat{C} \gamma_{\mu} \hat{C}^{-1}=-\gamma_{\mu}^{\mathrm{T}}$, where $\hat{C}=i \gamma^{2} \gamma^{0}$ represents the charge conjugation operator, and further permuting the last two factors $\gamma_{\mu} \hat{C}^{-1}$ under the trace to the left side, we convert the integral finally into the form

$$
-\int d^{4} \tilde{k} \frac{1}{(\tilde{k} \cdot q)} \operatorname{Tr}\left[\gamma_{\mu}\left(\not \not^{\prime}-\tilde{\not}-m\right) \gamma^{\xi}(\tilde{\not k}-\not h+m) \gamma_{\nu}(\tilde{\not}+m)\right] .
$$

The latter is, apart from the sign, identical to the second integral of Eq. (204) and therefore, the two integrals exactly cancel each other. This result of cancellation is, in general, known as the Furry's theorem. It states that Feynman diagrams containing a closed fermion loop with an odd number of photon vertices can be omitted in the calculation of physical processes [103]. It should be pointed out, however, that the cancellation of the interference terms happens only after integration over the momenta of the final leptons. These terms can still be accessed if one measures, for example, the angular distribution of the lepton pair.

\section{V.5 KINEMATICS}

As the Bjorken scaling variable $x_{B}$ tends to zero, both the Compton and Bethe-Heitler differential cross sections, see Eqs. (175) and (200), become singular. Nevertheless, the following kinematical constraint

$$
s-M_{\text {pair }}^{2} \geq\left(q-q^{\prime}+P\right)^{2} \geq M^{2}
$$

holds, where $M$ stands for the nucleon mass. Thus, in the laboratory frame, where the real and virtual photon momenta are $k=(\omega, \vec{k})$ and $k^{\prime}=\left(\omega^{\prime}, \vec{k}^{\prime}\right)$, respectively, and the nucleon momentum is $P=(M, \overrightarrow{0})$, the variable $x_{B}$ satisfies

$$
x_{B} \geq \frac{1}{1-\left(2 M \omega-M_{p a i r}^{2}\right) / t}
$$

For the invariant momentum transfer $t$ we have

$$
t=M_{p a i r}^{2}-2 \omega\left(\omega^{\prime}-\sqrt{\omega^{2}-M_{p a i r}^{2}} \cos \theta_{\gamma \gamma}\right)
$$

where $\theta_{\gamma \gamma}$ is the scattering angle, i.e. the angle between the directions of the initial and final photons.

We consider a proton target and the muons as the outgoing pair of leptons and thus we have $M \simeq 0.94 \mathrm{GeV}$ and $m \simeq 0.106 \mathrm{GeV}$. In Fig. 23, the Bjorken scaling 


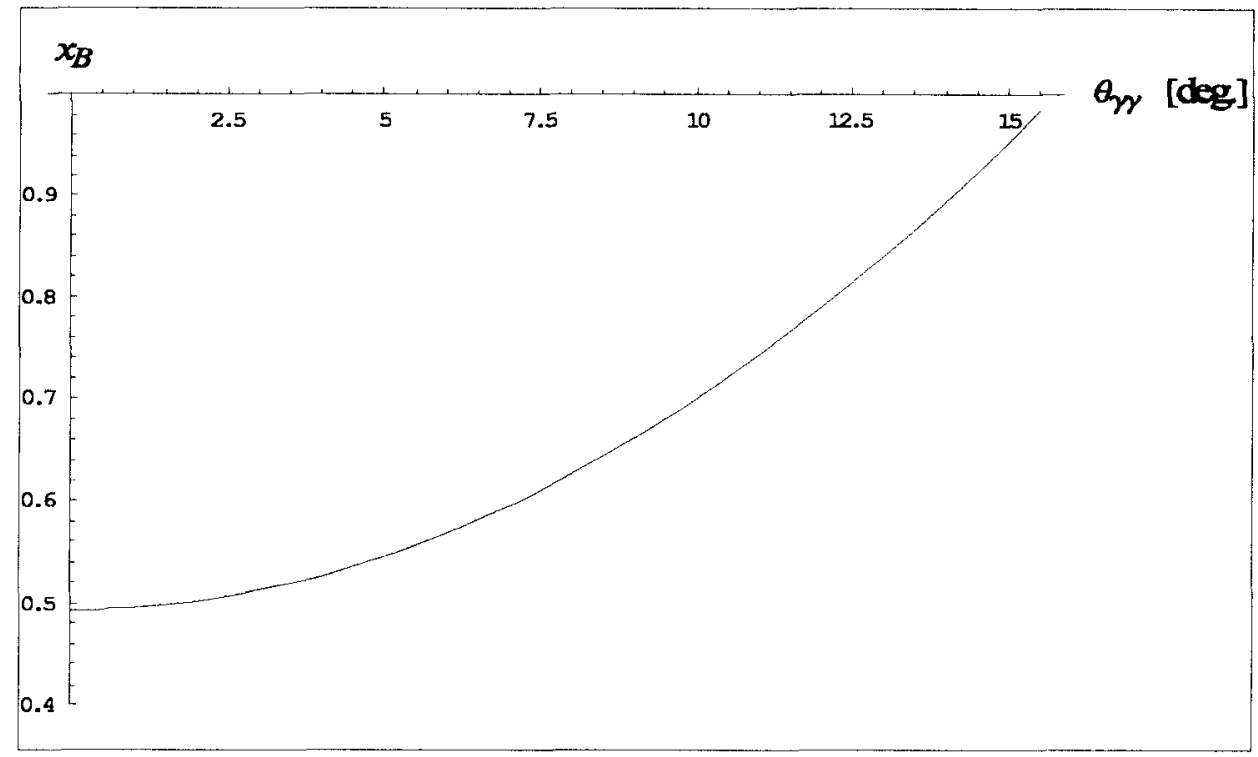

FIG. 23: Bjorken scaling variable $x_{B}$ plotted as a function of the angle $\theta_{\gamma \gamma}$ between the incoming real and outgoing virtual photon in the target rest frame for $\omega^{\prime}=10 \mathrm{GeV}$ and $M_{\text {pair }}=3 \mathrm{GeV}$ with $\omega=40 \mathrm{GeV}$ photon beam.

variable given by Eq. (174), is plotted against the angle $\theta_{\gamma \gamma}$ for fixed values of the incoming photon beam energy, $\omega=40 \mathrm{GeV}$, the energy of the outgoing virtual photon, $\omega^{\prime}=10 \mathrm{GeV}$, and the lepton-pair mass, $M_{\text {pair }}=3 \mathrm{GeV}$. We find $0.49 \leq$ $x_{B} \leq 0.96$ for the scattering angles $0 \leq \theta_{\gamma \gamma} \leq 15^{0}$. In this region of $x_{B}$, the valence quarks play the dominant role, in particular the $u$-flavor contribution, as shown in Fig. 25. Next we plot the angular dependence of the invariant $t$ for the same kinematics, see Fig. 24. Due to high energy of the photon beam, rather large values of $t$ are expected, namely, $27.85 \mathrm{GeV}^{2} \leq-t \leq 53.85 \mathrm{GeV}^{2}$. It is important to note that our description is not limited only to the small invariant momentum transfer, like in the case of DVCS kinematics discussed in Chapter IV. In contrary, it works for any kinematically allowed value of $t$.

To estimate the cross sections, we use a simplified parametrization of the unpolarized valence quark PDFs in the proton, given by Eq. (124), together with the sea quark distribution,

$$
\text { sea }(x)=0.5 x^{-0.75}(1-x)^{7}
$$

taken from Ref. [38]. These are, however, the valence distributions for the $u$ - and $d$-flavor components in the proton, respectively, and the sea distribution in the proton 


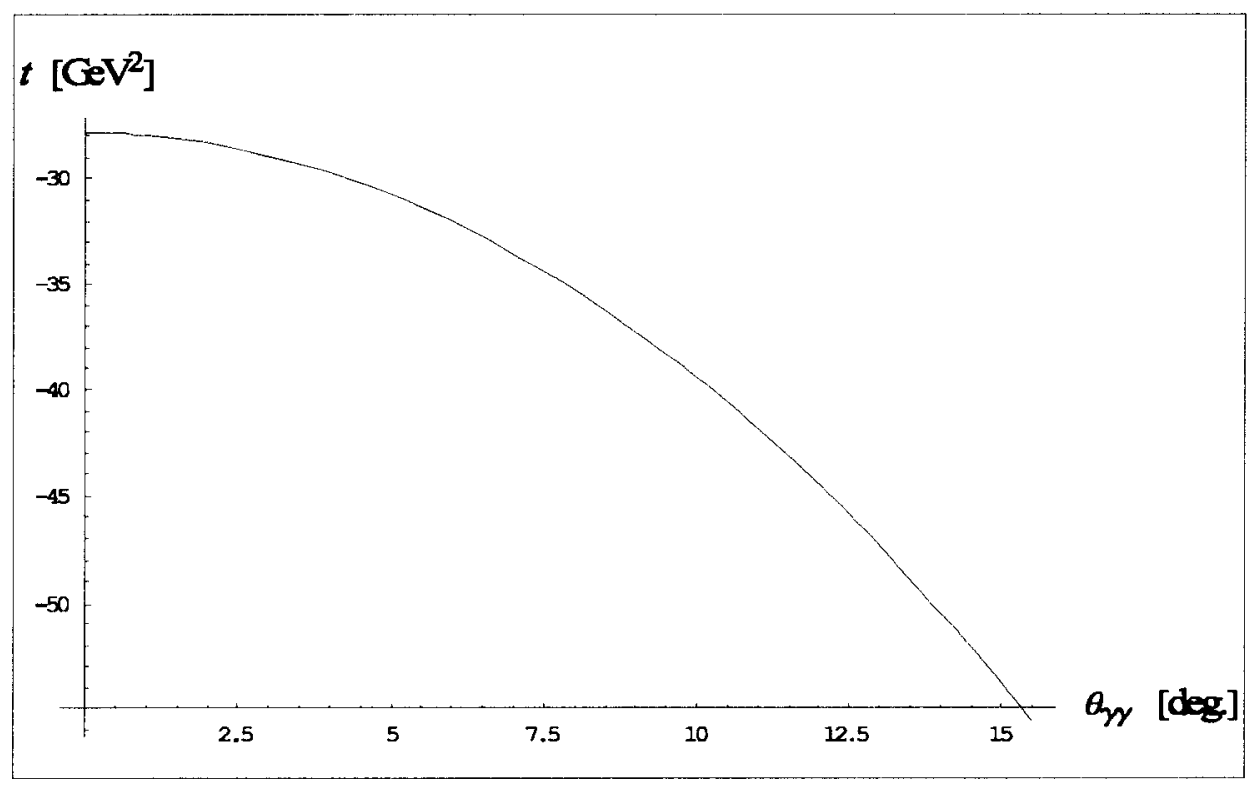

FIG. 24: Invariant momentum transfer $t$ plotted as a function of the angle $\theta_{\gamma \gamma}$ between the incoming real and outgoing virtual photon in the target rest frame for $\omega^{\prime}=10 \mathrm{GeV}$ and $M_{\text {pair }}=3 \mathrm{GeV}$ with $\omega=40 \mathrm{GeV}$ photon beam.

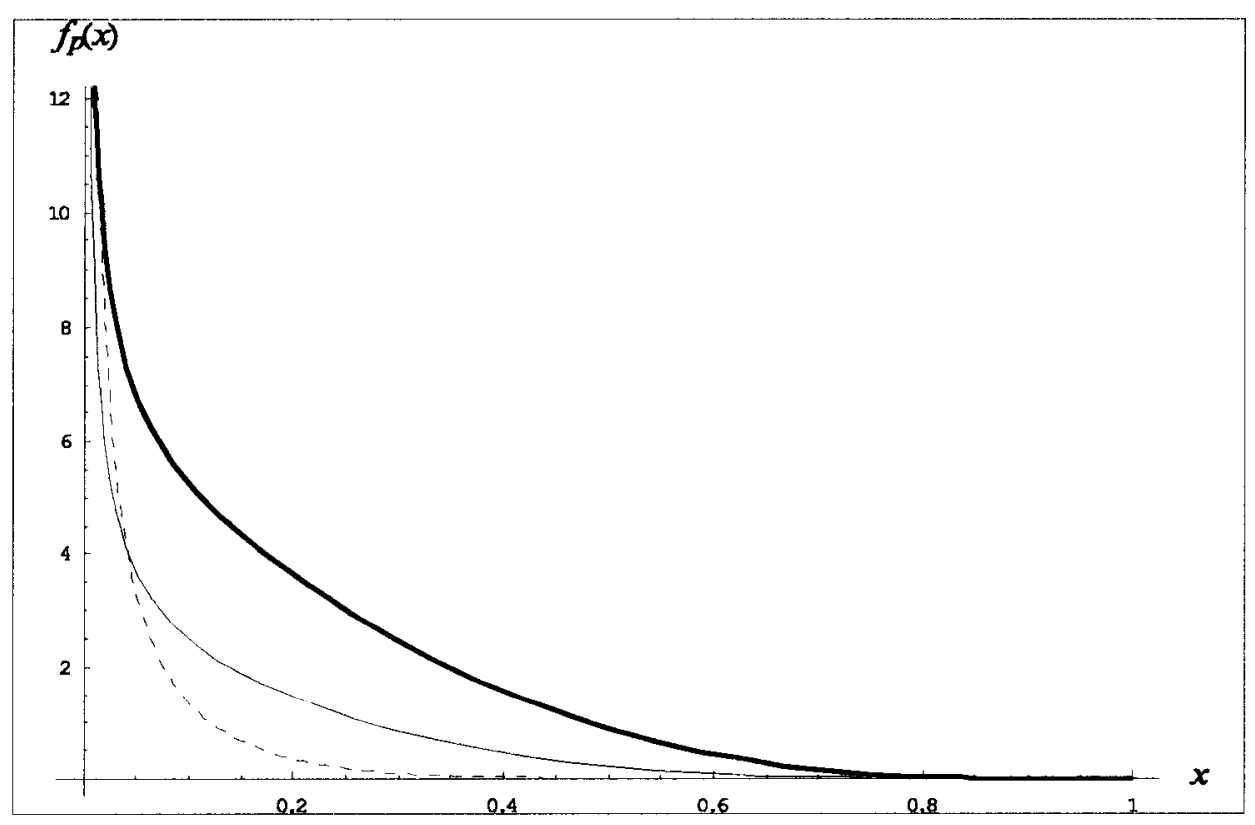

FIG. 25: Unpolarized valence quark distributions for $u$-flavor (bold solid line) and $d$-flavor (solid line), and the sea quark distribution (dashed line) in the proton. 


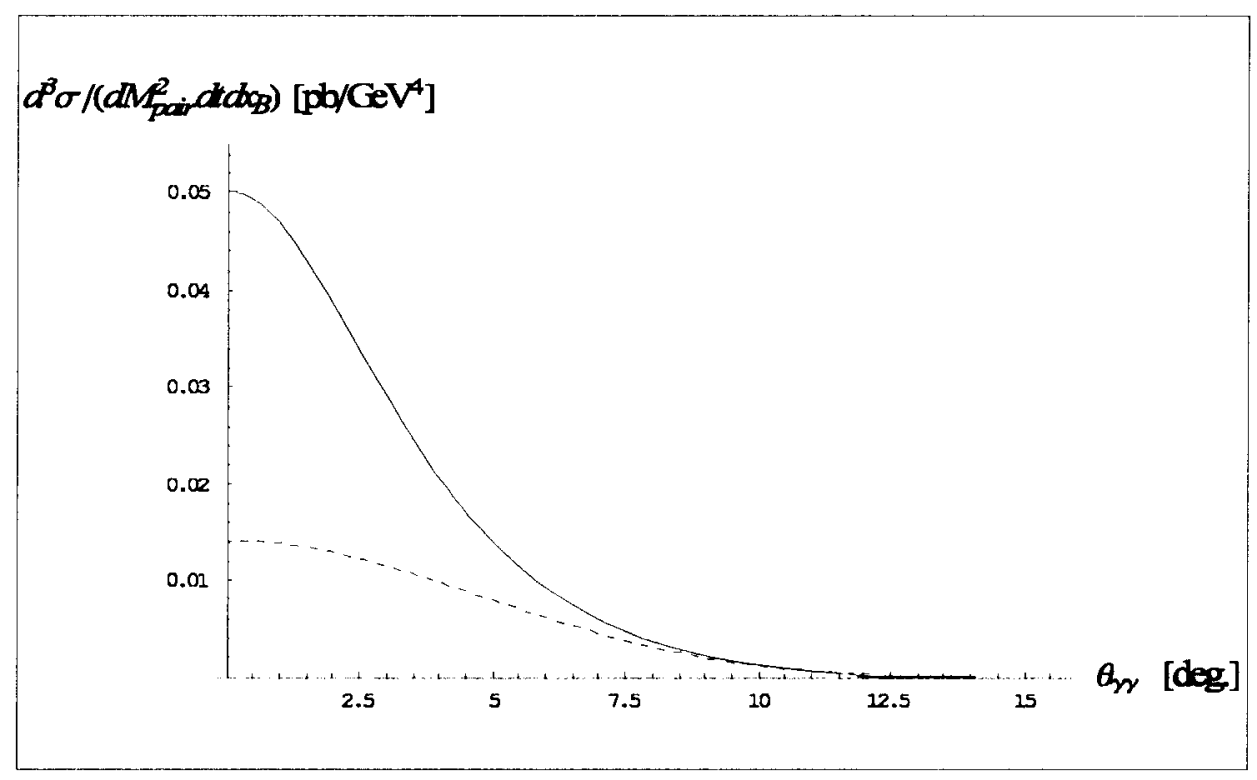

FIG. 26: Compton cross section $\sigma_{C}$ (solid line) and Bethe-Heitler cross section $\sigma_{B H}$ (dashed line) plotted as a function of the angle $\theta_{\gamma \gamma}$ between the incoming real and outgoing virtual photon in the target rest frame for $\omega^{\prime}=10 \mathrm{GeV}$ and $M_{\text {pair }}=3 \mathrm{GeV}$ with $\omega=40 \mathrm{GeV}$ photon beam.

whereas the distributions, which appear in the cross section formulas (175) and (200) are the quark and antiquark proton PDFs. In fact, what one really needs is the sum of the quark and antiquark distributions,

$$
\sum_{a} f_{a / p}(x)=\sum_{f}\left[f_{p}(x)+\bar{f}_{p}(x)\right] .
$$

As noted in Section IV.2, sum (211) corresponds to adding the valence PDFs and twice the contribution from sea quarks.

The unpolarized cross section results are presented in Fig. 26. We observe that the Compton contribution dominates by a factor $\simeq 3$ in the forward direction, i.e. for $\theta_{\gamma \gamma} \leq 3^{0}$, and falls off rapidly, even below the Bethe Heitler contribution for the scattering angles $\theta_{\gamma \gamma}>10^{\circ}$.

In summary, we have demonstrated the application of the QCD parton model to the high-energy photoproduction of lepton pairs. The Compton and Bethe-Heitler cross sections were estimated for a given simple parametrization of usual parton distributions in the proton. Without explicit calculation of the interference terms, we have shown their mutual cancellation, after being integrated over the final lepton momenta. 


\section{CHAPTER VI}

\section{EXCLUSIVE PHOTOPRODUCTION OF LEPTON PAIRS}

\section{VI.1 INTRODUCTION}

Deeply virtual Compton scattering is considered to be theoretically the cleanest and simplest process, in which GPDs can be accessed. We can now slightly modify the process, simply by interchanging the two photons at the subprocess level,

$$
\gamma\left(q_{1}\right)+N\left(p_{1}\right) \longrightarrow \gamma^{*}\left(q_{2}\right)+N\left(p_{2}\right)
$$

Then one arrives at time-like Compton scattering (TCS) [104] which is, in principle, the inverse process to DVCS. In a specific kinematical regime, where the virtuality of the final-state photon is large, $q_{2}^{2} \rightarrow \infty$, TCS becomes a handbag dominated process and accordingly, it can be studied in same way as DVCS was studied in Chapter IV.

Time-like Compton scattering can be accessed through the physical process known as the exclusive photoproduction of lepton pairs. At this point, we need to draw a distinction between the exclusive and the inclusive photoproduction of lepton pairs. In the former case we detect, in addition to the lepton pair, also the scattered nucleon $N$ (with the four-momentum $p_{2}$ ), as illustrated in Fig. 27. Another important difference is that unlike the inclusive photoproduction, we are limited now to small invariant momentum transfer $t \equiv\left(p_{1}-p_{2}\right)^{2}$ to the nucleon. Recall that the DVCS kinematics requires small $t$ compared to the invariant $q_{2}^{2}$ (the DVCS scale $Q_{1}^{2}$ is now replaced by the virtuality $\left.q_{2}^{2}\right)$, and thus the terms $\mathcal{O}\left(-t / q_{2}^{2}\right)$ in the amplitude can be safely dropped out. On the other hand, the common feature of both inclusive and exclusive photoproduction processes is the presence of two types of diagrams. In particular, for the exclusive photoproduction of lepton pairs, they are shown in Fig. 28.

In Section VI.2, we define the kinematics. In the next section, we compute the leading-twist amplitude for the Compton part. It can be easily derived from the result for VCA established in Section IV.2. The relevant integrals in the Compton and Bethe-Heitler cross sections can be evaluated simply by following the steps of Sections V.2 and V.3. Finally, the cross sections are estimated by using the same model for the nucleon GPDs as in the DVCS process. 


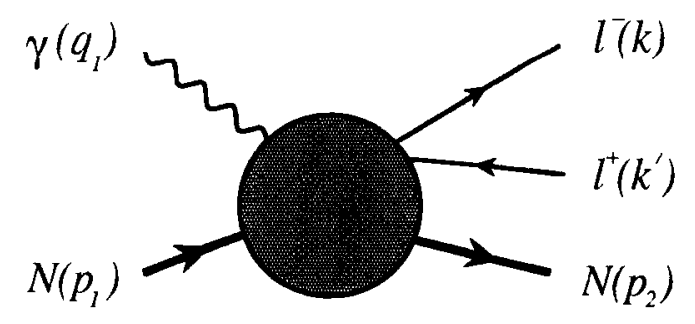

FIG. 27: Exclusive photoproduction of lepton pairs.

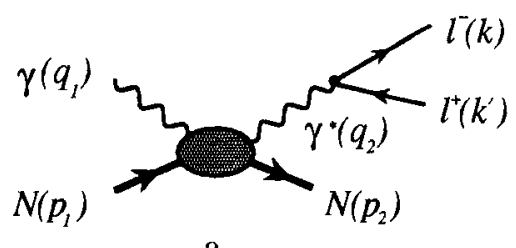

a
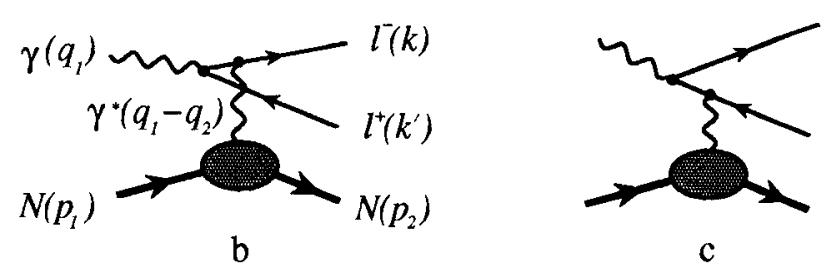

FIG. 28: DVCS (a) and Bethe-Heitler (b and c) diagrams contributing to the exclusive photoproduction of a lepton pair.

\section{VI.2 KINEMATICS}

In the laboratory reference frame, as the target rest frame, the photon and nucleon four-momenta are denoted by $q_{1}=\left(\nu_{1}, \vec{q}_{1}\right), q_{2}=\left(\nu_{2}, \vec{q}_{2}\right), p_{1}=(M, \overrightarrow{0})$ and $p_{2}=$ $\left(E_{2}, \vec{p}_{2}\right)$ where $M$ stands, as usual, for the nucleon mass. One rotates the coordinate system so that the $z$-axis is in the direction of the incident photon in the photon scattering plane, formed by the photon three-momenta $\vec{q}_{1}$ and $\vec{q}_{2}$. Similarly, the final leptons define the lepton scattering plane. We write their four-momenta as $k=(\omega, \vec{k})$ and $k^{\prime}=\left(\omega^{\prime}, \vec{k}^{\prime}\right)$, respectively. In this reference frame, the invariant momentum transfer can be expressed as a function of the angle $\theta_{\gamma \gamma}$ between the incoming real and outgoing virtual photon, namely,

$$
t \equiv\left(q_{2}-q_{1}\right)^{2}=q_{2}^{2}-2 \nu_{1}\left(\nu_{2}-\sqrt{\nu_{2}^{2}-q_{2}^{2}} \cos \theta_{\gamma \gamma}\right) .
$$

On the other hand, the invariant $t$ is also equal to

$$
t=2 M\left(\nu_{2}-\nu_{1}\right)
$$


which follows from the expression $t=2 M^{2}-2 M E_{2}$ and the energy conservation, $M+\nu_{1}=E_{2}+\nu_{2}$. Combining Eqs. (213) and (214) gives the quadratic equation in the energy of the virtual photon $\nu_{2}$ with the solutions

$$
\nu_{2}=\frac{A B \pm \sqrt{A^{2}-q_{2}^{2}\left(B^{2}-1\right)}}{B^{2}-1}
$$

The coefficients are

$$
\begin{aligned}
& A=\frac{(1+\chi) M}{\cos \theta_{\gamma \gamma}} \geq M, \\
& B=\frac{\left(1+M / \nu_{1}\right)}{\cos \theta_{\gamma \gamma}} \geq 1,
\end{aligned}
$$

where the scaling variable,

$$
\chi \equiv \frac{q_{2}^{2}}{2\left(p_{1} \cdot q_{1}\right)}=\frac{q_{2}^{2}}{2 M \nu_{1}}
$$

was introduced. It is an analog of the Bjorken scaling variable, $x_{B} \equiv q_{1}^{2} / 2\left(p_{1} \cdot q_{1}\right)$, in the DVCS process. Since in the DVCS kinematics the invariant momentum transfer $t$ is kept as small as possible, only the plus solution of Eq. (215) should be considered.

To illustrate the kinematics, we set the beam energy of initial photons to $\nu_{1}=$ $5 \mathrm{GeV}$, and the invariant mass of the lepton pair to $q_{2}^{2}=3 \mathrm{GeV}^{2}$. Accordingly, the scaling variable (217) is fixed at $\chi=0.32$. The invariant momentum is plotted as a function of the scattering angle in Fig. 29. It covers the range $0.15 \mathrm{GeV}^{2} \leq-t \leq$ $1.19 \mathrm{GeV}^{2}$ for $\theta_{\gamma \gamma}$ up to $10^{\circ}$. For the same region in $\theta_{\gamma \gamma}$, we also plot the energy $E_{2}$ of the final-state nucleon, see Fig 30 , and obtain $1.019 \mathrm{GeV} \leq E_{2} \leq 1.574 \mathrm{GeV}$.

\section{VI.3 CROSS SECTION}

In general, the differential cross section for the photoproduction of lepton pairs is

$$
d \sigma=\frac{1}{2 s}|\mathrm{~T}|^{2} \frac{1}{(2 \pi)^{5}} \delta^{(4)}\left(p_{1}+q_{1}-p_{2}-k-k^{\prime}\right) \frac{d^{3} p_{2}}{2 E_{2}} \frac{d^{3} k}{2 \omega} \frac{d^{3} k^{\prime}}{2 \omega^{\prime}}
$$

where the invariant $s \equiv\left(p_{1}+q_{1}\right)^{2}=2 M \nu_{1}+M^{2}$. Similarly to the DVCS case, the invariant matrix element consists of two contributions. Let us first investigate the Compton part. 


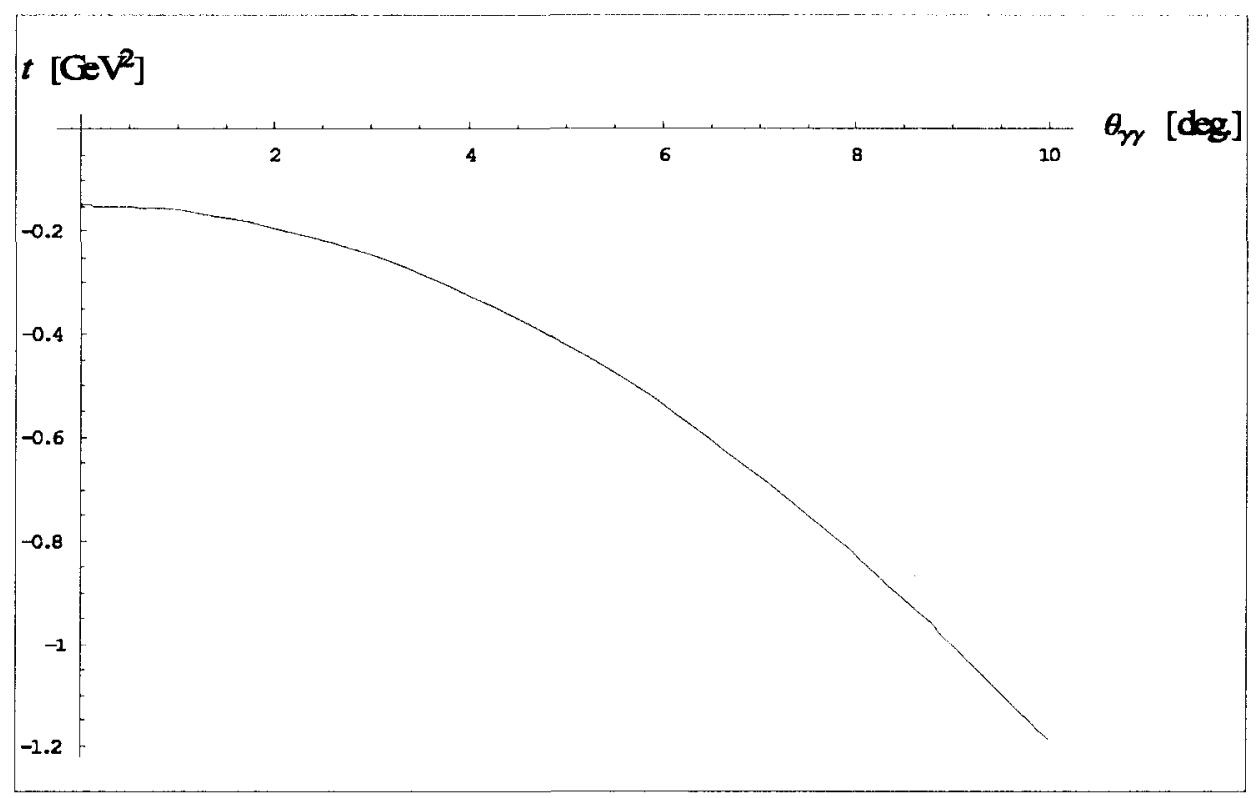

FIG. 29: Invariant momentum transfer $t$ plotted as a function of the angle $\theta_{\gamma \gamma}$ between the incoming real and outgoing virtual photon in the target rest frame for the invariant mass of the lepton pair $q_{2}^{2}=3 \mathrm{GeV}^{2}$ and $\chi=0.32$ with $\nu_{1}=5 \mathrm{GeV}$ photon beam.

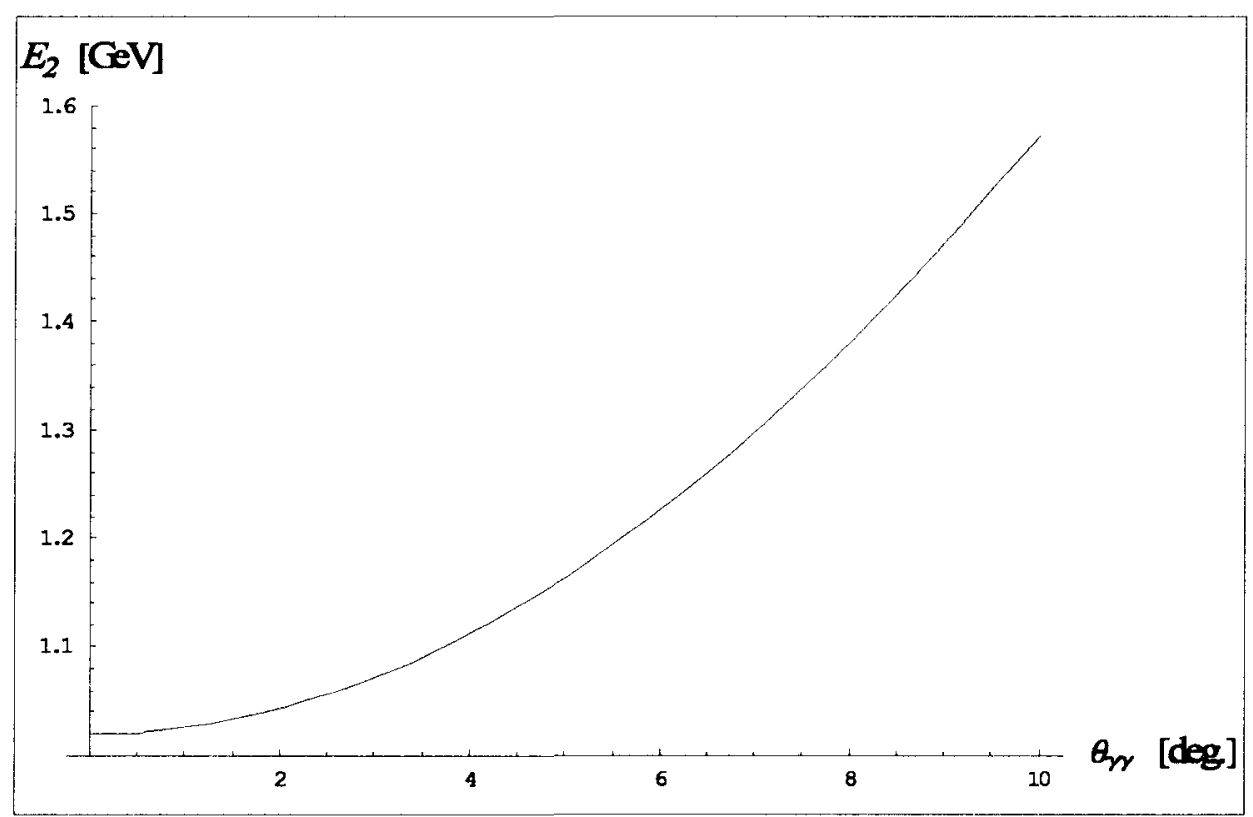

FIG. 30: Energy of the scattered nucleon $E_{2}$ plotted as a function of the angle $\theta_{\gamma \gamma}$ between the incoming real and outgoing virtual photon in the target rest frame for the invariant mass of the lepton pair $q_{2}^{2}=3 \mathrm{GeV}^{2}$ and $\chi=0.32$ with $\nu_{1}=5 \mathrm{GeV}$ photon beam. 


\section{VI.3.1 Compton Process}

The T-matrix of the Compton contribution is given by the diagram (a) in Fig. 28. Using $\epsilon_{\nu}\left(q_{1}\right)$ to denote the polarization of the initial photon, we have

$$
\mathrm{T}_{C}=\frac{|e|^{3}}{q_{2}^{2}} \bar{u}(k) \gamma_{\mu} v\left(k^{\prime}\right) \mathcal{T}_{T C S}^{\mu \nu} \epsilon_{\nu}\left(q_{1}\right),
$$

where $\mathcal{T}_{T C S}^{\mu \nu}$ is the reduced VCA for time-like Compton scattering, namely,

$$
\mathcal{T}_{T C S}^{\mu \nu}=i \int d^{4} z e^{i(q \cdot z)}\left\langle N\left(p_{2}, s_{2}\right)\left|T\left\{J_{E M}^{\mu}(z / 2) J_{E M}^{\nu}(-z / 2)\right\}\right| N\left(p_{1}, s_{1}\right)\right\rangle .
$$

In the twist-2 approximation, this amplitude is calculated from two ( $s$-and $u$-channel) handbag diagrams illustrated in Fig. 12. Unlike the standard electromagnetic DVCS process, the incoming photon is now real, $q_{1}^{2}=0$, and the outgoing photon is timelike, $q_{2}^{2}>0$. The final result for $\mathcal{T}_{T C S}^{\mu \nu}$ at the twist-2 level in terms of OFPDs can be derived from the expression (102) for the reduced VCA in the DVCS process simply by replacing the final photon momentum with the initial one, $q_{2} \rightarrow q_{1}$, and changing the sign in the skewness parameter $\eta$. Recall from Section IV.2 that the latter is defined as $\eta \equiv(r \cdot q) / 2(p \cdot q)$, where $r=p_{1}-p_{2}$ is the overall momentum transfer to the nucleon, and $p=\left(p_{1}+p_{2}\right) / 2$ and $q=\left(q_{1}+q_{2}\right) / 2$ are the average nucleon and photon momenta, respectively. As for DVCS $\eta=x_{B} /\left(2-x_{B}\right)$ in the DVCS kinematics, we have for TCS a similar relation, i.e. $\eta=\chi /(2-\chi)$. Moreover, the scaling variable $\xi \equiv-q^{2} / 2(p \cdot q)$ now coincides with $-\eta$.

Substituting $x \rightarrow-x$ and using the symmetry properties of OFPDs given by Eq. (97), we obtain the reduced VCA for TCS at the leading-twist level in the form

$$
\begin{array}{r}
\mathcal{T}_{\text {TCStwist-2 }}^{\mu \nu}=\frac{1}{2(p \cdot q)} \sum_{f} Q_{f}^{2} \int_{-1}^{1} \frac{d x}{(x-\eta-i 0)} \\
\times\left\{H_{f}^{+}(x, \eta, t)\left[\frac{1}{\left(p \cdot q_{1}\right)}\left(p^{\mu} q_{1}^{\nu}+p^{\nu} q_{1}^{\mu}\right)-g^{\mu \nu}\right] \bar{u}\left(p_{2}, s_{2}\right) q_{1} u\left(p_{1}, s_{1}\right)\right. \\
+E_{f}^{+}(x, \eta, t)\left[\frac{1}{\left(p \cdot q_{1}\right)}\left(p^{\mu} q_{1}^{\nu}+p^{\nu} q_{1}^{\mu}\right)-g^{\mu \nu}\right] \bar{u}\left(p_{2}, s_{2}\right) \frac{\left(d_{1} \not-\not q_{1}\right)}{4 M} u\left(p_{1}, s_{1}\right) \\
+\tilde{H}_{f}^{+}(x, \eta, t)\left[\frac{1}{\left(p \cdot q_{2}\right)} i \epsilon^{\mu \nu \rho \eta} q_{1 \rho} p_{\eta}\right] \bar{u}\left(p_{2}, s_{2}\right) q_{1} \gamma_{5} u\left(p_{1}, s_{1}\right) \\
\left.-\tilde{E}_{f}^{+}(x, \eta, t)\left[\frac{1}{\left(p \cdot q_{1}\right)} i \epsilon^{\mu \nu \rho \eta} q_{1 \rho} p_{\eta}\right] \frac{\left(q_{1} \cdot r\right)}{2 M} \bar{u}\left(p_{2}, s_{2}\right) \gamma_{5} u\left(p_{1}, s_{1}\right)\right\} .
\end{array}
$$

Since the photons in the amplitude (221) are interchanged compared to the standard VCA, the electromagnetic gauge invariance of $\mathcal{T}_{\text {TCStwist-2 }}^{\mu \nu}$ is now satisfied with respect 
to the initial photon, but violated in $\mathcal{O}\left(r^{2}\right)$ with respect to the final one. Similarly to the standard DVCS process, we introduce the following integrals

$$
\begin{aligned}
\mathcal{H}_{T C S}^{+}(\eta, t) & \equiv \sum_{f} Q_{f}^{2} \int_{-1}^{1} \frac{d x}{(x-\eta-i 0)} H_{f}^{+}(x, \eta, t), \\
\mathcal{E}_{T C S}^{+}(\eta, t) & \equiv \sum_{f} Q_{f}^{2} \int_{-1}^{1} \frac{d x}{(x-\eta-i 0)} H_{f}^{+}(x, \eta, t), \\
\tilde{\mathcal{H}}_{T C S}^{+}(\eta, t) & \equiv \sum_{f} Q_{f}^{2} \int_{-1}^{1} \frac{d x}{(x-\eta-i 0)} \tilde{H}_{f}^{+}(x, \eta, t), \\
\tilde{\mathcal{E}}_{T C S}^{+}(\eta, t) & \equiv \sum_{f} Q_{f}^{2} \int_{-1}^{1} \frac{d x}{(x-\eta-i 0)} \tilde{E}_{f}^{+}(x, \eta, t) .
\end{aligned}
$$

They can be calculated with the help of Eq. (103). For illustration, we plot in Figs. 31 and 32 the real and imaginary parts of the convolution integral $\mathcal{H}_{T C S}^{+}(\eta, t)=\mathcal{H}_{T C S(u)}^{+}(\eta, t)+\mathcal{H}_{T C S(d)}^{+}(\eta, t)$. We present the $u$ - and $d$-quark contributions separately, using a GPD model from Section IV.4. To get rid of the $t$-dependence, we divide by appropriate factors, $F_{1 u}(t) / 2$ and $F_{1 d}(t)$. Analogous plots for $\tilde{\mathcal{H}}_{T C S}^{+}(\eta, t)=\tilde{\mathcal{H}}_{T C S(u)}^{+}(\eta, t)+\tilde{\mathcal{H}}_{T C S(d)}^{+}(\eta, t)$ (here we divide by the factor $g_{A}(t) / g_{A}(t=0)$ to remove the dependence on $\left.t\right)$, are given in Figs. 33 and 34. All four figures clearly show that, for the proton target, the valence $u$-quark contribution to the real and imaginary parts of the integrals $\mathcal{H}_{T C S}^{+}(\eta, t)$ and $\tilde{\mathcal{H}}_{T C S}^{+}(\eta, t)$ is much larger compared to the valence $d$-quark contribution.

We will restrict ourselves to the unpolarized incoming photon beam and the unpolarized nucleon target. Then the spin-averaged square of the T-matrix in the factorized form is

$$
\overline{\left|\mathrm{T}_{C}\right|^{2}}=\frac{(4 \pi \alpha)^{3}}{2 q_{2}^{4}} L_{\mu \alpha}^{C} H_{C}^{\mu \alpha}
$$

where the lepton and hadron tensors are

$$
\begin{aligned}
L_{\mu \alpha}^{C}= & 4\left[k_{\mu} k_{\alpha}^{\prime}+k_{\alpha} k_{\mu}^{\prime}-g_{\mu \alpha}\left[\left(k \cdot k^{\prime}\right)+m^{2}\right]\right] \\
H_{C}^{\mu \alpha}= & -\frac{1}{2} \mathcal{T}_{T C S}^{\mu \nu}\left(\mathcal{T}_{\nu T C S}^{\alpha}\right)^{*} \\
= & {\left[\left(1-\eta^{2}\right)\left(\left|\mathcal{H}_{T C S}^{+}\right|^{2}+\left|\tilde{\mathcal{H}}_{T C S}^{+}\right|^{2}\right)\right.} \\
& -\eta^{2}\left(\mathcal{H}_{T C S}^{+*} \mathcal{E}_{T C S}^{+}+\mathcal{H}_{T C S}^{+} \mathcal{E}_{T C S}^{+*}+\tilde{\mathcal{H}}_{T C S}^{+*} \tilde{\mathcal{E}}_{T C S}^{+}+\tilde{\mathcal{H}}_{T C S}^{+} \tilde{\mathcal{E}}_{T C S}^{+*}\right) \\
& \left.-\left(\eta^{2}+\frac{t}{4 M^{2}}\right)\left|\mathcal{E}_{T C S}^{+}\right|^{2}-\eta^{2} \frac{t}{4 M^{2}}\left|\tilde{\mathcal{E}}_{T C S}^{+}\right|^{2}\right] \\
& \times\left[\frac{1}{\left(p \cdot q_{1}\right)}\left(p^{\mu} q_{1}^{\alpha}+p^{\alpha} q_{1}^{\mu}\right)-\frac{M^{2}}{\left(p \cdot q_{1}\right)^{2}}\left(1-\frac{t}{4 M^{2}}\right) q_{1}^{\mu} q_{1}^{\alpha}-g^{\mu \alpha}\right]
\end{aligned}
$$




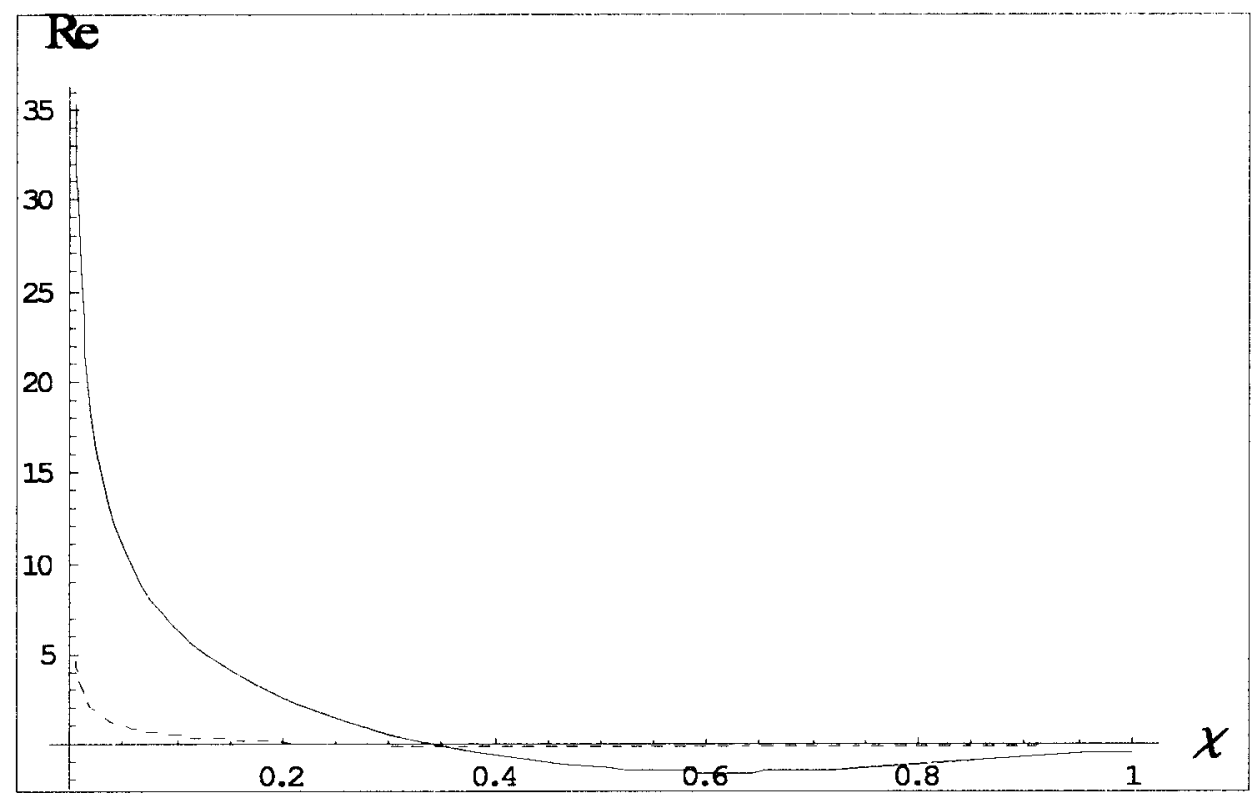

FIG. 31: Contributions from $u$ quarks (solid line) and $d$ quarks (dashed line) to the real part of $\mathcal{H}_{T C S}^{+}$. They are divided by $F_{1 u}(t) / 2$ and $F_{1 d}(t)$, respectively.

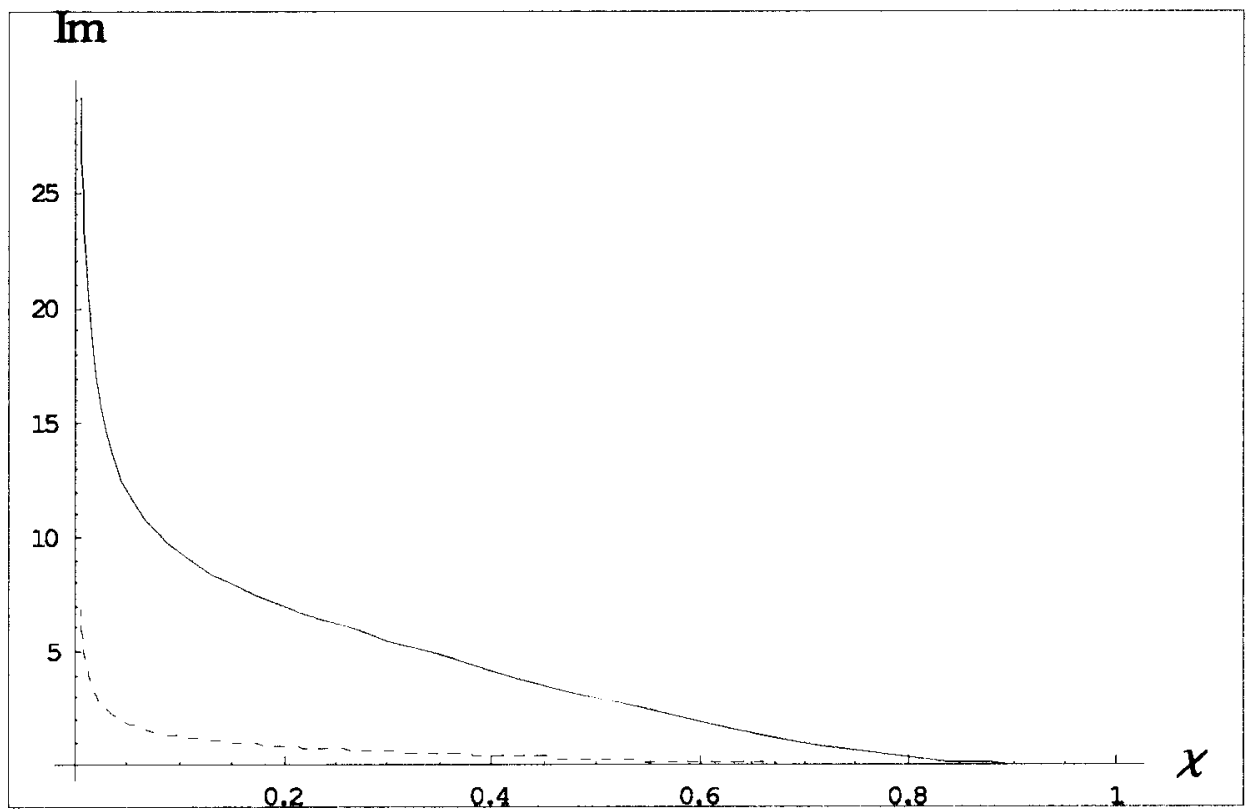

FIG. 32: Contributions from $u$ quarks (solid line) and $d$ quarks (dashed line) to the imaginary part of $\mathcal{H}_{T C S}^{+}$. They are divided by $F_{1 u}(t) / 2$ and $F_{1 d}(t)$, respectively. 


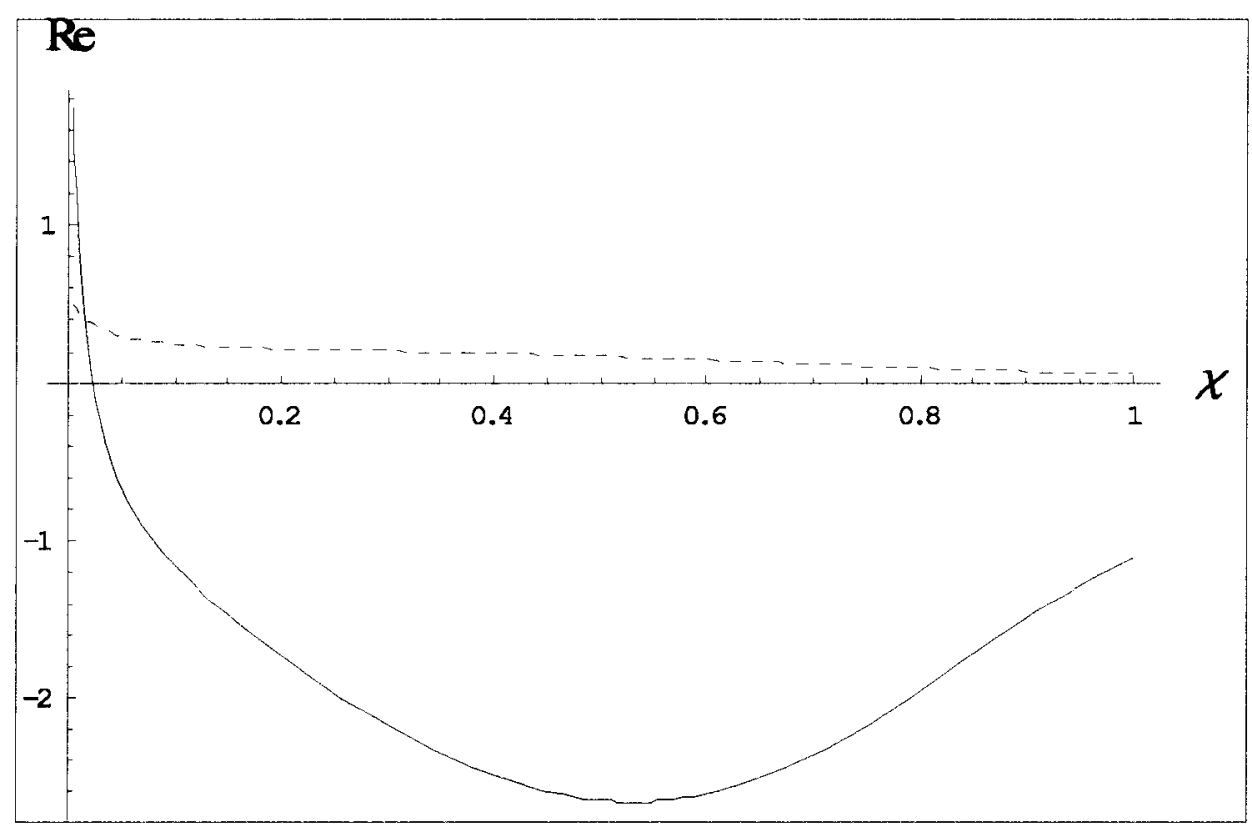

FIG. 33: Contributions from $u$ quarks (solid line) and $d$ quarks (dashed line) to the real part of $\tilde{\mathcal{H}}_{T C S}^{+}$divided by $g_{A}(t) / g_{A}(t=0)$.

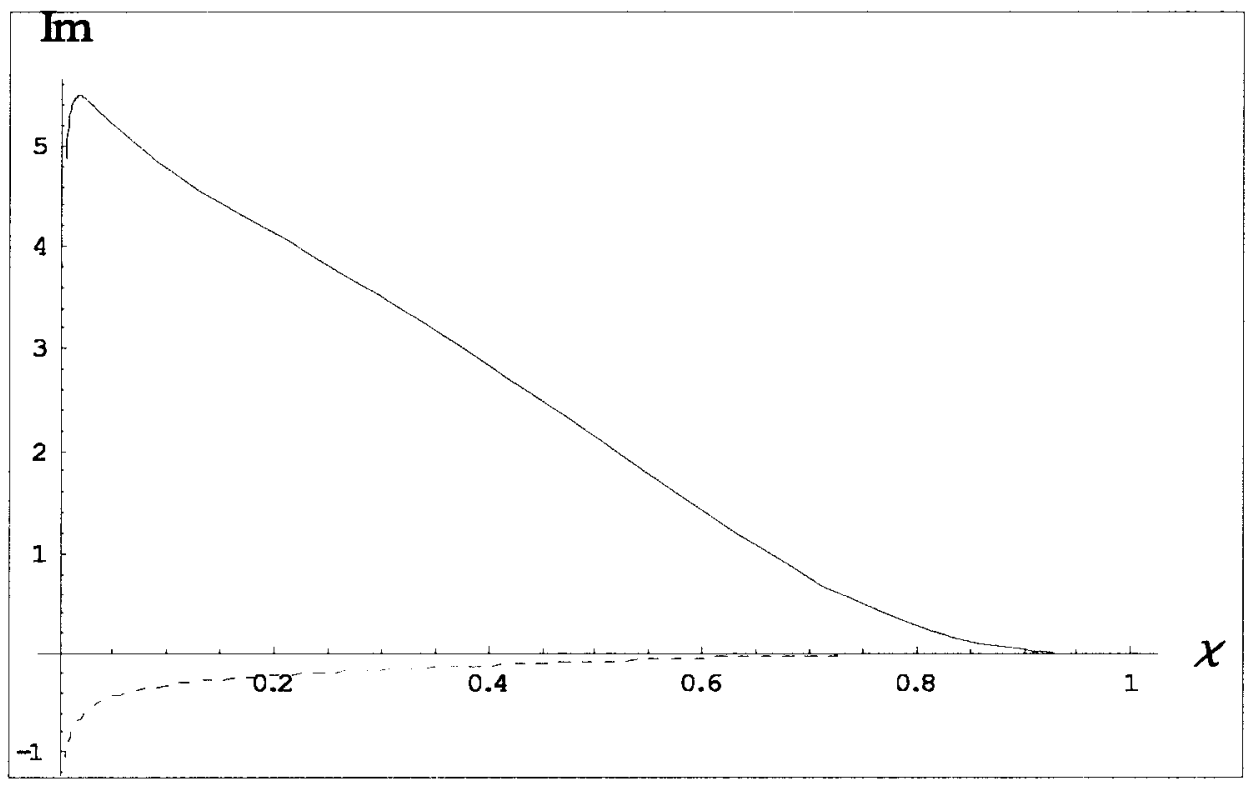

FIG. 34: Contributions from $u$ quarks (solid line) and $d$ quarks (dashed line) to the imaginary part of $\tilde{\mathcal{H}}_{T C S}^{+}$divided $g_{A}(t) / g_{A}(t=0)$. 
with $m$ denoting the lepton mass. Note that $H_{C}^{\mu \alpha}$ has the same structure as its DVCS partner given by the expression (138). To calculate the cross section, we integrate Eq. (223) over the Lorentz-invariant phase space. This is done exactly in the same way as in Section V.2 for the case of the inclusive photoproduction of lepton pairs. Thus first we integrate $L_{\mu \alpha}^{C}$ over the part of the phase space associated with both final leptons,

$$
\begin{array}{r}
\int \frac{d^{3} k}{2 \omega} \int \frac{d^{3} k^{\prime}}{2 \omega^{\prime}} \delta^{(4)}\left(p_{1}+q_{1}-\right. \\
\left.p_{2}-k-k^{\prime}\right) L_{\mu \alpha}^{C}=\int d^{4} q_{2} \delta^{(4)}\left(p_{1}+q_{1}-p_{2}-q_{2}\right) \\
\times\left[q_{2}^{2} g_{\mu \alpha}-q_{2 \mu} q_{2 \alpha}\right]\left(-\frac{2 \pi}{3} \frac{q_{2}^{2}+2 m^{2}}{q_{2}^{2}} \sqrt{1-\frac{4 m^{2}}{q_{2}^{2}}}\right),
\end{array}
$$

and then contract the tensor $\left[q_{2}^{2} g_{\mu \alpha}-q_{2 \mu} q_{2 \alpha}\right]$ with $H_{C}^{\mu \alpha}$. It is easy to see that, after contraction, the first term is of the order $q_{2}^{2}$ while the second term is $\mathcal{O}\left(M^{2}\right)$. The latter can be therefore ignored in the limit $M^{2} / q_{2}^{2} \rightarrow 0$. We are still left with two more integrations. One of them, e.g. integration over $\vec{p}_{2}$, is trivial due to the presence of the delta function $\delta^{(4)}\left(p_{1}+q_{1}-p_{2}-q_{2}\right)$ on the right-hand side of Eq. (225). The cross section now turns into

$$
\sigma_{C}=-\frac{\alpha^{3}}{3 \pi} \frac{q_{2}^{2}+2 m^{2}}{s q_{2}^{4}} \sqrt{1-\frac{4 m^{2}}{q_{2}^{2}}} \int d^{4} q_{2} \delta^{+}\left[\left(p_{1}+q_{1}-q_{2}\right)^{2}-M^{2}\right]\left[g_{\mu \alpha} H_{C}^{\mu \alpha}\right],
$$

where the contraction in the square brackets reads

$$
\begin{aligned}
g_{\mu \alpha} H_{C}^{\mu \alpha}= & -2\left[\left(1-\eta^{2}\right)\left(\left|\mathcal{H}_{T C S}^{+}\right|^{2}+\left|\tilde{\mathcal{H}}_{T C S}^{+}\right|^{2}\right)\right. \\
& -\eta^{2}\left(\mathcal{H}_{T C S}^{+*} \mathcal{E}_{T C S}^{+}+\mathcal{H}_{T C S}^{+} \mathcal{E}_{T C S}^{+*}+\tilde{\mathcal{H}}_{T C S}^{+*} \tilde{\mathcal{E}}_{T C S}^{+}+\tilde{\mathcal{H}}_{T C S}^{+} \tilde{\mathcal{E}}_{T C S}^{+*}\right) \\
& \left.-\left(\eta^{2}+\frac{t}{4 M^{2}}\right)\left|\mathcal{E}_{T C S}^{+}\right|^{2}-\eta^{2} \frac{t}{4 M^{2}}\left|\tilde{\mathcal{E}}_{T C S}^{+}\right|^{2}\right] .
\end{aligned}
$$

The remaining integral in Eq. (226) is performed in the photon-nucleon center-ofmass-frame, in which the nucleon mass can be safely neglected. The final result for the unpolarized Compton differential cross section in the invariant form is

$$
\frac{d^{2} \sigma_{C}}{d M_{p a i r}^{2} d t}=-\frac{\alpha^{3}}{6} \frac{M_{p a i r}^{2}+2 m^{2}}{s^{2} M_{p a i r}^{4}} \sqrt{1-\frac{4 m^{2}}{M_{p a i r}^{2}}}\left[g_{\mu \alpha} H_{C}^{\mu \alpha}\right]
$$

\section{VI.3.2 Bethe-Heitler Process}

The Bethe-Heitler amplitude,

$$
\mathrm{T}_{B H}=\frac{|e|^{3}}{t} \epsilon_{\nu}\left(q_{1}\right) \bar{u}(k)\left[\frac{\gamma^{\mu} q_{1} \gamma^{\nu}-2 \gamma^{\mu} k^{\prime \nu}}{-2\left(k^{\prime} \cdot q_{1}\right)}+\frac{-\gamma^{\nu} q_{1} \gamma^{\mu}+2 k^{\nu} \gamma^{\mu}}{-2\left(k \cdot q_{1}\right)}\right] v\left(k^{\prime}\right)
$$




$$
\times\left\langle N\left(p_{2}, s_{2}\right)\left|J_{\mu}(0)\right| N\left(p_{1}, s_{1}\right)\right\rangle,
$$

comes from the diagrams (b) and (c) in Fig. 28. Dirac and Pauli nucleon form factors parametrize the nucleon transition current matrix element,

$$
\left\langle N\left(p_{2}, s_{2}\right)\left|J_{\mu}(0)\right| N\left(p_{1}, s_{1}\right)\right\rangle=\bar{u}\left(p_{2}, s_{2}\right)\left[F_{1}(t) \gamma_{\mu}-F_{2}(t) \frac{i \sigma_{\mu \lambda} r^{\lambda}}{2 M}\right] u\left(p_{1}, s_{1}\right) .
$$

After averaging and summing $\left|\mathrm{T}_{B H}\right|^{2}$ over the polarizations of the initial and final particles, respectively, one has

$$
\overline{\left|\mathrm{T}_{B H}\right|^{2}}=\frac{(4 \pi \alpha)^{3}}{2 t^{2}} L_{B H}^{\mu \alpha} H_{\mu \alpha}^{B H},
$$

where both tensors have already been calculated. The hadron tensor is obtained by replacing the Lorentz indices $\nu \rightarrow \mu$ and $\beta \rightarrow \alpha$ in the expression (144),

$$
\begin{aligned}
H_{\mu \alpha}^{B H}= & t\left[g_{\mu \alpha}-\frac{r_{\mu} r_{\alpha}}{t}\right]\left[F_{1}(t)+F_{2}(t)\right]^{2} \\
& +4\left[p_{1 \mu}+\frac{r_{\mu}}{2}\right]\left[p_{1 \alpha}+\frac{r_{\alpha}}{2}\right]\left[F_{1}^{2}(t)-\frac{t}{4 M^{2}} F_{2}^{2}(t)\right],
\end{aligned}
$$

and the lepton tensor by replacing the momenta $q \rightarrow q_{1}$ and indices $\rho \rightarrow \alpha$ in Eq. (179),

$$
\begin{aligned}
L_{B H}^{\mu \alpha}= & 4\left\{\frac { 1 } { ( k \cdot q _ { 1 } ) ^ { 2 } } \left[\left(k \cdot q_{1}\right)\left[k^{\prime \mu} q_{1}^{\alpha}+k^{\prime \alpha} q_{1}^{\mu}-g^{\mu \alpha}\left(k^{\prime} \cdot q_{1}\right)\right]\right.\right. \\
& -m^{2}\left[k^{\mu} k^{\prime \alpha}+k^{\alpha} k^{\prime \mu}-g^{\mu \alpha}\left(k \cdot k^{\prime}\right)\right] \\
& +m^{2}\left[k^{\prime \mu} q_{1}^{\alpha}+k^{\prime \alpha} q_{1}^{\mu}-g^{\mu \alpha}\left(k^{\prime} \cdot q_{1}\right)\right] \\
& \left.-m^{2}\left(k \cdot q_{1}\right) g^{\mu \alpha}+m^{4} g^{\mu \alpha}\right] \\
& +\frac{1}{\left(k^{\prime} \cdot q_{1}\right)^{2}}\left[\left(k^{\prime} \cdot q_{1}\right)\left[k^{\mu} q_{1}^{\alpha}+k^{\alpha} q_{1}^{\mu}-g^{\mu \alpha}\left(k \cdot q_{1}\right)\right]\right. \\
& -m^{2}\left[k^{\mu} k^{\prime \alpha}+k^{\alpha} k^{\prime \mu}-g^{\mu \alpha}\left(k \cdot k^{\prime}\right)\right] \\
& +m^{2}\left[k^{\mu} q_{1}^{\alpha}+k^{\alpha} q_{1}^{\mu}-g^{\nu \alpha}\left(k \cdot q_{1}\right)\right] \\
& \left.-m^{2}\left(k^{\prime} \cdot q_{1}\right) g^{\mu \alpha}+m^{4} g^{\mu \alpha}\right] \\
& +\frac{1}{\left(k \cdot q_{1}\right)\left(k^{\prime} \cdot q_{1}\right)}\left[2\left(k \cdot k^{\prime}\right)\left[k^{\mu} k^{\prime \alpha}+k^{\alpha} k^{\prime \mu}-g^{\mu \alpha}\left(k \cdot k^{\prime}\right)\right]\right. \\
& -\left(k \cdot q_{1}\right)\left[k^{\mu} k^{\prime \alpha}+k^{\alpha} k^{\prime \mu}-2 k^{\prime \mu} k^{\prime \alpha}\right] \\
& -\left(k^{\prime} \cdot q_{1}\right)\left[k^{\mu} k^{\prime \alpha}+k^{\alpha} k^{\prime \mu}-2 k^{\mu} k^{\alpha}\right] \\
& -\left(k \cdot k^{\prime}\right)\left[k^{\mu} q_{1}^{\alpha}+k^{\alpha} q_{1}^{\mu}+k^{\prime \mu} q_{1}^{\alpha}+k^{\prime \alpha} q_{1}^{\mu}\right] \\
& \left.\left.+2 g^{\mu \alpha}\left(k \cdot k^{\prime}\right)\left[\left(k \cdot q_{1}\right)+\left(k^{\prime} \cdot q_{1}\right)\right]-m^{2}\right]-2 m^{2} q_{1}^{\mu} q_{1}^{\alpha}\right\} .
\end{aligned}
$$


The integral of $L_{B H}^{\mu \alpha}$ over the final lepton momenta is known from Section V.3, namely,

$$
\begin{aligned}
\mathcal{L}_{B H}^{\mu \alpha} & \equiv \int \frac{d^{3} k}{2 \omega} \int \frac{d^{3} k^{\prime}}{2 \omega^{\prime}} \delta^{(4)}\left(q_{2}-k-k^{\prime}\right) L_{B H}^{\mu \alpha} \\
& =\frac{2 \pi}{\left(q_{1} \cdot q_{2}\right)}\left[\mathcal{A}_{B H} q_{1}^{\mu} q_{1}^{\alpha}+\mathcal{B}_{B H} q_{2}^{\mu} q_{2}^{\alpha}+\mathcal{C}_{B H}\left(q_{1}^{\mu} q_{2}^{\alpha}+q_{2}^{\mu} q_{1}^{\alpha}\right)+\mathcal{D}_{B H} g^{\mu \alpha}\right]
\end{aligned}
$$

where

$$
\begin{aligned}
& \mathcal{A}_{B H}=\left[\frac{2 q_{2}^{2}}{\left(q_{1} \cdot q_{2}\right)}-\frac{2 q_{2}^{4}}{\left(q_{1} \cdot q_{2}\right)^{2}}+\frac{q_{2}^{6}}{\left(q_{1} \cdot q_{2}\right)^{3}}-\frac{8 m^{2} q_{2}^{2}}{\left(q_{1} \cdot q_{2}\right)^{2}}-\frac{4 m^{4} q_{2}^{2}}{\left(q_{1} \cdot q_{2}\right)^{3}}+\frac{6 m^{2} q_{2}^{4}}{\left(q_{1} \cdot q_{2}\right)^{3}}\right. \\
& \left.+\frac{4 m^{2}}{\left(q_{1} \cdot q_{2}\right)}\right] \ln \left[\frac{1-\sqrt{1-4 m^{2} / q_{2}^{2}}}{1+\sqrt{1-4 m^{2} / q_{2}^{2}}}\right] \\
& +\left[\frac{4 q_{2}^{2}}{\left(q_{1} \cdot q_{2}\right)}-\frac{8 q_{2}^{4}}{\left(q_{1} \cdot q_{2}\right)^{2}}+\frac{4 q_{2}^{6}}{\left(q_{1} \cdot q_{2}\right)^{3}}+\frac{2 m^{2} q_{2}^{4}}{\left(q_{1} \cdot q_{2}\right)^{3}}\right] \sqrt{1-\frac{4 m^{2}}{q_{2}^{2}}} \\
& \mathcal{B}_{B H}=\frac{4 m^{2}}{\left(q_{1} \cdot q_{2}\right)} \ln \left[\frac{1-\sqrt{1-4 m^{2} / q_{2}^{2}}}{1+\sqrt{1-4 m^{2} / q_{2}^{2}}}\right]+\frac{2 q_{2}^{2}}{\left(q_{1} \cdot q_{2}\right)} \sqrt{1-\frac{4 m^{2}}{q_{2}^{2}}} \\
& \mathcal{C}_{B H}=\left[-2+\frac{2 q_{2}^{2}}{\left(q_{1} \cdot q_{2}\right)}-\frac{q_{2}^{4}}{\left(q_{1} \cdot q_{2}\right)^{2}}-\frac{6 m^{2} q_{2}^{2}}{\left(q_{1} \cdot q_{2}\right)^{2}}+\frac{4 m^{2}}{\left(q_{1} \cdot q_{2}\right)}+\frac{4 m^{4}}{\left(q_{1} \cdot q_{2}\right)^{2}}\right] \\
& \times \ln \left[\frac{1-\sqrt{1-4 m^{2} / q_{2}^{2}}}{1+\sqrt{1-4 m^{2} / q_{2}^{2}}}\right] \\
& +\left[-2+\frac{6 q_{2}^{2}}{\left(q_{1} \cdot q_{2}\right)}-\frac{4 q_{2}^{4}}{\left(q_{1} \cdot q_{2}\right)^{2}}-\frac{2 m^{2} q_{2}^{2}}{\left(q_{1} \cdot q_{2}\right)^{2}}\right] \sqrt{1-\frac{4 m^{2}}{q_{2}^{2}}}, \\
& \mathcal{D}_{B H}=\left[2\left(q_{1} \cdot q_{2}\right)-2 q_{2}^{2}+\frac{q_{2}^{4}}{\left(q_{1} \cdot q_{2}\right)}+\frac{2 m^{2} q_{2}^{2}}{\left(q_{1} \cdot q_{2}\right)}-\frac{4 m^{4}}{\left(q_{1} \cdot q_{2}\right)}\right] \\
& \times \ln \left[\frac{1-\sqrt{1-4 m^{2} / q_{2}^{2}}}{1+\sqrt{1-4 m^{2} / q_{2}^{2}}}\right] \\
& +\left[2\left(q_{1} \cdot q_{2}\right)-4 q_{2}^{2}+\frac{2 q_{2}^{4}}{\left(q_{1} \cdot q_{2}\right)}+\frac{2 m^{2} q_{2}^{2}}{\left(q_{1} \cdot q_{2}\right)}\right] \sqrt{1-\frac{4 m^{2}}{q_{2}^{2}}} .
\end{aligned}
$$

The unpolarized Bethe-Heitler cross section is then

$$
\sigma_{B H}=\frac{1}{2 s} \frac{1}{(2 \pi)^{5}} \frac{(4 \pi \alpha)^{3}}{2 t^{2}} \frac{\pi}{2}\left(\frac{s-M_{p a i r}^{2}}{2 s}\right) \int d M_{p a i r}^{2} \int d\left(\cos \theta_{c m}\right)\left[\mathcal{L}_{B H}^{\mu \alpha} H_{\mu \alpha}^{B H}\right],
$$

and finally in the differential invariant form,

$$
\frac{d^{2} \sigma_{B H}}{d M_{p a i r}^{2} d t}=\frac{\alpha^{3}}{4 \pi} \frac{1}{s^{2} t^{2}}\left[\mathcal{L}_{B H}^{\mu \alpha} H_{\mu \alpha}^{B H}\right]
$$


The contraction in the square brackets of Eq. (236) gives

$$
\begin{aligned}
\mathcal{L}_{B H}^{\mu \alpha} H_{\mu \alpha}^{B H}= & \frac{4 \pi}{M_{\text {pair }}^{2}-t}\left\{\left[-\frac{\mathcal{A}_{B H}}{4}\left(1-\frac{t}{M_{\text {pair }}^{2}}\right)^{2}+\mathcal{B}_{B H}\left[\frac{t}{M_{\text {pair }}^{2}}-\frac{1}{4}\left(1+\frac{t}{M_{\text {pair }}^{2}}\right)^{2}\right]\right.\right. \\
& \left.+\mathcal{C}_{B H}\left[\frac{t}{M_{\text {pair }}^{2}}\left(1-\frac{t}{M_{\text {pair }}^{2}}\right)-\frac{1}{2}\left(1-\left(\frac{t}{M_{\text {pair }}^{2}}\right)^{2}\right)\right]+3 \mathcal{D}_{B H} \frac{t}{M_{\text {pair }}^{4}}\right] \\
& \times M_{\text {pair }}^{4}\left[F_{1}(t)+F_{2}(t)\right]^{2}+\left[\mathcal{A}_{B H}\left[1-\frac{M^{2}}{s}+\frac{M_{\text {pair }}^{2}}{2 s}\left(1-\frac{t}{M_{\text {pair }}^{2}}\right)\right]^{2}\right. \\
& +\mathcal{B}_{B H}\left[1+\frac{t}{s}-\frac{M^{2}}{s}+\frac{M_{\text {pair }}^{2}}{2 s}\left(1+\frac{t}{M_{\text {pair }}^{2}}\right)\right]^{2} \\
& +2 \mathcal{C}_{B H}\left[1-\frac{M^{2}}{s}+\frac{M_{\text {pair }}^{2}}{2 s}\left(1-\frac{t}{M_{\text {pair }}^{2}}\right)\right] \\
& \times\left[1+\frac{t}{s}-\frac{M^{2}}{s}+\frac{M_{\text {pair }}^{2}}{2 s}\left(1+\frac{t}{M_{\text {pair }}^{2}}\right)\right] \\
& \left.\left.+4 \mathcal{D}_{B H} \frac{M^{2}}{s^{2}}\left(1+\frac{3 t}{4 M^{2}}\right)\right] s^{2}\left[F_{1}^{2}(t)-\frac{t}{4 M^{2}} F_{2}^{2}(t)\right]\right\} .
\end{aligned}
$$

Note that $q_{2}^{2}$ and $\left(q_{1} \cdot q_{2}\right)$, which appear in the coefficients (235), should be replaced by $M_{\text {pair }}^{2}$ and $\left(M_{\text {pair }}^{2}-t\right) / 2$, respectively.

In Figs. 35 and 36, we plot the Compton and Bethe-Heitler contributions using a toy model for the proton valence OFPDs described in Section IV.4. Both cross sections are shown together on a logarithmic scale in Fig. 37. We notice that, similarly to the standard DVCS, the Bethe-Heitler process is again the dominating contribution (i.e. $\simeq 0.95$ ) to the cross section.

We conclude with the remark that the cross sections presented here were integrated over the angles of the final-state leptons. One may expect to get additional information of GPDs, by measuring the cross sections that are sensitive to the angular distribution of final leptons. In particular, the interference terms between the Compton and Bethe-Heitler processes, together with the use of the polarized photon beam, may provide a new insight. This is an interesting subject by itself, and requires more attention in the future. 


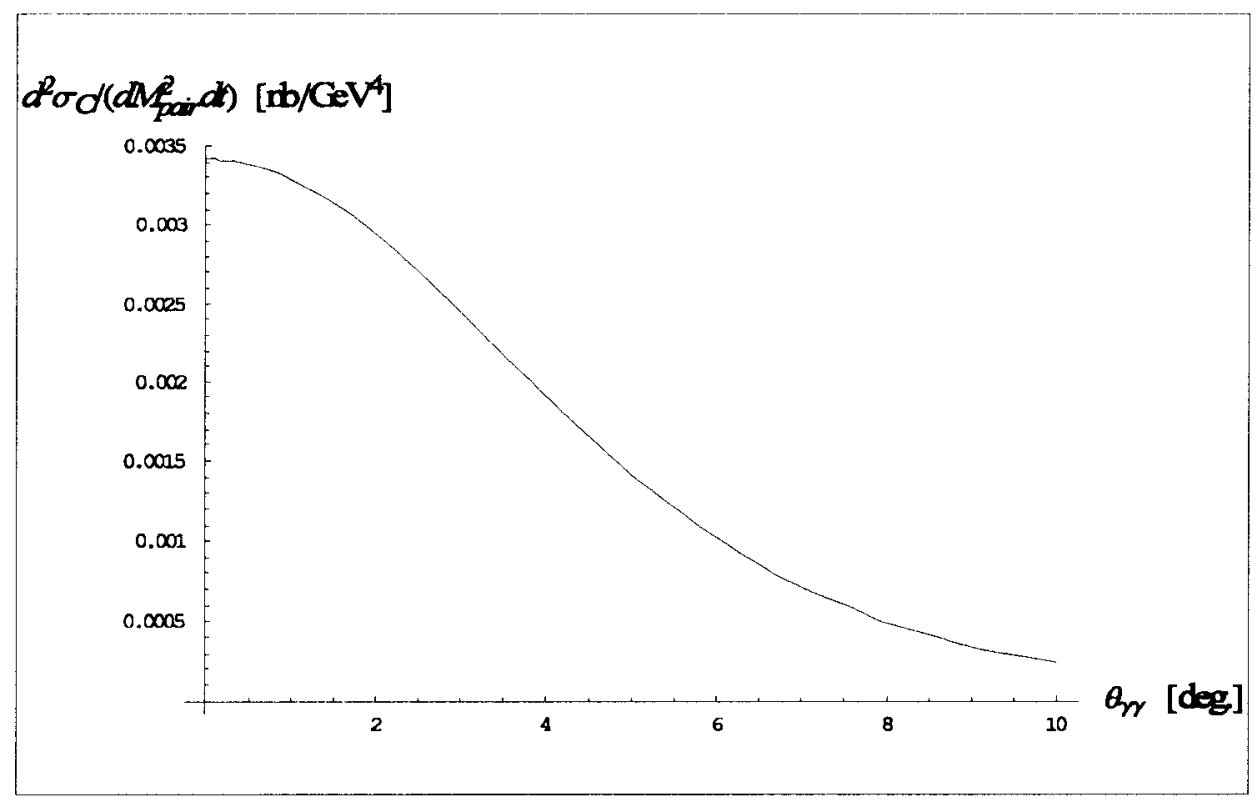

FIG. 35: Compton cross section $\sigma_{C}$ plotted as a function of the angle $\theta_{\gamma \gamma}$ between the incoming real and outgoing virtual photon in the target rest frame for $M_{\text {pair }}^{2}=3 \mathrm{GeV}^{2}$ and $\chi=0.32$ with $\nu_{1}=5 \mathrm{GeV}$ photon beam.

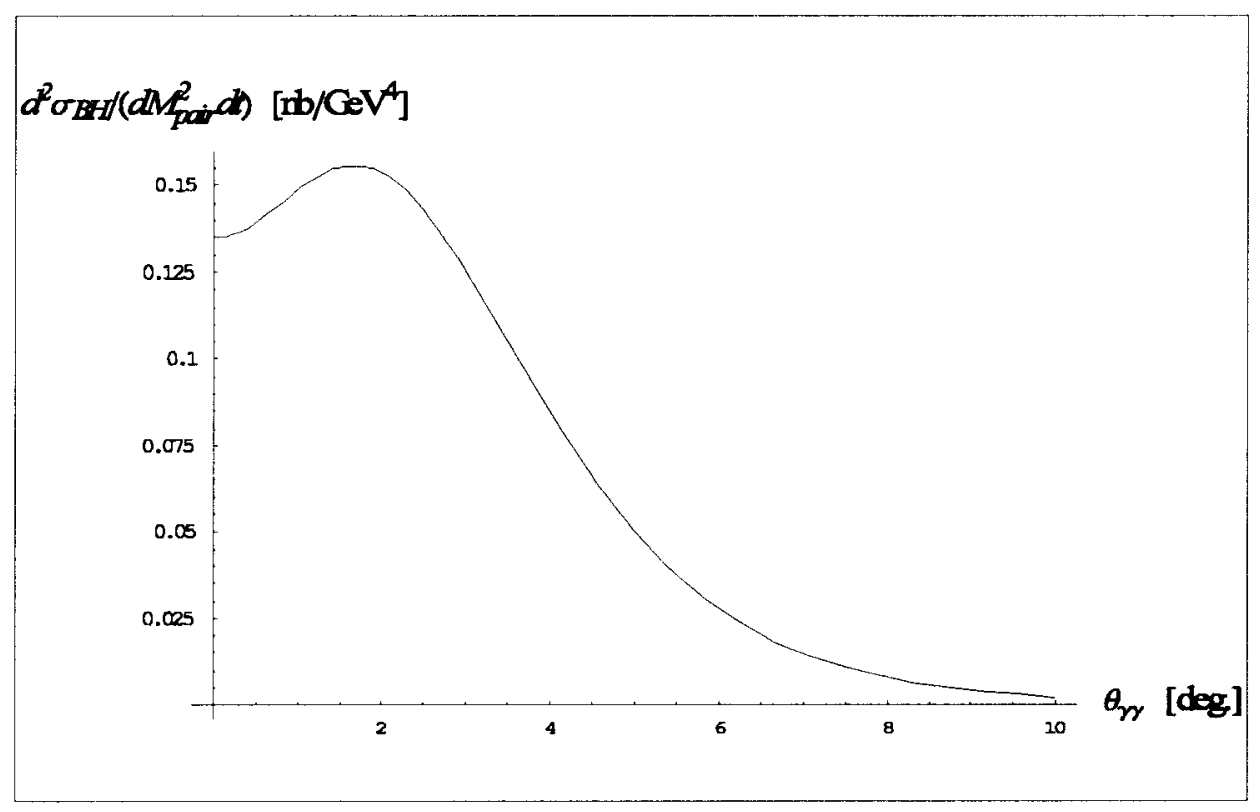

FIG. 36: Bethe-Heitler cross section $\sigma_{B H}$ plotted plotted as a function of the angle $\theta_{\gamma \gamma}$ between the incoming real and outgoing virtual photon in the target rest frame for $M_{\text {pair }}^{2}=3 \mathrm{GeV}^{2}$ and $\chi=0.32$ with $\nu_{1}=5 \mathrm{GeV}$ photon beam. 


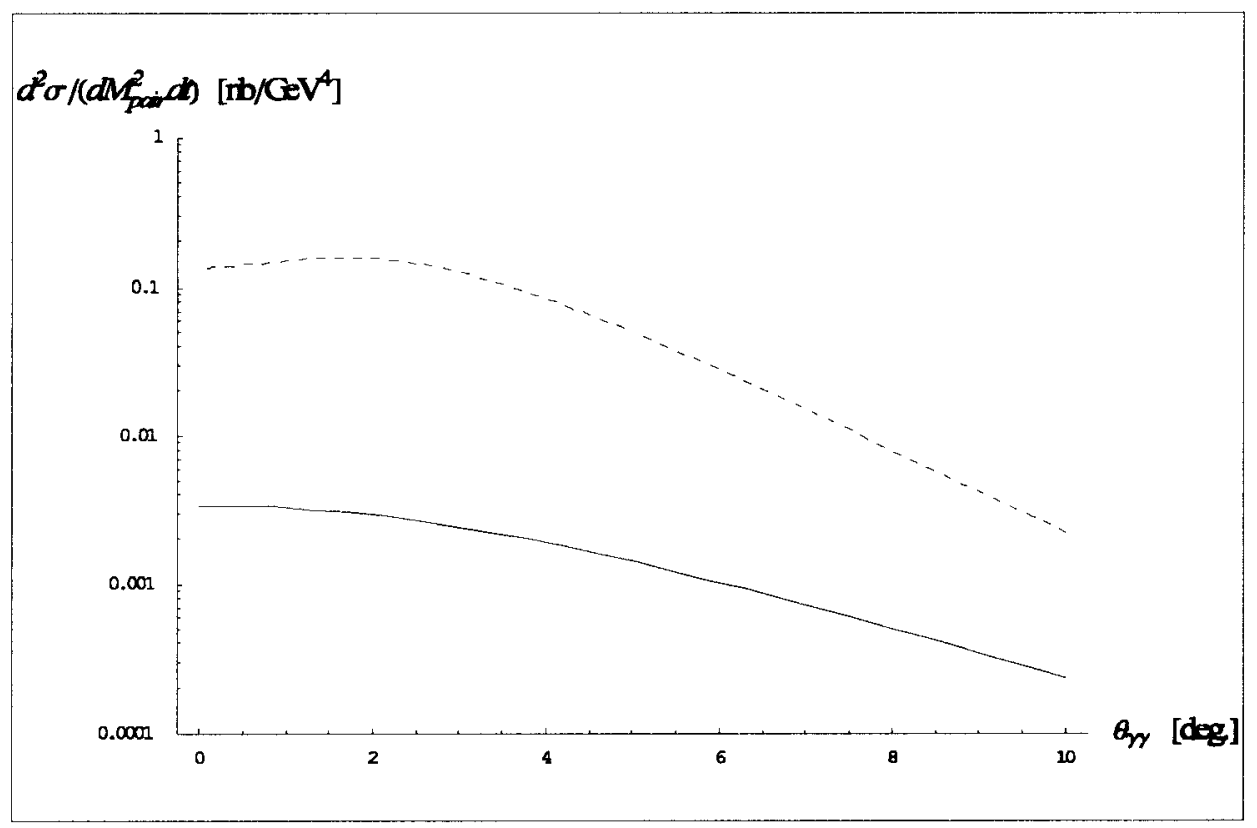

FIG. 37: Compton cross section $\sigma_{C}$ (solid line) and Bethe-Heitler cross section $\sigma_{B H}$ (dashed line) plotted as a function of the angle $\theta_{\gamma \gamma}$ between the incoming real and outgoing virtual photon in the target rest frame for $M_{\text {pair }}^{2}=3 \mathrm{GeV}^{2}$ and $\chi=0.32$ with $\nu_{1}=5 \mathrm{GeV}$ photon beam. 


\section{CHAPTER VII}

\section{WEAK DEEPLY VIRTUAL COMPTON SCATTERING}

\section{VII.1 INTRODUCTION}

Complementary to the standard electromagnetic DVCS process, different combinations of quark flavors can be accessed by utilizing the weak current, which couples to quarks with strengths proportional to the quark weak charges. In analogy with DVCS, these can be studied in neutrino-induced virtual Compton scattering,

$$
\nu(k)+N\left(p_{1}\right) \longrightarrow \nu\left(k^{\prime}\right)+N\left(p_{2}\right)+\gamma\left(q_{2}\right)
$$

for the neutral current, and

$$
\nu(k)+N\left(p_{1}\right) \longrightarrow \mu^{ \pm}\left(k^{\prime}\right)+N^{\prime}\left(p_{2}\right)+\gamma\left(q_{2}\right)
$$

for the charged current reactions (and similarly for antineutrinos). Note that in the case of the latter the initial and final nucleons will be different. Because of the $V$ - $A$ nature of the weak interactions, one can probe $\hat{C}$-odd combinations of GPDs as well as $\hat{C}$-even, where $\hat{C}$ is the charge conjugation operator, and thus measure independently both the valence and sea content of GPDs. The weak current also allows one to study flavor nondiagonal GPDs, such as those associated with the neutron-to-proton transitions in charged current reactions in Eq. (240). The use of weak currents can thus provide an important tool to complement the study of GPDs in more familiar electron-induced DVCS or exclusive meson production processes.

In this chapter, we study the weak deeply virtual Compton scattering processes given by Eqs. (240) and (239), and present a comprehensive account of the amplitudes and cross sections in the kinematics relevant to future high-intensity neutrino experiments [105]. In Section VII.2, we derive both the weak neutral and weak charged VCAs using the nonlocal light-cone OPE, and introduce an appropriate set of off-forward parton distributions, which parametrize the weak DVCS reactions. The weak DVCS processes are analyzed in Section VII.3. Using a simple model for nucleon OFPDs from Section IV.4 (recall that the model does not include the sea quark contribution), we estimate the cross sections and compare the respective rates in neutrino scattering with those in the standard DVCS process. In addition, for the sake 
of completeness, we also discuss the electron-induced DVCS process associated with the exchange of the weak boson $Z^{0}$.

Deeply virtual neutrino scattering has been discussed recently in Ref. [106] for neutral currents and in Ref. [107] for charged currents. Also a preliminary report containing some of the formal results from Ref. [109] appeared in Ref. [108].

\section{VII.2 WEAK VIRTUAL COMPTON SCATTERING AMPLITUDE}

In this section, we discuss the amplitudes for the weak virtual Compton scattering process. Before turning to the specific amplitudes for the neutral and charged current cases, we review some general aspects that have been already discussed in Chapter IV.

At the subprocess level, in analogy with the photon-induced DVCS amplitude, the weak virtual Compton scattering amplitude can be obtained by simply replacing the incoming virtual photon with the weak boson $B$,

$$
B\left(q_{1}\right)+N\left(p_{1}\right) \longrightarrow \gamma\left(q_{2}\right)+N^{\prime}\left(p_{2}\right)
$$

where $B$ is either $Z^{0}$ or $W^{ \pm}$. Note that in the electromagnetic and weak neutral cases both the incoming and outgoing nucleons are the same, $N=N^{\prime}$. Similarly to the electromagnetic VCA introduced in Section IV.2, for the weak process with an incoming $W^{ \pm}$or $Z^{0}$ boson and outgoing photon, one has

$$
\begin{aligned}
T_{W}^{\mu \nu}= & i \int d^{4} x \int d^{4} y e^{-i\left(q_{1} \cdot x\right)+i\left(q_{2} \cdot y\right)} \\
& \times\left\langle N^{\prime}\left(p_{2}, s_{2}\right)\left|T\left\{J_{E M}^{\mu}(y) J_{W}^{\nu}(x)\right\}\right| N\left(p_{1}, s_{1}\right)\right\rangle,
\end{aligned}
$$

where $J_{W}^{\nu}$ corresponds either to the weak neutral current or the weak charged current. We will denote the currents by $J_{W N}^{\nu}$ and $J_{W C}^{\nu}$, respectively. Again, due to the current conservation

$$
T_{W}^{\mu \nu} q_{1 \nu}=0 \quad \text { and } \quad q_{2 \mu} T_{W}^{\mu \nu}=0
$$

In terms of symmetric coordinates, $X \equiv(x+y) / 2$ and $z \equiv y-x$, and symmetric momentum variables, $q \equiv\left(q_{1}+q_{2}\right) / 2$ and $p \equiv\left(p_{1}+p_{2}\right) / 2$, the weak VCA takes the following form

$$
T_{W}^{\mu \nu}=(2 \pi)^{4} \delta^{(4)}\left(p_{1}+q_{1}-p_{2}-q_{2}\right) \mathcal{T}_{W}^{\mu \nu}
$$



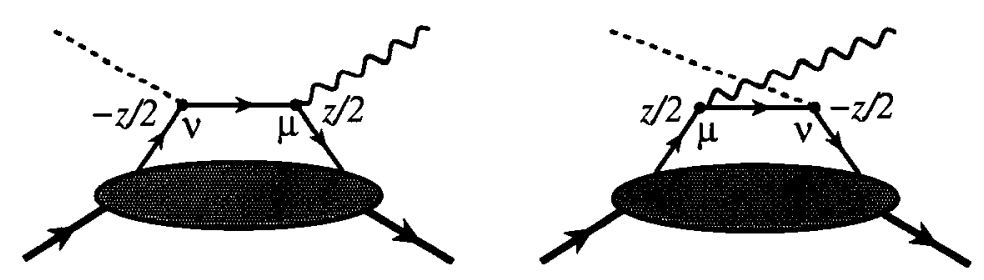

FIG. 38: Handbag diagrams ( $s$ - and $u$-channel) in the weak virtual Compton scattering amplitude.

with the expression for the reduced weak VCA

$$
\mathcal{T}_{W}^{\mu \nu}=i \int d^{4} z e^{i(q \cdot z)}\left\langle N^{\prime}\left(p-r / 2, s_{2}\right)\left|T\left\{J_{E M}^{\mu}(z / 2) J_{W}^{\nu}(-z / 2)\right\}\right| N\left(p+r / 2, s_{1}\right)\right\rangle
$$

Recall that, in general, the initial and final nucleons may be different, $p_{1}^{2}=M_{1}^{2}$ and $p_{2}^{2}=M_{2}^{2}$. Accordingly, Eq. (81) is now replaced by

$$
p^{2}=\frac{1}{2}\left(M_{1}^{2}+M_{2}^{2}-t / 2\right) \text { and }(p \cdot r)=\frac{1}{2}\left(M_{1}^{2}-M_{2}^{2}\right)
$$

however, it is recovered by neglecting the mass difference between the proton and neutron (we set $M_{1}=M_{2} \equiv M$ ).

Our starting point is the coordinate representation of the time-ordered product of the weak and electromagnetic currents. The leading light-cone singularity is contained in the handbag contribution illustrated in Fig. 38. Note that the weak current couples to the quark fields through two types of vertices, $q q Z^{0}$ and $q q W^{ \pm}$. Therefore, the quark fields at coordinates $\pm z / 2$ can carry either the same or different flavor quantum numbers. We study these two cases separately.

\section{VII.2.1 Weak Neutral Amplitude}

We start with the expansion of the time-ordered product of the weak neutral and electromagnetic current. Apart from an overall factor $-|e| g / \cos \theta_{W}$ (recall that $g$ is the coupling constant of the weak interaction), we have

$$
\begin{aligned}
i T\left\{J_{E M}^{\mu}(z / 2) J_{W N}^{\nu}(-z / 2)\right\}= & i \sum_{f} Q_{f}\left\{\bar{\psi}_{f}(z / 2) \gamma^{\mu} i \not s(z) \gamma^{\nu}\left(\frac{c_{V}^{f}-\gamma_{5} c_{A}^{f}}{2}\right)\right. \\
& \times \psi_{f}(-z / 2)+\bar{\psi}_{f}(-z / 2) \gamma^{\nu}\left(\frac{c_{V}^{f}-\gamma_{5} c_{A}^{f}}{2}\right) \\
& \left.\times i \not(-z) \gamma^{\mu} \psi_{f}(z / 2)\right\}
\end{aligned}
$$


where $c_{V}^{f}$ and $c_{A}^{f}$ are the weak vector and axial vector charges, respectively. They are given in the standard model by

$$
\begin{aligned}
c_{V}^{u, c, t} & =1 / 2-2 Q_{u, c, t} \sin ^{2} \theta_{W} \\
c_{V}^{d, s, b} & =-1 / 2-2 Q_{d, s, b} \sin ^{2} \theta_{W} \\
c_{A}^{u, c, t} & =1 / 2 \\
c_{A}^{d, s, b} & =-1 / 2
\end{aligned}
$$

where $\theta_{W}$ is the Weinberg angle with $\sin ^{2} \theta_{W} \simeq 0.23$. After substituting the explicit expression for the free quark propagator in the coordinate representation, see Eq. (88), and using the formula (89), the expansion (247) can be written in a compact form in terms of the string operators

$$
\begin{aligned}
i T\left\{J_{E M}^{\mu}(z / 2) J_{W N}^{\nu}(-z / 2)\right\}= & \frac{z_{\rho}}{4 \pi^{2} z^{4}} \sum_{f} Q_{f} \\
& \times\left\{c _ { V } ^ { f } \left[s^{\mu \rho \nu \eta}\left[\bar{\psi}_{f}(-z / 2) \gamma_{\eta} \psi_{f}(z / 2)-(z \rightarrow-z)\right]\right.\right. \\
& \left.+i \epsilon^{\mu \rho \nu \eta}\left[\bar{\psi}_{f}(-z / 2) \gamma_{\eta} \gamma_{5} \psi_{f}(z / 2)+(z \rightarrow-z)\right]\right] \\
& -c_{A}^{f}\left[s^{\mu \rho \nu \eta}\left[\bar{\psi}_{f}(-z / 2) \gamma_{\eta} \gamma_{5} \psi_{f}(z / 2)-(z \rightarrow-z)\right]\right. \\
& \left.\left.+i \epsilon^{\mu \rho \nu \eta}\left[\bar{\psi}_{f}(-z / 2) \gamma_{\eta} \psi_{f}(z / 2)+(z \rightarrow-z)\right]\right]\right\} .
\end{aligned}
$$

In contrast to the standard electromagnetic DVCS process, see Eq. (90), we end up with two additional terms. Namely, the presence of the axial part $\gamma_{5} c_{A}^{f}$ of the $V$ - $A$ interaction gives rise to a vector current symmetric in the Lorentz indices $\mu, \nu$ and to an axial vector current antisymmetric in $\mu, \nu$. The string operators here are accompanied either with $Q_{f} c_{V}^{f}$ or $Q_{f} c_{A}^{f}$ rather than $Q_{f}^{2}$. Also the denominator of Eq. (249) carries an extra factor of 2 due to the nature of the vertex $q q Z^{0}$.

Accordingly, to obtain the twist-2 part of Eq. (249) we need, in addition to contracted string operators given by Eq. (94), two extra operators. One can conveniently write all four contracted vector and axial vector string operators under the same footing as

$$
\begin{aligned}
\mathcal{O}^{f \pm}(z) & \equiv\left[\bar{\psi}_{f}(-z / 2) \not z \psi_{f}(z / 2) \pm(z \rightarrow-z)\right] \\
\mathcal{O}_{5}^{f \pm}(z) & \equiv\left[\bar{\psi}_{f}(-z / 2) \not \gamma_{5} \psi_{f}(z / 2) \pm(z \rightarrow-z)\right] .
\end{aligned}
$$

They all should satisfy the harmonic condition (95). Furthermore, the parametrization of the nonforward matrix elements of these operators is performed by means of 
the relevant nonperturbative functions, namely, the off-forward parton distributions. A straightforward generalization of the parametrization (96) from Section IV.2 can be performed by introducing a new set of GPDs, i.e. the minus OFPDs. Then

$$
\begin{aligned}
\left\langle N\left(p_{2}, s_{2}\right)\left|\mathcal{O}^{f \pm}(z)\right| N\left(p_{1}, s_{1}\right)\right\rangle_{z^{2}=0}= & \bar{u}\left(p_{2}, s_{2}\right) \not u\left(p_{1}, s_{1}\right) \\
& \times \int_{-1}^{1} d x e^{i x(p \cdot z)} H_{f}^{ \pm}(x, \xi, t) \\
& +\bar{u}\left(p_{2}, s_{2}\right) \frac{(\not p \gamma-\not \not z)}{4 M} u\left(p_{1}, s_{1}\right) \\
& \times \int_{-1}^{1} d x e^{i x(p \cdot z)} E_{f}^{ \pm}(x, \xi, t), \\
\left\langle N\left(p_{2}, s_{2}\right)\left|\mathcal{O}_{5}^{f \pm}(z)\right| N\left(p_{1}, s_{1}\right)\right\rangle_{z^{2}=0}= & \bar{u}\left(p_{2}, s_{2}\right) \not z \gamma_{5} u\left(p_{1}, s_{1}\right) \\
& \times \int_{-1}^{1} d x e^{i x(p \cdot z)} \tilde{H}_{f}^{\mp}(x, \xi, t) \\
& -\bar{u}\left(p_{2}, s_{2}\right) \frac{(r \cdot z)}{2 M} \gamma_{5} u\left(p_{1}, s_{1}\right) \\
& \times \int_{-1}^{1} d x e^{i x(p \cdot z)} \tilde{E}_{f}^{\mp}(x, \xi, t) .
\end{aligned}
$$

Note that $\mathcal{O}_{5}^{f \pm}(z)$ has a superscript opposite in sign with respect to the corresponding tilde OFPD. While the standard DVCS process gives access only to the plus distributions (recall that they correspond to the sum of quark and antiquark distributions), scattering via the weak virtual boson exchange probes also the minus distributions. It can be shown that the latter correspond to the difference in quark and antiquark distributions, i.e.the valence configuration. Like the plus OFPDs, the minus distributions have also symmetry with respect to the change $x \rightarrow-x$. Let us summarize the symmetry properties in $x$ for both plus and minus OFPDs,

$$
\begin{aligned}
H_{f}^{ \pm}(x) & =\mp H_{f}^{ \pm}(-x), \\
E_{f}^{ \pm}(x) & =\mp E_{f}^{ \pm}(-x), \\
\tilde{H}_{f}^{ \pm}(x) & = \pm \tilde{H}_{f}^{ \pm}(-x), \\
\tilde{E}_{f}^{ \pm}(x) & = \pm \tilde{E}_{f}^{ \pm}(-x) .
\end{aligned}
$$

After following the steps described in Section IV.2, we arrive at the expression for the reduced weak neutral VCA in the leading-twist approximation

$$
\begin{array}{r}
\mathcal{T}_{W N t w i s t-2}^{\mu \nu}=\frac{1}{4(p \cdot q)} \sum_{f} Q_{f} \int_{-1}^{1} \frac{d x}{(x-\xi+i 0)} \\
\times\left\{c_{V}^{f} H_{f}^{+}(x, \xi, t)\left[\frac{1}{\left(p \cdot q_{2}\right)}\left(p^{\mu} q_{2}^{\nu}+p^{\nu} q_{2}^{\mu}\right)-g^{\mu \nu}\right] \bar{u}\left(p_{2}, s_{2}\right) \not q_{2} u\left(p_{1}, s_{1}\right)\right.
\end{array}
$$




$$
\begin{array}{r}
+c_{V}^{f} E_{f}^{+}(x, \xi, t)\left[\frac{1}{\left(p \cdot q_{2}\right)}\left(p^{\mu} q_{2}^{\nu}+p^{\nu} q_{2}^{\mu}\right)-g^{\mu \nu}\right] \bar{u}\left(p_{2}, s_{2}\right) \frac{\left(q_{2} \not \gamma-\not q_{2}\right)}{4 M} u\left(p_{1}, s_{1}\right) \\
-c_{V}^{f} \tilde{H}_{f}^{+}(x, \xi, t)\left[\frac{1}{\left(p \cdot q_{2}\right)} i \epsilon^{\mu \nu \rho \eta} q_{2 \rho} p_{\eta}\right] \bar{u}\left(p_{2}, s_{2}\right) q_{2} \gamma_{5} u\left(p_{1}, s_{1}\right) \\
+c_{V}^{f} \tilde{E}_{f}^{+}(x, \xi, t)\left[\frac{1}{\left(p \cdot q_{2}\right)} i \epsilon^{\mu \nu \rho \eta} q_{2 \rho} p_{\eta}\right] \frac{\left(q_{2} \cdot r\right)}{2 M} \bar{u}\left(p_{2}, s_{2}\right) \gamma_{5} u\left(p_{1}, s_{1}\right) \\
-c_{A}^{f} \tilde{H}_{f}^{-}(x, \xi, t)\left[\frac{1}{\left(p \cdot q_{2}\right)}\left(p^{\mu} q_{2}^{\nu}+p^{\nu} q_{2}^{\mu}\right)-g^{\mu \nu}\right] \bar{u}\left(p_{2}, s_{2}\right) q_{2} \gamma_{5} u\left(p_{1}, s_{1}\right) \\
+c_{A}^{f} \tilde{E}_{f}^{-}(x, \xi, t)\left[\frac{1}{\left(p \cdot q_{2}\right)}\left(p^{\mu} q_{2}^{\nu}+p^{\nu} q_{2}^{\mu}\right)-g^{\mu \nu}\right] \frac{\left(q_{2} \cdot r\right)}{2 M} \bar{u}\left(p_{2}, s_{2}\right) \gamma_{5} u\left(p_{1}, s_{1}\right) \\
+c_{A}^{f} H_{f}^{-}(x, \xi, t)\left[\frac{1}{\left(p \cdot q_{2}\right)} i \epsilon^{\mu \nu \rho \eta} q_{2 \rho} p_{\eta}\right] \bar{u}\left(p_{2}, s_{2}\right) q_{2} u\left(p_{1}, s_{1}\right) \\
\left.+c_{A}^{f} E_{f}^{-}(x, \xi, t)\left[\frac{1}{\left(p \cdot q_{2}\right)} i \epsilon^{\mu \nu \rho \eta} q_{2 \rho} p_{\eta}\right] \bar{u}\left(p_{2}, s_{2}\right) \frac{\left(\phi_{2} \gamma-\gamma q_{2}\right)}{4 M} u\left(p_{1}, s_{1}\right)\right\}
\end{array}
$$

\section{VII.2.2 Weak Charged Amplitude}

Skipping an overall factor $-|e| g / \sqrt{2}$, the expansion of the time-ordered product of two currents in the weak charged sector reads

$$
\begin{aligned}
i T\left\{J_{E M}^{\mu}(z / 2) J_{W C}^{\nu}(-z / 2)\right\}= & -\frac{z_{\rho}}{4 \pi^{2} z^{4}} \sum_{f, f^{\prime}}\left\{Q_{f^{\prime}} \bar{\psi}_{f^{\prime}}(z / 2) \gamma^{\mu} \gamma^{\rho} \gamma^{\nu}\left(1-\gamma_{5}\right) \psi_{f}(-z / 2)\right. \\
& \left.-Q_{f} \bar{\psi}_{f}(-z / 2) \gamma^{\nu}\left(1-\gamma_{5}\right) \gamma^{\rho} \gamma^{\mu} \psi_{f^{\prime}}(z / 2)\right\} \\
= & \frac{z_{\rho}}{4 \pi^{2} z^{4}} \sum_{f, f^{\prime}}\left\{s ^ { \mu \rho \nu \eta } \left[Q_{f} \bar{\psi}_{f}(-z / 2) \gamma_{\eta} \psi_{f^{\prime}}(z / 2)\right.\right. \\
& \left.-\left(f \leftrightarrow f^{\prime}, z \rightarrow-z\right)\right] \\
& +i \epsilon^{\mu \rho \nu \eta}\left[Q_{f} \bar{\psi}_{f}(-z / 2) \gamma_{\eta} \gamma_{5} \psi_{f^{\prime}}(z / 2)\right. \\
& \left.+\left(f \leftrightarrow f^{\prime}, z \rightarrow-z\right)\right] \\
& -s^{\mu \rho \nu \eta}\left[Q_{f} \bar{\psi}_{f}(-z / 2) \gamma_{\eta} \gamma_{5} \psi_{f^{\prime}}(z / 2)\right. \\
& \left.-\left(f \leftrightarrow f^{\prime}, z \rightarrow-z\right)\right] \\
& -i \epsilon^{\mu \rho \nu \eta}\left[Q_{f} \bar{\psi}_{f}(-z / 2) \gamma_{\eta} \gamma_{5} \psi_{f^{\prime}}(z / 2)\right. \\
& \left.\left.+\left(f \leftrightarrow f^{\prime}, z \rightarrow-z\right)\right]\right\} .
\end{aligned}
$$

Here the sum over quark flavors is subject to an extra condition, $Q_{f}-Q_{f^{\prime}}=1$ or -1 , due the fact that the weak virtual boson $W^{ \pm}$carries an electric charge \pm 1 in units of $|e|$. For that reason, the initial and final nucleons are not the same particles anymore. Hence we are dealing either with the neutron-to-proton transition via the 
exchange of $W^{+}$, or with the proton-to-neutron transition via the exchange of $W^{-}$. One notices that the vector and axial vector string operators in the expansion (254) are not diagonal in quark flavor, i.e. they are accompanied by different quark flavors as well as by different electric charges. The corresponding contracted string operators, which appear when extracting the twist-2 part of Eq. (254), can be expressed as the linear combinations,

$$
\begin{aligned}
{\left[Q_{f} \bar{\psi}_{f}(-z / 2) \not \psi_{f^{\prime}}(z / 2) \pm\left(f \leftrightarrow f^{\prime}, z \rightarrow-z\right)\right] } & =Q_{ \pm} \mathcal{O}^{f f^{\prime}+}(z)+Q_{\mp} \mathcal{O}^{f f^{\prime}-}(z) \\
{\left[Q_{f} \bar{\psi}_{f}(-z / 2) \not z \gamma_{5} \psi_{f^{\prime}}(z / 2) \pm\left(f \leftrightarrow f^{\prime}, z \rightarrow-z\right)\right] } & =Q_{ \pm} \mathcal{O}_{5}^{f f^{\prime}+}(z)+Q_{\mp} \mathcal{O}_{5}^{f f^{\prime}-}(z)
\end{aligned}
$$

of operators,

$$
\begin{aligned}
& \mathcal{O}^{f f^{\prime} \pm}(z) \equiv\left[\bar{\psi}_{f}(-z / 2) \not \psi_{f}(z / 2) \pm\left(f \leftrightarrow f^{\prime}, z \rightarrow-z\right)\right] \\
& \mathcal{O}_{5}^{f f^{\prime} \pm}(z) \equiv\left[\bar{\psi}_{f}(-z / 2) \not z \gamma_{5} \psi_{f}(z / 2) \pm\left(f \leftrightarrow f^{\prime}, z \rightarrow-z\right)\right]
\end{aligned}
$$

with the coefficients $Q_{ \pm}=\left(Q_{f} \pm Q_{f^{\prime}}\right) / 2$. The matrix elements of these newly intro-

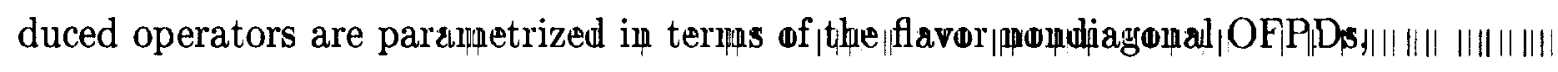

$$
\begin{aligned}
\left\langle N^{\prime}\left(p_{2}, s_{2}\right)\left|\mathcal{O}^{f f^{\prime} \pm}(z)\right| N\left(p_{1}, s_{1}\right)\right\rangle_{z^{2}=0}= & \bar{u}\left(p_{2}, s_{2}\right) \not z u\left(p_{1}, s_{1}\right) \\
& \times \int_{-1}^{1} d x e^{i x(p \cdot z)} H_{f f^{\prime}}^{ \pm}(x, \xi, t) \\
& +\bar{u}\left(p_{2}, s_{2}\right) \frac{(\not \partial \gamma-\not \gamma \not)}{4 M} u\left(p_{1}, s_{1}\right) \\
& \times \int_{-1}^{1} d x e^{i x(p \cdot z)} E_{f f^{\prime}}^{ \pm}(x, \xi, t), \\
\left\langle N^{\prime}\left(p_{2}, s_{2}\right)\left|\mathcal{O}_{5}^{f f^{\prime} \pm}(z)\right| N\left(p_{1}, s_{1}\right)\right\rangle_{z^{2}=0}= & \bar{u}\left(p_{2}, s_{2}\right) \not z \gamma_{5} u\left(p_{1}, s_{1}\right) \\
& \times \int_{-1}^{1} d x e^{i x(p \cdot z)} \tilde{H}_{f f^{\prime}}^{\mp}(x, \xi, t) \\
& -\bar{u}\left(p_{2}, s_{2}\right) \frac{(r \cdot z)}{2 M} \gamma_{5} u\left(p_{1}, s_{1}\right) \\
& \times \int_{-1}^{1} d x e^{i x(p \cdot z)} \tilde{E}_{f f^{\prime}}^{\mp}(x, \xi, t) .
\end{aligned}
$$

Finally, with the help of Eqs. (255) and (257), the leading-twist reduced weak charged VCA can be easily obtained from the result for the weak neutral case with the proper replacements. We get

$$
\mathcal{T}_{W C t w i s t-2}^{\mu \nu}=\frac{1}{4(p \cdot q)} \sum_{f, f^{\prime}} \int_{-1}^{1} \frac{d x}{(x-\xi+i 0)}
$$




$$
\begin{aligned}
& \times\left\{\left[Q_{+} H_{f f^{\prime}}^{+}(x, \xi, t)+Q_{-} H_{f f^{\prime}}^{-}(x, \xi, t)\right]\right. \\
& \times\left[\frac{1}{\left(p \cdot q_{2}\right)}\left(p^{\mu} q_{2}^{\nu}+p^{\nu} q_{2}^{\mu}\right)-g^{\mu \nu}\right] \bar{u}\left(p_{2}, s_{2}\right) q_{2} u\left(p_{1}, s_{1}\right) \\
& +\left[Q_{+} E_{f f^{\prime}}^{+}(x, \xi, t)+Q_{-} E_{f f^{\prime}}^{-}(x, \xi, t)\right] \\
& \times\left[\frac{1}{\left(p \cdot q_{2}\right)}\left(p^{\mu} q_{2}^{\nu}+p^{\nu} q_{2}^{\mu}\right)-g^{\mu \nu}\right] \bar{u}\left(p_{2}, s_{2}\right) \frac{\left(q_{2} \not-\not / q_{2}\right)}{4 M} u\left(p_{1}, s_{1}\right) \\
& -\left[Q_{+} \tilde{H}_{f f^{\prime}}^{+}(x, \xi, t)+Q_{-} \tilde{H}_{f f^{\prime}}^{-}(x, \xi, t)\right] \\
& \times\left[\frac{1}{\left(p \cdot q_{2}\right)} i \epsilon^{\mu \nu \rho \eta} q_{2 \rho} p_{\eta}\right] \bar{u}\left(p_{2}, s_{2}\right) \not q_{2} \gamma_{5} u\left(p_{1}, s_{1}\right) \\
& +\left[Q_{+} \tilde{E}_{f f^{\prime}}^{+}(x, \xi, t)+Q_{-} \tilde{E}_{f f^{\prime}}^{-}(x, \xi, t)\right] \\
& \times\left[\frac{1}{\left(p \cdot q_{2}\right)} i \epsilon^{\mu \nu \rho \eta} q_{2 \rho} p_{\eta}\right] \frac{\left(q_{2} \cdot r\right)}{2 M} \bar{u}\left(p_{2}, s_{2}\right) \gamma_{5} u\left(p_{1}, s_{1}\right) \\
& -\left[Q_{-} \tilde{H}_{f f^{\prime}}^{+}(x, \xi, t)+Q_{+} \tilde{H}_{f f^{\prime}}^{-}(x, \xi, t)\right] \\
& \times\left[\frac{1}{\left(p \cdot q_{2}\right)}\left(p^{\mu} q_{2}^{\nu}+p^{\nu} q_{2}^{\mu}\right)-g^{\mu \nu}\right] \bar{u}\left(p_{2}, s_{2}\right) \not_{2} \gamma_{5} u\left(p_{1}, s_{1}\right) \\
& +\left[Q_{-} \tilde{E}_{f f^{\prime}}^{+}(x, \xi, t)+Q_{+} \tilde{E}_{f f^{\prime}}^{-}(x, \xi, t)\right] \\
& \times\left[\frac{1}{\left(p \cdot q_{2}\right)}\left(p^{\mu} q_{2}^{\nu}+p^{\nu} q_{2}^{\mu}\right)-g^{\mu \nu}\right] \frac{\left(q_{2} \cdot r\right)}{2 M} \bar{u}\left(p_{2}, s_{2}\right) \gamma_{5} u\left(p_{1}, s_{1}\right) \\
& +\left[Q_{-} H_{f f^{\prime}}^{+}(x, \xi, t)+Q_{+} H_{f f^{\prime}}^{-}(x, \xi, t)\right] \\
& \times\left[\frac{1}{\left(p \cdot q_{2}\right)} i \epsilon^{\mu \nu \rho \eta} q_{2 \rho} p_{\eta}\right] \bar{u}\left(p_{2}, s_{2}\right) \not_{2} u\left(p_{1}, s_{1}\right) \\
& +\left[Q_{-} E_{f f^{\prime}}^{+}(x, \xi, t)+Q_{+} E_{f f^{\prime}}^{-}(x, \xi, t)\right] \\
& \left.\times\left[\frac{1}{\left(p \cdot q_{2}\right)} i \epsilon^{\mu \nu \rho \eta} q_{2 \rho} p_{\eta}\right] \bar{u}\left(p_{2}, s_{2}\right) \frac{\left(q_{2} \gamma-\not / q_{2}\right)}{4 M} u\left(p_{1}, s_{1}\right)\right\} .
\end{aligned}
$$

\section{VII.3 WEAK DVCS PROCESSES}

In the following section, we discuss three examples of the weak DVCS processes. Their kinematics has already been analyzed in detail in Section IV.3. The only difference is that the incoming virtual photon $\gamma^{*}$ of the standard DVCS process is now being substituted by the virtual weak boson $B$. We use the OFPD model from Section IV.4. Note that by neglecting the sea quark contribution, the plus OFPDs become equal to the minus ones, and hence we write $H_{f}^{+}=H_{f}^{-} \equiv H_{f}^{v a l}, \tilde{H}_{f}^{+}=\tilde{H}_{f}^{-} \equiv \tilde{H}_{f}^{\text {val }}$, $E_{f}^{+}=E_{f}^{-} \equiv E_{f}^{v a l}$ and $\tilde{E}_{f}^{+}=\tilde{E}_{f}^{-} \equiv \tilde{E}_{f}^{v a l}$. For the weak neutral current scattering process, we discuss neutrino-nucleon and electron-nucleon scattering. We present 


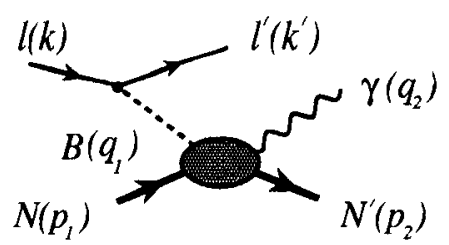

a

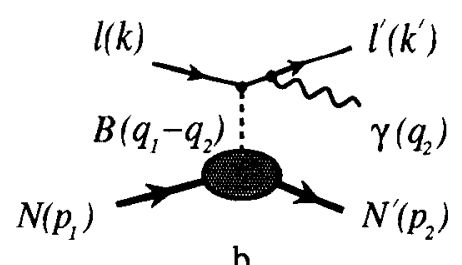

b

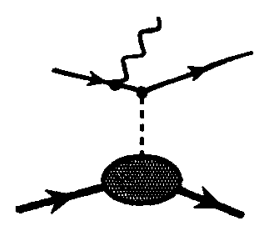

C

FIG. 39: DVCS (a) and Bethe-Heitler (b and c) diagrams in the weak deeply virtual Compton scattering process. The incoming leptons can be either neutrinos or electrons.

the calculation by taking an unpolarized proton as a target nucleon. In the case of an unpolarized neutron target, one can simply use the isospin (i.e. the charge) symmetry to express the neutron GPDs in terms of the proton ones. In the weak charged current interaction sector, we consider neutrino scattering off a neutron via the exchange of a $W^{+}$boson producing a proton in the final state. All three examples are illustrated in one figure, see Fig. 39. As in Section IV.5, the relevant cross sections are plotted against the scattering angle $\theta_{B \gamma}$ between the directions of the incoming weak virtual boson and the outgoing real photon for one kinematical point with $Q_{1}^{2}=2.5 \mathrm{GeV}^{2}, x_{B}=0.35$ and $\varphi=0$, however, for two different lepton beam energies. We take $\omega=5 \mathrm{GeV}$ and $20 \mathrm{GeV}$ rather than $\omega=5.75 \mathrm{GeV}$ and $11 \mathrm{GeV}$. Similarly to the standard DVCS process, the Bethe-Heitler cross sections are presented on a logarithmic scale as well as the plots with both contributions. In addition, for given kinematical regions, $0.28 \leq x_{B} \leq 0.3$ with $\omega=5 \mathrm{GeV}$ and $0.26 \leq x_{B} \leq 0.28$ with $\omega=20 \mathrm{GeV}$, we estimate the orders of magnitude for the total cross sections, coming purely from the DVCS diagrams and compare them with the Compton contribution to the standard electromagnetic DVCS process. They are listed in Table IV at the end of this chapter.

\section{VII.3.1 Weak Neutral Neutrino-Proton Scattering}

The photon cannot be emitted by the neutrino, and thus in neutrino scattering from a proton via the exchange of $Z^{0}$, we only measure the Compton part. The T-matrix of the process is given solely by the DVCS diagram, i.e. the diagram (a) in Fig. 39,

$$
i \mathrm{~T}_{\nu p}=\bar{u}\left(k^{\prime}\right)\left(\frac{-i g}{\cos \theta_{W}}\right) \gamma^{\lambda}\left(\frac{c_{V}^{\nu}-\gamma_{5} c_{A}^{\nu}}{2}\right) u(k)\left[\frac{-i\left(g_{\nu \lambda}-q_{1 \nu} q_{1 \lambda} / M_{Z^{0}}^{2}\right)}{q_{1}^{2}-M_{Z^{0}}^{2}}\right]
$$




$$
\times\left(\frac{-|e| g}{\cos \theta_{W}}\right) \epsilon_{\mu}^{*}\left(q_{2}\right)\left(-i \mathcal{T}_{W N}^{\mu \nu}\right)
$$

with $\mathcal{T}_{W N}^{\mu \nu}$ computed in the handbag approximation from the diagrams shown in Fig. 38. The vector and axial vector couplings of the vertex $\nu \nu Z^{0}$ are $c_{V}^{\nu}=c_{A}^{\nu}=1 / 2$. Furthermore, since $M_{Z^{\circ}}^{2} \gg Q_{1}^{2}$ and $\cos \theta_{W} \equiv M_{W} / M_{Z^{\circ}}$ (recall that the Fermi constant is $G_{F} \equiv g^{2} / 4 \sqrt{2} M_{W}^{2}$ ), the amplitude turns into

$$
\mathrm{T}_{\nu p}=\sqrt{2}|e| G_{F} \bar{u}\left(k^{\prime}\right) \gamma_{\nu}\left(1-\gamma_{5}\right) u(k) \epsilon_{\mu}^{*}\left(q_{2}\right) \mathcal{T}_{W N}^{\mu \nu}
$$

Its spin-averaged square then reads

$$
\overline{\left|\mathrm{T}_{\nu p}\right|^{2}}=8 \pi \alpha G_{F}^{2} L_{\nu \beta}^{(\nu)} H_{W N}^{\nu \beta}
$$

The neutrino tensor is simply

$$
L_{\nu \beta}^{(\nu)}=8\left[k_{\nu} k_{\beta}^{\prime}+k_{\beta} k_{\nu}^{\prime}-g_{\nu \beta}\left(k \cdot k^{\prime}\right)+i \epsilon_{\nu \beta \sigma \tau} k^{\sigma} k^{\prime \tau}\right]
$$

On the other hand, the weak neutral hadron tensor has a rather complicated expression. Nevertheless, in the DVCS kinematics, it reduces to

$$
\begin{aligned}
H_{W N}^{\nu \beta}= & -\frac{1}{2} \mathcal{T}_{W N}^{\mu \nu}\left(\mathcal{T}_{\mu W N}^{\beta}\right)^{*} \\
= & -\frac{1}{4}\left\{\left[\left(1-\xi^{2}\right)\left(\left|\mathcal{H}_{W N}^{+}\right|^{2}+\left|\mathcal{H}_{W N}^{-}\right|^{2}+\left|\tilde{\mathcal{H}}_{W N}^{+}\right|^{2}+\left|\tilde{\mathcal{H}}_{W N}^{-}\right|^{2}\right)\right.\right. \\
& -\left(\xi^{2}+\frac{t}{4 M^{2}}\right)\left(\left|\mathcal{E}_{W N}^{+}\right|^{2}+\left|\mathcal{E}_{W N}^{-}\right|^{2}\right)-\xi^{2} \frac{t}{4 M^{2}}\left(\left|\tilde{\mathcal{E}}_{W N}^{+}\right|^{2}+\left|\tilde{\mathcal{E}}_{W N}^{-}\right|^{2}\right) \\
& \left.-2 \xi^{2} \Re\left(\mathcal{H}_{W N}^{+*} \mathcal{E}_{W N}^{+}+\mathcal{H}_{W N}^{-*} \mathcal{E}_{W N}^{-}+\tilde{\mathcal{H}}_{W N}^{+*} \tilde{\mathcal{E}}_{W N}^{+}+\tilde{\mathcal{H}}_{W N}^{-*} \tilde{\mathcal{E}}_{W N}^{-}\right)\right] \\
& \times\left[g^{\nu \beta}-\frac{1}{\left(p \cdot q_{2}\right)}\left(p^{\nu} q_{2}^{\beta}+p^{\nu} q_{2}^{\beta}\right)+\frac{M^{2}}{\left(p \cdot q_{2}\right)^{2}}\left(1-\frac{t}{4 M^{2}}\right) q_{2}^{\nu} q_{2}^{\beta}\right] \\
& +2\left[\left(1-\xi^{2}\right) \Re\left(\mathcal{H}_{W N}^{+*} \mathcal{H}_{W N}^{-}+\tilde{\mathcal{H}}_{W N}^{+*} \tilde{\mathcal{H}}_{W N}^{-}\right)\right. \\
& -\left(\xi^{2}+\frac{t}{4 M^{2}}\right) \Re\left(\mathcal{E}_{W N}^{+*} \mathcal{E}_{W N}^{-}\right)-\xi^{2} \frac{t}{4 M^{2}} \Re\left(\tilde{\mathcal{E}}_{W N}^{+*} \tilde{\mathcal{E}}_{W N}^{-}\right) \\
& \left.-\xi^{2} \Re\left(\mathcal{H}_{W N}^{+*} \mathcal{E}_{W N}^{-}+\mathcal{E}_{W N}^{+*} \mathcal{H}_{W N}^{-}+\tilde{\mathcal{H}}_{W N}^{+*} \tilde{\mathcal{E}}_{W N}^{-}+\tilde{\mathcal{E}}_{W N}^{+*} \tilde{\mathcal{H}}_{W N}^{-}\right)\right] \\
& \left.\times \frac{1}{\left(p \cdot q_{2}\right)} i \epsilon^{\nu \beta \delta \lambda} p_{\delta} q_{2 \lambda}\right\}
\end{aligned}
$$

where the integrals of OFPDs are conveniently defined as

$$
\begin{aligned}
\mathcal{H}_{W N}^{+(-)}(\xi, t) & \equiv \sum_{f} Q_{f} c_{V(A)}^{f} \int_{-1}^{1} \frac{d x}{(x-\xi+i 0)} H_{f}^{+(-)}(x, \xi, t) \\
\mathcal{E}_{W N}^{+(-)}(\xi, t) & \equiv \sum_{f} Q_{f} c_{V(A)}^{f} \int_{-1}^{1} \frac{d x}{(x-\xi+i 0)} E_{f}^{+(-)}(x, \xi, t)
\end{aligned}
$$




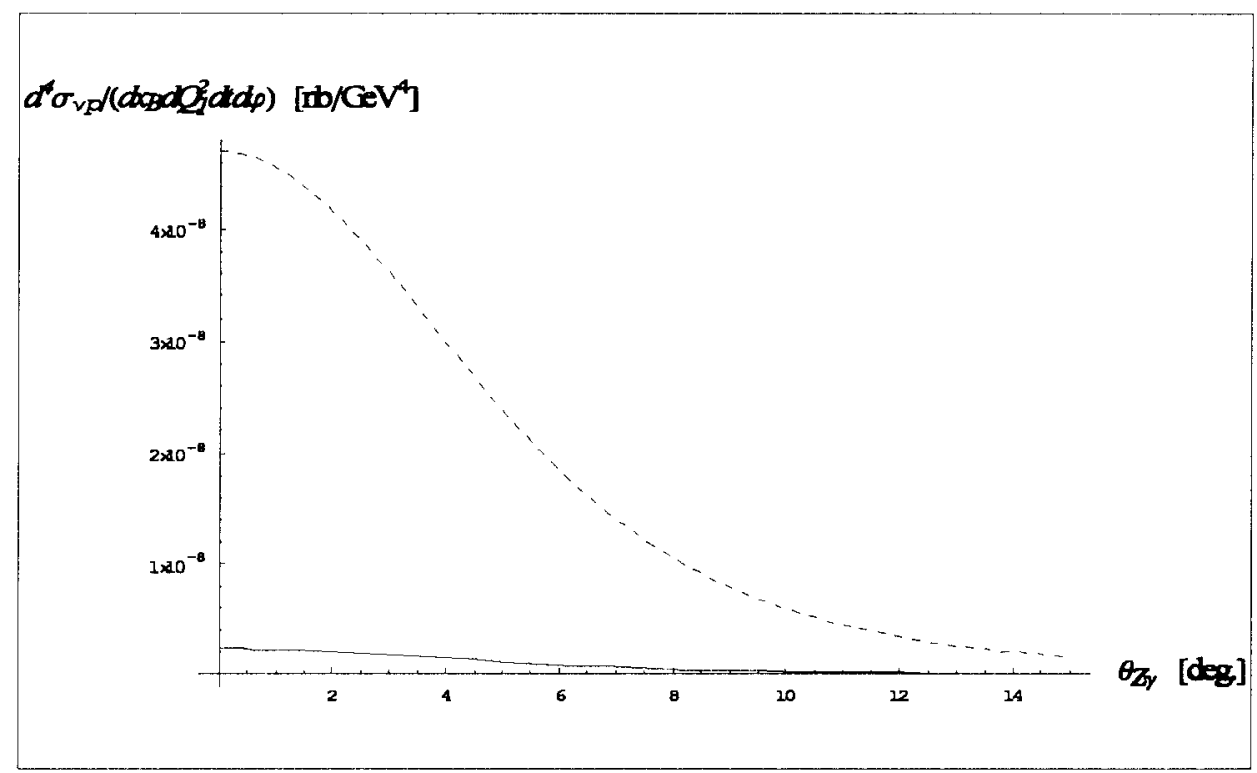

FIG. 40: Weak neutral DVCS cross section $\sigma_{\nu p}$ plotted as a function of the angle $\theta_{\boldsymbol{B} \gamma}$ between the incoming weak virtual boson and outgoing real photon in the target rest frame for $Q_{1}^{2}=2.5 \mathrm{GeV}^{2}$ and $x_{B}=0.35$ with $\omega=5 \mathrm{GeV}$ (solid line) and $\omega=20 \mathrm{GeV}$ (dashed line) neutrino beam.

$$
\begin{aligned}
\tilde{\mathcal{H}}_{W N}^{+(-)}(\xi, t) & \equiv \sum_{f} Q_{f} c_{V(A)}^{f} \int_{-1}^{1} \frac{d x}{(x-\xi+i 0)} \tilde{H}_{f}^{+(-)}(x, \xi, t), \\
\tilde{\mathcal{E}}_{W N}^{+(-)}(\xi, t) & \equiv \sum_{f} Q_{f} c_{V(A)}^{f} \int_{-1}^{1} \frac{d x}{(x-\xi+i 0)} \tilde{E}_{f}^{+(-)}(x, \xi, t) .
\end{aligned}
$$

After the substitution of Eq. (261) into Eq. (119), the unpolarized differential cross section for the weak neutral DVCS process on a proton target using the neutrino beam takes the following form

$$
\frac{d^{4} \sigma_{\nu p}}{d x_{B} d Q_{1}^{2} d t d \varphi}=\frac{1}{(2 \pi)^{3}} \frac{\alpha G_{F}^{2}}{16} \frac{1+x_{B}(M / \omega)}{M^{2} \omega^{2}[2+(M / \omega)] x_{B}\left[y+2 x_{B}(M / \omega)\right]^{2}} L_{\nu \beta}^{(\nu)} H_{W N}^{\nu \beta},
$$

The cross section is illustrated in Fig. 40.

\section{VII.3.2 Weak Neutral Electron-Proton Scattering}

In the electron scattering, one has to include both the Compton and the Bethe-Heitler contributions, $\mathrm{T}_{e p}=\mathrm{T}_{C e p}+\mathrm{T}_{B H e p}$. The T-matrix for the Compton part can be easily obtained from Eq. (259) by replacing the neutrinos with the electrons,

$$
\mathrm{T}_{C e p}=2 \sqrt{2}|e| G_{F} \bar{u}\left(k^{\prime}\right) \gamma_{\nu}\left(c_{V}^{e}-\gamma_{5} c_{A}^{e}\right) u(k) \epsilon_{\mu}^{*}\left(q_{2}\right) \mathcal{T}_{W N}^{\mu \nu}
$$




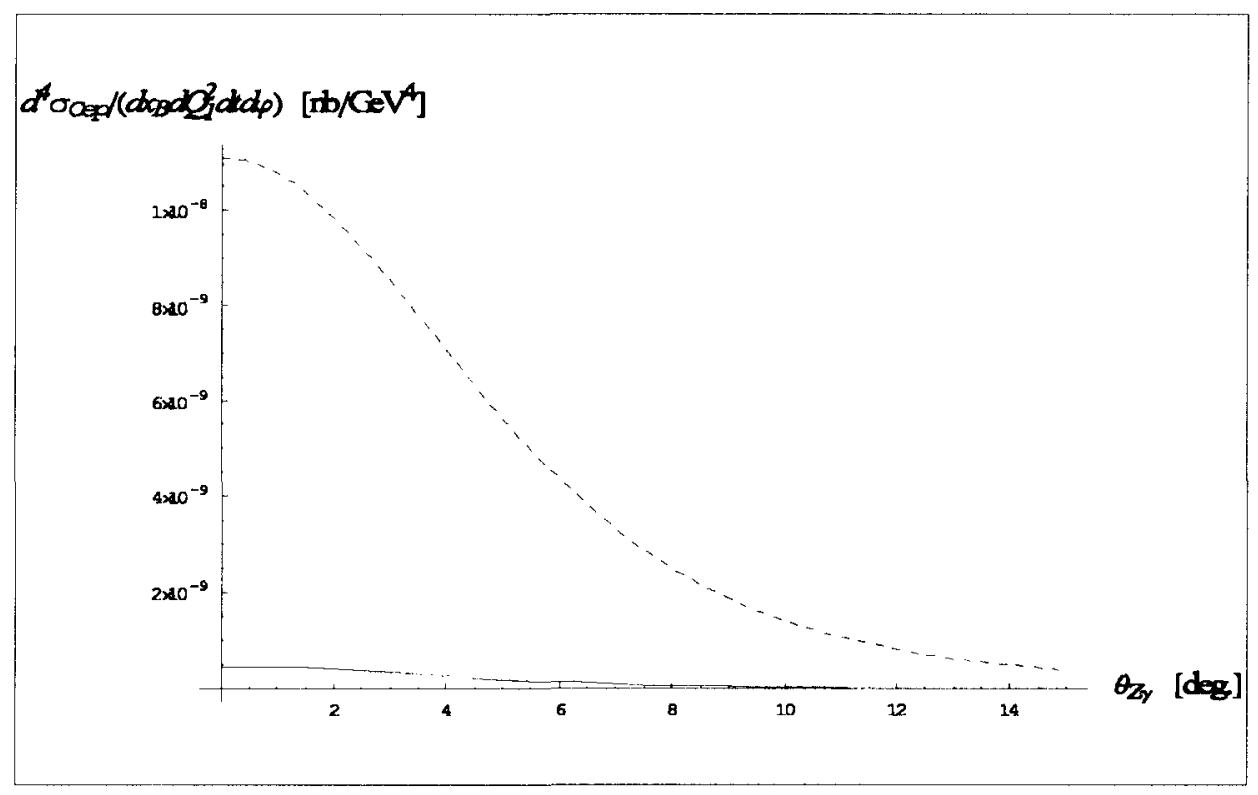

FIG. 41: Compton cross section $\sigma_{C e p}$ for the weak neutral DVCS process plotted as a function of the angle $\theta_{B \gamma}$ between the incoming weak virtual boson and outgoing real photon in the target rest frame for $Q_{1}^{2}=2.5 \mathrm{GeV}^{2}$ and $x_{B}=0.35$ with $\omega=5 \mathrm{GeV}$ (solid line) and $\omega=20 \mathrm{GeV}$ (dashed line) electron beam.

The couplings are now equal to $c_{V}^{e}=-1 / 2+2 \sin ^{2} \theta_{W}$ and $c_{A}^{e}=-1 / 2$. Note that the hadron tensor is the same as in the neutrino case. Due to averaging over the spin of incoming electrons, the electron tensor has an extra factor of $1 / 2$,

$$
L_{\nu \beta}^{(e)}=2\left\{\left[\left(c_{V}^{e}\right)^{2}+\left(c_{A}^{e}\right)^{2}\right]\left[k_{\nu} k_{\beta}^{\prime}+k_{\beta} k_{\nu}^{\prime}-g_{\nu \beta}\left(k \cdot k^{\prime}\right)\right]-2 c_{V}^{e} c_{A}^{e} i \epsilon_{\nu \beta \sigma \tau} k^{\sigma} k^{\prime \tau}\right\} .
$$

The unpolarized Compton differential cross section for the weak neutral DVCS process with a proton target and an electron beam reads

$$
\frac{d^{4} \sigma_{C e p}}{d x_{B} d Q_{1}^{2} d t d \varphi}=\frac{1}{(2 \pi)^{3}} \frac{\alpha G_{F}^{2}}{4} \frac{1+x_{B}(M / \omega)}{M^{2} \omega^{2}[2+(M / \omega)] x_{B}\left[y+2 x_{B}(M / \omega)\right]^{2}} L_{\nu \beta}^{(e)} H_{W N}^{\nu \beta},
$$

and it is plotted in Fig. 41.

Next we investigate the Bethe-Heitler contribution to the process. Since incoming and scattered leptons are electrons, we have to include both Bethe-Heitler diagrams, see the diagrams (b) and (c) in Fig. 39. The Bethe-Heitler amplitude can be immediately written down with the help of the expression (140) along with the following modifications: 
- The vertex eer is replaced by the vertex ee $Z^{0}$, $i|e| \gamma^{\nu} \rightarrow-i g \gamma^{\nu}\left(c_{V}^{e}-\gamma_{5} c_{A}^{e}\right) / 2 \cos \theta_{W}$

- The photon propagator is replaced by the weak boson propagator, $-i g_{\nu \lambda} /\left(q_{1}-q_{2}\right)^{2} \rightarrow i g_{\nu \lambda} / M_{Z^{0}}^{2}$.

- The coupling $\gamma p$ is replaced by the coupling $Z^{0} p,-i|e| \rightarrow-i g / \cos \theta_{W}$.

- The proton electromagnetic transition current is replaced by the proton weak neutral transition current, $J_{E M}^{\lambda}(0) \rightarrow J_{N C}^{\lambda}(0)$.

Hence

$$
\begin{aligned}
\mathrm{T}_{B H e p}= & 2 \sqrt{2}|e| G_{F} \epsilon_{\mu}^{*}\left(q_{2}\right) \bar{u}\left(k^{\prime}\right)\left[\frac{\gamma^{\mu}\left(\not k^{\prime}+\not q_{2}\right) \gamma^{\nu}\left(c_{V}^{e}-\gamma_{5} c_{A}^{e}\right)}{\left(k^{\prime}+q_{2}\right)^{2}}\right. \\
& \left.+\frac{\gamma^{\nu}\left(c_{V}^{e}-\gamma_{5} c_{A}^{e}\right)\left(\not k-\not q_{2}\right) \gamma^{\mu}}{\left(k-q_{2}\right)^{2}}\right] u(k)\left\langle p\left(p_{2}, s_{2}\right)\left|J_{\nu}^{N C}(0)\right| p\left(p_{1}, s_{1}\right)\right\rangle,
\end{aligned}
$$

where the transition current,

$$
\begin{aligned}
J_{\nu}^{N C}(0) & =\frac{1}{2}\left[V_{\nu}^{N C}(0)-A_{\nu}^{N C}(0)\right] \\
& =\frac{1}{2} \sum_{f}\left[c_{V}^{f} \bar{\psi}_{f}(0) \gamma_{\nu} \psi_{f}(0)-c_{A}^{f} \bar{\psi}_{f}(0) \gamma_{\nu} \gamma_{5} \psi_{f}(0)\right]
\end{aligned}
$$

has both the vector $V_{\nu}^{N C}$ and axial vector $A_{\nu}^{N C}$ contributions. Their proton matrix elements are parametrized in terms of the four form factors [42],

$$
\begin{aligned}
\left\langle p\left(p_{2}, s_{2}\right)\left|V_{\nu}^{N C}(0)\right| p\left(p_{1}, s_{1}\right)\right\rangle & =\bar{u}\left(p_{2}, s_{2}\right)\left[F_{1}^{N C}(t) \gamma_{\nu}-F_{2}^{N C}(t) \frac{i \sigma_{\nu \lambda} r^{\lambda}}{2 M}\right] u\left(p_{1}, s_{1}\right), \\
\left\langle p\left(p_{2}, s_{2}\right)\left|A_{\nu}^{N C}(0)\right| p\left(p_{1}, s_{1}\right)\right\rangle & =\bar{u}\left(p_{2}, s_{2}\right)\left[G_{A}^{N C}(t) \gamma_{\nu} \gamma_{5}-G_{P}^{N C}(t) \frac{\gamma_{5} r_{\nu}}{2 M}\right] u\left(p_{1}, s_{1}\right) .
\end{aligned}
$$

Each of the vector form factors can be further expressed as a linear combination of flavor triplet, octet and singlet form factors (they correspond to the proton matrix elements of the appropriate linear combinations of quark vector currents, $\left(\bar{u} \gamma_{\nu} u-\bar{d} \gamma_{\nu} d\right) / 2,\left(\bar{u} \gamma_{\nu} u+\bar{d} \gamma_{\nu} d-2 \bar{s} \gamma_{\nu} s\right)$ and $\left(\bar{u} \gamma_{\nu} u+\bar{d} \gamma_{\nu} d+\bar{s} \gamma_{\nu} s\right)$, respectively),

$$
F_{1(2)}^{N C}(t)=\left(1-2 \sin ^{2} \theta_{W}\right)\left[F_{1(2)}^{3}(t)+\frac{1}{6} F_{1(2)}^{8}(t)\right]-\frac{1}{6} F_{1(2)}^{0}(t) .
$$

Moreover. the axial and pseudoscalar form factors are expressed as the difference between the isovector and strangeness form factors (they correspond to the proton 
matrix elements of current combinations, $\left(\bar{u} \gamma_{\nu} \gamma_{5} u-\bar{d} \gamma_{\nu} \gamma_{5} d\right) / 2$ and $\left(\bar{s} \gamma_{\nu} \gamma_{5} s\right)$, respectively),

$$
G_{A(P)}^{N C}(t)=G_{A(P)}^{3}(t)-\frac{1}{2} G_{A(P)}^{s}(t)
$$

If the sea quarks are neglected, they all simplify to

$$
\begin{aligned}
F_{1(2)}^{3} & =\frac{1}{2}\left[F_{1(2) u}-F_{1(2) d}\right], \\
F_{1(2)}^{8}=F_{1(2)}^{0} & =\left[F_{1(2) u}+F_{1(2) d}\right]
\end{aligned}
$$

and

$$
\begin{aligned}
G_{A}^{N C}(t) & =\frac{g_{A}(t=0)}{2}\left(1-\frac{t}{m_{A}^{2}}\right)^{-2}, \\
G_{P}^{N C}(t) & =\frac{G_{A}^{N C}(t)}{2} \frac{4 M^{2}}{m_{\pi}^{2}-t} .
\end{aligned}
$$

For the unpolarized Bethe-Heitler differential cross section we have

$$
\frac{d^{4} \sigma_{B H e p}}{d x_{B} d Q_{1}^{2} d t d \varphi}=\frac{1}{(2 \pi)^{3}} \frac{\alpha G_{F}^{2}}{4} \frac{1+x_{B}(M / \omega)}{M^{2} \omega^{2}[2+(M / \omega)] x_{B}\left[y+2 x_{B}(M / \omega)\right]^{2}} L_{B H}^{\nu \beta} H_{\nu \beta}^{B H},
$$

where the electron tensor is

$$
\begin{aligned}
L_{B H}^{\nu \beta}= & -\frac{1}{2} \operatorname{Tr}\left\{\not k^{\prime}\left[\frac{\left(\gamma^{\mu} \not_{2}+2 k^{\prime \mu}\right) \gamma^{\nu}\left(c_{V}^{e}-\gamma_{5} c_{A}^{e}\right)}{2\left(k^{\prime} \cdot q_{2}\right)}+\frac{\gamma^{\nu}\left(c_{V}^{e}-\gamma_{5} c_{A}^{e}\right)\left(\not_{2} \gamma^{\mu}-2 k^{\mu}\right)}{2\left(k \cdot q_{2}\right)}\right]\right. \\
& \left.\times \not k\left[\frac{\gamma^{\beta}\left(c_{V}^{e}-\gamma_{5} c_{A}^{e}\right)\left(d_{2} \gamma_{\mu}+2 k_{\mu}^{\prime}\right)}{2\left(k^{\prime} \cdot q_{2}\right)}+\frac{\left(\gamma_{\mu} \not q_{2}-2 k_{\nu}\right) \gamma^{\beta}\left(c_{V}^{e}-\gamma_{5} c_{A}^{e}\right)}{2\left(k \cdot q_{2}\right)}\right]\right\},
\end{aligned}
$$

and the hadron tensor is given by

$$
H_{\nu \beta}^{B H}=\frac{1}{2} \sum_{s_{1}, s_{2}}\left\langle p\left(p_{2}, s_{2}\right)\left|J_{\nu}^{N C}(0)\right| p\left(p_{1}, s_{1}\right)\right\rangle\left\langle p\left(p_{2}, s_{2}\right)\left|J_{\beta}^{N C}(0)\right| p\left(p_{1}, s_{1}\right)\right\rangle^{*} .
$$

The angular dependence of the cross section is shown in Figs. 42 and 43 . The two poles at the locations $\phi$ and $\phi^{\prime}$ from Table III are clearly seen. In Fig. 44, both contributions are plotted together. Contrary what one might expect from the results on the standard DVCS, the Compton cross section in the weak neutral interaction sector is larger, or at least comparable, with the corresponding Bethe-Heitler cross section for the scattering angles $\theta_{Z_{\gamma}} \leq 8^{0}$, which gives the region $0.15 \mathrm{GeV}^{2} \leq-t \leq$ $0.572 \mathrm{GeV}^{2}$ in the invariant momentum transfer, see Fig. 15, and hence the ratio $-t / Q_{1}^{2} \leq 0.23$. 
TABLE III: Polar angles $\phi$ and $\phi^{\prime}$ of the incoming and scattered leptons, respectively, in the target rest frame for $Q_{1}^{2}=2.5 \mathrm{GeV}^{2}$ and $x_{B}=0.35$ with two different lepton beam energies $\omega$.

\begin{tabular}{lcc}
\hline \hline$\omega[\mathrm{GeV}]$ & $\phi[\mathrm{deg}]$. & $\phi^{\prime}[\mathrm{deg}]$. \\
\hline 5 & 10.3 & 47.9 \\
20 & 20.2 & 25.3 \\
\hline \hline
\end{tabular}

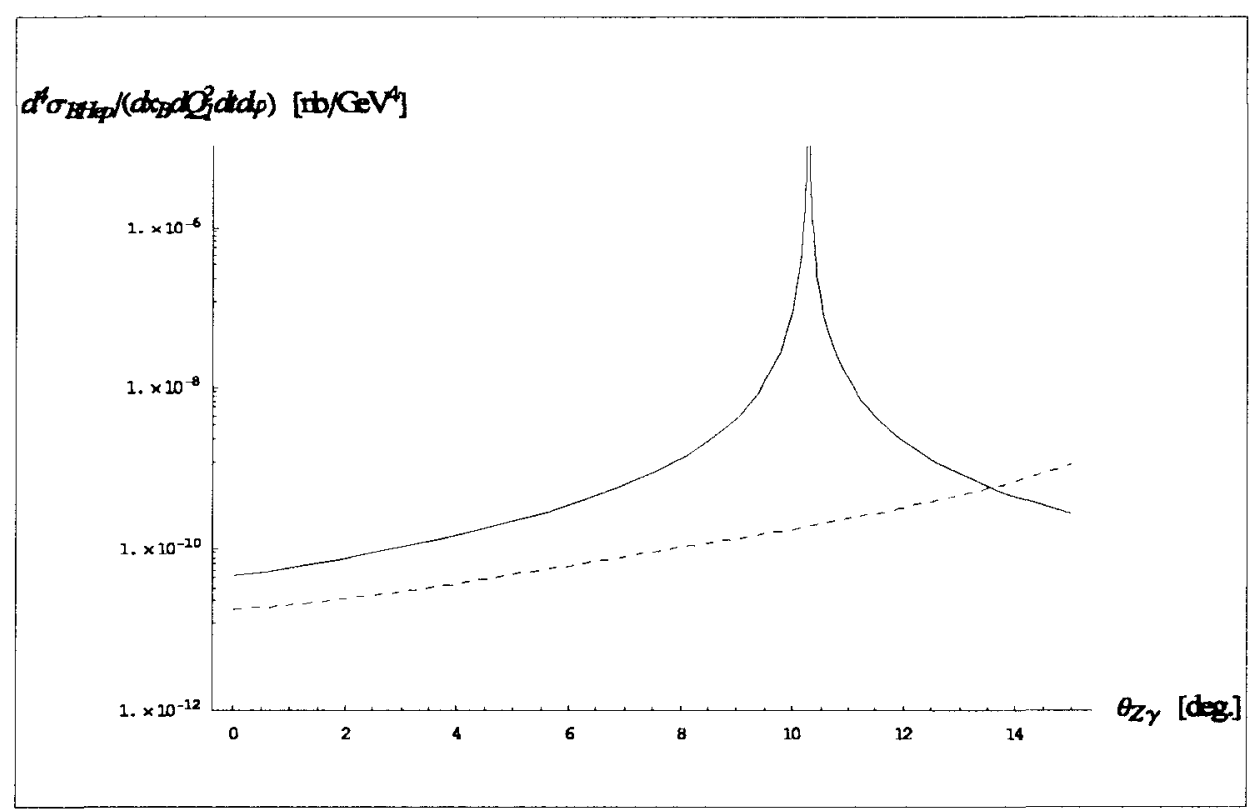

FIG. 42: Bethe-Heitler cross section $\sigma_{B H e p}$ for the weak neutral DVCS process plotted as a function of the angle $\theta_{B \gamma}$ between the incoming weak virtual boson and outgoing real photon in the target rest frame for $Q_{1}^{2}=2.5 \mathrm{GeV}^{2}$ and $x_{B}=0.35$ with $\omega=5 \mathrm{GeV}$ (solid line) and $\omega=20 \mathrm{GeV}$ (dashed line) electron beam. 


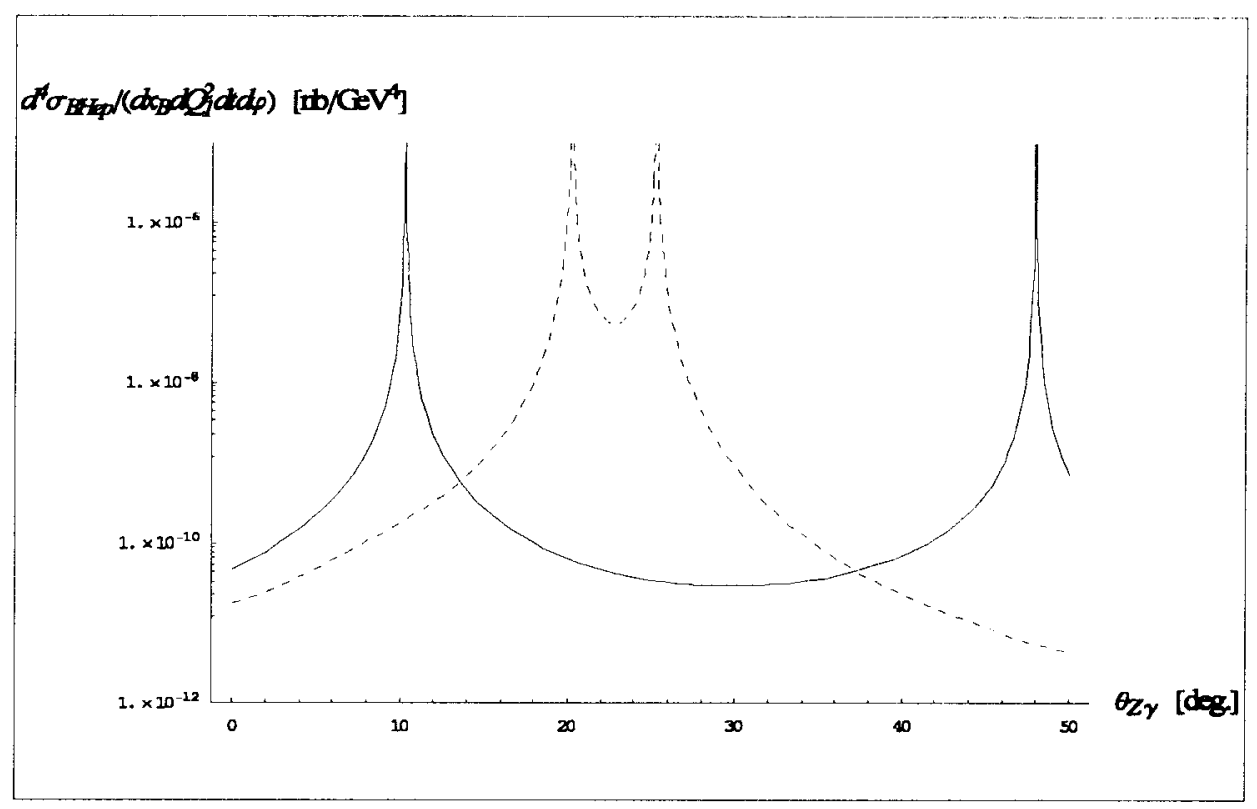

FIG. 43: Bethe-Heitler cross section $\sigma_{B H e p}$ for the weak neutral DVCS process plotted as a function of the angle $\theta_{B \gamma}$ between the incoming weak virtual boson and outgoing real photon in the target rest frame for $Q_{1}^{2}=2.5 \mathrm{GeV}^{2}$ and $x_{B}=0.35$ with $\omega=5 \mathrm{GeV}$ (solid line) and $\omega=20 \mathrm{GeV}$ (dashed line) electron beam.

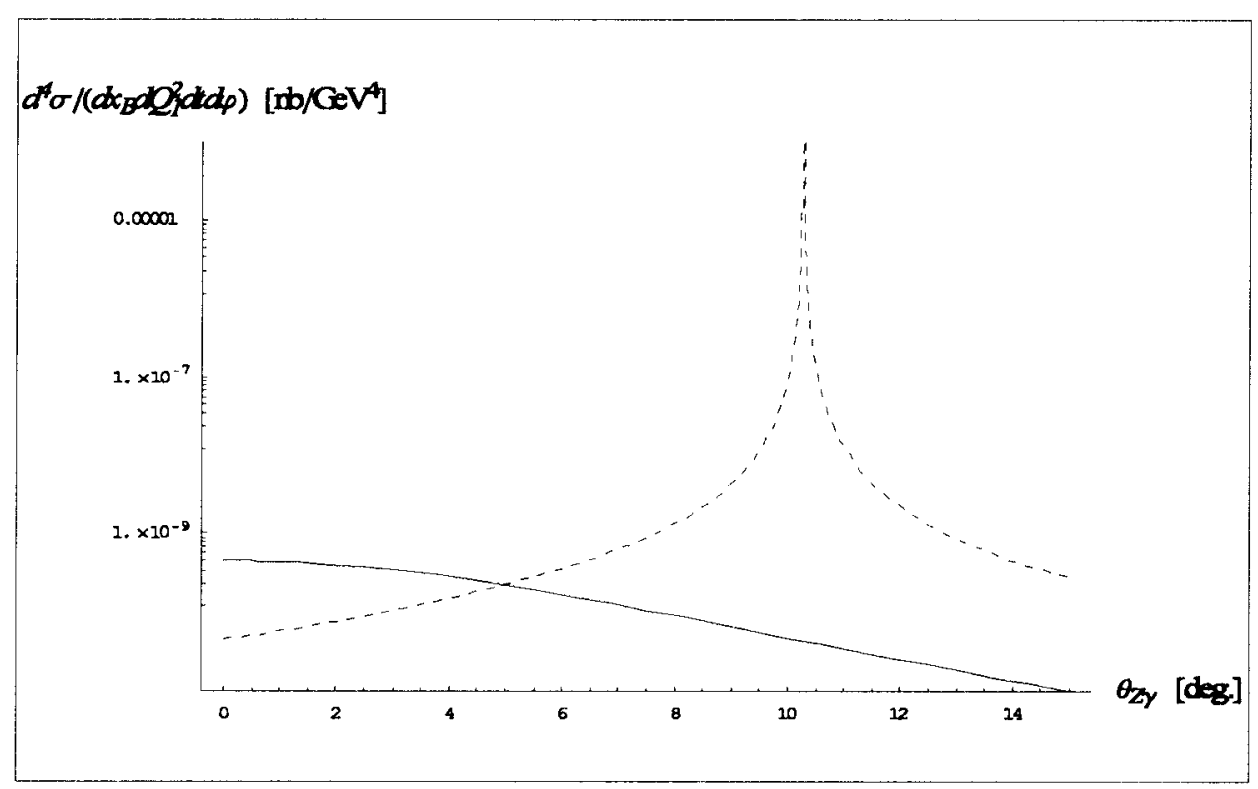

FIG. 44: Compton cross section $\sigma_{C e p}$ (solid line) and Bethe-Heitler cross section $\sigma_{B H e p}$ (dashed line) for the weak neutral DVCS process plotted as a function of the angle $\theta_{B \gamma}$ between the incoming weak virtual boson and outgoing real photon in the target rest frame for $Q_{1}^{2}=2.5 \mathrm{GeV}^{2}$ and $x_{B}=0.35$ with $\omega=5 \mathrm{GeV}$ electron beam. 


\section{VII.3.3 Weak Charged Neutrino-Neutron Scattering}

The invariant matrix element for the Compton contribution is

$$
\mathrm{T}_{C \nu n}=\sqrt{2}|e| G_{F} \bar{u}\left(k^{\prime}\right) \gamma_{\mu}\left(1-\gamma_{5}\right) u(k) \epsilon_{\mu}^{*}\left(q_{2}\right) \mathcal{T}_{W C}^{\mu \nu}
$$

where the amplitude $\mathcal{T}_{W C}^{\mu \nu}$ is, in general, given by Eq. (258). However, in our simple model the quark flavors $f$ and $f^{\prime}$ in Eqs. (258) become $d$ and $u$, respectively, and the coefficients are therefore equal to $Q_{+}=1 / 6$ and $Q_{-}=1 / 2$. In the following, we relate the flavor nondiagonal GPDs to the flavor diagonal ones. This can be achieved by using the isospin symmetry. Through the isospin symmetry, the nucleon matrix elements, $\left\langle p\left(p_{2}, s_{2}\right)\left|\mathcal{O}^{u d \pm}(z)\right| n\left(p_{1}, s_{1}\right)\right\rangle$ and $\left\langle p\left(p_{2}, s_{2}\right)\left|\mathcal{O}_{5}^{u d \pm}(z)\right| n\left(p_{1}, s_{1}\right)\right\rangle$ given by Eq. (256) that are nondiagonal in quark flavor, can be expressed in terms of the flavor diagonal nucleon matrix elements [110]. Namely,

$$
\begin{aligned}
\left\langle p\left(p_{2}, s_{2}\right)\left|\mathcal{O}^{u d \pm}(z)\right| n\left(p_{1}, s_{1}\right)\right\rangle= & \left\langle p\left(p_{2}, s_{2}\right)\left|\mathcal{O}^{u \pm}(z)\right| p\left(p_{1}, s_{1}\right)\right\rangle \\
& -\left\langle p\left(p_{2}, s_{2}\right)\left|\mathcal{O}^{d \pm}(z)\right| p\left(p_{1}, s_{1}\right)\right\rangle
\end{aligned}
$$

and similarly for $\left\langle p\left(p_{2}, s_{2}\right)\left|\mathcal{O}_{5}^{u d \pm}(z)\right| n\left(p_{1}, s_{1}\right)\right\rangle$. Accordingly, the reduced weak charged VCA assumes the form

$$
\begin{aligned}
\mathcal{T}_{W C}^{\mu \nu}= & \frac{1}{4(p \cdot q)}\left\{\left[\frac{1}{\left(p \cdot q_{2}\right)}\left(p^{\mu} q_{2}^{\nu}+p^{\nu} q_{2}^{\mu}\right)-g^{\mu \nu}\right]\right. \\
& \times\left[\mathcal{H}_{W C}^{+} \bar{u}\left(p_{2}, s_{2}\right) \phi_{2} u\left(p_{1}, s_{1}\right)+\mathcal{E}_{W C}^{+} \bar{u}\left(p_{2}, s_{2}\right) \frac{\left(q_{2} \not-\gamma q_{2}\right)}{4 M} u\left(p_{1}, s_{1}\right)\right. \\
& \left.-\tilde{\mathcal{H}}_{W C}^{-} \bar{u}\left(p_{2}, s_{2}\right) \not q_{2} \gamma_{5} u\left(p_{1}, s_{1}\right)+\tilde{\mathcal{E}}_{W C}^{-} \frac{\left(q_{2} \cdot r\right)}{2 M} \bar{u}\left(p_{2}, s_{2}\right) \gamma_{5} u\left(p_{1}, s_{1}\right)\right] \\
& -\left[\frac{1}{\left(p \cdot q_{2}\right)} i \epsilon^{\mu \nu \rho \eta} q_{2 \rho} p_{\eta}\right] \\
& \times\left[\tilde{\mathcal{H}}_{W C}^{+} \bar{u}\left(p_{2}, s_{2}\right) \not_{2} \gamma_{5} u\left(p_{1}, s_{1}\right)-\tilde{\mathcal{E}}_{W C}^{+} \frac{\left(q_{2} \cdot r\right)}{2 M} \bar{u}\left(p_{2}, s_{2}\right) \gamma_{5} u\left(p_{1}, s_{1}\right)\right. \\
& \left.\left.-\mathcal{H}_{W C}^{-} \bar{u}\left(p_{2}, s_{2}\right) \not q_{2} u\left(p_{1}, s_{1}\right)-\mathcal{E}_{W C}^{-} \bar{u}\left(p_{2}, s_{2}\right) \frac{\left(\not_{2} \not \gamma-\not q_{2}\right)}{4 M} u\left(p_{1}, s_{1}\right)\right]\right\},
\end{aligned}
$$

with the convolution integrals

$$
\begin{aligned}
\mathcal{H}_{W C}^{+(-)}(\xi, t) & \equiv \int_{-1}^{1} \frac{d x}{(x-\xi+i 0)}\left[Q_{+(-)}\left(H_{u}^{+}-H_{d}^{+}\right)+Q_{-(+)}\left(H_{u}^{-}-H_{d}^{-}\right)\right], \\
\mathcal{E}_{W C}^{+(-)}(\xi, t) & \equiv \int_{-1}^{1} \frac{d x}{(x-\xi+i 0)}\left[Q_{+(-)}\left(E_{u}^{+}-E_{d}^{+}\right)+Q_{-(+)}\left(E_{u}^{-}-E_{d}^{-}\right)\right],
\end{aligned}
$$




$$
\begin{aligned}
\tilde{\mathcal{H}}_{W C}^{+(-)}(\xi, t) & \equiv \int_{-1}^{1} \frac{d x}{(x-\xi+i 0)}\left[Q_{+(-)}\left(\tilde{H}_{u}^{+}-\tilde{H}_{d}^{+}\right)+Q_{-(+)}\left(\tilde{H}_{u}^{-}-\tilde{H}_{d}^{-}\right)\right] \\
\tilde{\mathcal{E}}_{W C}^{+(-)}(\xi, t) & \equiv \int_{-1}^{1} \frac{d x}{(x-\xi+i 0)}\left[Q_{+(-)}\left(\tilde{E}_{u}^{+}-\tilde{E}_{d}^{+}\right)+Q_{-(+)}\left(\tilde{E}_{u}^{-}-\tilde{E}_{d}^{-}\right)\right]
\end{aligned}
$$

The weak charged hadron tensor,

$$
\begin{aligned}
H_{W C}^{\nu \beta}= & -\frac{1}{2} \mathcal{T}_{W C}^{\mu \nu}\left(\mathcal{T}_{\mu W C}^{\beta}\right)^{*} \\
= & -\frac{1}{4}\left\{\left[\left(1-\xi^{2}\right)\left(\left|\mathcal{H}_{W C}^{+}\right|^{2}+\left|\mathcal{H}_{W C}^{-}\right|^{2}+\left|\tilde{\mathcal{H}}_{W C}^{+}\right|^{2}+\left|\tilde{\mathcal{H}}_{W C}^{-}\right|^{2}\right)\right.\right. \\
& -\left(\xi^{2}+\frac{t}{4 M^{2}}\right)\left(\left|\mathcal{E}_{W C}^{+}\right|^{2}+\left|\mathcal{E}_{W C}^{-}\right|^{2}\right)-\xi^{2} \frac{t}{4 M^{2}}\left(\left|\tilde{\mathcal{E}}_{W C}^{+}\right|^{2}+\left|\tilde{\mathcal{E}}_{W C}^{-}\right|^{2}\right) \\
& \left.-2 \xi^{2} \Re\left(\mathcal{H}_{W C}^{+*} \mathcal{E}_{W C}^{+}+\mathcal{H}_{W C}^{-*} \mathcal{E}_{W C}^{-}+\tilde{\mathcal{H}}_{W C}^{+*} \tilde{\mathcal{E}}_{W C}^{+}+\tilde{\mathcal{H}}_{W C}^{-*} \tilde{\mathcal{E}}_{W C}^{-}\right)\right] \\
& \times\left[g^{\nu \beta}-\frac{1}{\left(p \cdot q_{2}\right)}\left(p^{\nu} q_{2}^{\beta}+p^{\nu} q_{2}^{\beta}\right)+\frac{M^{2}}{\left(p \cdot q_{2}\right)^{2}}\left(1-\frac{t}{4 M^{2}}\right) q_{2}^{\nu} q_{2}^{\beta}\right] \\
& +2\left[\left(1-\xi^{2}\right) \Re\left(\mathcal{H}_{W C}^{+*} \mathcal{H}_{W C}^{-}+\tilde{\mathcal{H}}_{W C}^{+*} \tilde{\mathcal{H}}_{W C}^{-}\right)\right. \\
& -\left(\xi^{2}+\frac{t}{4 M^{2}}\right) \Re\left(\mathcal{E}_{W C}^{+*} \mathcal{E}_{W C}^{-}\right)-\xi^{2} \frac{t}{4 M^{2}} \Re\left(\tilde{\mathcal{E}}_{W C}^{+*} \tilde{\mathcal{E}}_{W C}^{-}\right) \\
& \left.-\xi^{2} \Re\left(\mathcal{H}_{W C}^{+*} \mathcal{E}_{W C}^{-}+\mathcal{E}_{W C}^{+*} \mathcal{H}_{W C}^{-}+\tilde{\mathcal{H}}_{W C}^{+*} \tilde{\mathcal{E}}_{W C}^{-}+\tilde{\mathcal{E}}_{W C}^{+*} \tilde{\mathcal{H}}_{W C}^{-}\right)\right] \\
& \left.\times \frac{1}{\left(p \cdot q_{2}\right)} i \epsilon^{\nu \beta \delta \lambda} p_{\delta} q_{2 \lambda}\right\},
\end{aligned}
$$

has identical structure as the expression (263) for the weak neutral hadron tensor. Moreover, by neglecting the mass of the outgoing muon, one can substitute the socalled muon tensor $L_{\nu \beta}^{(\mu)}$ by the neutrino tensor $L_{\nu \beta}^{(\nu)}$, see Eq. (262). The unpolarized Compton differential cross section for the weak charged DVCS process on a neutron target using the neutrino beam is

$$
\frac{d^{4} \sigma_{C \nu n}}{d x_{B} d Q_{1}^{2} d t d \varphi}=\frac{1}{(2 \pi)^{3}} \frac{\alpha G_{F}^{2}}{16} \frac{1+x_{B}(M / \omega)}{M^{2} \omega^{2}[2+(M / \omega)] x_{B}\left[y+2 x_{B}(M / \omega)\right]^{2}} L_{\nu \beta}^{(\mu)} H_{W C}^{\nu \beta} .
$$

The cross section is presented in Fig. 45.

We turn our attention now to the Bethe-Heitler background. It is given only by the diagram (b) in Fig. 39. At the angle $\phi^{\prime}$, when the outgoing real photon is collinear with the scattered muon, one should expect a pole. The amplitude of the Bethe-Heitler contribution reads

$$
\begin{aligned}
\mathrm{T}_{B H \nu n}= & \sqrt{2}|e| G_{F} \epsilon_{\mu}^{*}\left(q_{2}\right) \bar{u}\left(k^{\prime}\right)\left[\frac{\gamma^{\mu}\left(\not \not^{\prime}+\not q_{2}\right) \gamma^{\nu}\left(1-\gamma_{5}\right)}{\left(k^{\prime}+q_{2}\right)^{2}}\right] u(k) \\
& \times\left\langle p\left(p_{2}, s_{2}\right)\left|J_{\nu}^{C C}(0)\right| n\left(p_{1}, s_{1}\right)\right\rangle .
\end{aligned}
$$




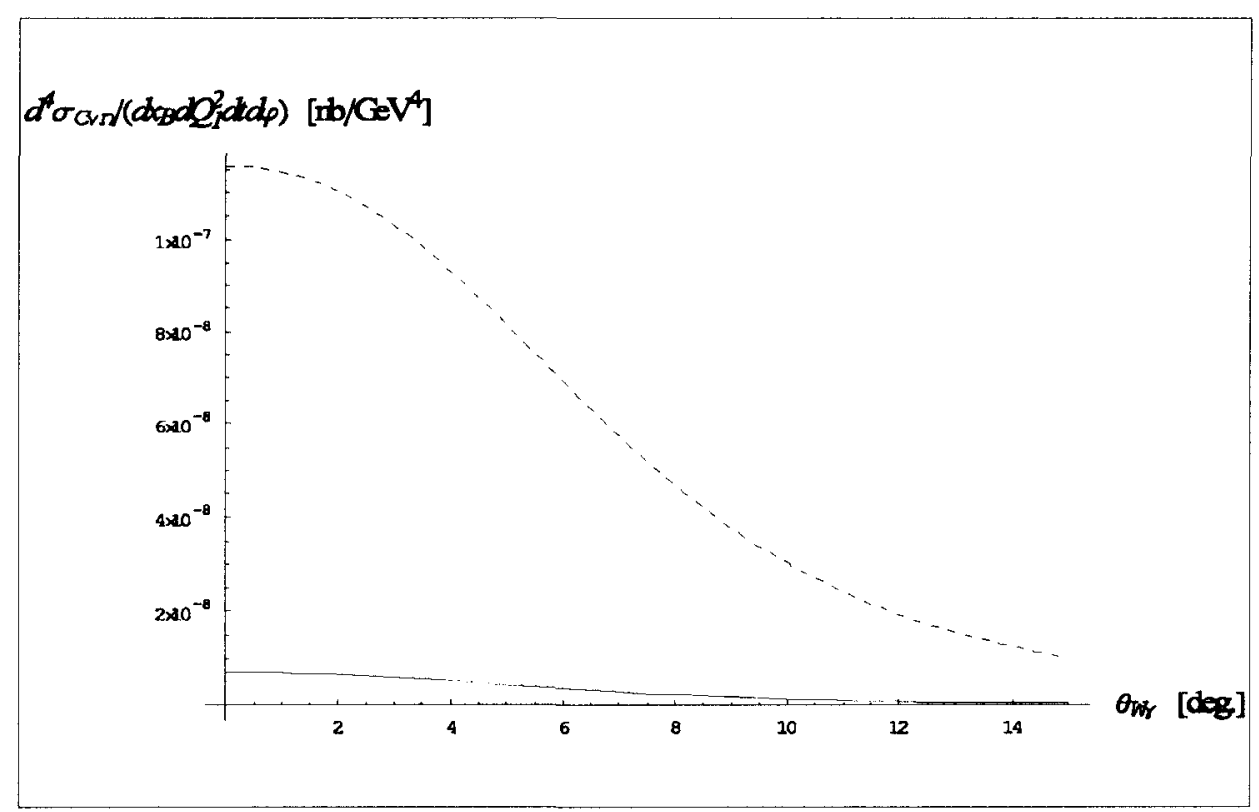

FIG. 45: Compton cross section $\sigma_{C \nu n}$ for the weak charged DVCS process plotted as a function of the angle $\theta_{B \gamma}$ between the incoming weak virtual boson and outgoing real photon in the target rest frame for $Q_{1}^{2}=2.5 \mathrm{GeV}^{2}$ and $x_{B}=0.35$ with $\omega=5 \mathrm{GeV}$ (solid line) and $\omega=20 \mathrm{GeV}$ (dashed line) neutrino beam.

The spin-averaged square of Eq. (285) is

$$
\overline{\left|\mathrm{T}_{B H \nu n}\right|^{2}}=8 \pi \alpha G_{F}^{2} L_{B H}^{\nu \beta} H_{\nu \beta}^{B H},
$$

with tensors

$$
\begin{aligned}
L_{B H}^{\nu \beta} & =\frac{8}{\left(k^{\prime} \cdot q_{2}\right)}\left[k^{\nu} q_{2}^{\beta}+k^{\beta} q_{2}^{\nu}-g^{\nu \beta}\left(k \cdot q_{2}\right)-i \epsilon^{\nu \beta \sigma \tau} k_{\sigma} q_{2 \tau}\right] \\
H_{\nu \beta}^{B H} & =\frac{1}{2} \sum_{s_{1}, s_{2}}\left\langle p\left(p_{2}, s_{2}\right)\left|J_{\nu}^{C C}(0)\right| n\left(p_{1}, s_{1}\right)\right\rangle\left\langle p\left(p_{2}, s_{2}\right)\left|J_{\beta}^{C C}(0)\right| n\left(p_{1}, s_{1}\right)\right\rangle^{*} .
\end{aligned}
$$

The matrix element of the weak charged transition current $J_{\nu}^{C C}$ between the nucleon states is defined as

$$
\left\langle p\left(p_{2}, s_{2}\right)\left|J_{\nu}^{C C}(0)\right| n\left(p_{1}, s_{1}\right)\right\rangle=\left\langle p\left(p_{2}, s_{2}\right)\left|\bar{\psi}_{p}(0) \gamma_{\nu} \frac{1}{2}\left(1-\gamma_{5}\right) \psi_{n}(0)\right| n\left(p_{1}, s_{1}\right)\right\rangle
$$

Using the same isospin symmetry relation between the flavor nondiagonal and flavor diagonal nucleon matrix elements, see Eq. (280), we parametrize the vector current 
part of the matrix element in Eq. (288) in terms of the Dirac and Pauli form factors for each quark flavor,

$$
\begin{array}{r}
\left\langle p\left(p_{2}, s_{2}\right)\left|\bar{\psi}_{p}(0) \gamma_{\nu} \psi_{n}(0)\right| n\left(p_{1}, s_{1}\right)\right\rangle=\left\langle p\left(p_{2}, s_{2}\right)\left|\bar{\psi}_{u}(0) \gamma_{\nu} \psi_{d}(0)\right| n\left(p_{1}, s_{1}\right)\right\rangle \\
=\left\langle p\left(p_{2}, s_{2}\right)\left|\bar{\psi}_{u}(0) \gamma_{\nu} \psi_{u}(0)\right| p\left(p_{1}, s_{1}\right)\right\rangle-\left\langle p\left(p_{2}, s_{2}\right)\left|\bar{\psi}_{d}(0) \gamma_{\nu} \psi_{d}(0)\right| p\left(p_{1}, s_{1}\right)\right\rangle \\
=\bar{u}\left(p_{2}, s_{2}\right)\left\{\left[F_{1 u}(t)-F_{1 d}(t)\right] \gamma_{\nu}-\left[F_{2 u}(t)-F_{2 d}(t)\right] \frac{i \sigma_{\nu \lambda} r^{\lambda}}{2 M}\right\} u\left(p_{1}, s_{1}\right) .
\end{array}
$$

Furthermore, for the axial vector current part we have

$$
\begin{aligned}
\left\langle p\left(p_{2}, s_{2}\right)\left|\bar{\psi}_{p}(0) \gamma_{\nu} \gamma_{5} \psi_{n}(0)\right| n\left(p_{1}, s_{1}\right)\right\rangle= & \bar{u}\left(p_{2}, s_{2}\right)\left[g_{A}(t) \gamma_{\nu} \gamma_{5}\right. \\
& \left.-g_{P}(t) \frac{\gamma_{5} r_{\nu}}{2 M}\right] u\left(p_{1}, s_{1}\right),
\end{aligned}
$$

where the $t$-dependence of the axial and pseudoscalar from factors is given by

$$
\begin{aligned}
& g_{A}(t)=g_{A}(t=0)\left(1-\frac{t}{m_{A}^{2}}\right)^{-2}, \\
& g_{P}(t)=g_{A}(t) \frac{4 M^{2}}{m_{\pi}^{2}-t} .
\end{aligned}
$$

Thus the unpolarized Bethe-Heitler differential cross section for the weak charged DVCS process is written in the form

$$
\frac{d^{4} \sigma_{B H \nu n}}{d x_{B} d Q_{1}^{2} d t d \varphi}=\frac{1}{(2 \pi)^{3}} \frac{\alpha G_{F}^{2}}{16} \frac{1+x_{B}(M / \omega)}{M^{2} \omega^{2}[2+(M / \omega)] x_{B}\left[y+2 x_{B}(M / \omega)\right]^{2}} L_{B H}^{\nu \beta} H_{\nu \beta}^{B H} .
$$

Its dependence on $\theta_{W \gamma}$ is plotted in Figs. 46 and 47, and together with the Compton cross section in Fig. 48. In particular, the plots in Fig. 48 reveal that, in the nearly forward direction, the Bethe-Heitler contamination is now suppressed by a factor $\simeq 1 / 10$ compared to the Compton contribution.

To summarize, we gave a comprehensive review on neutrino-induced DVCS processes. Through the weak interaction currents, these processes give access to two different sets of GPDs, namely, to the sum of quark and antiquark distributions and to their difference. Thus we can measure independently both the valence and sea content of GPDs. In addition, the weak charged current interaction probes GPDs that are nondiagonal in quark flavor, such as those associated with the neutron-to-proton transition. 


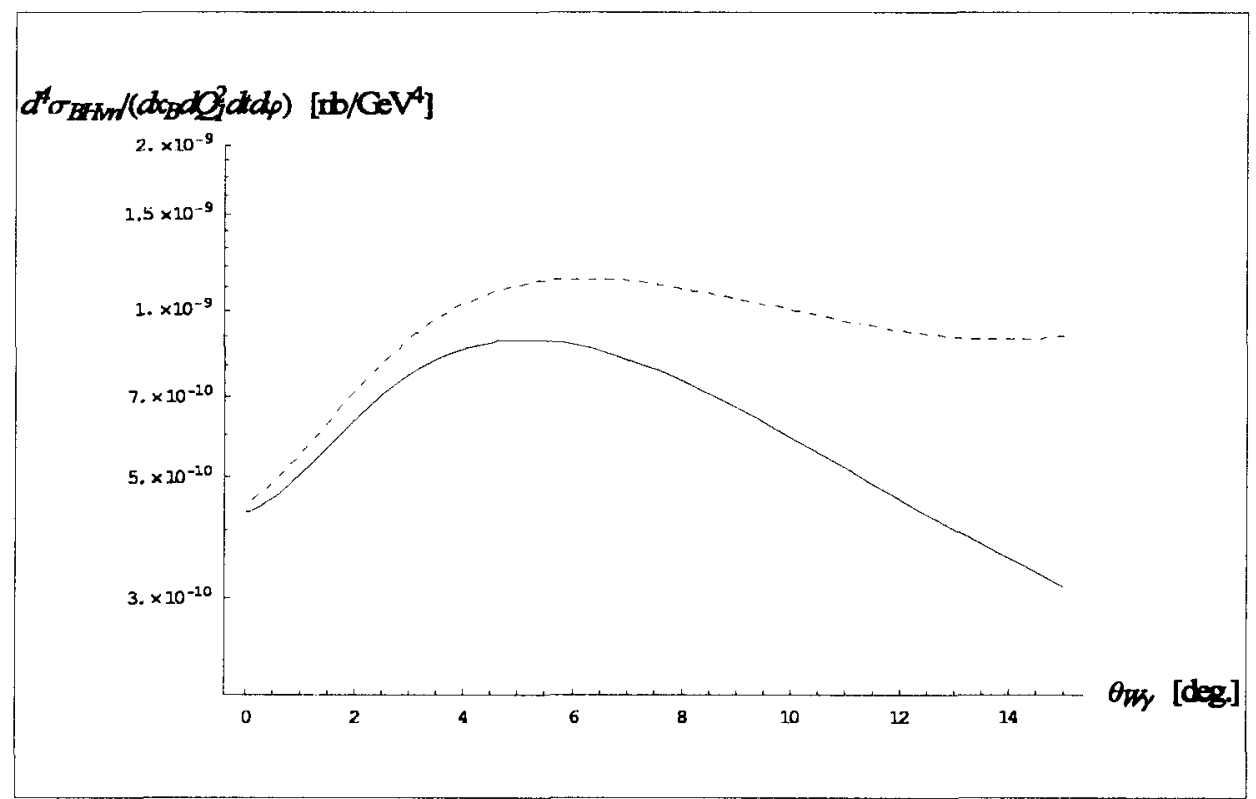

FIG. 46: Bethe-Heitler cross section $\sigma_{B H \nu n}$ for the weak charged DVCS process plotted as a function of the angle $\theta_{B \gamma}$ between the incoming weak virtual boson and outgoing real photon in the target rest frame for $Q_{1}^{2}=2.5 \mathrm{GeV}^{2}$ and $x_{B}=0.35$ with $\omega=5 \mathrm{GeV}$ (solid line) and $\omega=20 \mathrm{GeV}$ (dashed line) neutrino beam.

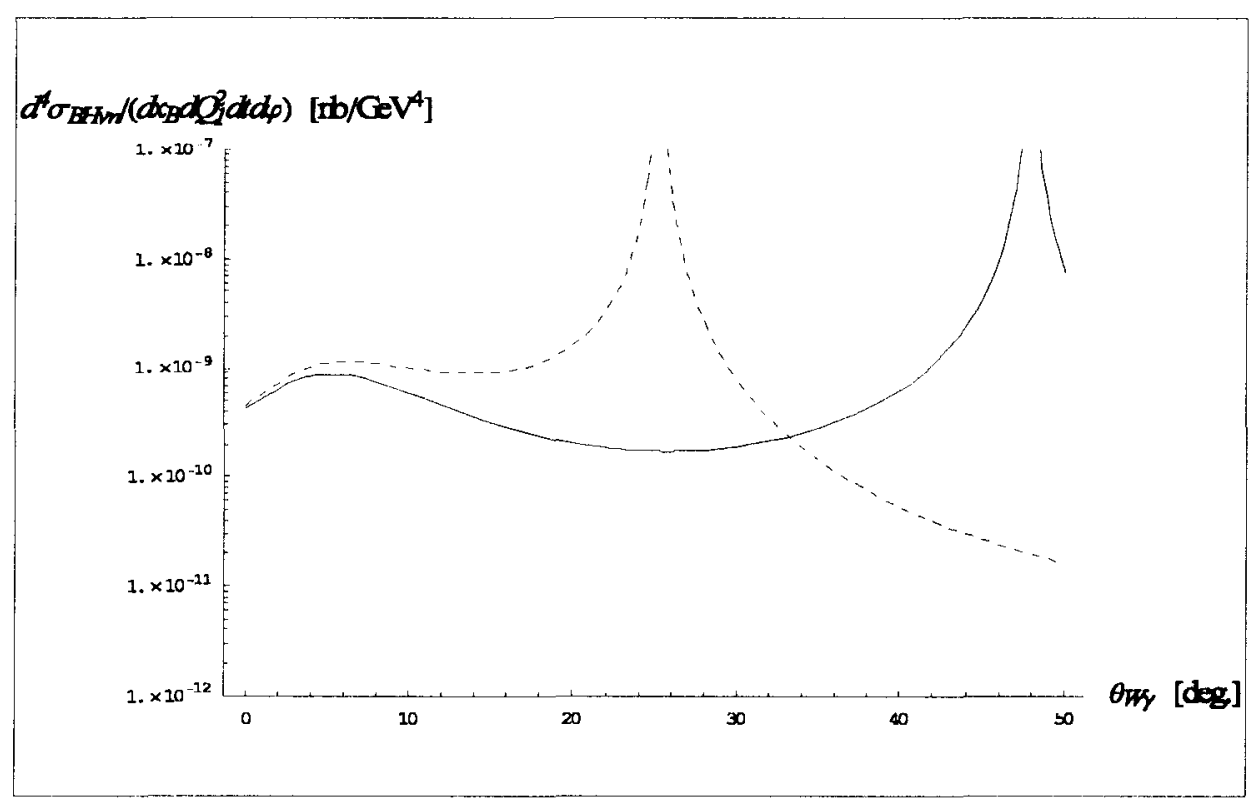

FIG. 47: Bethe-Heitler cross section $\sigma_{B H \nu n}$ for the weak charged DVCS process plotted as a function of the angle $\theta_{B \gamma}$ between the incoming weak virtual boson and outgoing real photon in the target rest frame for $Q_{1}^{2}=2.5 \mathrm{GeV}^{2}$ and $x_{B}=0.35$ with $\omega=5 \mathrm{GeV}$ (solid line) and $\omega=20 \mathrm{GeV}$ (dashed line) neutrino beam. 


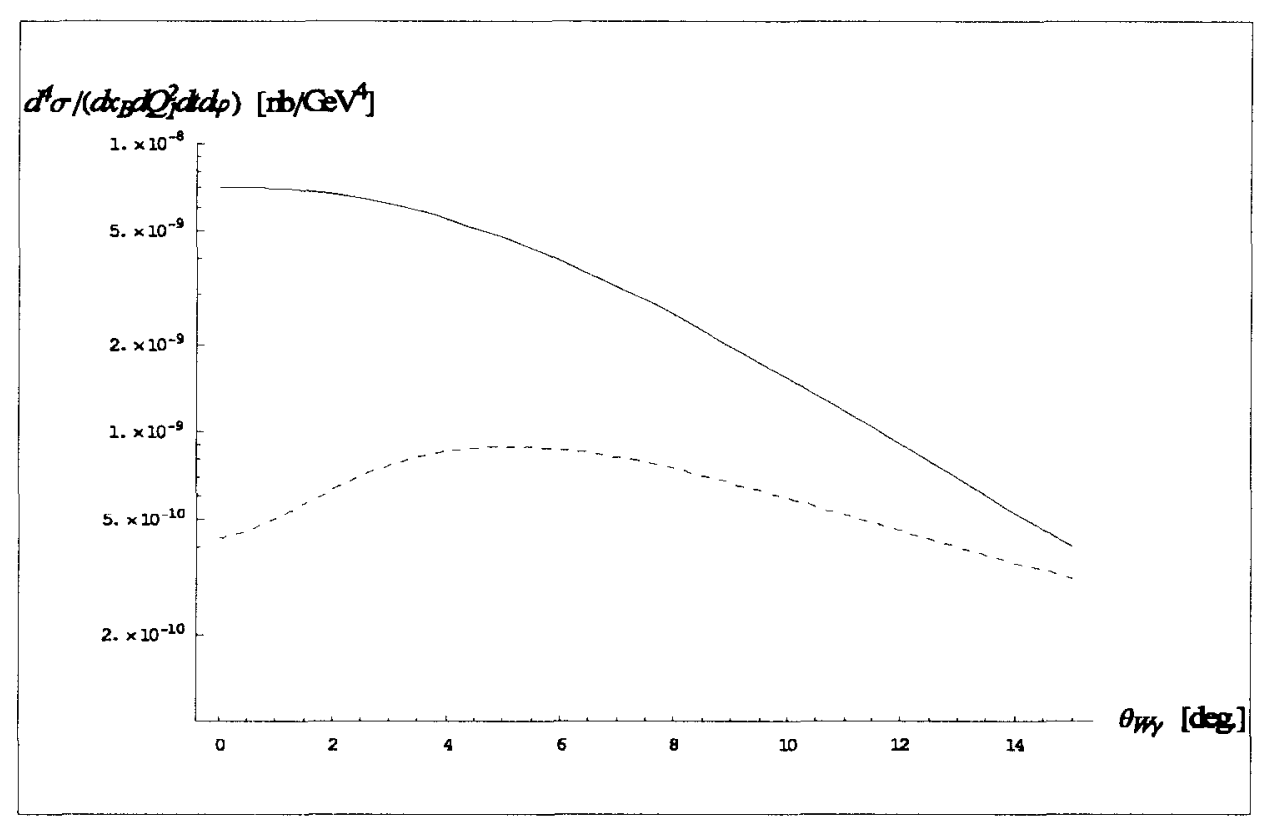

FIG. 48: Compton cross section $\sigma_{C \nu n}$ (solid line) and Bethe-Heitler cross section $\sigma_{B H \nu n}$ (dashed line) for the weak charged DVCS process plotted as a function of the angle $\theta_{B \gamma}$ between the incoming weak virtual boson and outgoing real photon in the target rest frame for $Q_{1}^{2}=2.5 \mathrm{GeV}^{2}$ and $x_{B}=0.35$ with $\omega=5 \mathrm{GeV}$ neutrino beam.

TABLE IV: Orders of magnitude (in nbarns) for the unpolarized total weak neutral $\sigma_{\nu p}$, Compton weak neutral $\sigma_{C e p}$, Compton weak charged $\sigma_{C \nu n}$ and Compton electromagnetic $\sigma_{C e p}$ cross sections for two different kinematical regions of the Bjorken scaling variable with two different lepton beam energies $\omega$.

\begin{tabular}{lccccc}
\hline \hline kinematical region & $\omega[\mathrm{GeV}]$ & $\sigma_{\nu p}\left(Z^{0}\right)$ & $\sigma_{C e p}\left(Z^{0}\right)$ & $\sigma_{C \nu n}\left(W^{+}\right)$ & $\sigma_{C e p}(\gamma)$ \\
\hline $0.28 \leq x_{B} \leq 0.3$ & 5 & $10^{-11}$ & $10^{-13}$ & $10^{-11}$ & $10^{-5}$ \\
$0.26 \leq x_{B} \leq 0.28$ & 20 & $10^{-9}$ & $10^{-10}$ & $10^{-9}$ & $10^{-3}$ \\
\hline \hline
\end{tabular}




\section{CHAPTER VIII}

\section{WIDE-ANGLE REAL COMPTON SCATTERING}

\section{VIII.1 INTRODUCTION}

Standard electromagnetic Compton scattering provides a unique tool for studying hadrons. We have demonstrated in the previous chapter how the process can be extended into the weak interaction sector, in which the relevant Compton amplitude probes the hadronic structure by means of the quark electromagnetic and weak currents rather than two electromagnetic currents, and promise therefore a new insight. In the lowest QCD approximation, these currents couple to photons and weak bosons through point-like vertices. Moreover, in a specific kinematical regime, featuring the presence of a large momentum transfer, the behavior of both the standard and weak Compton amplitudes is dominated by the light-like distances. As a result the amplitudes are given in terms of the handbag diagrams. There are several situations, where the handbag contribution plays an essential role [38]:

- Both initial and final photons are highly virtual and have equal space-like virtualities. This situation corresponds to the forward VCA. Its imaginary part determines the structure functions of DIS.

- The condition on photon virtualities may be relaxed in a sense that the initial photon is still far off-shell but the final photon is real and the invariant momentum transfer to the hadron is small. The situation corresponds to the nonforward VCA. It is accessible through DVCS.

- The configuration, in which both photons in the initial and final states are real but the invariant momentum transfer is large. The physical process corresponding to this situation is known as wide-angle real Compton scattering (WACS).

The Compton amplitude of WACS is a subject of the study in the present chapter, which contains some of the formal results from Ref. [111]. An efficient way to study this amplitude is again to use the light-cone expansion of the time-ordered product of two electromagnetic currents in the coordinate representation. At moderately large values of Mandelstam invariants $\left(s,-t,-u \leq 10 \mathrm{GeV}^{2}\right)$, the dominant reaction 
mechanism corresponds to the handbag contribution just as in DIS and DVCS. To the leading order, the amplitude is given by the $s$ - and $u$-channel handbag diagrams, see the diagrams (a) and (b) in Fig. 49. On the other hand, for extremely large $-t \gg 10 \mathrm{GeV}^{2}$ the purely hard contributions involving two-gluon exchange, such as the diagram (c) in Fig. 49, and having power-law behavior (i.e. $\sim(-t)^{N}$ ), become dominant. In particular, at large values of $t$, the handbag diagrams are accompanied by the Sudakov form factor $S(t) \sim \exp \left[-\alpha_{s} \ln ^{2}(-t) / 3 \pi\right]$ that drops faster than any power of $-t$. At moderate $t$, however, the hard contributions are numerically suppressed by a factor $\left(\alpha_{s} / \pi\right)^{2} \simeq 1 / 100$, and will not be considered here.

In Section VIII.2, we start from the formal light-cone expansion in terms of QCD string operators (with gauge links along the straight line between the fields which, for brevity, we do not write explicitly). In addition to the twist-2 string operators, we also include now the operators that are algebraically of twist- 3 but given by the total derivatives of twist-2 operators. One refers to these as the kinematical twist3 contributions, in order to distinguish them from the so-called dynamical twist-3 contributions involving quark-gluon operators. The latter cannot be reduced to total derivatives [83]. Furthermore, for simplicity, we consider a spin zero (i.e. pion) target. For that reason, only the matrix element of a vector-type string operator is needed. Its spectral representation is given in terms of double distributions. It is worth noting at this point that even though we restrict ourselves to the pion, our approach can be used in more realistic situation involving the nucleon target [112] as well as can be applied to the time-like region, in particular, to study the exclusive production of two pions in the two-photon collisions [113]. In Section VIII.3, we compute the Compton amplitude for the pion up to the twist-3 level, and inspect its transversality, in other words, the electromagnetic gauge invariance with respect to both initial and final photons. The physical amplitudes (or alternatively the photon helicity amplitudes) of the WACS process are obtained by projecting the Compton amplitude on different the polarization states of physical photons. We estimate these amplitudes and their corresponding cross sections by taking a simple model for the double distribution of the pion. The results are compared with the QED calculation. 


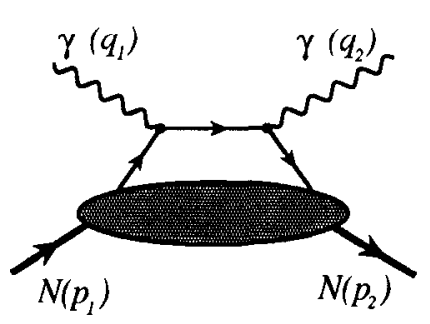

a

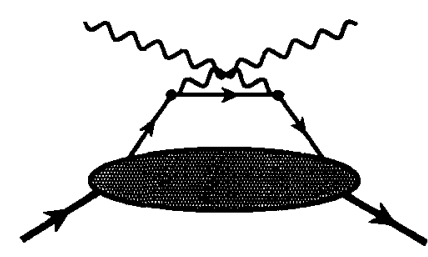

b

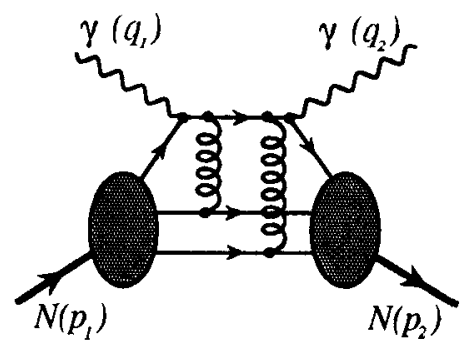

c

FIG. 49: Handbag contribution ( $\mathrm{a}$ and $\mathrm{b}$ ) and the configuration (c) with the exchange of two hard gluons.

\section{VIII.2 TWIST DECOMPOSITION AND PARAMETRIZATION}

We recall from Section IV.2 that the leading light-cone singularity of the time-ordered product of two electromagnetic currents is contained in the handbag contribution,

$$
i T\left\{J_{E M}^{\mu}(z / 2) J_{E M}^{\nu}(-z / 2)\right\}=\frac{z_{\rho}}{2 \pi^{2} z^{4}} \sum_{f} Q_{f}^{2}\left\{s^{\mu \rho \nu \eta} \mathcal{O}_{\eta}^{f}(z \mid 0)+i \epsilon^{\mu \rho \nu \eta} \mathcal{O}_{5 \eta}^{f}(z \mid 0)\right\}
$$

in which a highly virtual quark propagator connecting the two photon vertices is convoluted with the blob describing the long-distance dynamics. In Eq. (293) the tensor $s^{\mu \rho \nu \eta} \equiv g^{\mu \rho} g^{\nu \rho}+g^{\mu \eta} g^{\rho \nu}-g^{\mu \nu} g^{\rho \eta}$ and the QCD bilocal operators with the flavor index $f$ and one open Lorentz index are

$$
\begin{aligned}
\mathcal{O}_{\eta}^{f}(z \mid 0) & =\left[\bar{\psi}_{f}(-z / 2) \gamma_{\eta} \psi_{f}(z / 2)-\bar{\psi}_{f}(z / 2) \gamma_{\eta} \psi_{f}(-z / 2)\right] \\
\mathcal{O}_{5 \eta}^{f}(z \mid 0) & =\left[\bar{\psi}_{f}(-z / 2) \gamma_{\eta} \gamma_{5} \psi_{f}(z / 2)+\bar{\psi}_{f}(z / 2) \gamma_{\eta} \gamma_{5} \psi_{f}(-z / 2)\right]
\end{aligned}
$$

The Taylor expansion of these operators in the relative distance $z$ involves covariant derivatives and yields local operators that are not symmetric in their indices. Nevertheless, it was shown in Refs. $[83,86]$ that $\mathcal{O}_{\eta}^{f}(z \mid 0)$ and $\mathcal{O}_{5 \eta}^{f}(z \mid 0)$ can be expressed in a compact form in terms of the contracted operators $\mathcal{O}^{f}(z) \equiv z^{\eta} \mathcal{O}_{\eta}^{f}(z \mid 0)$ and $\mathcal{O}_{5}^{f}(z) \equiv z^{\eta} \mathcal{O}_{5 \eta}^{f}(z \mid 0)$ that produce only symmetric operators, and quark-gluon string operators. Namely, the fully deconstructed vector and axial vector operators take the following form

$$
\mathcal{O}_{\eta}^{f}(z \mid 0)=\int_{0}^{1} d v\left\{\cos \left[\frac{i \bar{v} \kappa}{2}\right] \partial_{\eta}+\frac{i v}{2} \sin \left[\frac{i \bar{v} \kappa}{2}\right] \frac{(z \cdot \mathcal{D}) \mathcal{D}_{\eta}-\mathcal{D}^{2} z_{\eta}}{\kappa}\right\}
$$




$$
\begin{aligned}
& \times\left[\bar{\psi}_{f}(-v z / 2) \not z \psi_{f}(v z / 2)-\bar{\psi}_{f}(v z / 2) \not \psi_{f}(-v z / 2)\right] \\
& +\epsilon_{\eta \delta \kappa \lambda} z^{\delta} \mathcal{D}_{\kappa} \int_{0}^{1} \frac{d v}{\kappa} \sin \left[\frac{i \bar{v} \kappa}{2}\right] \partial^{\lambda} \\
& \times\left[\bar{\psi}_{f}(-v z / 2) \not \gamma_{5} \psi_{f}(v z / 2)+\bar{\psi}_{f}(v z / 2) \not \gamma_{5} \psi_{f}(-v z / 2)\right] \\
& +(\text { quark-gluon operators }), \\
\mathcal{O}_{5 \eta}^{f}(z \mid 0)= & \int_{0}^{1} d v\left\{\cos \left[\frac{i \bar{v} \kappa}{2}\right] \partial_{\eta}+\frac{i v}{2} \sin \left[\frac{i \bar{v} \kappa}{2}\right] \frac{(z \cdot \mathcal{D}) \mathcal{D}_{\eta}-\mathcal{D}^{2} z_{\eta}}{\kappa}\right\} \\
& \times\left[\bar{\psi}_{f}(-v z / 2) \not \gamma_{5} \psi_{f}(v z / 2)+\bar{\psi}_{f}(v z / 2) \not \gamma_{5} \psi_{f}(-v z / 2)\right] \\
& +\epsilon_{\eta \delta \kappa \lambda} z^{\delta} \mathcal{D}_{\kappa} \int_{0}^{1} \frac{d v}{\kappa} \sin \left[\frac{i \bar{v} \kappa}{2}\right] \partial^{\lambda} \\
& \times\left[\bar{\psi}_{f}(-v z / 2) \not \psi_{f}(v z / 2)-\bar{\psi}_{f}(v z / 2) \not z \psi_{f}(-v z / 2)\right] \\
& +(\text { quark-gluon operators }),
\end{aligned}
$$

where we use the notation $\bar{v} \equiv 1-v, \partial_{\eta} \equiv \partial / \partial z^{\eta}$ and

$$
\kappa \equiv \sqrt{(z \cdot \mathcal{D})^{2}-z^{2} \mathcal{D}^{2}}
$$

with $\mathcal{D} \equiv \partial / \partial X$ being the total derivative. Neglecting $z^{2}$ terms and ignoring quarkgluon operators (note that they correspond to the dynamical twist-3 contributions, which are not considered in our approach) the decomposition (295) gives [81, 83, 86]

$$
\begin{aligned}
\mathcal{O}_{\eta}^{f}(z \mid 0)= & \int_{0}^{1} d v\left\{\cos \left[\frac{i \bar{v}(z \cdot \mathcal{D})}{2}\right] \partial_{\eta}+\frac{i v}{2} \sin \left[\frac{i \bar{v}(z \cdot \mathcal{D})}{2}\right] \mathcal{D}_{\eta}\right\} \\
& \times\left[\bar{\psi}_{f}(-v z / 2) \not z \psi_{f}(v z / 2)-\bar{\psi}_{f}(v z / 2) \not z \psi_{f}(-v z / 2)\right] \\
& +\epsilon_{\eta \delta \kappa \lambda} z^{\delta} \mathcal{D}_{\kappa} \int_{0}^{1} \frac{d v}{(z \cdot \mathcal{D})} \sin \left[\frac{i \bar{v}(z \cdot \mathcal{D})}{2}\right] \partial^{\lambda} \\
& \times\left[\bar{\psi}_{f}(-v z / 2) \not z \gamma_{5} \psi_{f}(v z / 2)+\bar{\psi}_{f}(v z / 2) \not \partial \gamma_{5} \psi_{f}(-v z / 2)\right], \\
\mathcal{O}_{5 \eta}^{f}(z \mid 0)= & \int_{0}^{1} d v\left\{\cos \left[\frac{i \bar{v}(z \cdot \mathcal{D})}{2}\right] \partial_{\eta}+\frac{i v}{2} \sin \left[\frac{i \bar{v}(z \cdot \mathcal{D})}{2}\right] \mathcal{D}_{\eta}\right\} \\
& \times\left[\bar{\psi}_{f}(-v z / 2) \not z \gamma_{5} \psi_{f}(v z / 2)+\bar{\psi}_{f}(v z / 2) \not \gamma_{5} \psi_{f}(-v z / 2)\right] \\
& +\epsilon_{\eta \delta \kappa \lambda} z^{\delta} \mathcal{D}_{\kappa} \int_{0}^{1} \frac{d v}{(z \cdot \mathcal{D})} \sin \left[\frac{i \bar{v}(z \cdot \mathcal{D})}{2}\right] \partial^{\lambda} \\
& \times\left[\bar{\psi}_{f}(-v z / 2) \not z \psi_{f}(v z / 2)-\bar{\psi}_{f}(v z / 2) \not \psi_{f}(-v z / 2)\right] .
\end{aligned}
$$

For the pion, the matrix element of the contracted axial vector operator vanishes and accordingly, the matrix element of the time-ordered product (293) reduces to

$$
\left\langle\pi\left(p_{2}\right)\left|i T\left\{J_{E M}^{\mu}(z / 2) J_{E M}^{\nu}(-z / 2)\right\}\right| \pi\left(p_{1}\right)\right\rangle=
$$




$$
\begin{array}{r}
\frac{z_{\rho}}{2 \pi^{2} z^{4}} \sum_{f} Q_{f}^{2}\left\{s^{\mu \rho \nu \eta} \int_{0}^{1} d v\left\{\cos \left[\frac{\bar{v}(r \cdot z)}{2}\right] \partial_{\eta}+\frac{v}{2} \sin \left[\frac{\bar{v}(r \cdot z)}{2}\right] r_{\eta}\right\}\right. \\
\left.+i \epsilon^{\mu \nu \eta \rho} \epsilon_{\eta \delta \kappa \lambda} z^{\delta} r^{\kappa} \int_{0}^{1} \frac{d v}{(r \cdot z)} \sin \left[\frac{\bar{v}(r \cdot z)}{2}\right] \partial^{\lambda}\right\} \\
\times\left\langle\pi\left(p_{2}\right)\left|\bar{\psi}_{f}(-v z / 2) \not z \psi_{f}(v z / 2)-\bar{\psi}_{f}(v z / 2) \not \partial \psi_{f}(-v z / 2)\right| \pi\left(p_{2}\right)\right\rangle .
\end{array}
$$

We have used the fact that under the matrix element, the total derivative of the string operator turns into the momentum transfer, $i \partial / \partial X_{\kappa} \rightarrow r^{\kappa}$ (recall that $r \equiv p_{1}-p_{2}$ is the overall momentum transfer to the pion target).

The next step is to parametrize the nonforward matrix element of the twist-2 part of the contracted vector string operator in Eq. (298). This is performed in terms of the spectral functions, namely, the double distributions. Thus one needs to construct the parametrization for the matrix element of $\mathcal{O}^{f}(z)$ satisfying the harmonic condition

$$
\partial^{2}\left\langle\pi\left(p_{2}\right)\left|\mathcal{O}^{f}(z)\right| \pi\left(p_{1}\right)\right\rangle_{t w i s t-2}=0
$$

In general, the matrix element can be regarded as a function of the variables $(p \cdot z)$, $(r \cdot z)$ and $z^{2}$, where $p \equiv\left(p_{1}+p_{2}\right) / 2$ is the average pion momentum. The standard way to parametrize it, is by the decomposition into plane waves, where one explicitly separates the $(p \cdot z)$ components from the $(r \cdot z)$ components, and hence use two spectral functions, $f_{f}(\beta, \alpha, t)$ and $g_{f}(\beta, \alpha, t)$. Alternatively, one can write the parametrization in the form

$$
\left\langle\pi(p-r / 2)\left|\mathcal{O}^{f}(z)\right| \pi(p+r / 2)\right\rangle_{t w i s t-2}=-2 i\left(z^{\sigma} \partial_{\sigma}\right) \mathcal{F}(z, p, r)
$$

with

$$
\mathcal{F}(z, p, r)=\int_{-1}^{1} d \beta \int_{-1+|\beta|}^{1-|\beta|} d \alpha e^{i(k \cdot z)}\left[h_{f}(\beta, \alpha, t)+\mathcal{O}\left(k^{2} z^{2}\right)\right],
$$

where the four-momentum $k=\beta p+\alpha r / 2$. The latter has the same structure as that parametrizing the twist-2 part of the matrix element $\langle p-r / 2|\phi(-z / 2) \phi(z / 2)| p+r / 2\rangle$ in the scalar case. In particular, it satisfies $\partial^{2} \mathcal{F}(z, p, r)=0$. It is easy to see that for that reason

$$
\begin{aligned}
\partial^{2}\left(z^{\sigma} \partial_{\sigma}\right) \mathcal{F}(z, p, r) & =\left(2+z^{\sigma} \partial_{\sigma}\right) \partial^{2} \mathcal{F}(z, p, r) \\
& =0
\end{aligned}
$$

Let us note at this point that in Appendix $\mathrm{C}$, we consider the scalar parametrization $\mathcal{F}(z, p, r)$, which includes terms up to order $\mathcal{O}\left(k^{4} z^{4}\right)$. The inclusion of $z^{2}$ and $z^{4}$ terms 
in the expansion is due to the fact that the fermion propagator has $1 / z^{4}$ singularity, and besides the operator $\mathcal{O}^{f}(v z)$ in Eq. (298) is differentiated with respect to $z$. Now, by taking only the first term in the $z^{2}$ expansion in $\mathcal{F}(z, p, r)$, the expression (300) turns into

$$
\begin{aligned}
\left\langle\pi(p-r / 2)\left|\mathcal{O}^{f}(z)\right| \pi(p+r / 2)\right\rangle_{t w i s t-2}= & 2 \int_{-1}^{1} d \beta \int_{-1+|\beta|}^{1-|\beta|} d \alpha e^{i(k \cdot z)} \\
& \times(k \cdot z) h_{f}(\beta, \alpha, t) .
\end{aligned}
$$

The representation (303) is given in terms of only one double distribution $h_{f}(\beta, \alpha, t)$ and corresponds to the so-called single-DD parametrization [88]. We observe that the original distributions are then expressed in terms of the single one as

$$
\begin{aligned}
& f_{f}(\beta, \alpha, t)=\beta h_{f}(\beta, \alpha, t) \\
& g_{f}(\beta, \alpha, t)=\alpha h_{f}(\beta, \alpha, t)
\end{aligned}
$$

Moreover, we establish the symmetry properties of $h_{f}(\beta, \alpha, t)$ with respect to parameters $\beta$ and $\alpha$. Since $f_{f}(\beta, \alpha, t)$ is odd in $\beta$ and even in $\alpha$ while for $g_{f}(\beta, \alpha, t)$ the situation is the other way around, it follows from Eq. (304) that the double distribution $h_{f}(\beta, \alpha, t)$ is an even function in both variables $\beta$ and $\alpha$.

Finally, by substituting the single-DD parametrization (303) into the matrix element (298), one arrives at

$$
\begin{array}{r}
\left\langle\pi\left(p_{2}\right)\left|i T\left\{J_{E M}^{\mu}(z / 2) J_{E M}^{\nu}(-z / 2)\right\}\right| \pi\left(p_{1}\right)\right\rangle= \\
\frac{z_{\rho}}{\pi^{2} z^{4}} \sum_{f} Q_{f}^{2} \int_{-1}^{1} d \beta \int_{-1+|\beta|}^{1-|\beta|} d \alpha h_{f}(\beta, \alpha, t) \int_{0}^{1} d v e^{i v(k \cdot z)} \\
\times\left\{s^{\mu \rho \nu \eta}\left\{\cos \left[\frac{\bar{v}(r \cdot z)}{2}\right]\left[k_{\eta}+i v k_{\eta}(k \cdot z)\right]+\frac{v}{2} r_{\eta}(k \cdot z) \sin \left[\frac{\bar{v}(r \cdot z)}{2}\right]\right\}\right. \\
\left.+i \epsilon^{\mu \nu \eta \rho} \epsilon_{\eta \delta \kappa \lambda} z^{\delta} r^{\kappa} \frac{1}{(r \cdot z)} \sin \left[\frac{\bar{v}(r \cdot z)}{2}\right]\left[k^{\lambda}+i v k^{\lambda}(k \cdot z)\right]\right\} .
\end{array}
$$

To isolate the leading twist-2 contribution in Eq. (305), we integrate by parts over $v$ the first $k_{\eta}$ term in the symmetric part (with respect to the Lorentz indices $\mu$ and $\nu$ ) of the matrix element, and the first $k^{\lambda}$ term in the antisymmetric part. We find

$$
\begin{array}{r}
\left\langle\pi\left(p_{2}\right)\left|i T\left\{J_{E M}^{\mu}(z / 2) J_{E M}^{\nu}(-z / 2)\right\}\right| \pi\left(p_{1}\right)\right\rangle= \\
\frac{z_{\rho}}{\pi^{2} z^{4}} \sum_{f} Q_{f}^{2} \int_{-1}^{1} d \beta \int_{-1+|\beta|}^{1-|\beta|} d \alpha h_{f}(\beta, \alpha, t) \\
\times\left\{s^{\mu \rho \nu \eta}\left\{k_{\eta} e^{i(k \cdot z)}+\frac{1}{2}\left[r_{\eta}(k \cdot z)-k_{\eta}(r \cdot z)\right] \int_{0}^{1} d v v e^{i v(k \cdot z)} \sin \left[\frac{\bar{v}(r \cdot z)}{2}\right]\right\}\right.
\end{array}
$$




$$
\left.+\frac{i}{2} \epsilon^{\mu \nu \eta \rho} \epsilon_{\eta \delta \kappa \lambda} z^{\delta} r^{\kappa} k^{\lambda} \int_{0}^{1} d v v e^{i v(k \cdot z)} \cos \left[\frac{\bar{v}(r \cdot z)}{2}\right]\right\}
$$

where only the surface term in the symmetric part corresponds to the twist-2 contribution (note that the surface term in the antisymmetric part vanishes). All the remaining terms are of the twist-3. We explicitly write

$$
\begin{aligned}
& \left\langle\pi\left(p_{2}\right)\left|i T\left\{J_{E M}^{\mu}(z / 2) J_{E M}^{\nu}(-z / 2)\right\}\right| \pi\left(p_{1}\right)\right\rangle_{t w i s t-2}= \\
& \frac{z_{\rho}}{\pi^{2} z^{4}} \sum_{f} Q_{f}^{2} s^{\mu \rho \nu \eta} \int_{-1}^{1} d \beta \int_{-1+|\beta|}^{1-|\beta|} d \alpha h_{f}(\beta, \alpha, t) k_{\eta} e^{i(k \cdot z)}
\end{aligned}
$$

for the twist-2 part. Now for the twist-3 part, after expressing the sine and cosine functions in Eq. (306) in terms of the exponentials and combining them with the overall exponential factor $\exp [i v(k \cdot z)]$, we obtain

$$
\begin{array}{r}
\left\langle\pi\left(p_{2}\right)\left|i T\left\{J_{E M}^{\mu}(z / 2) J_{E M}^{\nu}(-z / 2)\right\}\right| \pi\left(p_{1}\right)\right\rangle_{t w i s t-3}= \\
\frac{i z_{\rho}}{4 \pi^{2} z^{4}} \sum_{f} Q_{f}^{2} \int_{-1}^{1} d \beta \int_{-1+|\beta|}^{1-|\beta|} d \alpha h_{f}(\beta, \alpha, t) \\
\times\left\{s^{\mu \rho \nu \eta}\left[r_{\eta}(k \cdot z)-k_{\eta}(r \cdot z)\right] \int_{0}^{1} d v v\left[e^{i v\left(k_{1} \cdot z\right)-i(r \cdot z) / 2}-e^{i v\left(k_{2} \cdot z\right)+i(r \cdot z) / 2}\right]\right. \\
\left.+\epsilon^{\mu \nu \eta \rho} \epsilon_{\eta \delta \kappa \lambda} z^{\delta} r^{\kappa} k^{\lambda} \int_{0}^{1} d v v\left[e^{i v\left(k_{1} \cdot z\right)-i(r \cdot z) / 2}+e^{i v\left(k_{2} \cdot z\right)+i(r \cdot z) / 2}\right]\right\}
\end{array}
$$

where $k_{1}=k+r / 2$ and $k_{2}=k-r / 2$ are the momenta of the incoming (with respect to the interaction with two photons) and outgoing quarks, respectively.

\section{VIII.3 COMPTON SCATTERING AMPLITUDE}

As a result of the twist decomposition of the matrix element, see Eqs. (307) and (308), the Compton scattering amplitude,

$$
\mathcal{T}^{\mu \nu}=\sum_{f} Q_{f}^{2} \int d^{4} z e^{i(q \cdot z)}\left\langle\pi\left(p_{2}\right)\left|i T\left\{J_{E M}^{\mu}(z / 2) J_{E M}^{\nu}(-z / 2)\right\}\right| \pi\left(p_{1}\right)\right\rangle,
$$

where $q \equiv\left(q_{1}+q_{2}\right) / 2$ is the average photon momentum, can be written in our approximation, as the sum of the twist-2 and twist- 3 contributions,

$$
\mathcal{T}^{\mu \nu}=\mathcal{T}_{t w i s t-2}^{\mu \nu}+\mathcal{T}_{t w i s t-3}^{\mu \nu}
$$




\section{VIII.3.1 Twist-2 Amplitude}

After integrating over $z$ with the help of Eq. (98), the twist-2 part of the amplitude reads

$$
\begin{aligned}
\mathcal{T}_{t w i s t-2}^{\mu \nu}= & \sum_{f} Q_{f}^{2} s^{\mu \rho \nu \eta} \int d^{4} z e^{i(k+q) \cdot z} \frac{z_{\rho}}{\pi^{2} z^{4}} \int_{-1}^{1} d \beta \int_{-1+|\beta|}^{1-|\beta|} d \alpha h_{f}(\beta, \alpha, t) k_{\eta} \\
= & 2 \sum_{f} Q_{f}^{2} \int_{-1}^{1} d \beta \int_{-1+|\beta|}^{1-|\beta|} d \alpha h_{f}(\beta, \alpha, t) \frac{1}{(k+q)^{2}} \\
& \times\left\{2 k^{\mu} k^{\nu}+k^{\mu} q^{\nu}+k^{\nu} q^{\mu}-g^{\mu \nu}\left[k^{2}+(k \cdot q)\right]\right\} .
\end{aligned}
$$

If the pion mass $m_{\pi}$ is neglected then $k^{2}=-\left(\beta^{2}-\alpha^{2}\right) t / 4$ and the denominator for the quark propagator results into

$$
\begin{aligned}
(k+q)^{2} & =\left(k_{1}+q_{1}\right)^{2} \\
& =\left(k_{2}+q_{2}\right)^{2} \\
& =s\left\{\beta-\frac{t}{4 s}\left[(1-\beta)^{2}-\alpha^{2}\right]\right\},
\end{aligned}
$$

where $s=2(p \cdot q)-t / 2=2\left(p_{1} \cdot q_{1}\right)=2\left(p_{2} \cdot q_{2}\right)$ or, after writing $(1-\beta)^{2}=$ $(1-|\beta|)^{2}+2(|\beta|-\beta)$

$$
(k+q)^{2}=\left\{\begin{array}{cc}
\beta s-\left[(1-\beta)^{2}-\alpha^{2}\right] t / 4 & \text { for } \beta>0 \\
-\beta u-\left[(1+\beta)^{2}-\alpha^{2}\right] t / 4 & \text { for } \beta<0
\end{array}\right.
$$

\section{VIII.3.2 Twist-3 Amplitude}

We compute now the twist-3 amplitude, which contains two contributions. First, the contribution from the $s^{\mu \rho \nu \eta}$ term in Eq. (308) is

$$
\begin{aligned}
\mathcal{T}_{t w i s t-3(1)}^{\mu \nu}= & \frac{1}{2} \sum_{f} Q_{f}^{2} s^{\mu \rho \nu \eta} \int_{-1}^{1} d \beta \int_{-1+|\beta|}^{1-|\beta|} d \alpha h_{f}(\beta, \alpha, t) \\
& \times \int_{0}^{1} d v v \int d^{4} z\left[e^{i\left(l_{1} \cdot z\right)}-e^{i\left(l_{2} \cdot z\right)}\right] \frac{i z_{\rho}}{2 \pi^{2} z^{4}}\left[r_{\eta}(k \cdot z)-k_{\eta}(r \cdot z)\right],
\end{aligned}
$$

where the combined exponentials, $l_{1} \equiv q_{1}+v k_{1}$ and $l_{2} \equiv q_{2}+v k_{2}$, correspond to the effective momenta flowing through the quark propagator, and $q_{1}=q-r / 2$ and $q_{2}=q+r / 2$ are the momenta of the initial and final photons. We have two types of quark propagators here. Their denominators are given by

$$
l_{1}^{2}=v\left[\left(k_{1} \cdot q_{1}\right)+v k_{1}^{2}\right]
$$




$$
\begin{aligned}
& =v\left[(k+q)^{2}-\bar{v} k_{1}^{2}\right] \\
l_{2}^{2} & =v\left[\left(k_{2} \cdot q_{2}\right)+v k_{2}^{2}\right] \\
& =v\left[(k+q)^{2}-\bar{v} k_{2}^{2}\right],
\end{aligned}
$$

where the incoming and outgoing quark virtualities are

$$
\begin{aligned}
& k_{1}^{2}=\left[-\beta^{2}+(1+\alpha)^{2}\right] t / 4, \\
& k_{2}^{2}=\left[-\beta^{2}+(1-\alpha)^{2}\right] t / 4 .
\end{aligned}
$$

After the integral over $z$ is carried out using of the formula

$$
\int d^{4} z e^{i(l \cdot z)} \frac{i z_{\rho} z_{\sigma}}{2 \pi^{2}\left(z^{2}-i 0\right)^{2}}=\frac{g_{\rho \sigma} l^{2}-2 l_{\rho} l_{\sigma}}{\left(l^{2}+i 0\right)^{2}}
$$

the expression (314) turns into

$$
\begin{aligned}
\mathcal{T}_{t w i s t-3(1)}^{\mu \nu}= & \sum_{f} Q_{f}^{2} \int_{-1}^{1} d \beta \int_{-1+|\beta|}^{1-|\beta|} d \alpha h_{f}(\beta, \alpha, t) \int_{0}^{1} d v v \\
& \times\left\{\frac{1}{l_{1}^{4}}\left[-\left(l_{1} \cdot k\right)\left(l_{1}^{\mu} r^{\nu}+l_{1}^{\nu} r^{\mu}\right)+\left(l_{1} \cdot r\right)\left(l_{1}^{\mu} k^{\nu}+l_{1}^{\nu} k^{\mu}\right)\right]\right. \\
& \left.-\frac{1}{l_{2}^{4}}\left[-\left(l_{2} \cdot k\right)\left(l_{2}^{\mu} r^{\nu}+l_{2}^{\nu} r^{\mu}\right)+\left(l_{2} \cdot r\right)\left(l_{2}^{\mu} k^{\nu}+l_{2}^{\nu} k^{\mu}\right)\right]\right\} .
\end{aligned}
$$

Next we study the second contribution to $\mathcal{T}_{t w i s t-3}^{\mu \nu}$. It is due to the $\epsilon^{\mu \nu \eta \rho}$ term in the matrix element (308, and comes from the axial vector operator. We have

$$
\begin{aligned}
\mathcal{T}_{t w i s t-3(2)}^{\mu \nu}= & \frac{1}{2} \sum_{f} Q_{f}^{2} \int_{-1}^{1} d \beta \int_{-1+|\beta|}^{1-|\beta|} d \alpha h_{f}(\beta, \alpha, t) \\
& \times \epsilon^{\mu \nu \eta \rho} \epsilon_{\eta \delta \kappa \lambda} r^{\kappa} k^{\lambda} \int_{0}^{1} d v v \int d^{4} z\left[e^{i\left(l_{1} \cdot z\right)}+e^{i\left(l_{2} \cdot z\right)}\right] \frac{i z_{\rho} z^{\delta}}{2 \pi^{2} z^{4}} \\
= & \sum_{f} Q_{f}^{2} \int_{-1}^{1} d \beta \int_{-1+|\beta|}^{1-|\beta|} d \alpha h_{f}(\beta, \alpha, t) \int_{0}^{1} d v v \\
& \left\{\frac{1}{l_{1}^{4}}\left[\left(l_{1} \cdot k\right)\left(l_{1}^{\mu} r^{\nu}-l_{1}^{\nu} r^{\mu}\right)-\left(l_{1} \cdot r\right)\left(l_{1}^{\mu} k^{\nu}-l_{1}^{\nu} k^{\mu}\right)\right]\right. \\
& \left.+\frac{1}{l_{2}^{4}}\left[\left(l_{2} \cdot k\right)\left(l_{2}^{\mu} r^{\nu}-l_{2}^{\nu} r^{\mu}\right)-\left(l_{2} \cdot r\right)\left(l_{2}^{\mu} k^{\nu}-l_{2}^{\nu} k^{\mu}\right)\right]\right\} .
\end{aligned}
$$

Finally, by adding Eqs. (318) and (319), the total twist-3 result reads

$$
\begin{aligned}
\mathcal{T}_{t w i s t-3}^{\mu \nu}= & 2 \sum_{f} Q_{f}^{2} \int_{-1}^{1} d \beta \int_{-1+|\beta|}^{1-|\beta|} d \alpha h_{f}(\beta, \alpha, t) \int_{0}^{1} d v v \\
& \times\left\{\frac{1}{l_{1}^{4}}\left[-\left(l_{1} \cdot k\right) l_{1}^{\nu} r^{\mu}+\left(l_{1} \cdot r\right) l_{1}^{\nu} k^{\mu}\right]+\frac{1}{l_{2}^{4}}\left[\left(l_{2} \cdot k\right) l_{2}^{\mu} r^{\nu}-\left(l_{2} \cdot r\right) l_{2}^{\mu} k^{\nu}\right]\right\}
\end{aligned}
$$




$$
\begin{aligned}
= & 2 \sum_{f} Q_{f}^{2} \int_{-1}^{1} d \beta \int_{-1+|\beta|}^{1-|\beta|} d \alpha h_{f}(\beta, \alpha, t) \\
& \times\left\{\int_{0}^{1} d v v^{2}\left[\frac{k_{1}^{\nu}}{l_{1}^{4}}\left[-\left(l_{1} \cdot k\right) r^{\mu}+\left(l_{1} \cdot r\right) k^{\mu}\right]+\frac{k_{2}^{\mu}}{l_{2}^{4}}\left[\left(l_{2} \cdot k\right) r^{\nu}-\left(l_{2} \cdot r\right) k^{\nu}\right]\right]\right. \\
& \left.+\int_{0}^{1} d v v\left[\frac{q_{1}^{\nu}}{l_{1}^{4}}\left[-\left(l_{1} \cdot k\right) r^{\mu}+\left(l_{1} \cdot r\right) k^{\mu}\right]+\frac{q_{2}^{\mu}}{l_{2}^{4}}\left[\left(l_{2} \cdot k\right) r^{\nu}-\left(l_{2} \cdot r\right) k^{\nu}\right]\right]\right\},
\end{aligned}
$$

with the following scalar products

$$
\begin{aligned}
& \left(l_{1} \cdot k\right)=(k+q)^{2} / 2+(v-1 / 2) k_{1}^{2}+(\bar{v}-v \alpha) t / 4, \\
& \left(l_{2} \cdot k\right)=(k+q)^{2} / 2+(v-1 / 2) k_{2}^{2}+(\bar{v}+v \alpha) t / 4, \\
& \left(l_{1} \cdot r\right)=-(\bar{v}-v \alpha) t / 2, \\
& \left(l_{2} \cdot r\right)=(\bar{v}+v \alpha) t / 2 .
\end{aligned}
$$

Note that the second integral over $v$ in the amplitude (320) is logarithmically divergent, however, the coefficient for the divergent integral vanishes, when $\mathcal{T}_{\text {twist-3 }}^{\mu \nu}$ is contracted with the incoming and outgoing photon polarization vectors, $\epsilon_{\nu}\left(q_{1}\right)$ and $\epsilon_{\mu}\left(q_{2}\right)$.

\section{VIII.3.3 Electromagnetic Gauge Invariance}

The total Compton scattering amplitude (309) is transverse with respect to incoming and outgoing photon momenta

$$
\begin{aligned}
& \mathcal{T}^{\mu \nu} q_{1 \nu}=0 \\
& q_{2 \mu} \mathcal{T}^{\mu \nu}=0
\end{aligned}
$$

Let us check whether the gauge invariant condition (322) also holds for the amplitude (310). We expand the contracted twist-2 and twist-3 amplitudes in terms of $t / s$ and, for simplicity, keep only the lowest (zeroth) order corrections. A straightforward contraction, together with imposing the symmetry properties of the double distribution $h_{f}(\beta, \alpha, t)$ with respect to the variables $\alpha$ and $\beta$, yields for the leading-twist amplitude

$$
\begin{aligned}
& \mathcal{T}_{\text {twist-2 }}^{\mu \nu} q_{1 \nu} \\
& \mathcal{T}_{\text {twist }-2}^{\mu \nu} q_{1 \nu}=\sum_{f} Q_{f}^{2} \int_{-1}^{1} d \beta \int_{-1+|\beta|}^{1-|\beta|} d \alpha h_{f}(\beta, \alpha, t) r^{\mu} / 2 \\
& q_{2 \mu} \mathcal{T}_{\text {twist-2 }}^{\mu \nu}=-\sum_{f} Q_{f}^{2} \int_{-1}^{1} d \beta \int_{-1+|\beta|}^{1-|\beta|} d \alpha h_{f}(\beta, \alpha, t) r^{\nu} / 2
\end{aligned}
$$


while for the contracted next-to-leading twist amplitude we get

$$
\begin{aligned}
& \mathcal{T}_{\text {twist-3 }}^{\mu \nu} q_{1 \nu}=-\sum_{f} Q_{f}^{2} \int_{-1}^{1} d \beta \int_{-1+|\beta|}^{1-|\beta|} d \alpha h_{f}(\beta, \alpha, t) r^{\mu} / 2, \\
& q_{2 \mu} \mathcal{T}_{t w i s t-3}^{\mu \nu}=\sum_{f} Q_{f}^{2} \int_{-1}^{1} d \beta \int_{-1+|\beta|}^{1-|\beta|} d \alpha h_{f}(\beta, \alpha, t) r^{\nu} / 2 .
\end{aligned}
$$

Clearly, even at the level $\mathcal{O}(t / s)^{0}$, the amplitude $\mathcal{T}_{\text {twist-2 }}^{\mu \nu}$ alone is not gauge invariant. However, the electromagnetic gauge invariance at this level can be recovered by including the contribution from $\mathcal{T}_{\text {twist }-3}^{\mu \nu}$. The latter exactly cancels Eq. (323).

\section{VIII.3.4 Helicity Amplitudes}

We shall project the Compton scattering amplitude onto the polarization states $\epsilon_{1 \nu} \equiv$ $\epsilon_{\nu}\left(q_{1}\right)$ and $\epsilon_{2 \mu} \equiv \epsilon_{\mu}\left(q_{2}\right)$ of the physical photons. In the Compton-like processes, these two states can be written as the linear combination of two basic polarization fourvectors [114],

$$
\begin{aligned}
& e_{1}^{\lambda}=\frac{n_{1}^{\lambda}}{\sqrt{-n_{1}^{2}}} \\
& e_{2}^{\lambda}=\frac{n_{2}^{\lambda}}{\sqrt{-n_{2}^{2}}}
\end{aligned}
$$

where

$$
n_{1}^{\lambda}=p^{\lambda}-\frac{(p \cdot q)}{q^{2}} q^{\lambda}
$$

is in the reaction plane and

$$
n_{2}^{\lambda}=\epsilon^{\lambda \mu \nu \rho} p_{\mu} r_{\nu} q_{\rho}
$$

is perpendicular to it. Then, by construction $\left(q \cdot \epsilon_{1}\right)=\left(r \cdot \epsilon_{1}\right)=0$ and similarly for $\epsilon_{2}$. The contracted twist- 2 amplitude is

$$
\begin{aligned}
\mathcal{T}_{t w i s t-2}^{\mu \nu} \epsilon_{2 \mu}^{*} \epsilon_{1 \nu}= & 2 \sum_{f} Q_{f}^{2} \int_{-1}^{1} d \beta \int_{-1+|\beta|}^{1-|\beta|} d \alpha h_{f}(\beta, \alpha, t) \frac{1}{(k+q)^{2}} \\
& \times\left\{2 \beta^{2}\left(p \cdot \epsilon_{1}\right)\left(p \cdot \epsilon_{2}^{*}\right)-\left(\epsilon_{1} \cdot \epsilon_{2}^{*}\right)\left[k^{2}+(k \cdot q)\right]\right\} \\
= & \sum_{f} Q_{f}^{2} \int_{-1}^{1} d \beta \int_{-1+|\beta|}^{1-|\beta|} d \alpha h_{f}(\beta, \alpha, t) \\
& \times\left\{\left[-1+\frac{\left(\beta^{2}-\alpha^{2}-1\right) t / 4}{(k+q)^{2}}\right]\left(\epsilon_{1} \cdot \epsilon_{2}^{*}\right)+\frac{4 \beta^{2}}{(k+q)^{2}}\left(p \cdot \epsilon_{1}\right)\left(p \cdot \epsilon_{2}^{*}\right)\right\}
\end{aligned}
$$


and for the twist-3 part we find

$$
\begin{aligned}
\mathcal{T}_{t w i s t-3}^{\mu \nu} \epsilon_{2 \mu}^{*} \epsilon_{1 \nu}= & -t \sum_{f} Q_{f}^{2} \int_{-1}^{1} d \beta \beta^{2} \int_{-1+|\beta|}^{1-|\beta|} d \alpha h_{f}(\beta, \alpha, t)\left(p \cdot \epsilon_{1}\right)\left(p \cdot \epsilon_{2}^{*}\right) \\
& \times \int_{0}^{1} d v\left\{\frac{\bar{v}-v \alpha}{\left[(k+q)^{2}-\bar{v} k_{1}^{2}\right]^{2}}+\frac{\bar{v}+v \alpha}{\left[(k+q)^{2}-\bar{v} k_{2}^{2}\right]^{2}}\right\}
\end{aligned}
$$

Furthermore, we introduce twist- 2 and twist-3 photon helicity amplitudes by projecting the photon polarizations onto the basic vectors $e_{1}$ and $e_{2}$,

$$
\begin{aligned}
& H_{t w i s t-2(3)}^{(1,1)}=\mathcal{T}_{t w i s t-2(3)}^{\mu \nu} e_{1 \mu}^{*} e_{1 \nu} \\
& H_{t w i s t-2(3)}^{(1,2)}=\mathcal{T}_{t w i s t-2(3)}^{\mu \nu} e_{1 \mu}^{*} e_{2 \nu} \\
& H_{t w i s t-2(3)}^{(2,1)}=\mathcal{T}_{t w i s t-2(3)}^{\mu \nu} e_{2 \mu}^{*} e_{1 \nu} \\
& H_{t w i s t-2(3)}^{(2,2)}=\mathcal{T}_{t w i s t-2(3)}^{\mu \nu} e_{2 \mu}^{*} e_{2 \nu}
\end{aligned}
$$

Out of eight helicity amplitudes, only three are nonzero, namely,

$$
\begin{aligned}
H_{t w i s t-2}^{(1,1)}= & \sum_{f} Q_{f}^{2} \int_{-1}^{1} d \beta \int_{-1+|\beta|}^{1-|\beta|} d \alpha h_{f}(\beta, \alpha, t) \\
& \times\left\{1-\frac{\left(\beta^{2}-\alpha^{2}-1\right)}{(k+q)^{2}} \frac{t}{4}+\frac{4 \beta^{2}}{(k+q)^{2}} \frac{u s}{t}\right\}, \\
H_{t w i s t-2}^{(2,2)}= & \sum_{f} Q_{f}^{2} \int_{-1}^{1} d \beta \int_{-1+|\beta|}^{1-|\beta|} d \alpha h_{f}(\beta, \alpha, t) \\
& \times\left\{1-\frac{\left(\beta^{2}-\alpha^{2}-1\right)}{(k+q)^{2}} \frac{t}{4}\right\}, \\
H_{t w i s t-3}^{(1,1)}= & -u s \sum_{f} Q_{f}^{2} \int_{-1}^{1} d \beta \beta^{2} \int_{-1+|\beta|}^{1-|\beta|} d \alpha h_{f}(\beta, \alpha, t) \\
& \int_{0}^{1} d v\left\{\frac{\bar{v}-v \alpha}{\left[(k+q)^{2}-\bar{v} k_{1}^{2}\right]^{2}}+\frac{\bar{v}+v \alpha}{\left[(k+q)^{2}-\bar{v} k_{2}^{2}\right]^{2}}\right\},
\end{aligned}
$$

where we have used

$$
\begin{aligned}
\left(p \cdot e_{1}\right)^{2} & =-p^{2}+\frac{(p \cdot q)^{2}}{q^{2}} \\
& =\frac{t}{4}-\frac{s^{2}}{t}\left(1+\frac{t}{2 s}\right)^{2} \\
& =\frac{u s}{t}
\end{aligned}
$$




\section{VIII.3.5 Model}

For the pion double distribution $h_{f}(\beta, \alpha, t)$, we use a simple model in a factorized form [115],

$$
h_{f}(\beta, \alpha, t)=\frac{1}{|\beta|} f(\beta) h(\beta, \alpha) \mathcal{F}(\beta, t)
$$

The profile function is chosen to be infinitely narrow,

$$
h(\beta, \alpha)=\delta(\alpha)
$$

Accordingly, the integral over $\alpha$ in the helicity amplitudes (331) is trivial. Next, for the forward distribution we take

$$
f(\beta)=\frac{3}{4} \frac{1-|\beta|}{\sqrt{|\beta|}},
$$

and the $t$-dependence is described by the following Regge ansatz

$$
\mathcal{F}(\beta, t)=|\beta|^{-\alpha^{\prime} t(1-|\beta|)}
$$

where the parameter $\alpha^{\prime}=1 \mathrm{GeV}^{-2}$. The summation of quark flavors in Eq. (331) gives an overall charge factor of $5 / 9$.

We calculate separately the $s$ - and $u$-channel contributions in the helicity amplitudes. Using Eq. (313) and making the change in the variable $\beta \rightarrow-\beta$ in the $u$-channel, we have for the helicity amplitude $H_{t w i s t-2}^{(1,1)}$

$$
\begin{aligned}
H_{\text {twist-2 }}^{(1,1)}= & \frac{5}{9} \int_{0}^{1} d \beta \frac{1}{\beta} \frac{3}{4} \frac{(1-\beta)}{\sqrt{\beta}} \beta^{\alpha^{\prime}|t|(1-\beta)} \\
& \times\left[1+\frac{\left(\beta^{2}-1\right)|t| / 4}{\beta s+(1-\beta)^{2}|t| / 4}-\frac{4 \beta^{2} u s /|t|}{\beta s+(1-\beta)^{2}|t| / 4}\right] \\
& +\frac{5}{9} \int_{0}^{1} d \beta \frac{1}{\beta} \frac{3}{4} \frac{(1-\beta)}{\sqrt{\beta}} \beta^{\alpha^{\prime}|t|(1-\beta)} \\
& \times\left[1+\frac{\left(\beta^{2}-1\right)|t| / 4}{\beta u+(1-\beta)^{2}|t| / 4}-\frac{4 \beta^{2} u s /|t|}{\beta u+(1-\beta)^{2}|t| / 4}\right],
\end{aligned}
$$

and after some manipulation

$$
\begin{aligned}
H_{t w i s t-2}^{(1,1)}= & \frac{5}{9} \frac{3}{2} \int_{0}^{1} d \beta(1-\beta) \beta^{-1 / 2+\alpha^{\prime}|t|(1-\beta)} \\
& \times\left[\frac{\left(2 a^{2}-1\right) \beta+a}{\beta^{2}+2 a \beta+1}+\frac{\left(2 a^{2}-1\right) \beta-a}{\beta^{2}-2 a \beta+1}\right]
\end{aligned}
$$


where the parameter $a=2 s /|t|-1 \geq 1$ depends only on the ratio $s /|t|$. The first term in the square brackets of Eq. (338) corresponds to the $s$-channel contribution. For the moderate invariant momentum transfer, the term has no singularities in the region $0 \leq \beta \leq 1$, in other words, the $s$-channel contribution is real. The situation is, however, different with the second term in the square brackets, which represents the $u$-channel contribution. Its denominator has the roots $\beta_{1,2}=a \pm \sqrt{a^{2}-1}$ (in fact, the denominator is equal to $\left.\beta^{2}-2 a \beta+1+i \epsilon=\left(\beta-\beta_{1}+i \epsilon\right)\left(\beta-\beta_{2}-i \epsilon\right)\right)$ and, unlike the $s$-channel, there is one relevant pole at $\beta=\beta_{2}+i$. The $u$-channel contribution has therefore both the real and imaginary parts. They can be evaluated in the complex plane by making use of the residue theorem in the following way: one introduces a new variable, $\gamma=\beta-1 / 2$, which shifts the integration region from $[0,1]$ to $[-1 / 2,1 / 2]$. Then by closing the contour in the lower half-plane we find

$$
\int_{-1 / 2}^{1 / 2} d \gamma\{\ldots\}=\int_{\pi}^{2 \pi} d \gamma\{\ldots\}
$$

with $\gamma=(1 / 2) \exp (i \phi)$. In summary, the helicity amplitudes $H_{\text {twist-2 }}^{(1,1)}$ has both the real and imaginary parts, where the latter is generated solely by the corresponding $u$-channel contribution. In the similar way, we compute the remaining two helicity amplitudes,

$$
\begin{aligned}
H_{\text {twist }-2}^{(2,2)}= & \frac{5}{9} \frac{3}{2} \int_{0}^{1} d \beta(1-\beta) \beta^{-1 / 2+\alpha^{\prime}|t|(1-\beta)} \\
& \times\left[\frac{\beta+a}{\beta^{2}+2 a \beta+1}+\frac{\beta-a}{\beta^{2}-2 a \beta+1}\right]
\end{aligned}
$$

and

$$
\begin{aligned}
H_{t w i s t-3}^{(1,1)}= & \frac{5}{9} 3\left(a^{2}-1\right) \int_{0}^{1} d \beta(1-\beta) \beta^{1 / 2+\alpha^{\prime}|t|(1-\beta)} \\
& \times\left[\frac{1}{\left(\beta^{2}+2 a \beta+1\right)^{2}}+\frac{1}{\left(\beta^{2}-2 a \beta+1\right)^{2}}\right] .
\end{aligned}
$$

For the sake of simplicity, we have neglected terms $k_{1}^{2}$ and $k_{2}^{2}$ in the denominators of $H_{\text {twist-3 }}^{(1,1)}$, see Eq. (331). Then the integral over $v$ simply gives $1 /(k+q)^{4}$. At present, we only consider the twist-2 helicity amplitudes. In Figs. 50 and 51, their real and imaginary parts are plotted against the center-of-mass scattering angle $\theta_{c m}$ rather than against $-t$ (recall that $t=-s \sin ^{2}\left(\theta_{c m} / 2\right)$ ) in the region of sufficiently large invariant $s=5 \mathrm{GeV}^{2}$. We divide the amplitudes by $5 / 9$ to remove the overall charge factor. Analogous plots are presented in Figs. 52 and 53 for $s=10 \mathrm{GeV}^{2}$, and in Figs. 


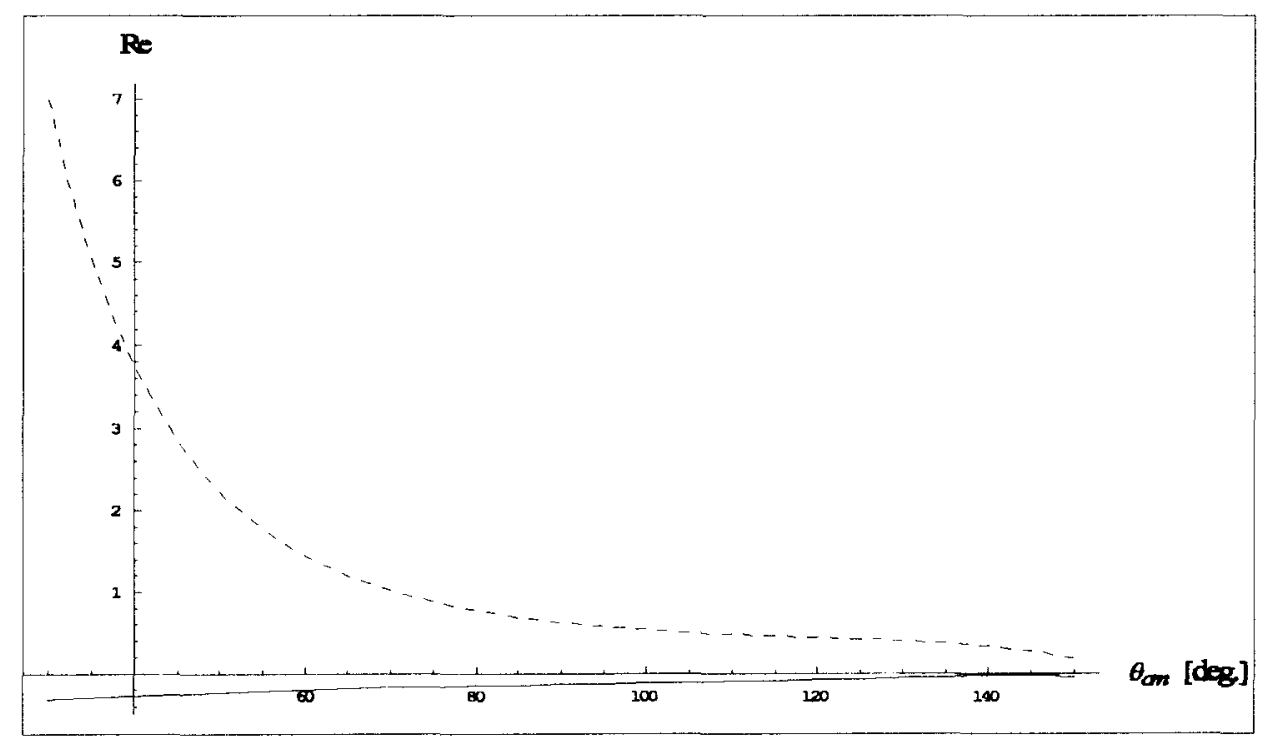

FIG. 50: Real part of the helicity amplitudes $H_{\text {twist-2 }}^{(1,1)}$ (solid line) and $H_{t w i s t-2}^{(2,2)}$ (dashed line) plotted as a function of the center-of-mass scattering angle $\theta_{c m}$ for the invariant $s=5 \mathrm{GeV}^{2}$. The amplitudes are divided by the charge factor $5 / 9$.

54 and 55 for $s=20 \mathrm{GeV}^{2}$. Due to the presence of the term $4 \beta^{2}(u s / t) /(k+q)^{2}$ in the amplitude $H_{\text {twist-2 }}^{(1,1)}$, one might naively expect that the real part of $H_{\text {twist-2 }}^{(1,1)}$ would dominate over the real part of $H_{t w i s t-2}^{(2,2)}$. Both the $s$-channel and the real part of the $u$-channel contributions in $H_{\text {twist-2 }}^{(1,1)}$ are indeed large, however, they come with the opposite signs, and as a result, the real part of the amplitude $H_{t w i s t-2}^{(1,1)}$ diminishes, as oppose to the amplitude $H_{\text {twist-2 }}^{(2,2)}$, in which both terms add up. Another observation is that the imaginary parts of the helicity amplitudes are suppressed with respect to their real parts.

We can now estimate the polarized differential cross sections in our simple model for the pion $\mathrm{DD} h_{f}(\beta, \alpha, t)$. In the center-of-mass frame, we write

$$
\begin{aligned}
d \sigma^{\left(\lambda_{1}, \lambda_{2}\right)}\left(\gamma \pi^{+} \rightarrow \gamma \pi^{+}\right)= & \frac{1}{2 E_{1} 2 \omega_{1} v_{\text {rel }}}(2 \pi)^{4} \delta^{(4)}\left(p_{1}+q_{1}-p_{2}-q_{2}\right) \\
& \times \frac{d \vec{p}_{2}}{(2 \pi)^{3} 2 E_{2}} \frac{d \vec{q}_{2}}{(2 \pi)^{3} 2 \omega_{2}}\left|e^{2} H^{\left(\lambda_{1}, \lambda_{2}\right)}\right|^{2} .
\end{aligned}
$$

Note that we multiplied the helicity amplitude with $e^{2}$, which has been conveniently skipped in our analysis. Partially evaluating the integrals over the final-state momenta, e.g. integration over $\vec{q}_{2}$ kills $\delta^{(3)}\left(\vec{p}_{1}+\vec{q}_{1}-\vec{p}_{2}-\vec{q}_{2}\right)$ and sets $\vec{q}_{2}=-\vec{p}_{2}$, yields 


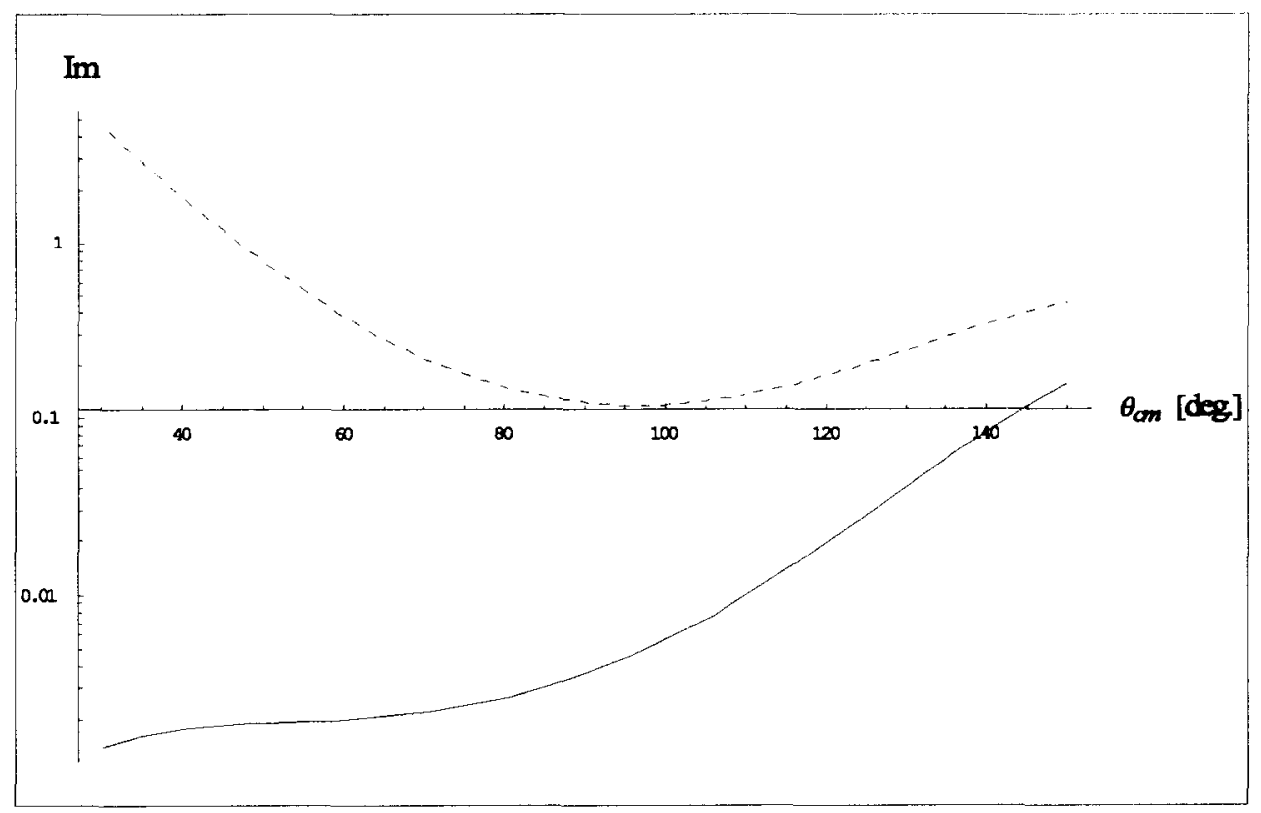

FIG. 51: Imaginary part of the helicity amplitudes $H_{\text {twist-2 }}^{(1,1)}$ (solid line) and $H_{t w i s t-2}^{(2,2)}$ (dashed line) plotted as a function of the center-of-mass scattering angle $\theta_{c m}$ for the invariant $s=5 \mathrm{GeV}^{2}$. The amplitudes are divided by the charge factor $5 / 9$.

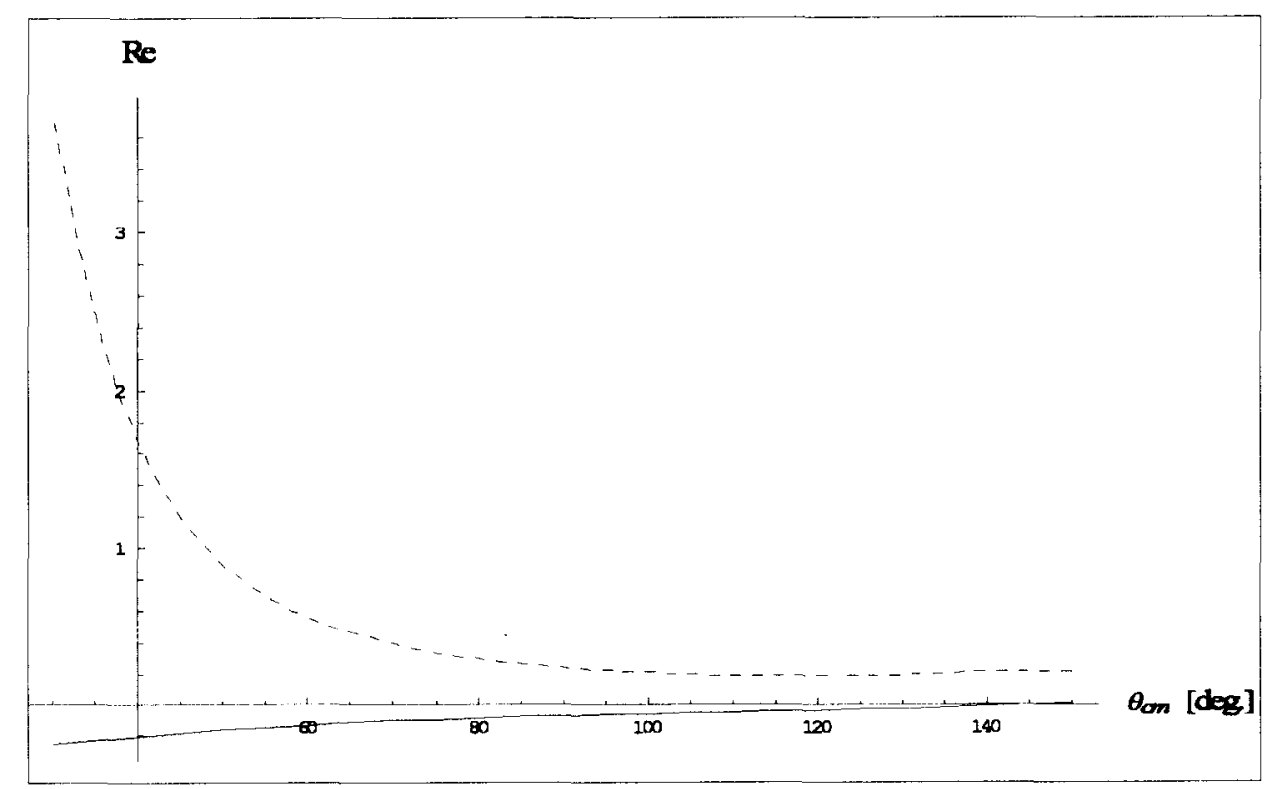

FIG. 52: Real part of the helicity amplitudes $H_{\text {twist-2 }}^{(1,1)}$ (solid line) and $H_{\text {twist-2 }}^{(2,2)}$ (dashed line) plotted as a function of the center-of-mass scattering angle $\theta_{\mathrm{cm}}$ for the invariant $s=10 \mathrm{GeV}^{2}$. The amplitudes are divided by the charge factor $5 / 9$. 


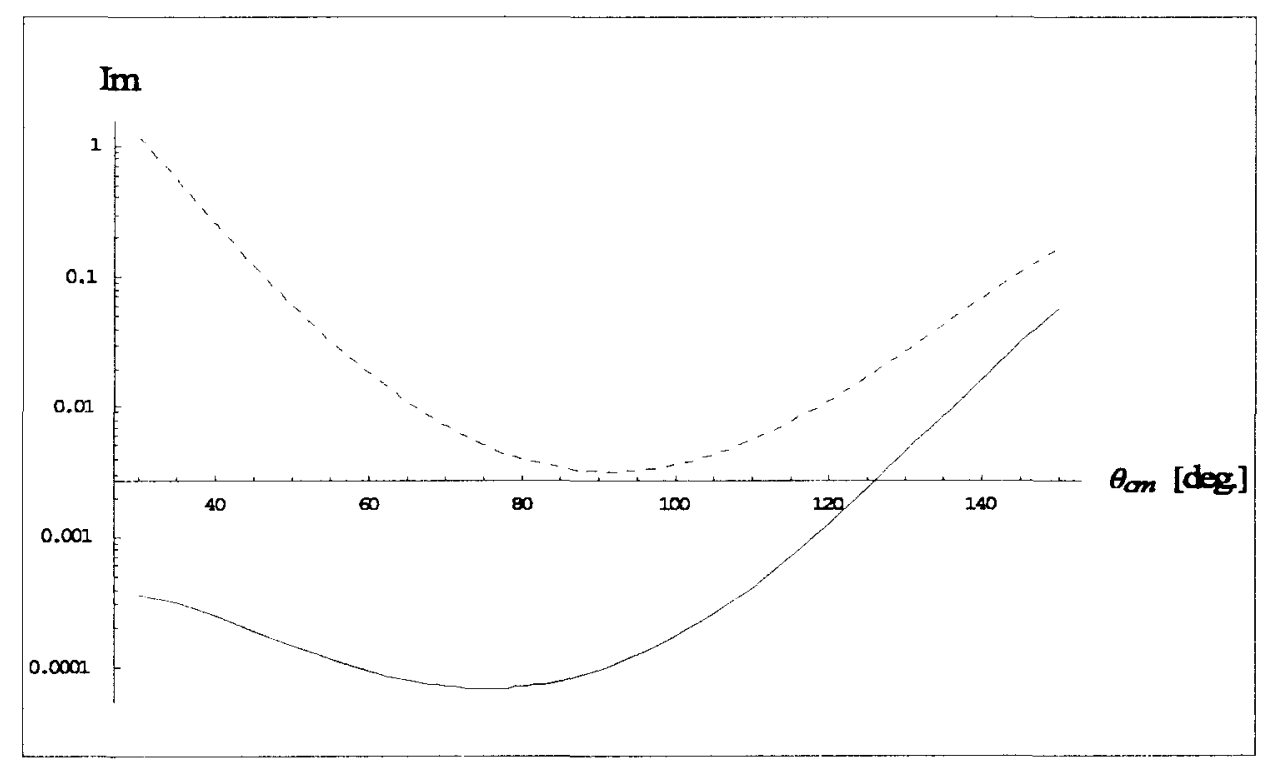

FIG. 53: Imaginary part of the helicity amplitudes $H_{t w i s t-2}^{(1,1)}$ (solid line) and $H_{t w i s t-2}^{(2,2)}$ (dashed line) plotted as a function of the center-of-mass scattering angle $\theta_{\mathrm{cm}}$ for the invariant $s=10 \mathrm{GeV}^{2}$. The amplitudes are divided by the charge factor $5 / 9$.

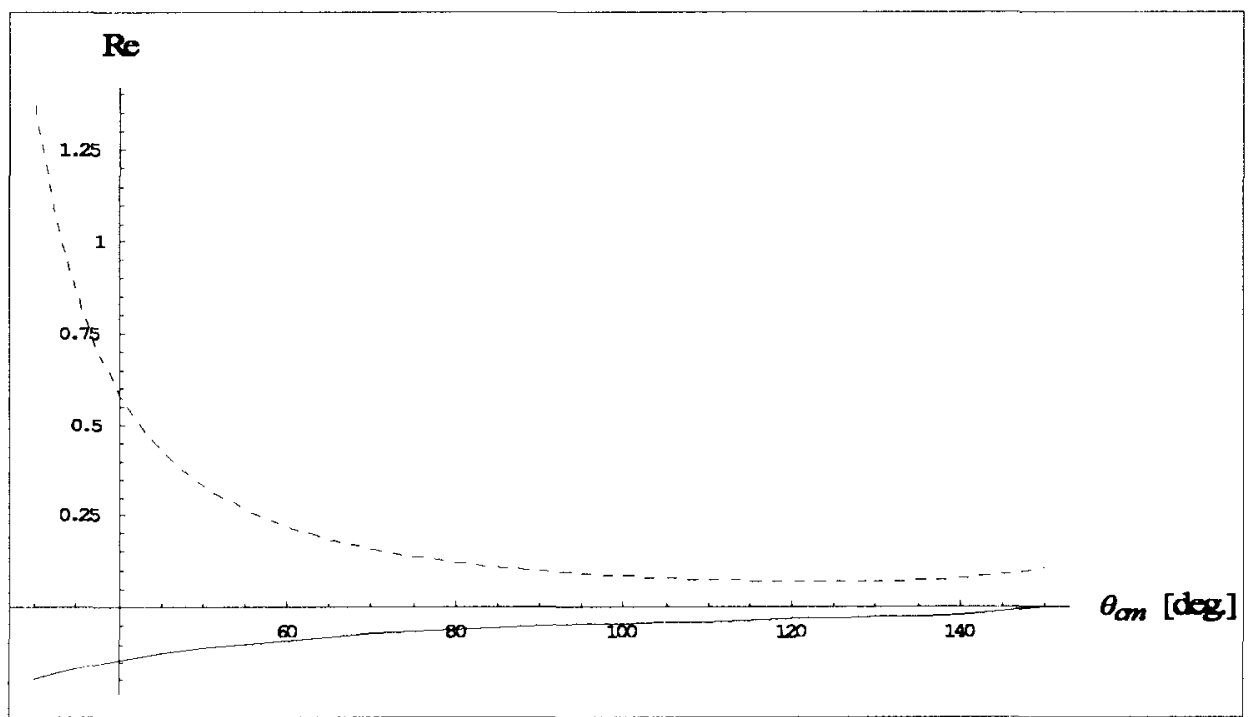

FIG. 54: Real part of the helicity amplitudes $H_{\text {twist }-2}^{(1,1)}$ (solid line) and $H_{\text {twist }-2}^{(2,2)}$ (dashed line) plotted as a function of the center-of-mass scattering angle $\theta_{c m}$ for the invariant $s=20 \mathrm{GeV}^{2}$. The amplitudes are divided by the charge factor $5 / 9$. 


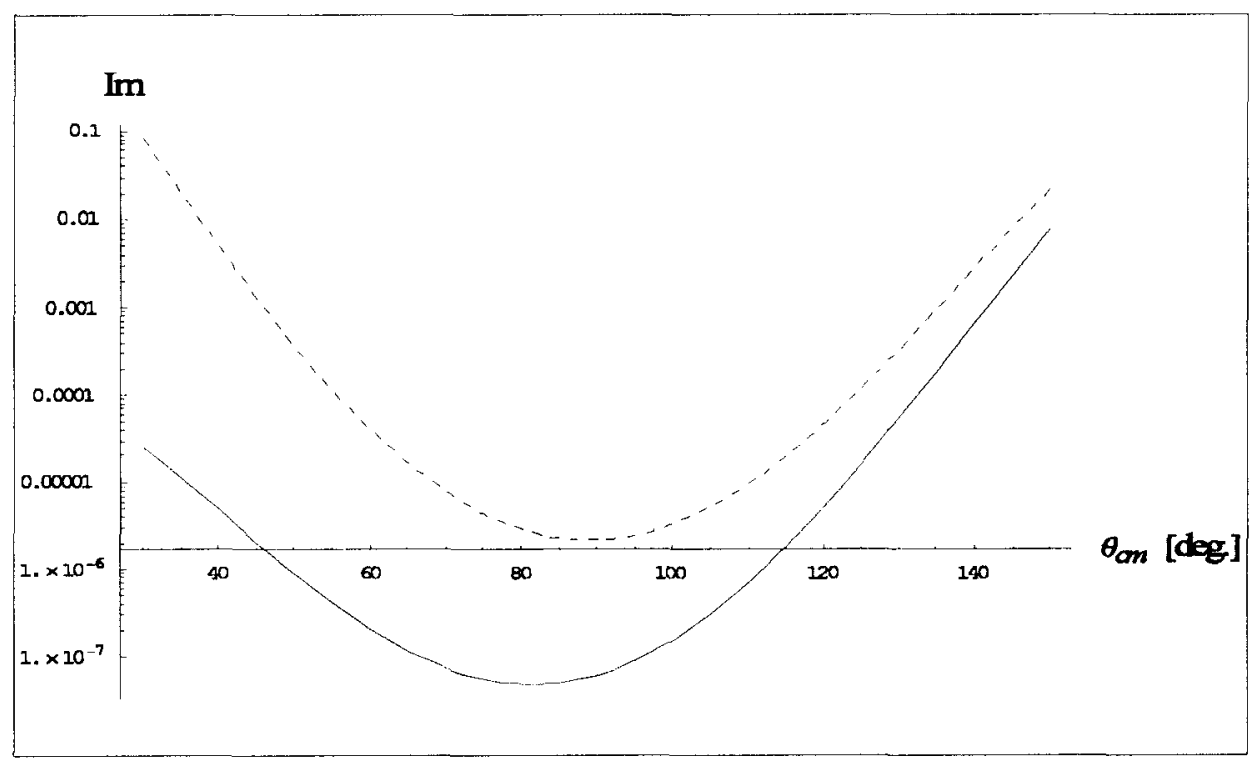

FIG. 55: Imaginary part of the helicity amplitudes $H_{\text {twist-2 }}^{(1,1)}$ (solid line) and $H_{\text {twist-2 }}^{(2,2)}$ (dashed line) plotted as a function of the center-of-mass scattering angle $\theta_{c m}$ for the invariant $s=20 \mathrm{GeV}^{2}$. The amplitudes are divided by the charge factor $5 / 9$.

at high energies (and thus $v_{\text {rel }}=2$ )

$$
d \sigma^{\left(\lambda_{1}, \lambda_{2}\right)}\left(\gamma \pi^{+} \rightarrow \gamma \pi^{+}\right)=\frac{1}{2 s} \frac{1}{4} \frac{1}{(2 \pi)^{2}} \delta\left(2 E_{1}-2 E_{2}\right) d E_{2} d \Omega\left|e^{2} H^{\left(\lambda_{1}, \lambda_{2}\right)}\right|^{2},
$$

where $s=E_{\mathrm{cm}}^{2}$ and $E_{1}=E_{\mathrm{cm}} / 2$. The differential cross section can be further simplified into

$$
\frac{d^{2} \sigma^{\left(\lambda_{1}, \lambda_{2}\right)}\left(\gamma \pi^{+} \rightarrow \gamma \pi^{+}\right)}{d \Omega}=\frac{e^{4}}{64 \pi^{2} s}\left|H^{\left(\lambda_{1}, \lambda_{2}\right)}\right|^{2},
$$

which can be written in the invariant form as

$$
\frac{d \sigma^{\left(\lambda_{1}, \lambda_{2}\right)}}{d t}=\frac{e^{4}}{16 \pi s^{2}}\left|H^{\left(\lambda_{1}, \lambda_{2}\right)}\right|^{2} .
$$

The $\theta_{c m}$-dependence of the twist-2 cross sections $\sigma^{(1,1)}$ and $\sigma^{(2,2)}$ are illustrated on a logarithmic scale for all three values of the invariant $s$ in Figs. 56 and 57, respectively. Similarly to the situation with the nucleon target (see, e.g. [38]), the plots reveal a slight increase in both polarized cross sections at large scattering angles, $\theta_{c m} \geq 130^{\circ}$. Moreover, by comparing their orders of magnitude, we find that the cross section $\sigma^{(1,1)}$ is significantly suppressed compared to $\sigma^{(2,2)}$. Next, the angular dependence of the combination $s^{4.6} d \sigma^{(2,2)} / d t$ is shown in Fig. 58. The curves obey scaling behavior, i.e. they basically coincide up to $\theta_{c m} \simeq 100^{\circ}$. 


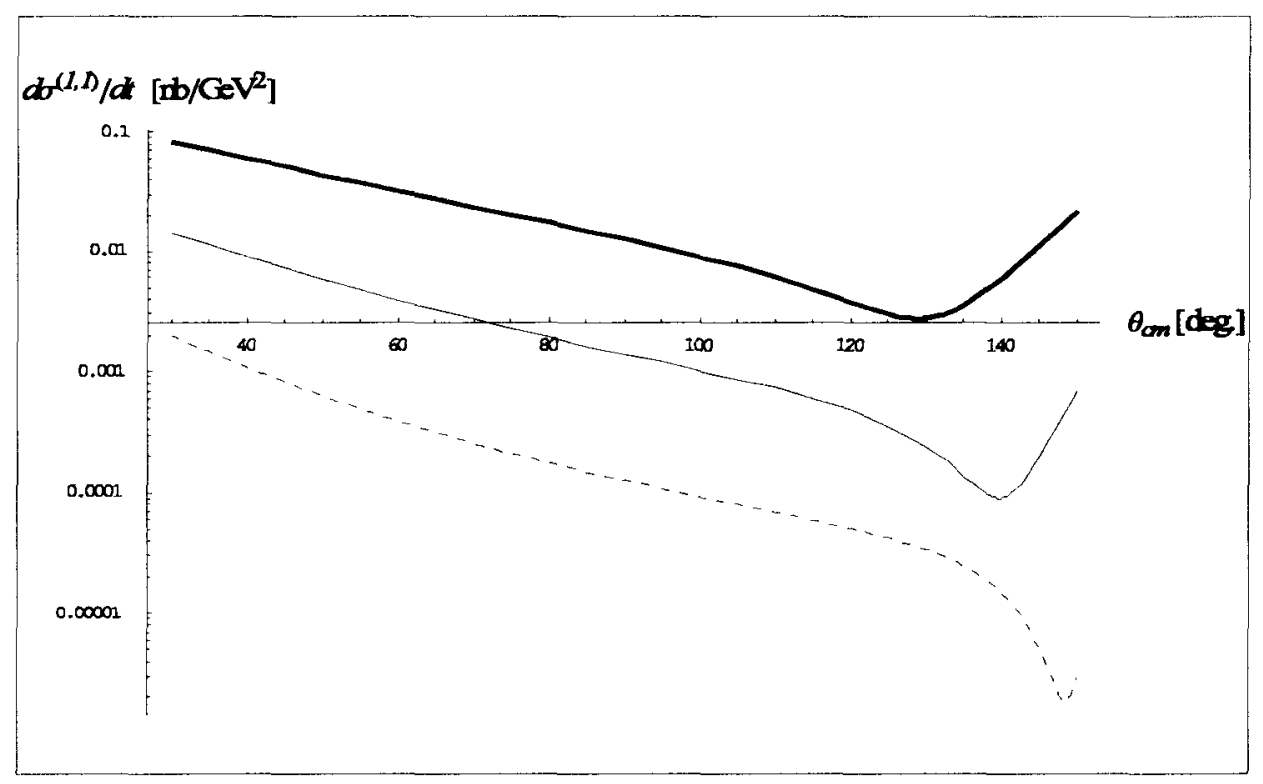

FIG. 56: Twist-2 cross section $\sigma^{(1,1)}$ plotted as a function of the center-of-mass scattering angle $\theta_{c m}$ for the invariant $s=5 \mathrm{GeV}^{2}$ (bold solid line), $s=10 \mathrm{GeV}^{2}$ (solid line) and $s=20 \mathrm{GeV}^{2}$ (dashed line).

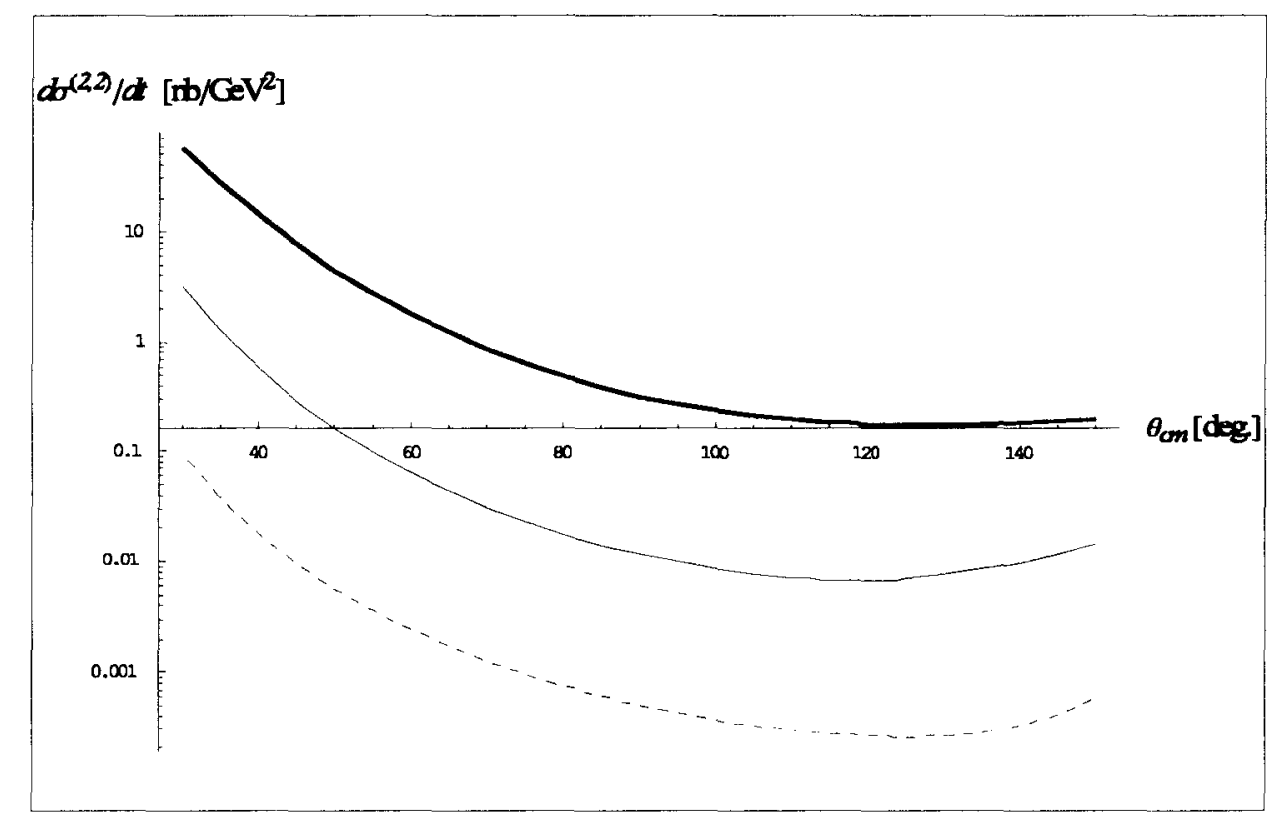

FIG. 57: Twist-2 cross section $\sigma^{(2,2)}$ plotted as a function of the center-of-mass scattering angle $\theta_{c m}$ for the invariant $s=5 \mathrm{GeV}^{2}$ (bold solid line), $s=10 \mathrm{GeV}^{2}$ (solid line) and $s=20 \mathrm{GeV}^{2}$ (dashed line). 


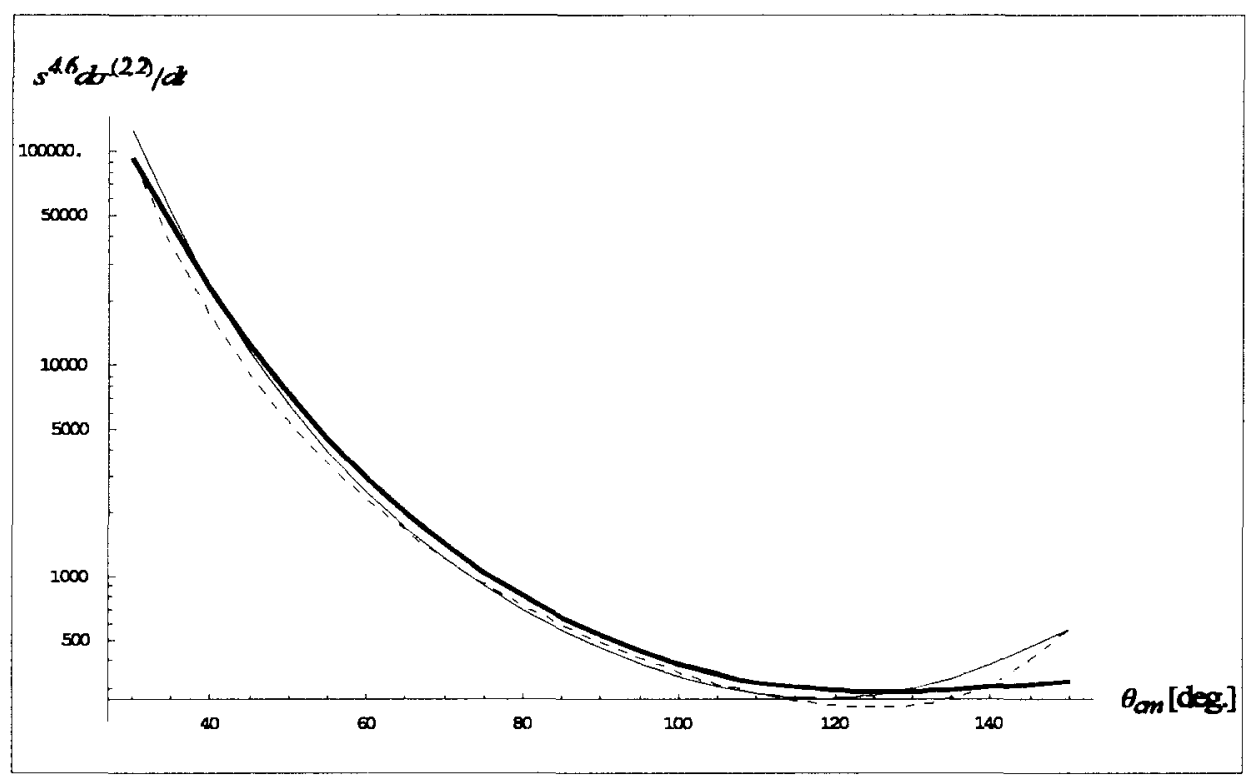

FIG. 58: Combination $s^{4.6} d \sigma^{(2,2)} / d t$ plotted as a function of the center-of-mass scattering angle $\theta_{c m}$ for the invariant $s=5 \mathrm{GeV}^{2}$ (bold solid line), $s=10 \mathrm{GeV}^{2}$ (solid line) and $s=20 \mathrm{GeV}^{2}$ (dashed line).

\section{VIII.4 COMPTON SCATTERING ON A PION IN QED}

We compare the QCD results of Section VIII.3 with the QED calculation. Assuming that $\pi^{+}$is an elementary particle we have, in the lowest order in $\alpha_{E M}$, three relevant Feynman diagrams, see Fig. 59, namely, the $s$ - and $u$-channel tree diagrams and the four-point contact interaction diagram. Adding them coherently, one finds for the T-matrix

$$
\begin{aligned}
i \mathrm{~T}= & {\left[i|e|\left(2 p_{2}+q_{2}\right)^{\mu} \frac{i}{\left(p_{1}+q_{1}\right)^{2}-m_{\pi}^{2}} i|e|\left(2 p_{1}+q_{1}\right)^{\nu}\right.} \\
& +i|e|\left(2 p_{2}-q_{1}\right)^{\nu} \frac{i}{\left(p_{2}-q_{1}\right)^{2}-m_{\pi}^{2}} i|e|\left(2 p_{1}-q_{2}\right)^{\mu} \\
& \left.+2 i|e|^{2} g^{\mu \nu}\right] \epsilon_{1 \nu} \epsilon_{2 \mu}^{*} .
\end{aligned}
$$

The expression in the brackets corresponds to the Compton scattering amplitude $\mathcal{T}^{\mu \nu}$ in QED. We can immediately check its gauge invariance and find

$$
\begin{aligned}
\mathcal{T}^{\mu \nu} q_{1 \nu} & =-i e^{2}\left\{\frac{\left(2 p_{2}+q_{2}\right)^{\mu}\left[2\left(p_{1} \cdot q_{1}\right)+q_{1}^{2}\right]}{2\left(p_{1} \cdot q_{1}\right)}+\frac{\left(2 p_{1}-q_{2}\right)^{\mu}\left[2\left(p_{2} \cdot q_{1}\right)-q_{1}^{2}\right]}{-2\left(p_{1} \cdot q_{1}\right)}-2 q_{1}^{\mu}\right\} \\
& =-2 i e^{2}\left(p_{2}+q_{2}-p_{1}-q_{1}\right)^{\mu} \\
& =0 .
\end{aligned}
$$



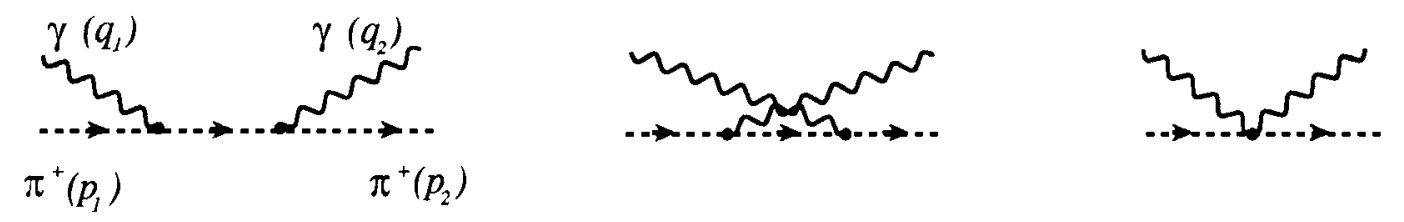

FIG. 59: Feynman diagrams for Compton scattering on a pion in QED.

Similarly, we prove that $q_{2 \mu} \mathcal{T}^{\mu \nu}=0$.

Neglecting now the pion mass in Eq. (346) (recall that $s,-t,-u \gg m_{\pi}^{2}$ and hence $s+t+u=2 m_{\pi}^{2} \simeq 0$ ), we get

$$
\mathrm{T}=-2 e^{2}\left[\frac{2\left(p_{1} \cdot \epsilon_{1}\right)\left(p_{2} \cdot \epsilon_{2}^{*}\right)}{s}+\frac{2\left(p_{1} \cdot \epsilon_{2}^{*}\right)\left(p_{2} \cdot \epsilon_{1}\right)}{u}-\left(\epsilon_{1} \cdot \epsilon_{2}^{*}\right)\right] .
$$

By writing the initial and final pion momenta as $p_{1}=p+r / 2$ and $p_{2}=p-r / 2$, respectively, and imposing $\left(r \cdot \epsilon_{1}\right)=\left(r \cdot \epsilon_{2}^{*}\right)=0$, we can express the T-matrix in terms of the average pion momentum,

$$
\mathrm{T}=-2 e^{2}\left[2\left(\frac{1}{s}+\frac{1}{u}\right)\left(p \cdot \epsilon_{1}\right)\left(p \cdot \epsilon_{2}^{*}\right)-\left(\epsilon_{1} \cdot \epsilon_{2}^{*}\right)\right] .
$$

Finally, the projection of polarization vectors $\epsilon_{1}$ and $\epsilon_{2}$ onto $e_{1}$ and $e_{2}$ gives the following photon helicity amplitudes in QED

$$
\begin{aligned}
H^{(1,1)} & =-2 e^{2}\left[2\left(\frac{1}{s}+\frac{1}{u}\right) \frac{u s}{t}+1\right] \\
& =2 e^{2} \\
H^{(1,2)} & =0 \\
H^{(2,1)} & =0 \\
H^{(2,2)} & =-2 e^{2} .
\end{aligned}
$$

The QED helicity amplitudes $H^{(1,1)}$ and $H^{(2,2)}$ are opposite in sign but constant. By comparing them to the real parts of the QCD helicity amplitudes $H_{\text {twist-2 }}^{(1,1)}$ and $H_{t w i s t-2}^{(2,2)}$ (and, as usual, ignoring $-e^{2}$ ), we can establish the effective form factor, which solely describes the underlying QCD dynamics.

In summary, we presented an improved treatment of the DD formalism to the case with the pion target. In addition to deriving the amplitude in the twist-3 approximation, the helicity properties were considered. We gave predictions for the polarized cross sections with the use of the simple model for the pion double distribution. 


\section{CHAPTER IX}

\section{CONCLUSIONS AND OUTLOOK}

In this dissertation we have studied inclusive and exclusive Compton processes in quantum chromodynamics. Let us summarize our main conclusions.

First, we briefly reviewed the crown example of the hard (light-cone dominated) inclusive scattering process in QCD known as deeply inelastic lepton-nucleon scattering. We introduced the forward virtual Compton scattering amplitude and discussed its relation to DIS. We presented the cross sections results in terms of structure functions for both the electromagnetic and weak probes. The last part of Chapter II was devoted to the QCD parton model. This simple model, which naturally emerges from the QCD operator product expansion, successfully describes the scaling property of structure functions, and further allows to express them in terms of the parton distributions functions.

In Chapter III, we started with the systematic description of old phenomenological functions (form factors, parton distribution functions and distribution amplitudes) that were used for years in the studies of hadrons. New phenomenological functions (generalized parton distributions) became necessary in order to generalize the Compton scattering amplitude in the region of nonforward (skewed) kinematics. They were introduced in various ways and under different names as nonperturbative functions describing the soft (long-distance) part of the factorized scattering amplitudes in QCD. Generalized parton distributions accumulate the most complete information about the hadronic structure, and as such combine the features of form factors, usual parton distributions and distribution amplitudes. We presented some of their theoretical aspects.

Generalized parton distributions have numerous applications to hard processes, in particular, to the exclusive ones. The pedagogical example in this respect is deeply virtual Compton scattering. In Chapter IV, we demonstrated the derivation of the amplitude at the leading twist-2 level in the DVCS kinematics, using the light-cone expansion in terms of QCD string operators in coordinate space. We investigated the electromagnetic gauge invariance of the amplitude. The latter is violated with respect to the initial virtual photon and therefore, higher twist corrections have to be taken into account. Furthermore, we discussed the kinematics and introduced a 
toy model for the nucleon off-forward parton distributions, which did not include the contribution from the sea quarks. We estimated the unpolarized cross sections in the kinematical region relevant to Jefferson Lab, and observed that unfortunately the contamination from the Bethe-Heitler process predominated the pure DVCS signal. The way out is to exploit the interference between the two processes. This approach allows to project out independently both the real and imaginary parts of the DVCS amplitude and probe different linear combinations of GPDs.

In Chapter V, we studied the inclusive photoproduction of massive lepton pairs. The process was considered in the framework of the parton model at rather high photon beam energy. Again one deals with two types of subprocesses. We picked only the lowest order electromagnetic contributions and calculated both the Compton and Bethe cross sections. We found that in the forward direction the Compton contribution was slightly larger than the Bethe-Heitler contribution, however, it decreased much faster. In addition, we illustrated that the interference terms cancel in pairs, after being integrated over the momenta of final leptons.

The inverse process to DVCS is known as time-like Compton scattering. It can be accessed through the photoproduction of a heavy lepton pair. In Chapter VI, we considered this particular reaction in the DVCS kinematics. The unpolarized cross section results revealed that the Compton contribution was significantly suppressed with respect to the Bethe-Heitler contribution. In analogy with the DVCS process, we expect that the interference terms between the Compton and Bethe-Heitler processes, in particular, from the experiments with the polarized photon beam, may play an important role in extracting new information on GPDs. Thus more future studies are needed in this direction.

By utilizing the weak currents to probe the nucleon structure we are able to measure, due to the $V-A$ nature of interaction, a different combination of generalized parton distributions as well as the distributions that are nondiagonal in quark flavor. Hence neutrino-induced virtual Compton scattering, which has been studied in Chapter VII, provides an important tool to complement the study of GPDs in more familiar electron-induced DVCS or exclusive meson production processes. We derived the twist-2 amplitudes for the weak neutral and weak charged current interactions, and gave predictions for the cross sections in the kinematics relevant to future high-intensity neutrino experiments. At small scattering angles, we observed that, unlike the standard electromagnetic DVCS process, the Compton contribution 
was somewhat comparable to the corresponding Bethe-Heitler contribution in the weak neutral interaction sector. Nevertheless, the Compton signal was well above the Bethe-Heitler one in the weak charged interaction sector. From the theoretical side, it is expected to use a more realistic model for the nucleon GPDs, and further check separately the contributions from the plus and minus distributions, in particularly, the contribution coming from the pion pole, i.e. the $\tilde{E}_{f}$ distribution. Moreover, one may estimate the contribution from the interference of the Compton with the Bethe-Heitler process, and extend the approach in order to include the twist-3 terms.

In the final chapter, the GPD formalism was applied to real Compton scattering. Again we used the same light-cone expansion technique as in Chapters IV and VII, however, in addition to the twist-2 operators we have also included a set of kinematical twist-3 operators. They appear as total derivatives of twist-2 operators. Unlike the previous chapters, we considered, for simplicity, the pion target. Instead of off-forward parton distributions, more general objects, namely, the double distributions were used to describe the nonperturbative stages of interaction. We obtained the expressions for the twist-2 and twist-3 Compton scattering amplitudes. It was shown, in the lowest order in the invariant momentum transfer, that the kinematical twist-3 contribution to the amplitude for pion was required to restore the tranversality. Next we found that a divergent part of the twist-3 amplitude had zero projection on the polarization vectors of initial and final photons, and calculated the photon helicity amplitudes, using a simple model for the pion double distribution. They were compared to QED helicity amplitudes. Finally, we estimated the relevant polarized cross sections, observed their approximate scaling behavior, and established the noncanonical powers. A straightforward generalization to the case with the nucleon target as well as the study of the exclusive production of two pions in two-photon collisions (the latter is a crossing process to Compton scattering on a pion) are in progress. 


\section{BIBLIOGRAPHY}

[1] G. Zweig, Preprints CERN-TH 401 and 412 (1964).

[2] M. Gell-Mann, Phys. Lett. 8, 214 (1964).

[3] J. D. Bjorken and E. A. Paschos, Phys. Rev. 185, 1975 (1969).

[4] R. P. Feynman, Phys. Rev. Lett. 23, 1415 (1969).

[5] R. P. Feynman, Photon-Hadron Interactions, Reading, USA: W. A. Benjamin (1972).

[6] C. N. Yang and R. L. Mills, Phys. Rev. 96, 191 (1954).

[7] D. J. Gross and F. Wilczek, Phys. Rev. Lett. 30, 1343 (1973).

[8] H. D. Politzer, Phys. Rev. Lett. 30, 1346 (1973).

[9] H. D. Politzer, Phys. Rept. 14, 129 (1974).

[10] N. N. Bogolubov, B. V. Struminsky and A. N. Tavkhelidze, Preprint JINR D-1968, Dubna (1965).

[11] M. Y. Han and Y. Nambu, Phys. Rev. 139, B1006 (1965).

[12] Y. Miyamoto, Prog. Theor. Phys. Suppl. Extra Number (1965), 187.

[13] H. Fritzsch and M. Gell-Mann, in: Proceedings of the XVI International Conference on High Energy Physics, edited by J. D. Jackson and A. Roberts, Fermilab, 1972 Vol. 2, p.135, hep-ph/0208010.

[14] H. Fritzsch, M. Gell-Mann and H. Leutwyler, Phys. Lett. B 47, 365 (1973).

[15] D. Amati, R. Petronzio and G. Veneziano, Nucl. Phys. B 140, 54 (1978).

[16] D. Amati, R. Petronzio and G. Veneziano, Nucl. Phys. B 146, 29 (1978).

[17] S. B. Libby and G. Sterman, Phys. Rev. D 18, 3252 (1978).

[18] A. V. Efremov and A. V. Radyushkin, Theor. Math. Phys. 44, 573 (1980).

[19] A. V. Efremov and A. V. Radyushkin, Theor. Math. Phys. 44, 664 (1981). 
[20] A. V. Efremov and A. V. Radyushkin, Theor. Math. Phys. 44, 774 (1981)

[21] R. K. Ellis, H. Georgi, M. Machacek, H. D. Politzer and G. G. Ross, Nucl. Phys. B 152, 285 (1979).

[22] A. H. Mueller, Phys. Rev. D 18, 3705 (1978).

[23] K. G. Wilson, Phys. Rev. 179, 1499 (1969).

[24] R. A. Brandt and G. Preparata, Nucl. Phys. B 27, 541 (1972).

[25] D. J. Gross and F. Wilczek, Phys. Rev. D 8, 3633 (1973).

[26] D. J. Gross and F. Wilczek, Phys. Rev. D 9, 980 (1974).

[27] H. Georgi and H. D. Politzer, Phys. Rev. D 9, 416 (1974).

[28] M. Lazar, Group Theoretical Analysis of Light-Cone Dominated Hadronic Processes and Twist Decomposition of Nonlocal Operators in Quantum Chromodynamics, Ph.D. Thesis (2002), hep-ph/0308049.

[29] D. Muller, D. Robaschik, B. Geyer, F. M. Dittes and J. Horejsi, Fortsch. Phys. 42, 101 (1994), hep-ph/9812448.

[30] X. D. Ji, Phys. Rev. Lett. 78, 610 (1997), hep-ph/9603249.

[31] X. D. Ji, Phys. Rev. D 55, 7114 (1997), hep-ph/9609381.

[32] A. V. Radyushkin, Phys. Lett. B 380, 417 (1996), hep-ph/9604317.

[33] A. V. Radyushkin, Phys. Lett. B 385, 333 (1996), hep-ph/9605431.

[34] A. V. Radyushkin, Phys. Rev. D 56, 5524 (1997), hep-ph/9704207.

[35] M. Vanderhaeghen, P. A. M. Guichon and M. Guidal, Phys. Rev. D 60, 094017 (1999), hep-ph/9905372.

[36] K. Goeke, M. V. Polyakov and M. Vanderhaeghen, Prog. Part. Nucl. Phys. 47, 401 (2001), hep-ph/0106012.

[37] J. C. Collins, L. Frankfurt and M. Strikman, Phys. Rev. D 56, 2982 (1997), hep-ph/9611433. 
[38] A. V. Radyushkin, Phys. Rev. D 58, 114008 (1998), hep-ph/9803316.

[39] M. Diehl, T. Feldmann, R. Jakob and P. Kroll, Eur. Phys. J. C 8, 409 (1999), hep-ph/9811253.

[40] M. Diehl, T. Feldmann, R. Jakob and P. Kroll, Phys. Lett. B 460, 204 (1999), hep-ph/9903268.

[41] W. Greiner and A. Schafer, Quantum Chromodynamics, Berlin, Germany: Springer-Verlag (1994).

[42] A. W. Thomas and W. Weise, The Structure of the Nucleon, Berlin, Germany: Wiley-VCH (2001).

[43] R. L. Jaffe, hep-ph/9602236.

[44] J. D. Bjorken, Phys. Rev. 179, 1547 (1969).

[45] E. D. Bloom et al., Phys. Rev. Lett. 23, 930 (1969).

[46] M. Breidenbach et al., Phys. Rev. Lett. 23, 935 (1969).

[47] J. I. Friedman and H. W. Kendall, Ann. Rev. Nucl. Part. Sci. 22, 203 (1972).

[48] G. Sterman, hep-ph/9606312.

[49] M. E. Peskin and D. V. Schroeder, An Introduction to Quantum Field Theory, Reading, USA: Addison-Wesley (1995).

[50] C. G. Callan and D. J. Gross, Phys. Rev. Lett. 22, 156 (1969).

[51] T. Muta, Foundations Of Quantum Chromodynamics: An Introduction to Perturbative Methods in Gauge Theories, World Sci. Lect. Notes Phys. 5, 1 (1987).

[52] A. V. Radyushkin, Nucl. Phys. A 711, 99 (2002).

[53] A. V. Radyushkin, hep-ph/0409215.

[54] A. V. Radyushkin, hep-ph/0101225.

[55] M. Diehl, Phys. Rept. 388, 41 (2003), hep-ph/0307382. 
[56] A. V. Belitsky and A. V. Radyushkin, hep-ph/0504030.

[57] V. N. Gribov and L. N. Lipatov, Sov. J. Nucl. Phys. 15, 438 (1972) [Yad. Fiz. 15, 781 (1972)].

[58] G. Altarelli and G. Parisi, Nucl. Phys. B 126, 298 (1977).

[59] Y. L. Dokshitzer, Sov. Phys. JETP 46, 641 (1977) [Zh. Eksp. Teor. Fiz. 73, $1216(1977)]$.

[60] A. V. Radyushkin, hep-ph/0410276.

[61] A. V. Efremov and A. V. Radyushkin, Theor. Math. Phys. 42, 97 (1980)

[62] A. V. Efremov and A. V. Radyushkin, Phys. Lett. B 94, 245 (1980).

[63] G. P. Lepage and S. J. Brodsky, Phys. Lett. B 87, 359 (1979).

[64] G. P. Lepage and S. J. Brodsky, Phys. Rev. D 22, 2157 (1980).

[65] I. V. Musatov and A. V. Radyushkin, Phys. Rev. D 56, 2713 (1997), hep$\mathrm{ph} / 9702443$.

[66] M. Burkardt, Phys. Rev. D 62, 071503 (2000) [Erratum-ibid. D 66, 119903 (2002)], hep-ph/0005108.

[67] M. Burkardt, Int. J. Mod. Phys. A 18, 173 (2003), hep-ph/0207047.

[68] X. D. Ji and J. Osborne, Phys. Rev. D 58, 094018 (1998), hep-ph/9801260.

[69] J. C. Collins and A. Freund, Phys. Rev. D 59, 074009 (1999), hep$\mathrm{ph} / 9801262$.

[70] B. Lampe and E. Reya, Phys. Rept. 332, 1 (2000), hep-ph/9810270.

[71] B. W. Filippone and X. D. Ji, Adv. Nucl. Phys. 26, 1 (2001), hepph/0101224.

[72] S. D. Bass, hep-ph/0411005.

[73] X. D. Ji, W. Melnitchouk and X. Song, Phys. Rev. D 56, 5511 (1997) hep$\mathrm{ph} / 9702379$. 
[74] V. Y. Petrov, P. V. Pobylitsa, M. V. Polyakov, I. Bornig, K. Goeke and C. Weiss, Phys. Rev. D 57, 4325 (1998), hep-ph/9710270.

[75] A. V. Radyushkin, Phys. Rev. D 59, 014030 (1999), hep-ph/9805342.

[76] L. Mankiewicz, G. Piller and T. Weigl, Eur. Phys. J. C 5, 119 (1998), hep$\mathrm{ph} / 9711227$.

[77] I. V. Musatov and A. V. Radyushkin, Phys. Rev. D 61, 074027 (2000), hep$\mathrm{ph} / 9905376$.

[78] I. I. Balitsky and V. M. Braun, Nucl. Phys. B 311, 541 (1989).

[79] I. V. Anikin, B. Pire and O. V. Teryaev, Phys. Rev. D 62, 071501 (2000) hep-ph/0003203.

[80] M. Penttinen, M. V. Polyakov, A. G. Shuvaev and M. Strikman, Phys. Lett. B 491, 96 (2000), hep-ph/0006321.

[81] A. V. Belitsky and D. Muller, Nucl. Phys. B 589, 611 (2000), hep$\mathrm{ph} / 0007031$.

[82] A. V. Radyushkin and C. Weiss, Phys. Lett. B 493, 332 (2000), hep$\mathrm{ph} / 0008214$.

[83] A. V. Radyushkin and C. Weiss, Phys. Rev. D 63, 114012 (2001), hep$\mathrm{ph} / 0010296$.

[84] N. Kivel and M. V. Polyakov, Nucl. Phys. B 600, 334 (2001), hep$\mathrm{ph} / 0010150$.

[85] N. Kivel, M. V. Polyakov and M. Vanderhaeghen, Phys. Rev. D 63, 114014 (2001), hep-ph/0012136.

[86] N. Kivel, M. V. Polyakov, A. Schafer and O. V. Teryaev, Phys. Lett. B 497, 73 (2001), hep-ph/0007315.

[87] A. V. Belitsky, D. Muller, A. Kirchner and A. Schafer, Phys. Rev. D 64, 116002 (2001), hep-ph/0011314.

[88] A. V. Belitsky and D. Muller, Phys. Lett. B 507, 173 (2001), hep$\mathrm{ph} / 0102224$. 
[89] A. V. Belitsky, A. Kirchner, D. Muller and A. Schafer, Phys. Lett. B 510, 117 (2001), hep-ph/0103343.

[90] O. Nachtmann, Nucl. Phys. B 63, 237 (1973).

[91] H. Georgi and H. D. Politzer, Phys. Rev. D 14, 1829 (1976).

[92] P. A. M. Guichon and M. Vanderhaeghen, Prog. Part. Nucl. Phys. 41, 125 (1998), hep-ph/9806305.

[93] M. Gluck, E. Reya and A. Vogt, Z. Phys. C 67, 433 (1995).

[94] I. V. Musatov, Virtual Compton Scattering Processes in Quantum Chromodynamics, Ph.D. Thesis (1999), UMI-99-49834.

[95] A. V. Belitsky, D. Muller and A. Kirchner, Nucl. Phys. B 629, 323 (2002), hep-ph/0112108.

[96] M. Goshtasbpour and G. P. Ramsey, Phys. Rev. D 55, 1244 (1997), hep$\mathrm{ph} / 9512250$.

[97] M. Penttinen, M. V. Polyakov and K. Goeke, Phys. Rev. D 62, 014024 (2000), hep-ph/9909489.

[98] S. Stepanyan et al. [CLAS Collaboration], Phys. Rev. Lett. 87, 182002 (2001), hep-ex/0107043.

[99] A. Airapetian et al. [HERMES Collaboration], Phys. Rev. Lett. 87, 182001 (2001), hep-ex/0106068.

[100] A. Psaker, Braz. J. Phys. 34, 944 (2004), hep-ph/0404181.

[101] S. J. Brodsky, J. F. Gunion and R. L. Jaffe, Phys. Rev. D 6, 2487 (1972).

[102] J. D. Bjorken and E. A. Paschos, Phys. Rev. D 1, 1450 (1970).

[103] W. Greiner and J. Reinhardt, Quantum Electrodynamics, Berlin, Germany: Springer-Verlag (1994).

[104] E. R. Berger, M. Diehl and B. Pire, Eur. Phys. J. C 23, 675 (2002), hep$\mathrm{ph} / 0110062$. 
[105] D. Drakoulakos et al. [Minerva Collaboration], hep-ex/0405002.

[106] P. Amore, C. Coriano and M. Guzzi, JHEP 0502, 038 (2005), hep$\mathrm{ph} / 0404121$.

[107] C. Coriano and M. Guzzi, Phys. Rev. D 71, 053002 (2005), hep-ph/0411253.

[108] A. Psaker, hep-ph/0412321.

[109] A. Psaker, A. V. Radyushkin and W. Melnitchouk, Weak Deeply Virtual Compton Scattering, work in progress.

[110] L. Mankiewicz, G. Piller and T. Weigl, Phys. Rev. D 59, 017501 (1999), hep-ph/9712508.

[111] A. Psaker, A. V. Radyushkin, Double Distributions, Feynman Mechanism and RCS on the Pion, paper under preparation.

[112] A. Psaker, A. V. Radyushkin, Double Distributions and Wide-Angle Real Compton Scattering on the Nucleon, paper under preparation.

[113] A. Psaker, A. V. Radyushkin, Exclusive Production of Pions in the TwoPhoton Collisions in the Double-Distribution Approach, work in progress.

[114] V. B. Berestetskii, E. M. Lifshitz and L. P. Pitaevskii, Quantum Electrodynamics, Pergamon Press (1982).

[115] A. Mukherjee, I. V. Musatov, H. C. Pauli and A. V. Radyushkin, Phys. Rev. D 67, 073014 (2003), hep-ph/0205315. 


\section{APPENDIX A}

\section{GLOSSARY}

The following abbreviations are often used in a text:

$\begin{array}{rrl}\text { DA } & \ldots \ldots \ldots \ldots \ldots \ldots & \text { distribution amplitude } \\ \text { DD } & \ldots \ldots \ldots \ldots \ldots \ldots & \text { double distribution } \\ \text { DIS } & \ldots \ldots \ldots \ldots \ldots \ldots & \text { deeply inelastic lepton scattering } \\ \text { DMP } & \ldots \ldots \ldots \ldots \ldots \ldots & \text { deeply exclusive meson production } \\ \text { DVCS } & \ldots \ldots \ldots \ldots \ldots \ldots & \text { deeply virtual Compton scattering } \\ \text { GPD } & \ldots \ldots \ldots \ldots \ldots \ldots & \text { generalized parton distribution } \\ \text { OPE } & \ldots \ldots \ldots \ldots \ldots \ldots & \text { operator product expansion } \\ \text { PDF } & \ldots \ldots \ldots \ldots \ldots \ldots & \text { parton distribution function } \\ \text { QCD } & \ldots \ldots \ldots \ldots \ldots \ldots & \text { quantum chromodynamics } \\ \text { QED } & \ldots \ldots \ldots \ldots \ldots \ldots . & \text { quantum electrodynamics } \\ \text { TCS } & \ldots \ldots \ldots \ldots \ldots \ldots . & \text { time-like Compton scattering } \\ \text { VCA } & \ldots \ldots \ldots \ldots \ldots \ldots & \text { virtual Compton scattering amplitude } \\ \text { WACS } & \ldots \ldots \ldots \ldots \ldots \ldots & \text { wide }- \text { angle real Compton scattering }\end{array}$




\section{APPENDIX B}

\section{LIST OF INTEGRALS AND SCALAR FUNCTIONS THAT APPEAR IN THE BETHE-HEITLER SUBPROCESS OF THE INCLUSIVE PHOTOPRODUCTION OF LEPTON PAIRS}

In Section V.3, we compute the double integral (182). The calculation is carried out through the following set of integrals:

$$
\begin{aligned}
& \int d^{4} k \delta^{+}() \delta^{+}() \frac{1}{(k \cdot q)}=I_{0} \\
& \int d^{4} k \delta^{+}() \delta^{+}() \frac{k^{\mu}}{(k \cdot q)}=I_{1} q^{\mu}+I_{2} q^{\mu}, \\
& \int d^{4} k \delta^{+}() \delta^{+}() \frac{k^{\mu} k^{\rho}}{(k \cdot q)}=I_{3} q^{\mu} q^{\rho}+I_{4} q^{\prime \mu} q^{\prime \rho} \\
& +I_{5}\left(q^{\mu} q^{\rho}+q^{\rho} q^{\prime \mu}\right)+I_{6} g^{\mu \rho} \\
& \int d^{4} k \delta^{+}() \delta^{+}() \frac{1}{\left(q \cdot q^{\prime}\right)-(k \cdot q)}=J_{0} \\
& \int d^{4} k \delta^{+}() \delta^{+}() \frac{k^{\mu}}{\left(q \cdot q^{\prime}\right)-(k \cdot q)}=J_{1} q^{\mu}+J_{2} q^{\prime \mu}, \\
& \int d^{4} k \delta^{+}() \delta^{+}() \frac{k^{\mu} k^{\rho}}{\left(q \cdot q^{\prime}\right)-(k \cdot q)}=J_{3} q^{\mu} q^{\rho}+J_{4} q^{\mu} q^{\rho} \\
& +J_{5}\left(q^{\mu} q^{\rho}+q^{\rho} q^{\mu}\right)+J_{6} g^{\mu \rho} \\
& \int d^{4} k \delta^{+}() \delta^{+}() \frac{1}{(k \cdot q)\left[\left(q \cdot q^{\prime}\right)-(k \cdot q)\right]}=\frac{1}{\left(q \cdot q^{\prime}\right)}\left(I_{0}+J_{0}\right) \text {, } \\
& \int d^{4} k \delta^{+}() \delta^{+}() \frac{\left(k \cdot q^{\prime}\right)}{(k \cdot q)\left[\left(q \cdot q^{\prime}\right)-(k \cdot q)\right]}=\frac{q^{\prime 2}}{2\left(q \cdot q^{\prime}\right)}\left(I_{0}+J_{0}\right) \text {, } \\
& \int d^{4} k \delta^{+}() \delta^{+}() \frac{\left(k \cdot q^{\prime}\right)^{2}}{(k \cdot q)\left[\left(q \cdot q^{\prime}\right)-(k \cdot q)\right]}=\frac{q^{4}}{4\left(q \cdot q^{\prime}\right)}\left(I_{0}+J_{0}\right) \text {, } \\
& \int d^{4} k \delta^{+}() \delta^{+}() \frac{(k \cdot q)}{\left(q \cdot q^{\prime}\right)-(k \cdot q)}=\left(q \cdot q^{\prime}\right) J_{2} \\
& \int d^{4} k \delta^{+}() \delta^{+}() \frac{\left(k \cdot q^{\prime}\right) k^{\mu}}{(k \cdot q)\left[\left(q \cdot q^{\prime}\right)-(k \cdot q)\right]}=K_{1} q^{\mu}+K_{2} q^{\prime \mu}, \\
& \int d^{4} k \delta^{+}() \delta^{+}() \frac{\left(k \cdot q^{\prime}\right) k^{\mu} k^{\rho}}{(k \cdot q)\left[\left(q \cdot q^{\prime}\right)-(k \cdot q)\right]}=K_{3} q^{\mu} q^{\rho}+K_{4} q^{\prime \mu} q^{\prime \rho} \\
& +K_{5}\left(q^{\mu} q^{\rho}+q^{\rho} q^{\prime \mu}\right)+K_{6} g^{\mu \rho},
\end{aligned}
$$




$$
\begin{aligned}
\int d^{4} k \delta^{+}() \delta^{+}() \frac{1}{(k \cdot q)^{2}}= & L_{0} \\
\int d^{4} k \delta^{+}() \delta^{+}() \frac{k^{\mu}}{(k \cdot q)^{2}}= & L_{1} q^{\mu}+L_{2} q^{\prime \mu} \\
\int d^{4} k \delta^{+}() \delta^{+}() \frac{k^{\mu} k^{\rho}}{(k \cdot q)^{2}}= & L_{3} q^{\mu} q^{\rho}+L_{4} q^{\prime \mu} q^{\prime \rho} \\
& +L_{5}\left(q^{\mu} q^{\prime \rho}+q^{\rho} q^{\prime \mu}\right)+L_{6} g^{\mu \rho} \\
\int d^{4} k \delta^{+}() \delta^{+}() \frac{k^{\mu}}{(k \cdot q)\left[\left(q \cdot q^{\prime}\right)-(k \cdot q)\right]} & M_{1} q^{\mu}+M_{2} q^{\prime \mu} \\
\int d^{4} k \delta^{+}() \delta^{+}() \frac{k^{\mu} k^{\rho}}{(k \cdot q)\left[\left(q \cdot q^{\prime}\right)-(k \cdot q)\right]}= & M_{3} q^{\mu} q^{\rho}+M_{4} q^{\prime \mu} q^{\prime \rho} \\
& +M_{5}\left(q^{\mu} q^{\prime \rho}+q^{\rho} q^{\prime \mu}\right)+M_{6} g^{\mu \rho} .
\end{aligned}
$$

The abbreviation $\delta^{+}() \delta^{+}() \equiv \delta^{+}\left(k^{2}-m^{2}\right) \delta^{+}\left[\left(q^{\prime}-k\right)^{2}-m^{2}\right]$ is being used together with the notation $\delta^{+}\left(k^{2}-m^{2}\right) \equiv \delta\left(k^{2}-m^{2}\right) \theta\left(k_{0}\right)$, where $m$ labels the lepton mass. The coefficients on the right-hand side of Eq. (351) are scalar functions, which depend upon three invariants, i.e. $m^{2}, q^{2}$ and $\left(q \cdot q^{\prime}\right)=\left(q^{2}-t\right) / 2$, and can be derived by contracting both sides of Eq. (351) with the four-momenta $q_{\mu}$ and $q_{\mu}^{\prime}$ of the initial real and final virtual photons, respectively, and with the tensors $q_{\mu} q_{\rho}, q_{\mu}^{\prime} q_{\rho}^{\prime}, q_{\mu}^{\prime} q_{\rho}$ and $g_{\mu \rho}$. These functions can be further reduced to the expressions written in terms of only two scalar functions, i.e. $I_{0}$ and $I_{2}$. After some algebra we obtain

$$
\begin{aligned}
& I_{0}=\frac{\pi}{2\left(q \cdot q^{\prime}\right)} \ln \left[\frac{1+\sqrt{1-4 m^{2} / q^{\prime 2}}}{1-\sqrt{1-4 m^{2} / q^{\prime 2}}}\right] \\
& I_{1}=\frac{q^{\prime 2}}{2\left(q \cdot q^{\prime}\right)}\left[I_{0}-2 I_{2}\right] \\
& I_{2}=\frac{\pi}{2\left(q \cdot q^{\prime}\right)} \sqrt{1-\frac{4 m^{2}}{q^{\prime 2}}} \\
& I_{3}=\frac{q^{\prime 2}}{2\left(q \cdot q^{\prime}\right)^{2}}\left[\left(\frac{q^{2}}{2}+m^{2}\right) I_{0}-\frac{3 q^{2}}{2} I_{2}\right] \\
& I_{4}=\frac{1}{2} I_{2}, \\
& I_{5}=-\frac{1}{2\left(q \cdot q^{\prime}\right)}\left[m^{2} I_{0}-\frac{q^{2}}{2} I_{2}\right] \\
& I_{6}=\frac{1}{2}\left[m^{2} I_{0}-\frac{q^{2}}{2} I_{2}\right] \\
& J_{0}=I_{0},
\end{aligned}
$$




$$
\begin{aligned}
& J_{1}=-\frac{q^{2}}{2\left(q \cdot q^{\prime}\right)}\left[I_{0}-2 I_{2}\right], \\
& J_{2}=I_{0}-I_{2} \text {, } \\
& J_{3}=\frac{q^{\prime 2}}{2\left(q \cdot q^{\prime}\right)^{2}}\left[\left(\frac{q^{\prime 2}}{2}+m^{2}\right) I_{0}-\frac{3 q^{2}}{2} I_{2}\right], \\
& J_{4}=I_{0}-\frac{3}{2} I_{2}, \\
& J_{5}=-\frac{1}{2\left(q \cdot q^{\prime}\right)}\left[\left(q^{\prime 2}+m^{2}\right) I_{0}-\frac{5 q^{\prime 2}}{2} I_{2}\right] \text {, } \\
& J_{6}=\frac{1}{2}\left[m^{2} I_{0}-\frac{q^{\prime 2}}{2} I_{2}\right] \text {, } \\
& K_{1}=0 \text {, } \\
& K_{2}=\frac{q^{2}}{2\left(q \cdot q^{\prime}\right)} I_{0}, \\
& K_{3}=\frac{q^{\prime 4}}{2\left(q \cdot q^{\prime}\right)^{3}}\left[\left(\frac{q^{\prime 2}}{2}+m^{2}\right) I_{0}-\frac{3 q^{\prime 2}}{2} I_{2}\right], \\
& K_{4}=\frac{q^{\prime 2}}{2\left(q \cdot q^{\prime}\right)}\left[I_{0}-I_{2}\right] \text {, } \\
& K_{5}=-\frac{q^{\prime 2}}{2\left(q \cdot q^{\prime}\right)^{2}}\left[\left(\frac{q^{\prime 2}}{2}+m^{2}\right) I_{0}-\frac{3 q^{\prime 2}}{2} I_{2}\right] \text {, } \\
& K_{6}=\frac{q^{\prime 2}}{2\left(q \cdot q^{\prime}\right)}\left[m^{2} I_{0}-\frac{q^{\prime 2}}{2} I_{2}\right], \\
& L_{0}=\frac{q^{2}}{m^{2}\left(q \cdot q^{\prime}\right)} I_{2} \\
& L_{1}=-\frac{q^{\prime 2}}{\left(q \cdot q^{\prime}\right)^{2}}\left[I_{0}-\frac{q^{2}}{2 m^{2}} I_{2}\right] \text {, } \\
& L_{2}=\frac{1}{\left(q \cdot q^{\prime}\right)} I_{0}, \\
& L_{3}=-\frac{q^{4}}{4\left(q \cdot q^{\prime}\right)^{3}}\left[6 I_{0}-\left(\frac{q^{2}}{m^{2}}+8\right) I_{2}\right], \\
& L_{4}=\frac{1}{\left(q \cdot q^{\prime}\right)} I_{2}, \\
& L_{5}=\frac{q^{2}}{\left(q \cdot q^{\prime}\right)^{2}}\left[I_{0}-2 I_{2}\right] \text {, } \\
& L_{6}=-\frac{q^{2}}{2\left(q \cdot q^{\prime}\right)}\left[I_{0}-2 I_{2}\right], \\
& M_{1}=0 \text {, } \\
& M_{2}=\frac{1}{\left(q \cdot q^{\prime}\right)} I_{0},
\end{aligned}
$$




$$
\begin{aligned}
& M_{3}=\frac{q^{\prime 2}}{\left(q \cdot q^{\prime}\right)^{3}}\left[\left(\frac{q^{2}}{2}+m^{2}\right) I_{0}-\frac{3 q^{2}}{2} I_{2}\right], \\
& M_{4}=\frac{1}{\left(q \cdot q^{\prime}\right)}\left[I_{0}-I_{2}\right] \\
& M_{5}=-\frac{1}{\left(q \cdot q^{\prime}\right)^{2}}\left[\left(\frac{q^{2}}{2}+m^{2}\right) I_{0}-\frac{3 q^{2}}{2} I_{2}\right], \\
& M_{6}=\frac{1}{\left(q \cdot q^{\prime}\right)}\left[m^{2} I_{0}-\frac{q^{2}}{2} I_{2}\right] .
\end{aligned}
$$




\section{APPENDIX C}

\section{MODIFIED PARAMETRIZATION OF THE NONFORWARD MATRIX ELEMENT IN THE SCALAR TOY MODEL}

We modify the scalar parametrization $\mathcal{F}(z, p, r)$ of the nonforward matrix element $\langle p-r / 2|\phi(-z / 2) \phi(z / 2)| p+r / 2\rangle$, introduced in Section VIII.2, by adding the subleading $\mathcal{O}\left(k^{2} z^{2}\right)$ and $\mathcal{O}\left(k^{4} z^{4}\right)$ terms. Namely,

$$
\begin{array}{r}
\langle p-r / 2|\phi(-z / 2) \phi(z / 2)| p+r / 2\rangle=\int_{-1}^{1} d \beta \int_{-1+|\beta|}^{1-|\beta|} d \alpha e^{i(k \cdot z)} \\
\times\left[h_{f}(\beta, \alpha, t)+\frac{k^{2} z^{2}}{4} h_{2 f}(\beta, \alpha, t)+\frac{k^{4} z^{4}}{32} h_{4 f}(\beta, \alpha, t)\right],
\end{array}
$$

where $k=\beta p+\alpha r / 2$. The function $h_{f}(\beta, \alpha, t)$ is the original double distribution or the so-called parent DD. The new functions, known as the daughter DDs, $h_{2 f}(\beta, \alpha, t)$ and $h_{4 f}(\beta, \alpha, t)$, are not independent but rather to be determined in such a way that the matrix element $\langle p-r / 2|\phi(-z / 2) \phi(z / 2)| p+r / 2\rangle$ has only terms of the certain twist. In particular, the twist-2 part of the matrix element should satisfy the d'Alembert equation with respect to $z$,

$$
\partial^{2}\langle p-r / 2|\phi(-z / 2) \phi(z / 2)| p+r / 2\rangle_{t w i s t-2}=0 .
$$

By imposing this condition on the right-hand side of Eq. (353), one can determine both $h_{2 f}(\beta, \alpha, t)$ and $h_{4 f}(\beta, \alpha, t)$. Thus in the lowest order in $z^{2}$ we find

$$
\begin{gathered}
\int_{-1}^{1} d \beta \int_{-1+|\beta|}^{1-|\beta|} d \alpha e^{i(k \cdot z)} k^{2} \\
\times\left[-h_{f}(\beta, \alpha, t)+i(k \cdot z) h_{2 f}(\beta, \alpha, t)+2 h_{2 f}(\beta, \alpha, t)\right]=0 .
\end{gathered}
$$

The second term in the brackets can be written as

$$
\int_{-1}^{1} d \beta \int_{-1+|\beta|}^{1-|\beta|} d \alpha h_{2 f}(\beta, \alpha, t) k^{2}\left(\beta \frac{\partial}{\partial \beta}+\alpha \frac{\partial}{\partial \alpha}\right) e^{i(k \cdot z)},
$$

and further integrating it by parts and using the boundary conditions for $h_{2 f}(\beta, \alpha, t)$ (note that, like the parent DD, both daughter distributions vanish at the boundaries of the support region), it takes the form

$$
-\int_{-1}^{1} d \beta \int_{-1+|\beta|}^{1-|\beta|} d \alpha e^{i(k \cdot z)} k^{2}\left(\beta \frac{\partial}{\partial \beta}+\alpha \frac{\partial}{\partial \alpha}+2\right) h_{2 f}(\beta, \alpha, t) .
$$


Substituting Eq. (357) back into Eq (355) leads to the partial differential equation for $h_{2 f}(\beta, \alpha, t)$,

$$
\left(\beta \frac{\partial}{\partial \beta}+\alpha \frac{\partial}{\partial \alpha}\right) h_{2 f}(\beta, \alpha, t)=-h_{f}(\beta, \alpha, t)
$$

with the solution

$$
h_{2 f}(\beta, \alpha, t)=\int_{1}^{\infty} d \tau \tau h_{f}(\tau \beta, \tau \alpha, t) .
$$

It is easy to see that this solution indeed satisfies Eq. (358) since

$$
\begin{aligned}
\int_{1}^{\infty} d \tau \tau\left(\beta \frac{\partial}{\partial \beta}+\alpha \frac{\partial}{\partial \alpha}\right) h_{f}(\tau \beta, \tau \alpha, t)= & \int_{1}^{\infty} d \tau \tau^{2} \frac{\partial}{\partial \tau} h_{f}(\tau \beta, \tau \alpha, t) \\
= & -h_{f}(\beta, \alpha, t) \\
& -2 \int_{1}^{\infty} d \tau \tau h_{f}(\tau \beta, \tau \alpha, t) .
\end{aligned}
$$

Similarly, in the next order in the $z^{2}$ expansion, the harmonic condition (354) gives the partial differential equation for the daughter $\operatorname{DD} h_{4 f}(\beta, \alpha, t)$,

$$
\left(\beta \frac{\partial}{\partial \beta}+\alpha \frac{\partial}{\partial \alpha}-1\right) h_{4 f}(\beta, \alpha, t)=-h_{2 f}(\beta, \alpha, t) .
$$

The solution is

$$
h_{4 f}(\beta, \alpha, t)=\int_{1}^{\infty} d \tau h_{2 f}(\tau \beta, \tau \alpha, t) .
$$

It is worth noting at this point that to evaluate the twist-2 and twist-3 contributions to the Compton scattering amplitude using a modified parametrization (353), the inclusion of $z^{2}$ and $z^{4}$ terms requires, in addition to Eqs. (98) and (317), four more integrals over $z$. Here we present the list of all six integrals

$$
\begin{aligned}
\int d^{4} z e^{i(l \cdot z)} \frac{z_{\rho}}{2 \pi^{2}\left(z^{2}-i 0\right)} & =\frac{4 l_{\rho}}{\left(l^{2}+i 0\right)^{2}} \\
\int d^{4} z e^{i(l \cdot z)} \frac{z_{\rho}}{2 \pi^{2}\left(z^{2}-i 0\right)^{2}} & =\frac{l_{\rho}}{l^{2}+i 0}, \\
\int d^{4} z e^{i(l \cdot z)} \frac{i z_{\rho} z_{\sigma}}{2 \pi^{2}\left(z^{2}-i 0\right)} & =4 \frac{g_{\rho \sigma} l^{4}-4 l_{\rho} l_{\sigma} l^{2}}{\left(l^{2}+i 0\right)^{4}} \\
\int d^{4} z e^{i(l \cdot z)} \frac{i z_{\rho} z_{\sigma}}{2 \pi^{2}\left(z^{2}-i 0\right)^{2}} & =\frac{g_{\rho \sigma} l^{2}-2 l_{\rho} l_{\sigma}}{\left(l^{2}+i 0\right)^{2}} \\
\int d^{4} z e^{i(l \cdot z)} \frac{z_{\delta} z_{\rho} z_{\lambda}}{2 \pi^{2}\left(z^{2}-i 0\right)} & =16 \frac{\left(g_{\delta \rho} l_{\lambda}+g_{\delta \lambda} l_{\rho}+g_{\rho \lambda} l_{\delta}\right) l^{2}-l_{\delta} l_{\rho} l_{\lambda}}{\left(l^{2}+i 0\right)^{4}} \\
\int d^{4} z e^{i(l \cdot z)} \frac{z_{\delta} z_{\rho} z_{\lambda}}{2 \pi^{2}\left(z^{2}-i 0\right)^{2}} & =2 \frac{\left(g_{\delta \rho} l_{\lambda}+g_{\delta \lambda} l_{\rho}+g_{\rho \lambda} l_{\delta}\right) l^{4}-4 l_{\delta} l_{\rho} l_{\lambda} l^{2}}{\left(l^{2}+i 0\right)^{4}} .
\end{aligned}
$$




\title{
VITA
}

\author{
A. Psaker \\ Department of Physics \\ Old Dominion University \\ Norfolk, VA 23529
}

B.S. in Physics, University of Ljubljana, March 1997

M.S. in Physics, Old Dominion University, May 2001

Typeset using LATEX. $_{\mathrm{E}} \mathrm{X}$. 